\title{
Sq and EEJ-A Review on the Daily Variation of the Geomagnetic Field Caused by Ionospheric Dynamo Currents
}

\author{
Y. Yamazaki ${ }^{1}$ (D) A. Maute ${ }^{2}$
}

Received: 8 April 2016/ Accepted: 23 August 2016 / Published online: 14 September 2016

(c) The Author(s) 2016. This article is published with open access at Springerlink.com

\begin{abstract}
A record of the geomagnetic field on the ground sometimes shows smooth daily variations on the order of a few tens of nano teslas. These daily variations, commonly known as $\mathrm{Sq}$, are caused by electric currents of several $\mu \mathrm{A} / \mathrm{m}^{2}$ flowing on the sunlit side of the Eregion ionosphere at about $90-150 \mathrm{~km}$ heights. We review advances in our understanding of the geomagnetic daily variation and its source ionospheric currents during the past 75 years. Observations and existing theories are first outlined as background knowledge for the nonspecialist. Data analysis methods, such as spherical harmonic analysis, are then described in detail. Various aspects of the geomagnetic daily variation are discussed and interpreted using these results. Finally, remaining issues are highlighted to provide possible directions for future work.
\end{abstract}

Keywords Geomagnetic field · Solar quiet · Daily variation · Ionospheric currents · Sq · EEJ

\section{Introduction}

\subsection{Scope of This Review}

The purpose of this review is to provide an overview of the geomagnetic daily variation and its primary source currents in the E-region ionosphere $(90-150 \mathrm{~km})$, mainly from the perspective of surface geomagnetic field measurements. We focus on accomplishments since 1940 when Chapman and Bartels published two volumes of the book "Geomagnetism", which includes a comprehensive overview of earlier works on the geomagnetic daily variation. Since Chapman and Bartels (1940), a number of review articles have been published on this topic (see Table 1). The present paper attempts to integrate those knowledge and also

\section{Y. Yamazaki}

y.yamazaki@lancaster.ac.uk

1 Department of Physics, University of Lancaster, Lancaster LA1 4YB, UK

2 High Altitude Observatory, National Center for Atmospheric Research, Boulder, CO 80307-3000, USA 
Table 1 Review articles on $\mathrm{Sq}$ and/or EEJ

${ }^{1}$ History

2 Ground magnetic data

3 Radar

4 Rocket

5 Numerical models

${ }^{6}$ Data analysis technique

\begin{tabular}{|c|c|}
\hline Source & Focused topics \\
\hline Matsushita (1967) & Sq, L, dynamo theory $1,2,4,5,6$ \\
\hline Matsushita (1968) & $\mathrm{Sq}, \mathrm{L}^{1,2}$ \\
\hline Richmond (1979) & Dynamo theory ${ }^{1,5}$ \\
\hline Wagner et al. (1980) & Sq, dynamo theory 2,5 \\
\hline Richmond (1989) & Dynamo theory ${ }^{5}$ \\
\hline Forbes (1981) & Equatorial electrojet ${ }^{1,2,3,4,5}$ \\
\hline Campbell (1989a) & Sq, upper mantle conductivity $1,2,6$ \\
\hline Campbell (1989b) & $\mathrm{Sq}^{1,2}$ \\
\hline Rastogi (1989) & Equatorial electrojet ${ }^{2,3,4,5}$ \\
\hline Reddy (1989) & Equatorial electrojet ${ }^{1,2,3,4}$ \\
\hline Richmond (1995a) & Sq, equatorial electrojet, dynamo theory $2,3,4,5$ \\
\hline Richmond (1995b) & Dynamo theory ${ }^{1,5}$ \\
\hline Onwumechili (1998) & Sq, equatorial electrojet ${ }^{1,2,3,4,5}$ \\
\hline Campbell (2003) & $\mathrm{Sq}^{1,2,6}$ \\
\hline
\end{tabular}

highlight recent progress. Although the establishment of knowledge has been made through various types of observations (e.g., ground measurements, rockets, aircrafts, and satellites) along with theoretical studies, the scope of this review is limited to the aspects related to ground-based magnetometer data. We will discuss "what needs to be known to understand the data", "how to analyze the data", and "what can be learned from the data". The results from other types of data, such as from satellites, are referred to only when they are essential for scientific interpretations outlined in this review. Also, high-latitude processes are not detailed as they involve different physical mechanisms than at low and middle latitudes. These restrictions were necessary to keep the size of the review manageable. For the topics that are not covered in this review, readers are referred to the literature in Table 1 and references therein. So far there is no review on the satellite observations of the global Sq field. Thus, interested readers are referred to recent articles by Stolle et al. (2016) and Chulliat et al. (2016). Finally, the scope of this review is not limited to geomagnetically quiet periods. We will address how the E-region currents respond to forcing from various sources (e.g., the Sun, magnetosphere, and lower atmosphere) and how that affects the geomagnetic daily variations at middle and low latitudes.

\subsection{Observational Overview}

A record of the Earth's magnetic field represents a superposition of signals from various sources. By far the most significant contribution comes from electric currents generated in the Earth's core that produce the magnetic field of several tens of thousands of nano teslas on the surface, the so-called main field. All other sources account for only a fraction of the observed geomagnetic field (up to a few percent). Among them are electric currents flowing in the ionosphere. Ionospheric currents are responsible for the regular daily variation of the geomagnetic field on the order of a few tens of nano teslas, which is often referred to as solar quiet $(\mathrm{Sq})$. Sq is a "solar" variation because it depends primarily on solar (local) time. Also, Sq is a "quiet" variation because it is visible only when solar-wind driven disturbances are absent. Geomagnetic disturbances associated with storms and substorms are typically several hundreds of nano teslas on the surface, 
Fig. 1 (Top) The horizontal $(H)$ component geomagnetic field at Hermanus $\left(34.4^{\circ} \mathrm{S}, 19.2^{\circ} \mathrm{E}\right)$, South Africa in November 2001. (Bottom) The interplanetary magnetic field, $\mathrm{Bt}$ and $\mathrm{Bz}$ components.
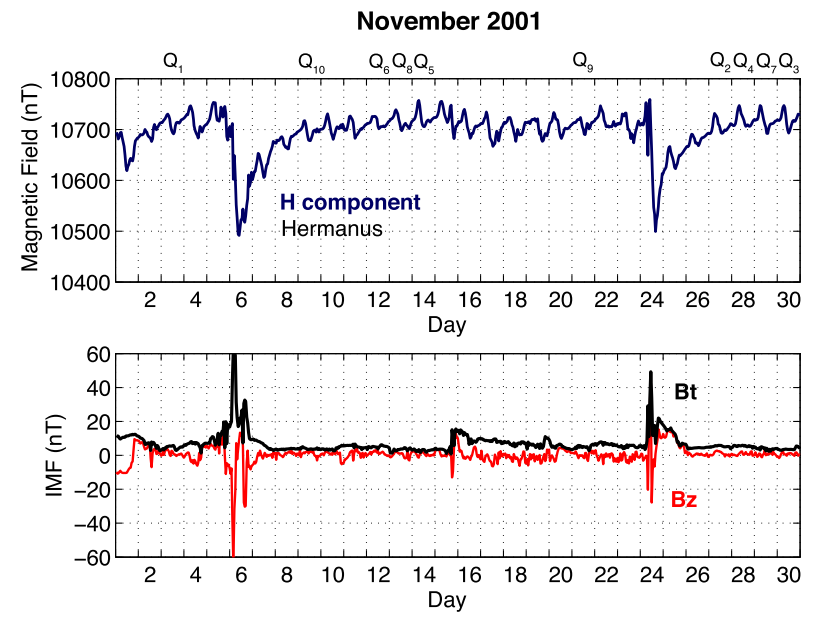

which can easily mask underlying Sq signals. Despite the small amplitude, studies on Sq have been important for understanding the ionospheric electrodynamics (Richmond 1979, 1995b) and its coupling to the magnetosphere and lower atmosphere (Wagner et al. 1980; Richmond 1995b); for determining a base level for geomagnetic indices (Mayaud 1980; Love and Gannon 2009; Gjerloev 2012); for monitoring solar radiation activity (Svalgaard and Cliver 2007; Svalgaard 2016); and for estimating electrical conductivity within the Earth (Campbell and Schiffmacher 1988a; Campbell et al. 1998; Okeke and Obiora 2016).

The upper panel of Fig. 1 shows a monthly record of the horizontal $H$ component $^{1}$ of the geomagnetic field observed at Hermanus $\left(34.4^{\circ} \mathrm{S}\right.$ geographic latitude, $19.2^{\circ} \mathrm{E}$ geographic longitude), South Africa in November 2001. The main core field accounts for most of the average field of $\sim 10,700 \mathrm{nT}$. The temporal variation of the geomagnetic field is mostly due to external contributions (i.e., magnetospheric and ionospheric currents). The depression in the $H$-component geomagnetic field by $\sim 200 \mathrm{nT}$ during 6-8 November and 24-26 November indicates the occurrence of geomagnetic storms. From the bottom panel of Fig. 1, which shows the interplanetary magnetic field (IMF), it is obvious that those geomagnetic storms are caused by solar-wind disturbances. During a geomagnetic storm, a westward ring current develops in the magnetosphere, which leads to a global-scale reduction in the $H$-component geomagnetic field (see a review by Kamide and Maltsev 2007). In Fig. 1, $\mathrm{Q}_{1}-\mathrm{Q}_{10}$ indicates International Quiet Days (IQDs), which denote geomagnetically quietest days of the month. The IQDs are routinely selected for each month and published by Deutsches GeoForschungsZentrum (GFZ German Research Centre for Geosciences). It can be seen that IQDs coincide with periods when the IMF is relatively small. When solar-wind disturbances are absent, the geomagnetic field exhibits rather smooth and regular daily changes, which represent $\mathrm{Sq}$ variations.

In the book, Geomagnetism, Chapman and Bartels (1940, p. 214) defined Sq as the "average" quiet daily variation over five quietest days of a month (i.e., $\mathrm{Q}_{1}-\mathrm{Q}_{5}$ ). Later, Mayaud (1965) proposed to call the geomagnetic daily variation on an individual day solar regu-

\footnotetext{
${ }^{1}$ Following the standard nomenclature (Jankowski and Sucksdorff 1996), elements of the geomagnetic field are denoted as follows: $H=$ horizontal component, $D=$ magnetic declination, $Z=$ vertical component (positive downward), $X=$ northward component, $Y=$ eastward component. In addition, $N$ and $E$ are used to denote the components in the local magnetic northward and eastward directions, respectively (e.g., Gjerloev 2012).
} 
$\operatorname{lar}\left(\mathrm{S}_{R}\right)$ and distinguish it from the average daily variation Sq. Although the distinction between $\mathrm{Sq}$ and $\mathrm{S}_{R}$ is still occasionally used in the literature, we do not follow this convention. Rather, we use the term Sq to address both average and individual quiet-day daily geomagnetic variations. This does not cause any confusion because the physical meaning of $\mathrm{Sq}$ and $\mathrm{S}_{R}$ is the same, i.e, the magnetic effect primarily caused by ionospheric wind dynamo currents. Also, ambiguity can be easily avoided by specifying the time interval considered, e.g., "Sq variation on 31 May 1986" and "average Sq variation for January 2012". Olsen (1997a) pointed out that there are two types of classification of geomagnetic Sq variations. One is a "descriptive" (or "statistical") classification and the other is "physical" (or "causal") classification. The former emphasizes "how to derive Sq", while the latter emphasizes "what Sq represents". In the descriptive classification, it is important to derive Sq in a certain procedure. Chapman and Bartels's approach is a typical descriptive classification. Meanwhile, in the physical classification, it is important that Sq represents a certain physical process no matter how it is derived. The approach we use in this paper is a physical classification.

The discovery of Sq goes back to the early 18th century. It was made by George Graham based on careful observations of a long magnetic needle (Graham 1724a, 1724b). Early development of Sq studies is summarized in the literature by Matsushita (1968) and Campbell (1989b), and is only briefly described herein. Among early studies, Stewart (1882) and Schuster $(1889,1908)$ made particularly important contributions to the understanding of Sq. Stewart (1882) proposed the hypothesis that geomagnetic Sq variations are caused by electric currents flowing in the electrically conducting region of the upper atmosphere. According to his dynamo theory, the motion of electrically conducting air $(\mathbf{U})$ across the Earth's magnetic field $(\mathbf{B})$ gives rise to electromotive forces $(\mathbf{U} \times \mathbf{B})$ that generate electric fields and currents. This is considered the first scientific indication of the ionosphere, although the ionosphere was officially "discovered" in the later radio-wave studies in the mid 1920's (Appleton and Barnett 1925; Breit and Tuve 1925). Schuster (1889) presented evidence that the main source of geomagnetic Sq variations is external to the Earth, that is, electric currents flow above the surface in the atmosphere. Later, he estimated the conductivity of the atmosphere that is required to explain Sq variations and attributed this conductivity to the ionization of the atmosphere caused by the solar radiation (Schuster 1908). The existence of the ionospheric currents was later confirmed experimentally by sounding rockets (e.g., Singer et al. 1951; Cahill 1959; Davis et al. 1967; Maynard 1967; Yabuzaki and Ogawa 1974; Pfaff et al. 1997). It is now well established that electric currents of the order of several $\mu \mathrm{A} / \mathrm{m}^{2}$ flow on the dayside of the E-region ionosphere, in the altitude range of $90-150 \mathrm{~km}$. This region is often referred to as the dynamo region.

The Sq variations measured on the Earth's surface contain signals not only from ionospheric currents but also from secondary currents induced in the Earth's interior. Electromagnetic variations associated with the ionospheric currents have characteristic time scales from a few hours to a day. Those time-varying fields penetrate into the region called upper mantle (about 100-600 km below the surface) and induce electric currents therein (e.g., Schmucker 1970; Campbell 1987). The strength of the induced currents is roughly one third that of the ionospheric currents (e.g., Matsushita and Maeda 1965a, 1965b). The induced currents tend to be in the opposite direction to the source ionospheric currents, and thus they act to reduce Sq variations in the vertical component and increase Sq variations in the horizontal component on the ground. In addition to the effect of electromagnetic induction in the upper mantle, the presence of the conducting ocean has a measurable impact on Sq variations, especially near the coast (e.g., Kuvshinov et al. 1999, 2007; Kuvshinov and Utada 2010). 


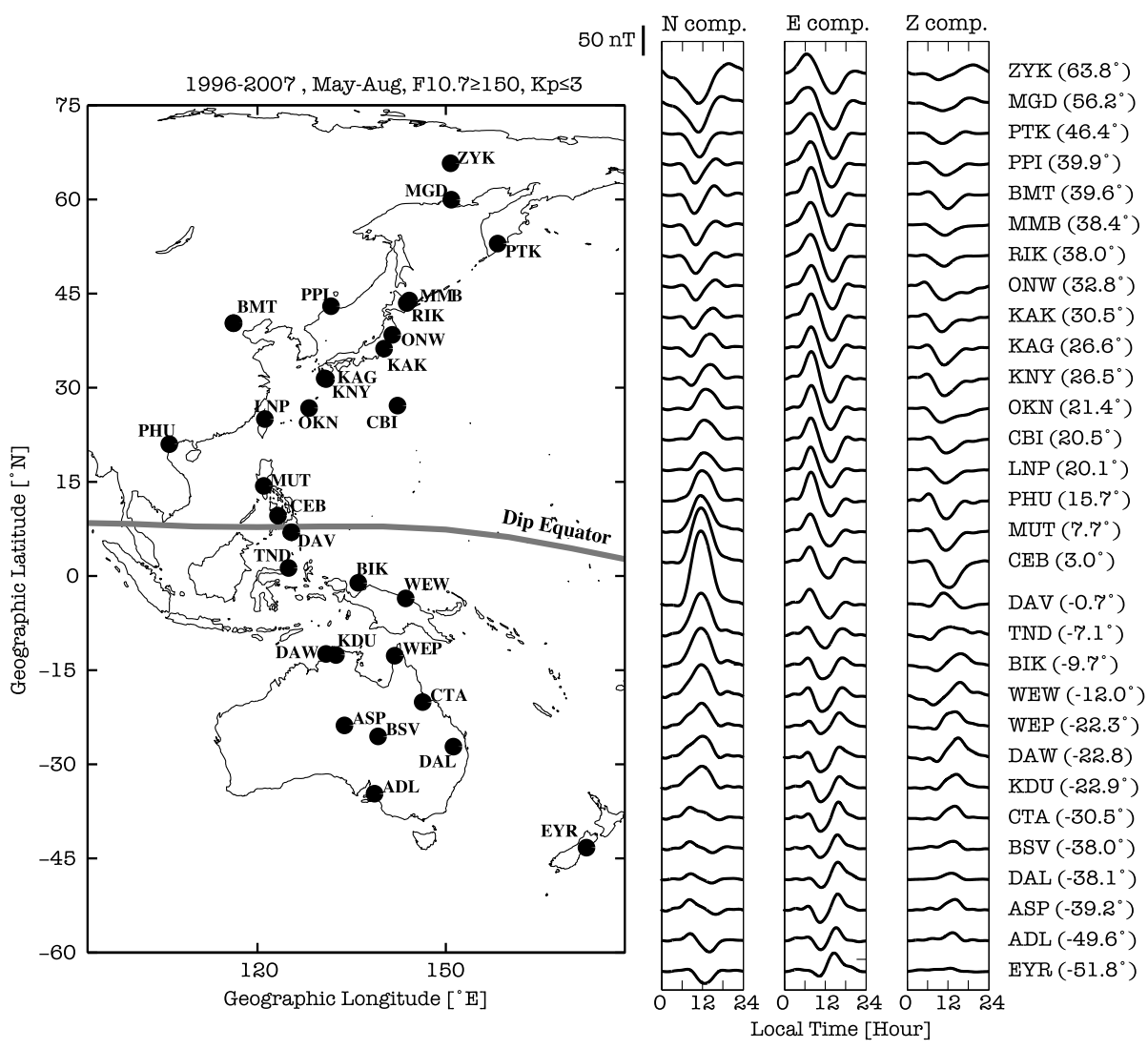

Fig. 2 Average geomagnetic daily variations in the magnetic-northward $(N)$, magnetic-eastward $(E)$, and vertically downward (Z) components during May-August of 1996-2007. From Yamazaki (2011)

Studies have shown that the Sq variations on the ground could be contaminated by the effect of various magnetospheric currents (Mead 1964; Olson 1970; Xu 1992; Olsen 1996). Especially, tail currents are believed to produce an Sq-like diurnal pattern in the geomagnetic field. According to the CHAOS-5 geomagnetic field model (Finlay et al. 2015), tail currents can cause a magnetic perturbation of $\pm 5 \mathrm{nT}$ on the Earth's surface at middle latitudes (see Lühr et al. 2016).

Geomagnetic $\mathrm{Sq}$ variations can be observed at any location on the globe. The pattern of the $\mathrm{Sq}$ variation systematically changes with latitude. Figure 2 shows the Sq variations observed at various latitudes in the East Asian sector. It can be seen that the pattern of Sq in the magnetic-northward $(N)$ component tends to be symmetric about the magnetic equator, where the geomagnetic field is completely horizontal. Meanwhile, the Sq patterns in the magnetic-eastward $(E)$ and vertical $(Z)$ components reverse at the magnetic equator. Another important feature of the latitudinal distribution of Sq is the amplification in the $N$ component near the magnetic equator. Typically, the $\mathrm{Sq}$ amplitude in $N$ at a station within $\pm 3^{\circ}$ from the magnetic equator is 2-3 times as large as that at a low-latitude station of the same longitude (say 6-9 degrees away from the magnetic equator). The equatorial enhancement of Sq can be observed in the horizontal $(H)$ and northward $(X)$ components, as well. In Fig. 2, the Sq amplitude exceeds $100 \mathrm{nT}$ in the $N$ component at Davao (DAV, 
$7.0^{\circ} \mathrm{N}, 125.4^{\circ} \mathrm{E}$ ), which is located at $0.7^{\circ}$ south of the magnetic equator. The Sq amplitude is about half at Tondano (TND), which is less than $7^{\circ}$ away from DAV. The large-amplitude Sq variation near the magnetic equator is owing to a belt of strong zonal current, which is known as the equatorial electrojet (EEJ) (Chapman 1951). A number of review articles exist about the equatorial electrojet (see Table 1), and the interested reader is referred to those reviews for early development of the topic. Satellite measurements have revealed that the center of the equatorial electrojet exists right above the magnetic equator (Lühr et al. 2004). The effective zonal ionospheric conductivity, the so-called Cowling conductivity, is locally enhanced along the magnetic equator, which gives rise to the strong zonal current of the equatorial electrojet. The driving mechanisms for $\mathrm{Sq}$ and equatorial electrojet will be discussed in Sect. 2. There is a long-standing debate as to whether the equatorial electrojet should be considered as a part of the global Sq current system or a separate current system. The Sq-EEJ relationship will be addressed in Sect. 2.5.

Although Sq variations are known to be produced by ionospheric currents, it is not possible to uniquely determine the ionospheric current system by analyzing ground Sq data only. This is because there are more than one configuration of three-dimensional (3-D) current systems that produce exactly the same Sq field on the ground; thus, one cannot tell which 3-D current system is responsible for the observed Sq variations. In order to deal with this ambiguity, it is convenient to introduce the concept of "equivalent" Sq current system. An equivalent Sq current system is a two-dimensional current system which flows in a spherical thin shell (typically assumed at $110 \mathrm{~km}$ ) and produces the same $\mathrm{Sq}$ variations as observed on the ground. The thin shell assumption of the Sq current system is reasonable because the horizontal scale of the Sq current system is much greater than the vertical scale. The horizontal extent of the Sq current system is about the size of the dayside Earth, which is approximately $\pi R_{E}\left(\sim 20,000 \mathrm{~km} ; R_{E}\right.$ is the Earth's radius), while the vertical extent of the current system is about $100 \mathrm{~km}$. The equivalent $\mathrm{Sq}$ current system can be uniquely determined from Sq data using a technique called spherical harmonic analysis, which is described in Sect. 3.7. Figure 3 displays equivalent Sq current systems at 0100 UT in different months of the year 1964 (after Takeda 1999). A current of $20 \mathrm{kA}$ flows between adjacent contour lines. The current flows in the counter-clockwise direction around positive peaks and clockwise direction around negative peaks. Despite an obvious seasonal modulation, the basic pattern of the equivalent $\mathrm{Sq}$ current system is consistent throughout the year. That is, a large-scale counter-clockwise vortex exists on the dayside of the Northern Hemisphere, and a large-scale clockwise vortex exists on the dayside of the Southern Hemisphere.

Figure 3 does not show the current systems that exist in the polar region of both the Northern and Southern Hemispheres. Figure 4 depicts a polar view of the equivalent current systems in the Northern Hemisphere during the IGY/IGC period (from July 1957 through December 1959) (after Matsushita and Xu 1982). A two-cell current pattern is visible within the polar cap (above $60^{\circ}$ magnetic latitude). A similar current system can be found in the Southern Hemisphere, but the polarity is the opposite (i.e., the current cell on the morning side is positive and the current cell on the evening side is negative.) Those polar-region current systems are driven by high-latitude electric fields generated by the solar wind-magnetospheric dynamo. During quiet periods, the current systems are mostly confined to the polar region and referred to as $\mathrm{Sq}^{P}$ (Nagata and Kokubun 1962; $\mathrm{Xu}$ 1989). During disturbed periods, the current intensity is enhanced and the current system expands to lower latitudes. The magnetic effects can be detected even in the equatorial electrojet, often showing characteristic quasi-periodic fluctuations (periods of 30-40 min) called DP2 (Nishida 1968; Kikuchi et al. 1996). 

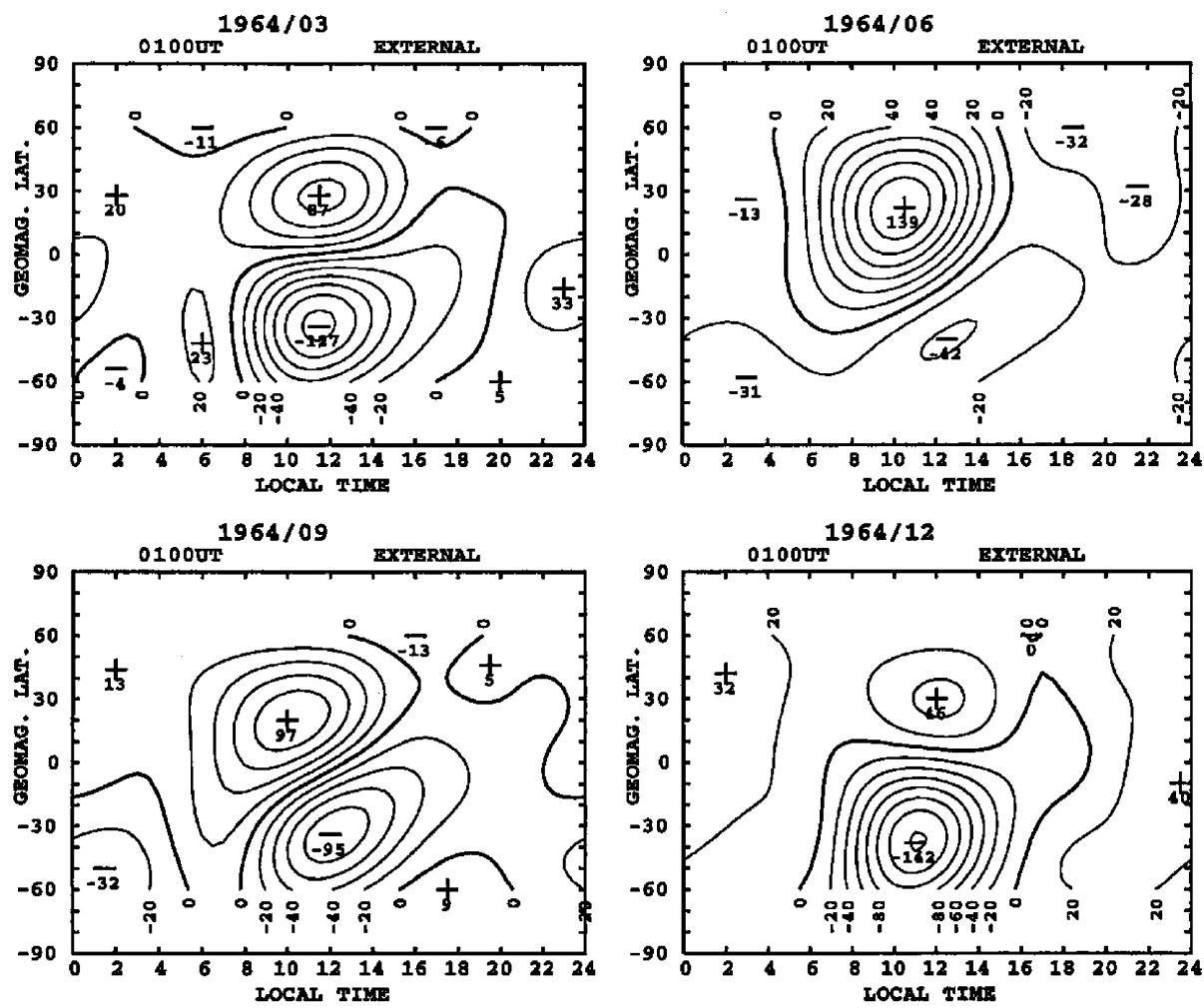

Fig. 3 Average external equivalent Sq current systems at 0100 UT in March, June, September, and December 1964. The contour intervals are $20 \mathrm{kA}$. Extreme values are indicated in kiloamperes. From Takeda (1999)

\subsection{Geomagnetic daily variations and atmospheric tides}

There is an interesting connection between the development of understanding of Sq and atmospheric tides. Atmospheric tides are global-scale oscillations of the neutral atmosphere with harmonic periods of a day, i.e., $24 \mathrm{hrs}, 12 \mathrm{hrs}, 8 \mathrm{hrs}, 6 \mathrm{hrs}$, and so on (Lindzen and Chapman 1969). Atmospheric tides can be found in various parameters such as pressure, wind, temperature, density, etc. By the 1950s, it was well known that the daily variation of the surface air pressure is dominated by the semidiurnal (12-hr) component. The large semidiurnal tide in the surface pressure was initially believed to be due to an atmospheric resonance, the period of which was predicted to be near $12 \mathrm{hrs}$ by early models (Pekeris 1937; Weekes and Wilkes 1947). Therefore, the semidiurnal tide was also thought to dominate the upper atmosphere where Sq currents are produced. However, Maeda $(1955,1957)$ and Kato (1956, 1957), estimating upper atmospheric winds from geomagnetic Sq variations, found that the primary driver for Sq currents is not the semidiurnal tide but diurnal (24-hr) tide, thus casting doubt on the resonance hypothesis. Later, the resonance theory was proven to be ineffective, as rocket measurements revealed that the peak temperature of the mesosphere is lower than that required by the resonance hypothesis. Kato (1966) recognized the importance of the "negative-mode" diurnal tide to explain the latitudinal distribution of Sq. Negative-mode tides are evanescent in the vertical direction and thus the waves are trapped at the height of the source. The negative-mode diurnal tide is generated in the thermosphere 

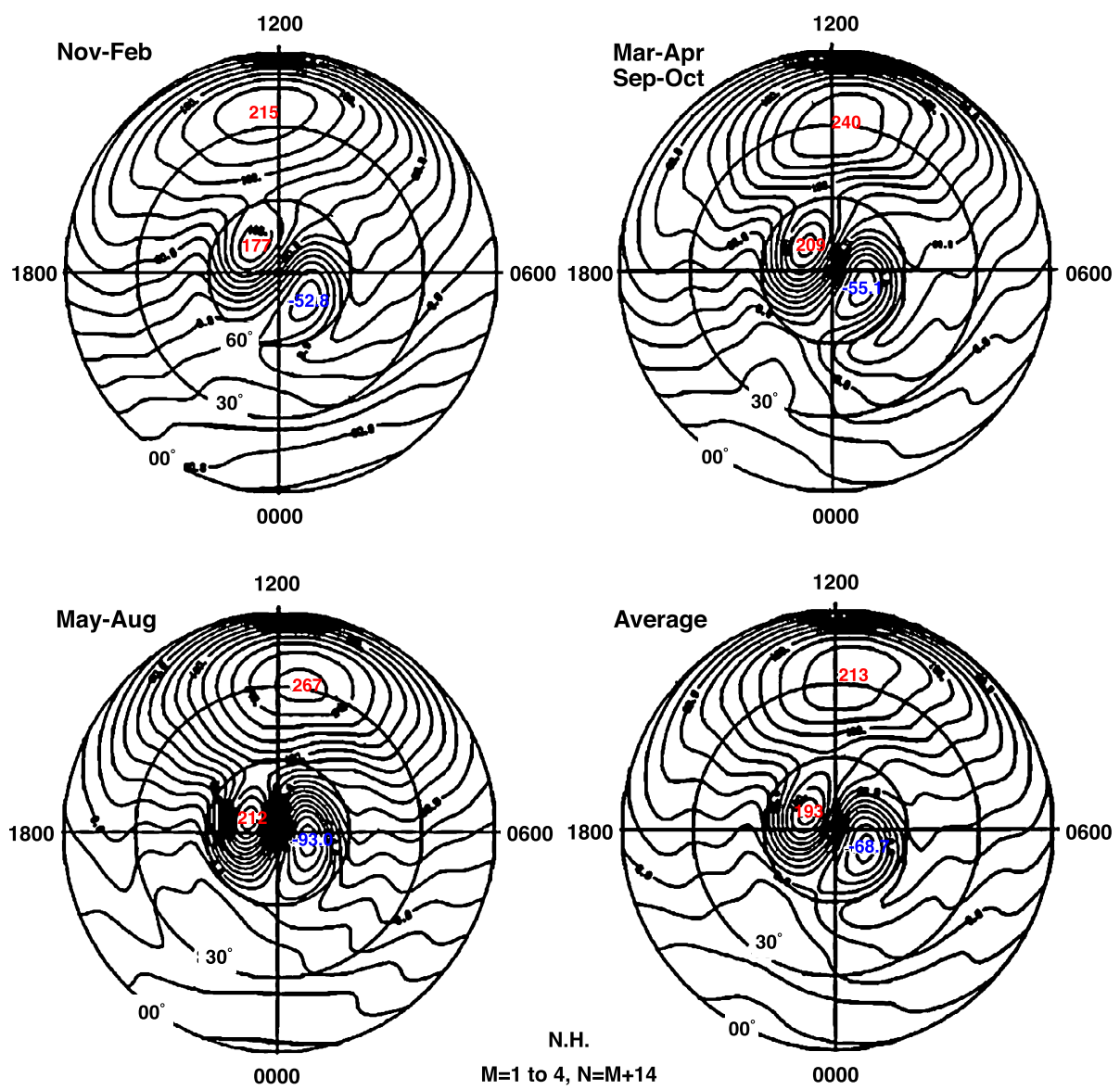

Fig. 4 Average external current systems in the Northern Hemisphere during Jun 1957-December 1959. The contour intervals are $20 \mathrm{kA}$. From Matsushita and Xu (1982)

due to in-situ solar heating on the dayside. The negative-mode diurnal tide was independently discovered by Lindzen (1966) in the same year.

Early simulations of the ionospheric dynamo (Stening 1969; Tarpley 1970; Richmond et al. 1976; Forbes and Lindzen 1976a) demonstrated that the negative-mode diurnal tide can generate a global electric current system similar to those derived from Sq variations. Thus, the negative-mode diurnal tide was considered to be the primary driver of the Sq currents. Later, Richmond and Roble (1987), Yamazaki and Richmond (2013), and Yamazaki et al. (2014b) numerically evaluated the importance of upward-propagating tides from the lower atmosphere. These upward-propagating tides are generated in the stratosphere by absorption of solar radiation by ozone, and in the troposphere by absorption of infrared radiation and latent heat release (Zhang et al. 2010a, 2010b, and references therein). As the waves propagate upward from the source regions, they grow exponentially with height, and at dynamo region altitudes, they attain amplitudes of several tens of meters per second in the horizontal wind. Yamazaki and Richmond (2013), and Yamazaki et al. (2014b) showed that about one third of Sq currents and about one-half of EEJ are driven by the upward-propagating tides. That 

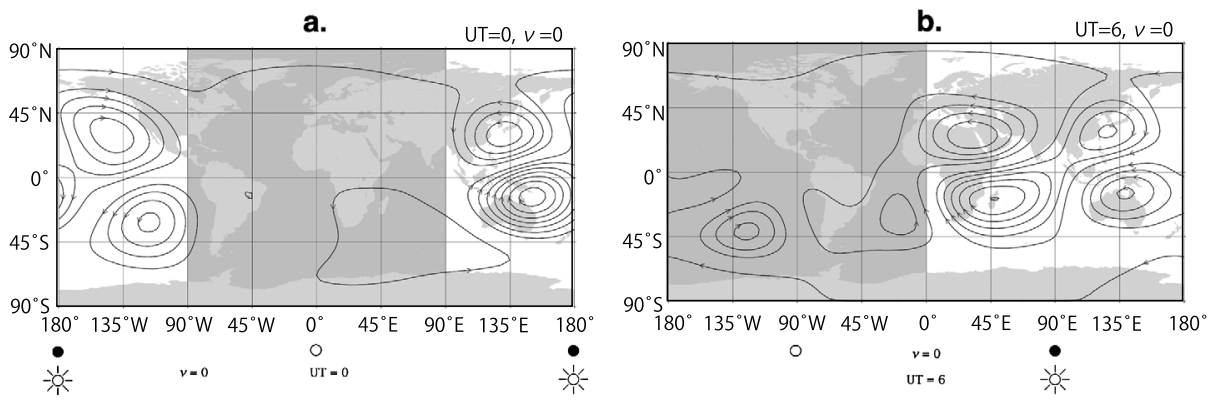

C.
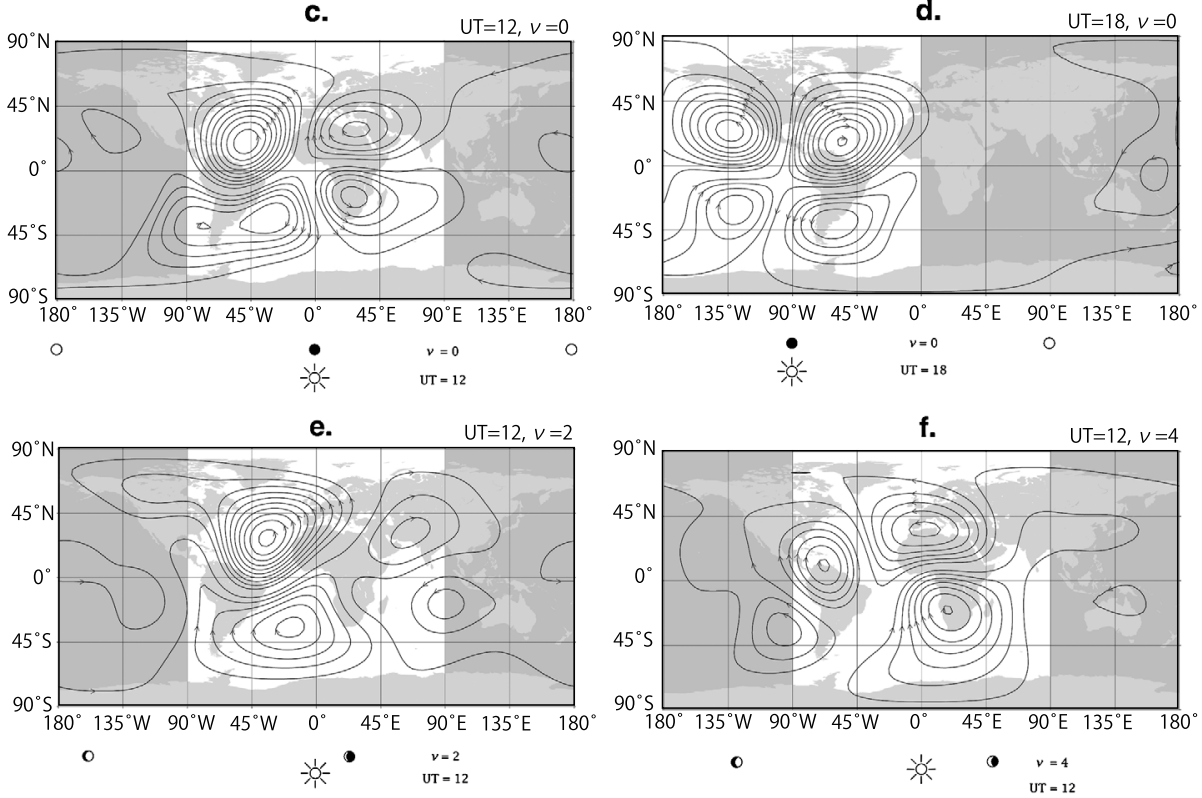

Fig. 5 External equivalent L current systems for various combinations of universal time (UT) and lunar age $v$ : (a) $\mathrm{UT}=0, v=0$, (b) $\mathrm{UT}=6, v=0,(\mathbf{c}) \mathrm{UT}=12, v=0,(\mathbf{d}) \mathrm{UT}=18, v=0$, (e) UT $=12, v=2$, and (f) $\mathrm{UT}=12, v=4$. The contour intervals are $1 \mathrm{kA}$. From Çelik (2014)

is, $\mathrm{Sq}$ variations are significantly affected by the tidal waves of lower atmospheric origin; this is why Sq studies are important for understanding of vertical atmospheric coupling.

\subsection{Lunar daily variations $L$}

The daily variation of the geomagnetic field contains a component that changes systematically with the phase of the moon, which is called lunar variation and often denoted as L. For the history of L, the reader is referred to the paper by Campbell (1980). Geomagnetic lunar variations are globally observed. Near the magnetic equator, an enhancement of the L field occurs, similar to Sq. L can be separated from Sq by a statistical analysis; see Sect. 3.10. Source currents for L exist in the dynamo region (90-150 km). Examples of equivalent current systems for L are shown in Fig. 5 (after Çelik 2014). The L current system typically consists of four current vortices, two in the Northern Hemisphere and the other two in the Southern Hemisphere. The main driving force for L currents is atmospheric 
lunar tides. Unlike atmospheric solar tides that are thermally excited by solar heating, atmospheric lunar tides are primarily caused by the gravitational pull of the moon exerting on the Earth's atmosphere. The gravitational force acts mostly on the bottom layer of the atmosphere where the mass of the atmosphere is concentrated. Lunar tidal waves are also produced by the tidally induced movement of the solid Earth and oceans (Hollingsworth 1971), and the redistribution of Earth/ocean mass associated with these motions. Similar to solar tides, atmospheric lunar tides propagate vertically upward. The amplitude of the wave grows exponentially with height, and the maximum amplitude is attained at $\sim 120 \mathrm{~km}$ where damping due to eddy and molecular dissipation occurs (Vial and Forbes 1994; Pedatella et al. 2012a). Atmospheric lunar tides are dominated by the semidiurnal (12.421hr) component. According to the satellite measurements reported by Zhang and Forbes (2013), semidiurnal lunar tidal winds in the dynamo region have peak amplitudes of $15-20 \mathrm{~m} / \mathrm{s}$, which is remarkably consistent with the earlier estimates by Maeda and Fujiwara (1967) based on the analysis of geomagnetic lunar variations. The amplitude of L is typically one tenth of the amplitude of Sq (e.g., Yamazaki et al. 2011b). The smaller amplitude of L is owing to weaker driving winds. In the dynamo region, the amplitude of solar semidiurnal tidal winds is approximately twice as large as that of lunar semidiurnal tidal winds. Also, while the negative-mode diurnal tide plays a role in Sq currents, there is no corresponding tidal mode for L. Nonetheless, the geomagnetic L variation has occasionally been observed to have an unusually large amplitude, even exceeding the apparent amplitude of Sq. Those big L days are often found at equatorial latitudes during Northern Hemisphere winter, and occasionally during the equinoxes (Bartels and Johnston 1940; Onwumechili 1964). Recent studies pointed out that the big L days during the Northern Hemisphere winter are often associated with the occurrence of stratospheric sudden warming events (Fejer et al. 2010; Yamazaki 2014). The effect of stratospheric sudden warming on Sq and $\mathrm{L}$ will be detailed in Sect. 4.3.3.

\section{Production Mechanisms}

\subsection{Dynamo Theory}

The ionospheric wind dynamo, or ionospheric dynamo for short, is a key concept for understanding the production mechanism of Sq and $\mathrm{L}$ currents (see reviews in Table 1). It describes electric fields and currents that result from the motion of the neutral atmosphere. Although the motion of neutrals itself does not cause any electromagnetic field, electrodynamic effects arise through the collisions between neutral and plasma particles. The ionospheric dynamo usually refers to the dynamo process at E-region heights. The dynamo process in the F-region ionosphere (>150 km), or F-region dynamo, is detailed by Maute and Richmond (2016) in this issue.

Figure 6 is a flow diagram describing how electric currents are generated in the ionosphere by the action of neutral winds (after Vasyliūnas 2012). In the dynamo region of the ionosphere $(90-150 \mathrm{~km})$, the ion-neutral collision frequency is comparable with or larger than the gryo-frequency of ions while the electron-neutral collision frequency is much smaller than the gyro-frequency of electrons. Thus, ions are coupled with neutral winds while electrons are frozen to magnetic field lines. The bulk motion of the plasma is driven by collisions between ions and neutrals. The plasma flow generates an electric field $\mathbf{E}=-\mathbf{V} \times \mathbf{B}$, where $\mathbf{V}$ is the plasma flow velocity and $\mathbf{B}$ is the geomagnetic field. The electric field induced by the plasma flow is generally not curl-free, so that the magnetic field 
Fig. 6 A schematic diagram for the development of the ionospheric wind dynamo. From Vasyliūnas (2012)

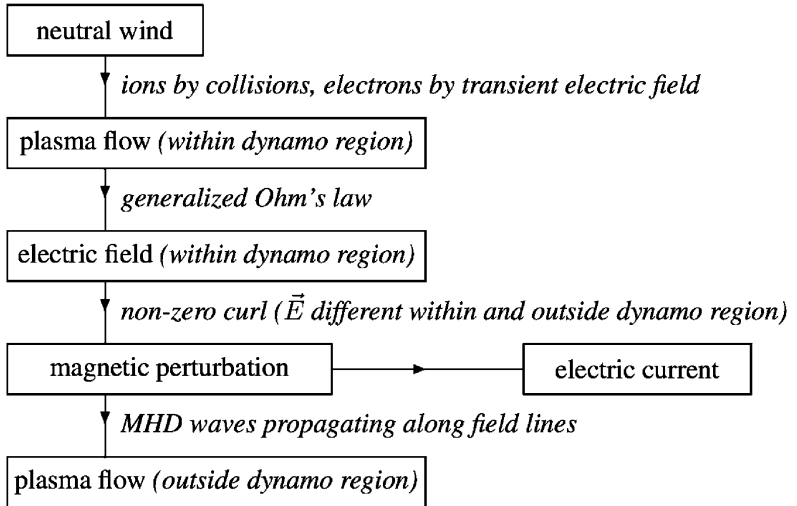

perturbation $\mathbf{b}$ arises according to Faraday's law $\nabla \times \mathbf{E}=-\partial \mathbf{b} / \partial t$. The deformation of the magnetic field relates to ionospheric currents $\mathbf{J}$ through Ampère's law $\nabla \times \mathbf{b}=\mu_{0} \mathbf{J}$, where $\mu_{0}$ is the vacuum permeability.

For the purpose of global ionospheric modeling, which deals with phenomena on time scales longer than one minute, the steady state assumption is valid. Ohm's law relates the steady-state electric current density $\mathbf{J}$ with the conductivity tensor $\boldsymbol{\sigma}$, electric field $\mathbf{E}$, neutral wind $\mathbf{U}$, and geomagnetic field $\mathbf{B}$ as follows:

$$
\mathbf{J}=\boldsymbol{\sigma}(\mathbf{E}+\mathbf{U} \times \mathbf{B}) .
$$

The current density is required to be divergence-free,

$$
\nabla \cdot \mathbf{J}=0
$$

and the static electric field can be expressed by an electric potential $\Phi$ :

$$
\mathbf{E}=-\nabla \Phi
$$

In the ionosphere, the conductivity tensor is highly anisotropic. The conductivity in the direction of the geomagnetic field is many orders of magnitude greater than the conductivities perpendicular to the geomagnetic field. Accordingly, ionospheric electric fields and currents are strongly organized by the geomagnetic field. Using the ionospheric conductivity parallel to the geomagnetic field $\left(\sigma_{\|}\right)$and those perpendicular to it $\left(\sigma_{P}\right.$ and $\left.\sigma_{H}\right),(1)$ becomes

$$
\mathbf{J}=\sigma_{\|} \mathbf{E}_{\|}+\sigma_{P}\left(\mathbf{E}_{\perp}+\mathbf{U} \times \mathbf{B}\right)+\sigma_{H} \frac{\mathbf{B}}{|\mathbf{B}|} \times\left(\mathbf{E}_{\perp}+\mathbf{U} \times \mathbf{B}\right),
$$

where $\mathbf{E}_{\|}$and $\mathbf{E}_{\perp}$ represent the component of the electric field parallel and perpendicular to the geomagnetic field, respectively. $\sigma_{\|}, \sigma_{P}$ and $\sigma_{H}$ are called parallel, Pedersen, and Hall conductivities, respectively. For currents perpendicular to $\mathbf{B}$, the Pedersen conductivity gives the component in the direction of the electric field and the Hall conductivity gives the component perpendicular to both electric field and geomagnetic field. Although there is no direct measurement of the ionospheric conductivities, one can derive them from a combination of measurable parameters and theoretical consideration. Maeda (1977) and Takeda and Araki (1985) introduced the following formula:

$$
\sigma_{\|}=\frac{e^{2} n_{e}}{m_{e} v_{e}},
$$




$$
\begin{gathered}
\sigma_{P}=\sigma_{\|} \frac{(1+\kappa) v_{e}^{2}}{(1+\kappa)^{2} v_{e}^{2}+\Omega_{e}^{2}}, \\
\sigma_{H}=\sigma_{\|} \frac{\Omega_{e} v_{e}}{(1+\kappa)^{2} v_{e}^{2}+\Omega_{e}^{2}}
\end{gathered}
$$

with

$$
\begin{gathered}
\Omega_{e}=\frac{e|\mathbf{B}|}{m_{e}}, \quad \Omega_{i}=\frac{e|\mathbf{B}|}{m_{i}}, \\
v_{e}=v_{e n}+v_{e i}, \quad \kappa=\frac{\Omega_{i} \Omega_{e}}{v_{e} v_{i n}},
\end{gathered}
$$

where $n_{e}$ is electron density; $v_{e n}$ is electron-neutral collision frequency; $v_{e i}$ is electronion collision frequency; $v_{i n}$ is ion-neutral collision frequency; $\Omega_{e}$ is electron cyclotron frequency; $\Omega_{i}$ is ion cyclotron frequency; $e$ is the elementary charge; $m_{e}$ is the electron mass. Figure 7 shows typical midlatitude daytime ionospheric conductivities as a function of height. These results are obtained from the ionospheric conductivity model of the World Data Center for Geomagnetism, Kyoto, which is based on (5)-(8). Since the parallel conductivity is very large throughout the ionosphere, the parallel electric field is almost completely shorted out. It is, therefore, reasonable to assume that geomagnetic field lines are equipotential. This means that the electric potential is constant along geomagnetic field lines all the way from the bottom of the ionosphere in one hemisphere to the bottom of the ionosphere in the opposite hemisphere, so that electric fields can be mapped between any pair of conjugate points.

For given neutral winds, ionospheric conductivity and geomagnetic field, one can solve (2)-(4) to calculate electric fields and currents. Review articles by Richmond (1989) and Richmond and Maute (2014) discuss various techniques of the ionospheric wind dynamo modeling. Figure 8 shows neutral winds, height-integrated Pedersen conductivity, electrostatic potential, and equivalent current function, derived from the National Center for Atmospheric Research thermosphere ionosphere electrodynamics general circulation model (NCAR TIE-GCM) (Richmond et al. 1992; Qian et al. 2014). The results are shown for geomagnetically quiet conditions for the September equinox of 2009 and 2002, representing solar minimum and solar maximum, respectively. Each panel of Fig. 8 represents a snap shot at $1200 \mathrm{UT}$, so that the Greenwich longitude $\left(0^{\circ} \mathrm{E}\right)$ is in the midday. Neutral winds at $110 \mathrm{~km}$ (Figs. 8a and 8b) are dominated by upward-propagating tides from the lower atmosphere. It can be seen that the semidiurnal tide is the major component of the wind field at

Fig. 7 Height profiles of parallel, Pedersen and Hall conductivities calculated for a mid-latitude location $\left(35^{\circ} \mathrm{N}, 135^{\circ} \mathrm{E}\right)$ under the noon-time March equinox condition for low solar activity, using the ionospheric conductivity model of the World Data Center for Geomagnetism, Kyoto

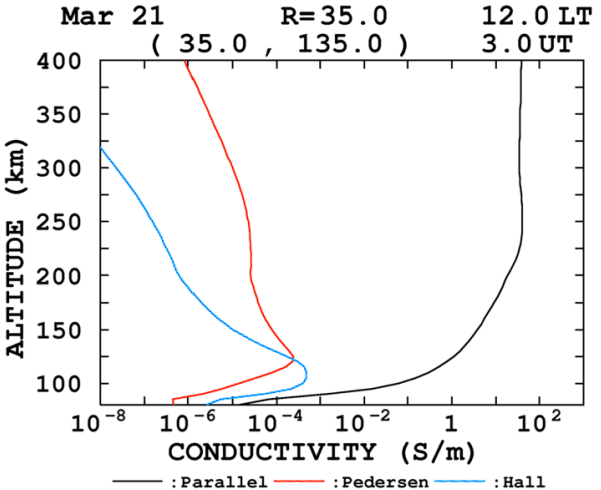



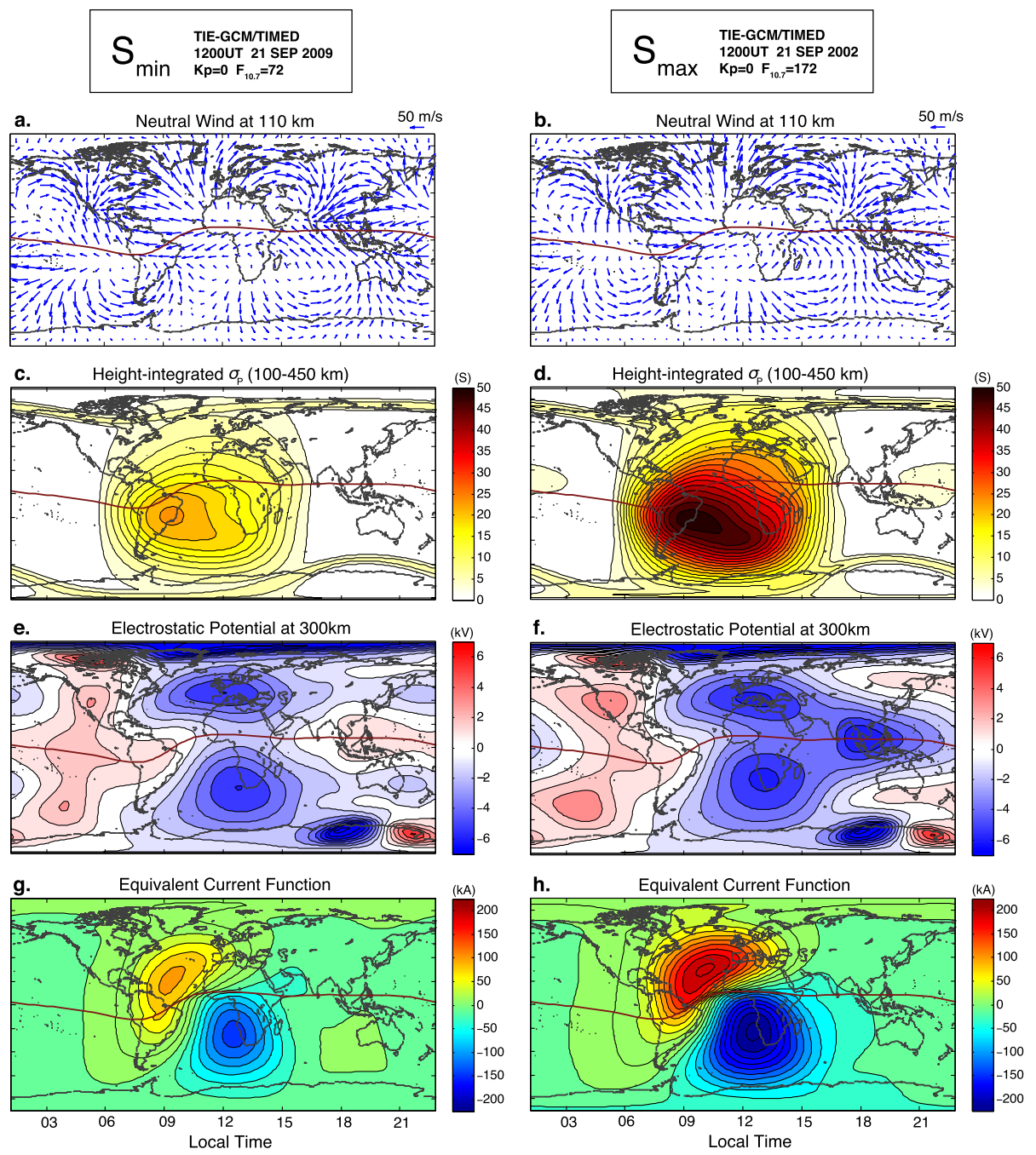

Fig. 8 TIE-GCM simulation results for magnetically quiet equinox conditions $(K p=0)$, at 1200 UT, for (left) low solar activity $\left(F_{10.7}=72 \mathrm{sfu}\right)$ and (right) high solar activity $\left(F_{10.7}=172 \mathrm{sfu}\right)$. (a) $-(\mathbf{b})$ Neutral winds at $110 \mathrm{~km}$. (c)-(d) Hight-integrated Pedersen conductivity. (e)-(f) Electrostatic potential at $300 \mathrm{~km}$. (g)-(h) Equivalent current function at $110 \mathrm{~km}$

this particular height. In general, neutral winds below $\sim 150 \mathrm{~km}$ are dominated by the atmospheric tides of lower atmospheric origin. Meanwhile, neutral winds in the region above are dominated by the negative-mode diurnal tides, which are locally generated by in-situ solar heating. In Figs. $8 \mathrm{a}$ and $8 \mathrm{~b}$, the wind patterns are remarkably consistent between the solar minimum and solar maximum cases. Upward propagating tides from the lower atmosphere are not sensitive to the solar cycle (e.g., Oberheide et al. 2009).

The height-integrated Pedersen conductivity (Figs. 8c and 8d) is much greater during the day than night, as the conductivity varies with electron density. In addition, the conductivity varies inversely with the strength of the background geomagnetic field (see (5)-(8)). 
This explains relatively high conductivities around the South Atlantic Anomaly, in which the geomagnetic field is weakest. At high latitudes, there are bands of elevated ionospheric conductivity along the auroral oval due to ionization caused by energetic particle precipitation. The electric potential (Figs. 8e and 8f) is symmetric about the magnetic equator at middle and low latitudes, as the model assumes equipotential magnetic field lines. The electric potential is largely independent of solar activity, except that during solar maximum there is a rapid potential drop at the magnetic equator around the sunset. This corresponds to the pre-reversal enhancement of the zonal electric field, which is often observed in the equatorial ionosphere during solar maximum (e.g., Woodman 1970; Fejer et al. 1979; Farley et al. 1986). The electric potential in the polar cap is associated with magnetospheric convection electric fields, which are transmitted to the high-latitude ionosphere along equipotential geomagnetic field lines. The DP2 current system (see Fig. 4) is essentially Hall currents driven by the high-latitude electric field. Finally, the equivalent Sq current system (Figs. 8g and 8h) strongly depends on solar activity. The solar activity dependence is largely due to ionospheric conductivities. Takeda et al. (2003) confirmed this by comparing $\mathrm{Sq}$ variations with electric field measurements from the MU radar and ionospheric conductivities from an empirical model. The Sq current system is not symmetric about the magnetic equator even in this equinox simulation because of the asymmetry in neutral winds and conductivities.

\subsection{Inter-Hemispheric Field-Aligned Currents}

The north-south asymmetry in neutral winds and ionospheric conductivities indicates an asymmetric dynamo action between conjugate points. Although most models of the ionospheric dynamo assume equipotential geomagnetic field lines $\left(\sigma_{\|}=\infty\right)$, the parallel conductivity has a finite value in the real ionosphere. Thus, a fractional difference can exist in the electric potential between the conjugates, which produces inter-hemispheric field-aligned currents. In the global models that assume $\sigma_{\|}=\infty$, the inter-hemispheric field-aligned currents cannot be calculated from Ohm's law (4). Instead, the parallel current density at the top of the ionosphere is derived from the condition that the three dimensional current has to be divergence-free. That is, the divergence and convergence of horizontal currents in the ionosphere must be balanced by downward and upward currents that flow between the two hemispheres along the geomagnetic field.

The presence of the inter-hemispheric field-aligned currents was first suggested by van Sabben (1966) in an attempt to explain the north-south asymmetry of the equivalent Sq current system. Subsequent studies numerically evaluated inter-hemispheric field-aligned currents (van Sabben 1969, 1970; Schieldge et al. 1973; Maeda 1974; Stening 1977a; Takeda 1982). These studies predicted that (1) the asymmetric dynamo can cause the interhemispheric field-aligned currents with the maximum current density of some $10^{-2} \mu \mathrm{A} / \mathrm{m}^{2}$ and that (2) the direction of the inter-hemispheric field-aligned currents depends on local time. Figure 9a is a sketch of the inter-hemispheric field-aligned currents by Fukushima (1994).

Early observational evidence for the inter-hemispheric field-aligned currents includes an intrusion of the Sq current system from the Northern Hemisphere into the Southern Hemisphere (or the other way around) across the magnetic equator. The equivalent $\mathrm{Sq}$ current systems in Fig. 3 clearly show such cross-equatorial currents in June and September. The inter-hemispheric field-aligned currents such as those in Fig. 9 would cause "apparent" cross-equatorial currents in the equivalent Sq current system. Simulation studies confirmed that the inter-hemispheric field-aligned currents are partly responsible for the 
Fig. 9 (a) Schematic illustrating inter-hemispheric field-aligned currents at middle latitudes, from Fukushima (1994). (b) The dependence of inter-hemispheric field-aligned currents on magnetic local time, from Lühr et al. (2015). Positive values represent currents from the Southern Hemisphere to the Northern Hemisphere.

(c) TIE-GCM simulation results for quiet equinox conditions with $F_{10.7}=120 \mathrm{sfu}$.

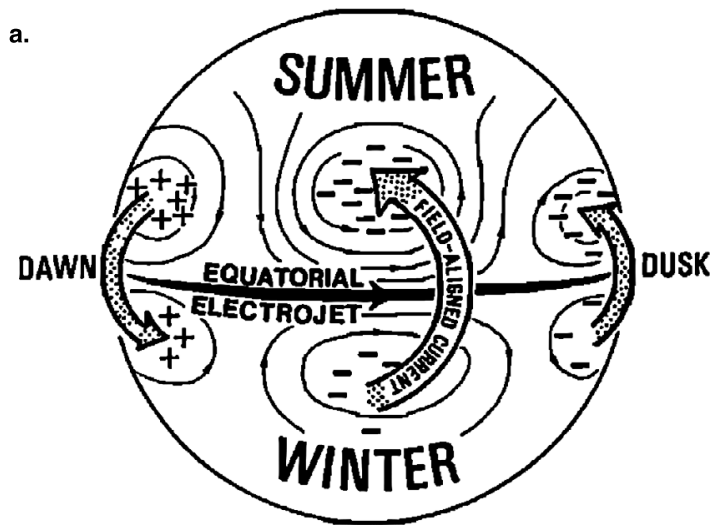

b.

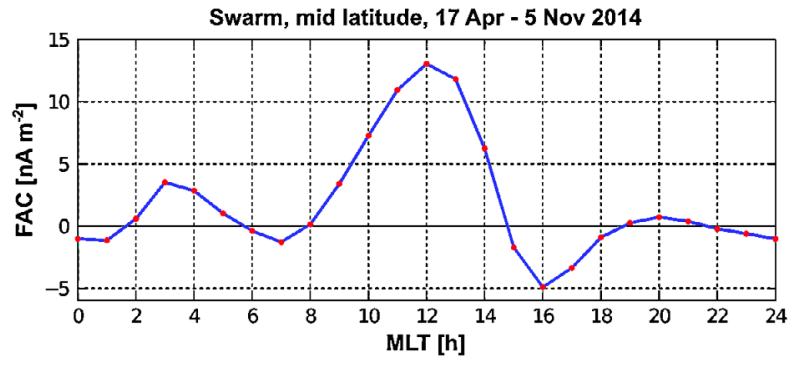

c.

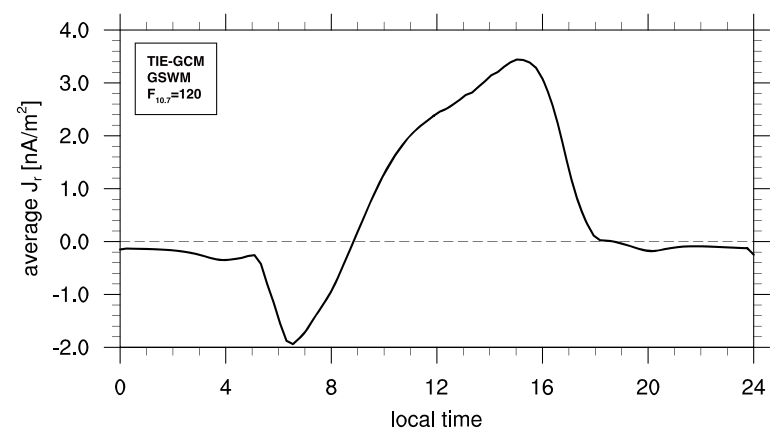

cross-equatorial currents in the equivalent Sq current system (Richmond and Roble 1987; Takeda 1990).

Olsen (1997b) presented the first direct evidence of the inter-hemispheric field-aligned currents using the magnetic data from the Magsat satellite. Yamashita and Iyemori (2002) analyzed magnetic data from the Ørsted satellite to study the seasonal climatology of the inter-hemispheric field-aligned currents. Park et al. (2011) showed a comprehensive seasonal, local-time and longitudinal climatology of the inter-hemispheric field-aligned currents based on 9 years of magnetic field measurements by the CHAMP (CHAllenging Minisatellite Payload) satellite. Lühr et al. (2015) used measurements from the Swarm satellite constellation. Three satellites of the Swarm constellation can directly measure the curl of the vector magnetic field, which makes it possible to derive the inter-hemispheric field- 
aligned currents without assuming the structure of the currents. Figure $9 \mathrm{~b}$ is from Lühr et al. (2015) showing the inter-hemispheric field-aligned currents observed by the Swarm constellation during 17 April-5 November 2014 as a function of magnetic local time. The results involve the measurements at $20^{\circ}-40^{\circ}$ magnetic latitudes from both the Northern and Southern Hemispheres. In the figure, positive values indicate currents flowing from the Southern Hemisphere to the Northern Hemisphere. Strong northward currents around noon, as well as weak southward currents in the morning, are consistent with Fukushima's prediction (Fig. 9a). Also, TIE-GCM simulation results (Fig. 9c) are in qualitative agreement with the observations.

\subsection{Cowling Effect}

The Cowling effect (Cowling 1933) is the mechanism in which the Pedersen conductivity is effectively enhanced in the presence of boundaries that restricts Hall currents and causes a polarization electric field. The effect is often mentioned in explaining the equatorial electrojet, but it is also important for the global Sq currents. A strong Cowling effect occurs at the magnetic equator where the geomagnetic field is horizontal (Hirono 1950; Baker and Martyn 1953). Figure 10 illustrates the Cowling effect at the magnetic equator. During daytime, the ionospheric electric field usually points eastward above the magnetic equator ( $\mathbf{E}_{\mathbf{1}}$ in Fig. 10). This gives rise to an eastward Pedersen current $\left(\sigma_{P} \mathbf{E}_{\mathbf{1}}\right)$, along with a downward Hall current $\left(\sigma_{H} \mathbf{E}_{\mathbf{1}}\right)$. The downward flow of the Hall current is inhibited by the presence of poorly conducting layers above and below the dynamo region, which leads to charge accumulation on the boundaries. Consequently, an upward polarization electric field is set up ( $\mathbf{E}_{\mathbf{2}}$ in Fig. 10). The upward electric field produces not only an upward Pedersen current $\left(\sigma_{P} \mathbf{E}_{2}\right)$ that balances with the downward Hall current $\left(\sigma_{H} \mathbf{E}_{\mathbf{1}}\right)$, but also an eastward Hall current $\left(\sigma_{H} \mathbf{E}_{\mathbf{2}}\right)$. The net effect is a strong eastward current $\left(\sigma_{P} \mathbf{E}_{\mathbf{1}}+\sigma_{H} \mathbf{E}_{\mathbf{2}}\right)$. From the balance of the vertical currents,

$$
\sigma_{H} \mathbf{E}_{\mathbf{1}}=\sigma_{P} \mathbf{E}_{\mathbf{2}} .
$$

Thus, the net eastward current can be expressed as

$$
\sigma_{P} \mathbf{E}_{\mathbf{1}}+\sigma_{H} \mathbf{E}_{\mathbf{2}}=\left(\sigma_{P}+\frac{\sigma_{H}^{2}}{\sigma_{P}}\right) \mathbf{E}_{\mathbf{1}}
$$

The effective zonal conductivity $\sigma_{P}+\sigma_{H}^{2} / \sigma_{P}$ is called Cowling conductivity. Numerical studies have shown that the vertical currents in the equatorial electrojet are not completely inhibited. Rather, vertical currents are connected to meridional currents, forming a verticalmeridional current loop on both sides of the equatorial electrojet (Untiedt 1967; Sugiura and Poros 1969; Richmond 1973; Forbes and Lindzen 1976b; Takeda and Maeda 1980a). During the daytime, currents flow equatorward at $95-130 \mathrm{~km}$, upward over the magnetic equator, poleward at $130-150 \mathrm{~km}$, and downward several degrees from the magnetic equator.

Fig. 10 Schematic illustrating the Cowling effect in the equatorial electrojet. From Yamazaki (2011)

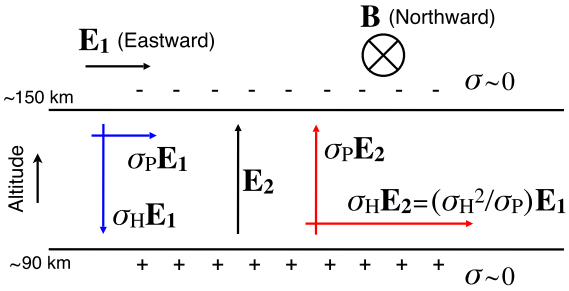


Fig. 11 Equivalent Sq current systems computed (top) with and (bottom) without Hall conductivity. From Takeda (1991)
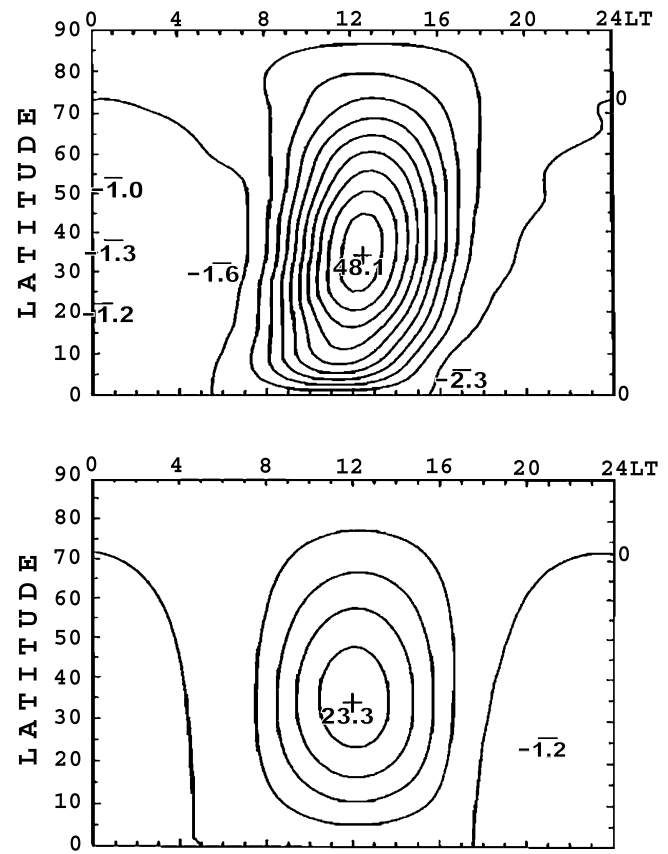

Alken and Maus (2010) examined the relationship between the eastward electric field and the strength of the equatorial electrojet. The EEJ strength tends to increase with increasing eastward electric field, but the linear relationship breaks down when the electric field is strong. According to Alken and Maus (2010), those observations can be explained as the effect of ionospheric plasma instabilities (namely, two-stream and gradient-drift instabilities). These plasma instabilities become active under the presence of strong vertical electric field $(\geq 2 \mathrm{mV} / \mathrm{m})$ and reduce the vertical polarization electric field ( $\mathbf{E}_{\mathbf{2}}$ in Fig. 10).

Fukushima (1979) pointed out that Pedersen and Hall currents tend to flow in the same direction in Sq currents. That is, the global Sq current system, as well as the equatorial electrojet, is under the Cowling effect. His idea was supported by simulation results by Takeda (1991) based on an ionospheric dynamo model. Figure 11 shows equivalent Sq current systems simulated (top) with and (bottom) without Hall conductivity (after Takeda 1991). The inclusion of the Hall conductivity does not alter the pattern of the Sq current system but it increases the total current intensity by a factor of 2 . This means that the Hall current flows in the same direction as the Pedersen current, acting to enhance the effective Pedersen conductivity; this is exactly how the Cowling effect works. It is noted that the Cowling effect is much more effective at the magnetic equator than middle and low latitudes. At the magnetic equator, the Hall current contribution to the total current is $\sim 10$ times the Pedersen current contribution, while at higher latitudes, the Hall current contribution is comparable to the contribution of the Pedersen current.

\subsection{Counter Electrojet}

The equatorial electrojet is usually eastward during the daytime. However, there are occasionally days when geomagnetic data indicate a westward current flow, typically lasting for a few hours. The reversed flow of the equatorial electrojet is called counter electrojet 
(CEJ), or reverse electrojet (REJ). Main characteristics of CEJ can be found in review articles by Mayaud (1977) and Marriott et al. (1979). Various mechanisms have been suggested to explain CEJ. Modeling studies have shown that a particular combination of tidal modes can result in a counter electrojet (Forbes and Lindzen 1976b; Takeda and Maeda 1981; Hanuise et al. 1983). The study performed by Raghavarao and Anandarao (1980) showed that an upward wind with a sufficiently large magnitude $(15-20 \mathrm{~m} / \mathrm{s})$ can also cause a counter electrojet. Observational studies have found that the occurrence of CEJ is dependent on the phase of the moon, suggesting that lunar tides play a role (Bartels and Johnston 1940; Rastogi 1974; Sastri and Arora 1981). Other studies found that a large-amplitude CEJ event during winter is often observed during a stratospheric sudden warming event, suggesting a physical connection between the two phenomena (Stening et al. 1996; Sridharan et al. 2009; Fejer et al. 2010); see Sect. 4.3.3 for more discussion on the stratospheric sudden warming effect on Sq and EEJ.

A quiet-time CEJ event is often accompanied by unusual Sq variations at other latitudes, indicating that a CEJ is a result of a large-scale process (Stening 1977b; Bhargava and Sastri 1977). Global ionospheric current systems during the occurrence of CEJ events were examined by several authors (e.g., Takeda and Maeda 1980b; Rastogi 1994; Gurubaran 2002; Yamazaki et al. 2012a). Those studies revealed the presence of additional current systems that are superposed on the normal Sq current system.

During geomagnetically active periods, a counter electrojet is sometimes caused by the penetration of the polar-region electric field to equatorial latitudes (Rastogi and Patel 1975; Rastogi 1977, 1997; Kikuchi et al. 2003, 2008). Besides, storm-time thermospheric winds tend to drive a westward electric field in the dayside equatorial region through the mechanism known as disturbance dynamo (Blanc and Richmond 1980; Fuller-Rowell et al. 2002). The effect of the disturbance dynamo reduces or sometimes even reverses the eastward electric field produced by normal quiet-time winds. The disturbance dynamo electric field can persist for several days after geomagnetic activity quiets down, and thus can result in a reduced EEJ or CEJ during the recovery phase of a storm (Le Huy and Amory-Mazaudier 2005; Yamazaki and Kosch 2015). More discussion on the storm-time response of $\mathrm{Sq}$ and EEJ can be found in Sect. 4.3.4.

Recently, Vineeth et al. (2016) reported a remarkable correlation between the monthly mean meteor counts and the number of afternoon CEJ events during 2006-2007. Their observations are consistent with the numerical results by Muralikrishna and Kulkarni (2008), which predicted that a dust-particle layer of meteoric origin could cause a reversal of the vertical polarization electric field in the equatorial electrojet.

\subsection{Sq-EEJ Relationship}

The relationship between the global Sq current system and the equatorial electrojet has been a matter of debate for decades. Some researchers see the equatorial electrojet as merely an equatorial portion of the global Sq current system (e.g., Stening 1995a). Others believe that the equatorial electrojet forms its own current system independent of the global Sq current system (e.g., Onwumechili 1992). Figure 12 is a sketch presented by Ogbuehi et al. (1967) for (a) the global Sq current system and (b) the current system associated with the equatorial electrojet. In this section, we will take a close look at the debate on the Sq-EEJ relationship. It should be noted that the goal of this section is not to discuss which idea is right, but rather to recognize that there is more than one perspective from which the data can be interpreted.

When Chapman (1951) coined the term 'equatorial electrojet', he defined it as concentrated eastward electric currents "superposed on the normal Sq currents". That is, the 
Fig. 12 Schematic drawings of (a) global Sq current system and (b) equatorial electrojet current system. From Ogbuehi et al. (1967)

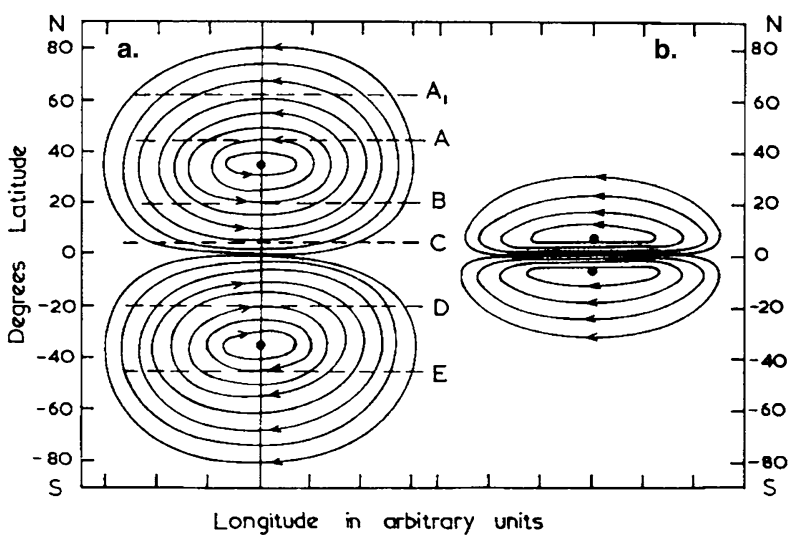

equatorial electrojet was originally thought to be separate from $\mathrm{Sq}$ currents. The name 'equatorial electrojet' obviously comes from the analogy to the auroral electrojet. It is reasonable to consider that the auroral electrojet is separate from $\mathrm{Sq}$ currents, because their driving mechanisms are distinctively different. The auroral electrojet is driven by the solar-wind magnetospheric dynamo, while Sq currents are driven by the ionospheric wind-dynamo. Another example for the separation of two current systems is Sq and L. Sq currents and L currents are separable, because Sq currents are driven by thermallyexcited solar tides, while L currents are driven by gravitationally-excited lunar tides. These examples for the separation of two current systems are based on the fact that two phenomena have separate production mechanisms; in other words, they can exist independently. In this light, Stening (1995a) asked: what drives the equatorial electrojet? Evidence suggests that the equatorial electrojet is driven by the eastward electric field resulting from the ionospheric dynamo at middle and low latitudes (Stening 1977c; $\mathrm{Du}$ and Stening 1999). This means that a substantial portion of the equatorial electrojet could not exist without the global Sq current system. Thus, one may reasonably ask: what is the physical significance of the Sq-EEJ separation? Perhaps, some might argue that the EEJ is a Hall current $\left(\sigma_{H} \mathbf{E}_{\mathbf{2}}\right.$ in Fig. 10) while Sq is a Pedersen current $\left(\sigma_{P} \mathbf{E}_{\mathbf{1}}\right.$ in Fig. 10). However, as is obvious from Fig. 11, the Hall current does not form such a current system as that illustrated in Fig. 12b. Now, one may ask again: should we not separate Sq and EEJ? As will be shown below, the Sq-EEJ separation is practically useful, as it often makes data processing simple and easy. For this reason, many researchers routinely separate Sq and EEJ without addressing its physical meaning.

Early studies on the Sq-EEJ relationship examined the correlation in the daily range of the horizontal magnetic field observed at an "equatorial station", located within the narrow band of the equatorial electrojet ( $\pm 3^{\circ}$ from the magnetic equator) and an "off-equatorial station" at the same longitude, located several degrees away from the equatorial electrojet. In many cases, poor correlation was found (Osborne 1963, 1966, 1968; Ogbuehi et al. 1967; Schlapp 1968; Mann and Schlapp 1988; Manoj et al. 2006; Abdul Hamid et al. 2014). The results were often taken as evidence that the equatorial electrojet is not a simple enhancement of Sq currents and hence other driving mechanisms exist for the EEJ. Yamazaki et al. (2014a) noted, however, that the poor correlation in $H$ between equatorial and off-equatorial stations can be reproduced by a numerical model in which the equatorial electrojet is treated as a part of the global wind dynamo current system. Therefore, different behaviors of $H$ at equatorial and off-equatorial stations do not necessarily mean that $\mathrm{Sq}$ and EEJ have separate driving mechanisms. 
a.

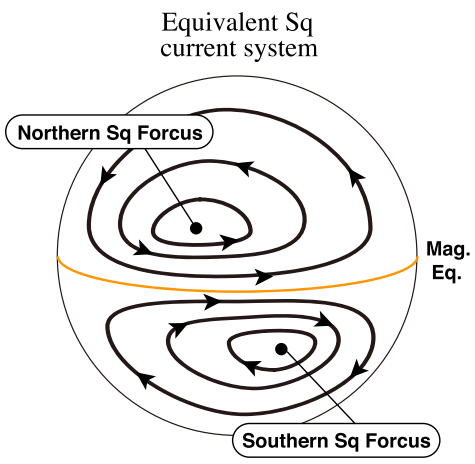

c.

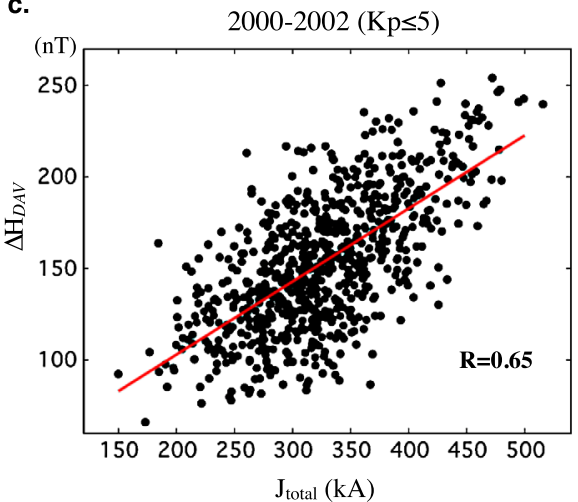

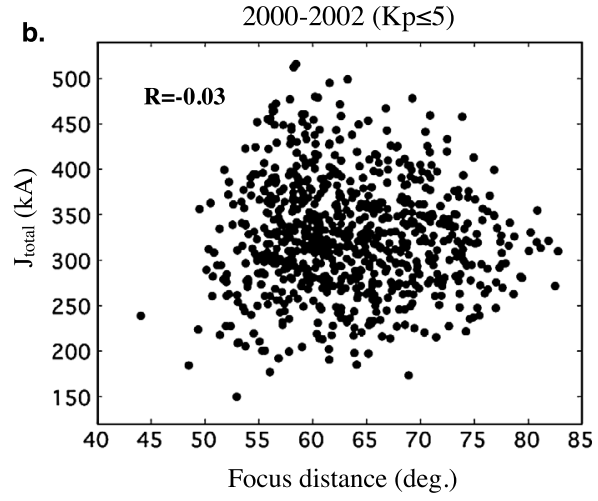

d.

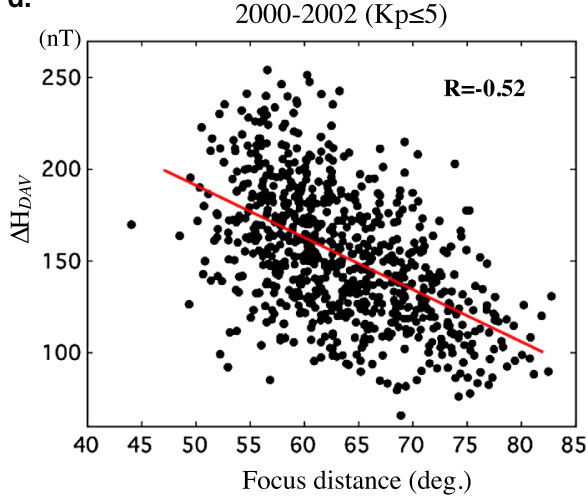

Fig. 13 (a) Schematic illustrating the dayside view of the equivalent Sq current system. (b) The relationship of the total Sq current intensity $J_{\text {total }}$ and the spherical distance between the northern and southern Sq foci. Scatter plots for (c) the daily range in $H$ at Davao and $J_{\text {total }}$, and for (d) the daily range in $H$ at Davao and the $\mathrm{Sq}$ focus distance. From Yamazaki (2011)

Yamazaki (2011) performed a spherical harmonic analysis (see Sect. 3.7) of Sq variations in the East Asian sector to evaluate global Sq current systems from 2000 through 2002, and examined how they relate to the daily range of $H$ at the equatorial station Davao (DAV, $\left.7.0^{\circ} \mathrm{N}, 125.4^{\circ} \mathrm{E}\right)$. (The Davao data were not included in the spherical harmonic analysis.) Two parameters were derived from the equivalent $\mathrm{Sq}$ current system on the daily basis. One is the total Sq current intensity (see Sect. 3.8), which is the total amount of E-region (90-150 km altitudes) currents flowing in the region between the northern and southern Sq foci (Fig. 13a). The other parameter is the spherical distance between the northern and southern Sq foci. As shown in Fig. 13b, the two quantities do not correlate. This means that the intensity and shape of the Sq current system can change independently. Figure 13c shows that the daily range of $H_{\mathrm{DAV}}$ tends to be larger when the intensity of the global Sq current system is larger. Meanwhile, Fig. $13 \mathrm{~d}$ reveals that the daily range of $H_{\mathrm{DAV}}$ tends to be larger when the Sq current foci are closer. These results suggest that the daily range of $H_{\mathrm{DAV}}$ depends not only on the intensity but also on the shape of the global Sq current system. A large portion of the variability in the daily range of $H_{\mathrm{DAV}}$ can be explained if the variability of both intensity and shape of the global Sq current system is taken into account. Other studies have also recognized the importance of the spatial variation of the 

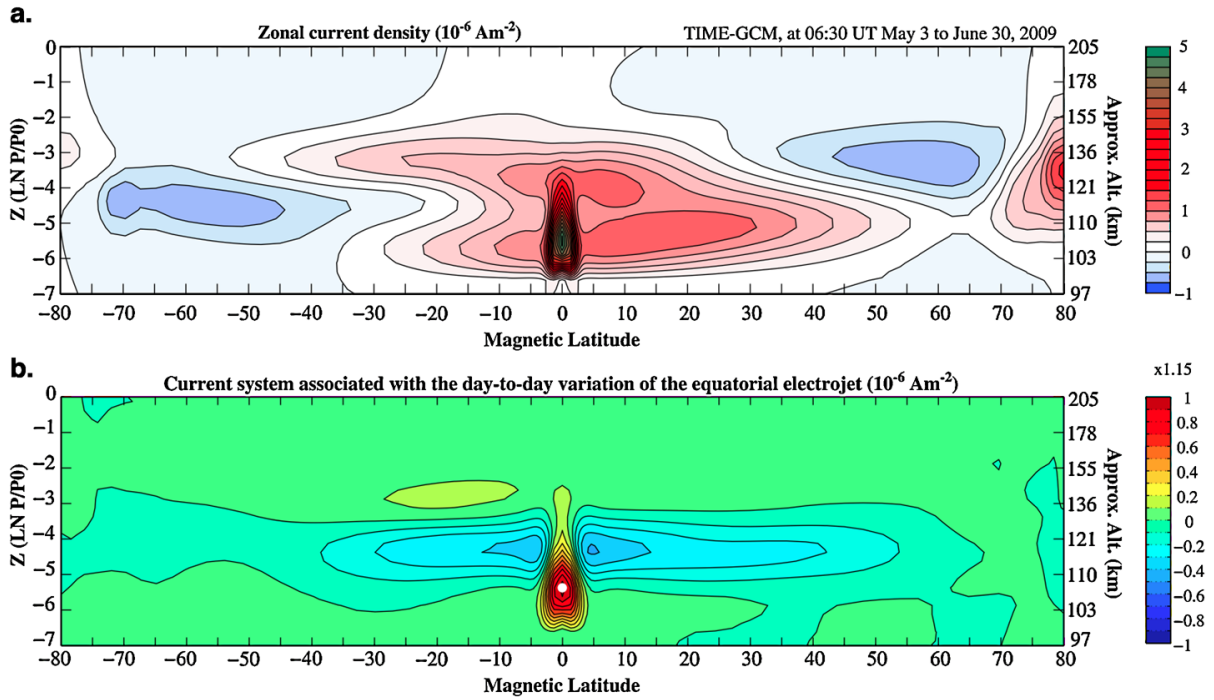

Fig. 14 Simulated noon-time eastward current density in the Indian sector during May-June 2009, from Yamazaki et al. (2014a). (a) The average current density. (b) The current system associated with the dayto-day variation of the peak electrojet current. The results are shown as a function of magnetic latitude and height. $Z$ is the constant pressure interface $\left(=\ln \left(P_{0} / P\right)\right.$, where $P$ is the atmospheric pressure and $\left.P_{0}=5 \times 10^{-10} \mathrm{hPa}\right)$. The approximate altitudes are also indicted

Sq current system for the variability of the equatorial electrojet (Kane 1971, 1974; Tarpley 1973; Rajaram 1983; Yamazaki et al. 2010).

Now let us consider whether an ionospheric current system such as that in Fig. 12b can possibly exist. The answer is "Yes". Figure 14a, from a simulation study by Yamazaki et al. (2014a), shows the midday cross section of the eastward current density over the Indian sector, averaged over the period from 3 May through 30 Jun 2009. The equatorial electrojet is evident as an enhanced eastward current flow over the magnetic equator with the peak current density approximately $5 \times 10^{-6} \mathrm{~A} / \mathrm{m}^{2}$ at $105 \mathrm{~km}$. Currents are eastward at low latitudes (within $\pm 30^{\circ}$ magnetic latitudes) and westward at higher latitudes, representing a global Sq current system. Figure 14b depicts the ionospheric current system associated with the day-to-day variation of the equatorial electrojet, derived using the technique introduced by MacDougall (1979a, 1979b). The results reveal westward "return" currents at the flanks of the electrojet current. The current pattern is consistent with that in Fig. 12b. Yamazaki et al. (2014a) explained the formation of this current system (Fig. 14b) as follows. When neutral winds drive electric currents in the dynamo region, they tend to produce a polarization electric field that opposes the currents. For example, if neutral winds drive a westward current at low latitudes, it tends to produce an eastward polarization electric field. The eastward polarization electric field spreads in latitude beyond the generation region and drives an eastward electrojet current at the magnetic equator. Thus, perturbations in the wind-driven currents at low latitudes negatively correlate with perturbations in the equatorial electrojet. As noted by Yamazaki et al. (2014a), the current system in Fig. 14b explains only less than $25 \%$ of the total current shown in Fig. 14a. Thus, the mechanism described above operates only partially. The rest of the east equatorial electrojet is likely to connect to the westward Sq currents on the polar side of Sq foci, but this needs to be confirmed in future studies. 
In a recent study, Vichare et al. (2016) took a new approach to the problem of the SqEEJ relationship. They used a stochastic method based on transfer entropy, which enabled to quantify the information shared or exchanged by magnetic signals at equatorial and offequatorial stations. It was found that significant information is exchanged between $\mathrm{Sq}$ and EEJ, indicating that EEJ and Sq are a coupled system. Interestingly, their results showed that the information tends to be transferred from EEJ to Sq, rather than Sq to EEJ.

Although the physical significance of the Sq-EEJ separation is debatable, it is often convenient to treat the data as if there are two separable contributions from Sq and EEJ. The simplest way to separate Sq and EEJ is to take the difference in $H$ between equatorial and off-equatorial stations. If there exist separable Sq and EEJ current systems such as those in Fig. 12, $\mathrm{H}$ at the equatorial station will contain the effect of both $\mathrm{Sq}$ and EEJ. Meanwhile, $H$ at the off-equatorial station will represent the pure Sq effect because it is located near the focus of the EEJ current system. Therefore, the difference in $H$ between the two stations contains only the EEJ effect. Because of the simplicity, the technique is widely used for studies of the equatorial electrojet (e.g., Anderson 2011; Yizengaw et al. 2014). The true advantage of this technique is not the separation of Sq but the separation of magnetospheric contributions. At both equatorial and off-equatorial stations, the $H$ field is constantly disturbed by the effect of magnetospheric currents. These disturbances are nearly identical at the two stations because the distance between the pair of stations (several hundreds of kilometers) is much smaller than the distance from magnetospheric currents to the stations (several Earth radii). Thus, taking the difference of the $H$ field at the two stations effectively eliminates the contribution of magnetospheric currents. Figure 15 displays a monthly record of the $H$-component geomagnetic field at (top) Alibag (ABG, $12.2^{\circ}$ magnetic latitude) and (middle) Trivandrum (TRD, $-0.2^{\circ}$ magnetic latitude) in October 1983. Although there was no severe storm during this month, geomagnetic activity was occasionally high. Consequently, both $H_{\mathrm{ABG}}$ and $H_{\mathrm{TRD}}$ are under the effect of magnetospheric currents $(\sim 50 \mathrm{nT})$ throughout the month. This makes it difficult to determine a suitable baseline for daily variations that is required for the calculation of ionospheric currents. The bottom panel of Fig. 15 shows the difference $H_{\mathrm{TRD}}-H_{\mathrm{ABG}}$. The nighttime level is now very constant, which can be used as the baseline.

The analysis of satellite magnetometer data also benefits from the Sq-EEJ separation. Unlike the analysis of ground magnetometer data, a satellite-data analysis usually requires a main geomagnetic field model to determine the baseline of the Sq field. Since the Sq field is much smaller than the main field, the use of the main field model brings much uncertainty in the Sq analysis; note also that there is an overlap of wavelength in the spatial variations of the main field and Sq field (Olsen and Stolle 2012). Nonetheless, a reliable baseline can be determined for EEJ, assuming that Sq and EEJ are separable. The Sq-EEJ separation is generally done by some type of curve fitting to the middle- and low-latitude data, which does not reflect any physical process. Figure 16 is an example of the curve fitting (Lühr et al. 2004). In the figure, the black line is the residual field after the subtraction of the main field model from the raw data, and the green line is a fit to the large-scale field. The green line separates Sq and EEJ contributions, and serves as a baseline for the EEJ field. Since the difference between the black and gray lines does not depend much on the main field model, the derived EEJ field is robust to the selection of the main field model. For this reason, the Sq-EEJ separation is a common practice for EEJ studies based on satellite data analysis (e.g., Le Mouël et al. 2006; England et al. 2006; Manoj et al. 2006; Alken and Maus 2007; Tomás et al. 2008; Alken et al. 2015). 
Fig. 15 The horizontal $(H)$ component geomagnetic field at Alibag $\left(18.6^{\circ} \mathrm{N}, 72.9^{\circ} \mathrm{E}\right)$ and Tirunelveli $\left(8.7^{\circ} \mathrm{N}, 77.8^{\circ} \mathrm{E}\right)$, India in October 1983
Fig. 16 An example of the Sq-EEJ separation in the satellite magnetometer data. From Lühr et al. (2004)
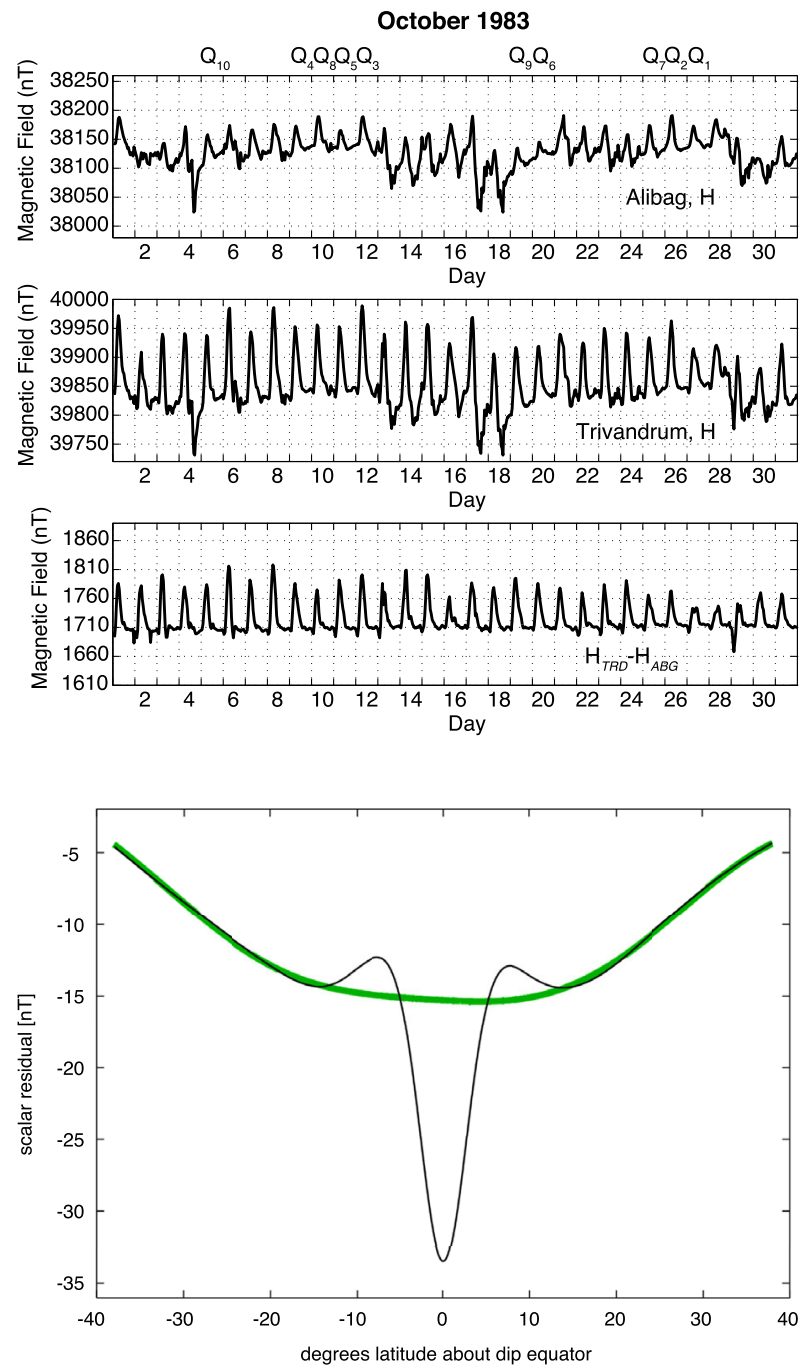

\section{Data Analysis Techniques}

\subsection{Selection of Quiet Days}

Quiet days are when a smooth regular daily variation dominates the daily record of the geomagnetic field, while disturbed days are when the geomagnetic record is dominated by irregular fluctuations. The selection of quiet days is important for most Sq studies. A common practice for defining quiet days is to use the five (or ten) International Quiet Days (IQDs), which are selected by the GFZ Potsdam based on the $K p$ index. In Figs. 1 and 15, the ten IQDs of the month are indicated by $\mathrm{Q}_{1}, \mathrm{Q}_{2}, \ldots$, and $\mathrm{Q}_{10}$. Since IQDs are merely "quietest" days of the month, they are not necessarily disturbance-free. The actual level of geomagnetic activity for IQDs varies with activity of the Sun. Consequently, IQDs during active solar periods are not as quiet as IQDs during quiet solar periods (Campbell 1979). 
Table 2 Daily maximum $K p$ value and occurrence rate
S.N. represents the annual sunspot number

\begin{tabular}{lrrr}
\hline$K p$ & All & S.N. $<30$ & S.N. $>150$ \\
\hline 0 & $0.1 \%$ & $0.2 \%$ & $0.0 \%$ \\
$\leq 0^{+}$ & $0.6 \%$ & $1.1 \%$ & $0.1 \%$ \\
$\leq 1^{-}$ & $2.0 \%$ & $3.8 \%$ & $0.5 \%$ \\
$\leq 1$ & $4.5 \%$ & $8.0 \%$ & $1.8 \%$ \\
$\leq 1^{+}$ & $8.5 \%$ & $14.0 \%$ & $4.3 \%$ \\
$\leq 2^{-}$ & $13.7 \%$ & $21.3 \%$ & $7.7 \%$ \\
$\leq 2$ & $20.2 \%$ & $29.9 \%$ & $12.9 \%$ \\
$\leq 2^{+}$ & $27.7 \%$ & $38.3 \%$ & $19.4 \%$ \\
$\leq 3^{-}$ & $36.4 \%$ & $47.6 \%$ & $27.4 \%$ \\
$\leq 3$ & $45.7 \%$ & $56.4 \%$ & $36.8 \%$ \\
$\leq 3^{+}$ & $55.0 \%$ & $65.0 \%$ & $46.0 \%$ \\
$\leq 4^{-}$ & $63.6 \%$ & $72.7 \%$ & $55.4 \%$ \\
$\leq 4$ & $71.1 \%$ & $79.2 \%$ & $64.1 \%$ \\
$\leq 4^{+}$ & $77.4 \%$ & $84.2 \%$ & $71.3 \%$ \\
$\leq 5^{-}$ & $82.9 \%$ & $88.8 \%$ & $77.6 \%$ \\
$\leq 5$ & $87.3 \%$ & $92.2 \%$ & $82.6 \%$ \\
$\leq 5^{+}$ & $90.8 \%$ & $94.9 \%$ & $86.6 \%$ \\
$\leq 6^{-}$ & $93.3 \%$ & $96.8 \%$ & $89.7 \%$ \\
$\leq 6$ & $95.3 \%$ & $98.1 \%$ & $92.2 \%$ \\
\hline
\end{tabular}

An alternative way of defining quiet days is to introduce $K p$ criteria in selecting the data. In this approach, the largest value of the eight $K p$ indices of a day is restricted to a certain level (e.g., less than 3), and all the days that do not satisfy this condition are excluded from the analysis. This guarantees that the selected days are as geomagnetically quiescent as intended. It is important to ensure that geomagnetic activity is lower than the target level not only during the daytime but also during the nighttime, because the nighttime data are normally used to determine the baseline for Sq variations (see Sect. 3.2).

Table 2 shows the occurrence rate of quiet days for different $K p$ criteria. The results are based on the $K p$ index during 1932-2015. For example, approximately one third of days meets the condition that the maximum $K p$ value is less than or equal to $2^{+}$. Table 2 also shows how the data availability varies between solar maximum years and solar minimum years for the same $K p$ criteria. Here, the solar maximum years are defined as the years with the average sunspot number being greater than 150 , while the solar minimum years are those with the average sunspot number being less than 30 . The $K p$ criteria need to be selected depending on the purpose of use. In some studies, using only disturbance-free data is important, while in other studies, having continuous data is more important than having clean data. Table 3 shows examples of the $K p$ criteria used in various Sq studies.

Some studies used multiple criteria for the selection of quiet days. For example, Yamazaki (2014) defined quiet days as those with $K p \leq 2^{+}$for the present day and $K p<4$ for the previous day. By avoiding the days that follow a disturbed day, one can avoid the effect of storm-time winds, which the $K p$ index is not sensitive to. Disturbance winds can persist for more than a day after a geomagnetic storm (Huang et al. 2005a). Other studies employed $A E$ and $D s t$ indices in order to define more strictly quiet days (Campbell 1979; Torta et al. 1997). 
Table $3 K p$ criteria used in various studies

Fig. 17 Geomagnetic field perturbations in $X, Y$, and $Z$ at Chichijima $\left(27.1^{\circ} \mathrm{N}, 142.2^{\circ} \mathrm{E}\right)$, along with the Dst index, during March 1990. From Yamada (2002)

\begin{tabular}{ll}
\hline Abdul Hamid et al. (2014) & $K p \leq 3$ \\
Campbell (1981) & $K p \leq 3^{+}$ \\
Campbell( 1982, 2003) & $K p \leq 2^{+}$ \\
Campbell (1989b) & $K p<3$ \\
Campbell and Matsushita (1982) & $K p<2^{+}$ \\
Campbell and Schiffmacher (1985) & $K p<3$ \\
Campbell et al. (1993) & $K p \leq 2^{-}$ \\
Shinbori et al. (2014) & $K p<4$ \\
Stening et al. (2007) & $K p \leq 2$ \\
Takeda (2002a, 2002b, 2013a) & $K p \leq 3^{+}$ \\
Takeda (2013b) & $K p<3^{+}$ \\
Yamada (2002, 2009) & $K p \leq 5^{+}$ \\
Yamazaki et al. (2011a) & $K p \leq 5$ \\
Yamazaki et al. (2010, 2011b) & $K p \leq 2^{+}$ \\
Yamazaki et al. (2012c) & $K p \leq 3^{+}$ \\
\end{tabular}

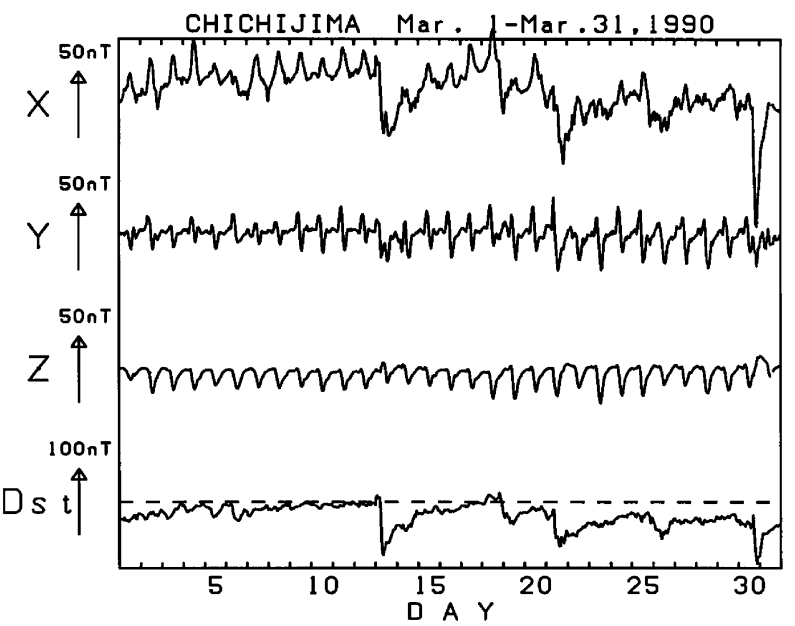

At high latitudes (above $60^{\circ}$ magnetic latitudes), the geomagnetic field is almost always disturbed and a visual inspection of the data is recommended for the selection of quiet days. Some authors have developed automated techniques to determine high-latitude Sq variations (e.g., Janzhura and Troshichev 2008; Stauning 2011).

Another factor to be considered for the selection of quiet days is that different components of the geomagnetic field have different sensitivity to geomagnetic activity. Among the three components of the geomagnetic field $X, Y$, and $Z$, the northward $X$ component is the most susceptible to geomagnetic disturbances at middle and low latitudes. The eastward $Y$ and vertical $Z$ components are much less affected. This is well illustrated in Fig. 17 (from Yamada 2002). Large disturbances in the $X$ component are due to various types of magnetospheric currents (Campbell 2004). Disturbances in the $Z$ component are relatively small, reflecting the fact that the conducting Earth tends to shield vertical magnetic disturbances imposed by magnetospheric currents. The judgement of quiet days should be made based 
on the most disturbed component if more than one element of the geomagnetic field is used in the analysis.

\subsection{Determination of Baselines}

Our next goal is to determine the baseline or zero level of Sq variations. In early studies of Sq, the mean (or median) value of the Sq variation was often used as the baseline. Later, however, it was realized that the average of nighttime values of the Sq variation is more physically meaningful because the source currents in the ionosphere effectively vanish during the night (e.g., Price and Wilkins 1963; Malin and Gupta 1977). At altitudes 100-150 km, nighttime Pedersen and Hall conductivities are a few orders of magnitude smaller than daytime values. The Sq current systems simulated by the NCAR TIE-GCM in Figs. 8g and $8 \mathrm{~h}$ are confined to the dayside, confirming the validity of the assumption that little current flows on the nightside.

The following are some examples of the nighttime baseline in the literature: Schlapp (1968) used the hourly value nearest to local midnight as the baseline; Rabiu et al. (2007) and Bolaji et al. (2015) used the average value for the two hours starting from local midnight; Siddiqui et al. (2015a) used two hours before midnight and two hours after midnight; Pham Thi Thu et al. (2011) used one hour before midnight and two hours after midnight; El Hawary et al. (2012) used one hour before midnight and three hours after midnight; Takeda (1984), Malin and Winch (1996), Yamazaki et al. (2012b) used the five hours nearest to local midnight.

At this stage, one may also remove the non-cyclic variation from the data, which is defined as a difference between the geomagnetic field at the preceding and succeeding local midnights (Chapman and Bartels 1940, p. 546). The non-cyclic variation arises as the geomagnetic field does not usually return to exactly the same value in 24 hours. Let $V_{t}$ denote the hourly value at local time $t(=0,1,2,3, \ldots, 24)$. The last value $V_{24}$ is the first value for the next day. If the midnight value $V_{0}$ is chosen to be the nighttime baseline, the non-cyclic variation is $\left(V_{24}-V_{0}\right) / 24=Q$ per hour. The $Q$ value may be computed for each day. The corrected hourly data are $V_{t}^{\prime}=V_{t}-Q t$; so that $V_{0}^{\prime}=V_{24}^{\prime}$. Alken et al. (2013) used cubic splines, instead of linear regressions, in order to represent the non-cyclic variation. They first fitted the cubic splines to the nighttime data (2200 to 0500 LT) and then subtracted those fits from all the data. The non-cyclic variation is mainly due to slowly varying magnetospheric currents during the recovery phase of a geomagnetic storm (Price 1963). Therefore, it is most pronounced in the $H$ component, and can be largely eliminated by applying the $D s t$ correction, which will be described in Sect. 3.3.

It may be noted that the assumption of zero current at night is not valid at high latitudes, where $\mathrm{Sq}^{p}$ currents and aurora electrojet exist. For studies on high-latitude $\mathrm{Sq}$ variations, the daily average or monthly average of the magnetic field may be used as the baseline (Matsushita and Xu 1982; Weimer et al. 2010). Gjerloev (2012), on the basis of 1-min SuperMAG data (Gjerloev 2009), introduced a technique that can be used at all latitudes. In his technique, the baseline of $\mathrm{Sq}$ is essentially the mode value (i.e., the value that appears most often in the data) within \pm 8 days, which at low latitudes, usually appears during night.

\subsection{Correction for the Dst Field}

The disturbance in the $H$ component of the geomagnetic field is dominated by the $D s t$ field, or the zonally symmetric disturbance field. It can be evaluated using the hourly $D s t$ 
index (or SYM-H index for 1-minute data) and removed from the $H$ data. At a station with geomagnetic latitude $\lambda$, the disturbance field $\delta_{H}$ relates to the $D s t$ index approximately by

$$
D s t=\frac{\delta_{H}}{\cos \lambda} .
$$

Thus, the disturbance field in $H$ can be corrected by subtracting $D s t \cdot \cos \lambda$. Takeda (1984, $1999,2002 \mathrm{a}, 2002 \mathrm{~b}$ ) attempted to remove the disturbance from the $Z$ component as well, by subtracting $0.25 D s t \cdot \sin \lambda$ from $Z$, where the constant value ' 0.25 ' comes from the induction effect.

As an alternative approach, Choudhary et al. (2011) first calculated a linear regression between $H$ and $D s t$, and then subtracted it from $H$. Here, we introduce a modified version of this Dst correction technique. Our intention is to correct the $H$ data for the Dst field and secular variation, and simultaneously determine the nighttime baseline. This will be achieved by fitting the Dst index and linear trend to the nighttime data of $H$ at a given station. The fitting equation may be represented as

$$
H^{n}=\alpha+\beta T^{n}+\gamma D s t^{n},
$$

where $T$ is time in Julian days. In (12), only the nighttime data are considered (e.g., five hourly values closest to the local midnight), which is indicated by the superscript $n$. In the following, we describe how to determine the fitting coefficients $\alpha, \beta$, and $\gamma$. Equation (12) can be expressed in the matrix form:

$$
\mathbf{d}^{n}=\mathbf{A}^{n} \mathbf{M},
$$

where $\mathbf{d}^{n}$ is a vector consisting of the $H$ data during the night time:

$$
\mathbf{d}^{n}=\left(\begin{array}{c}
H_{1}^{n} \\
H_{2}^{n} \\
\vdots \\
H_{k}^{n}
\end{array}\right),
$$

where $k$ is the total number of the nighttime data points. Given that the nighttime level changes seasonally (Campbell 1987), the suitable length of the data set for fitting would be between several days and a few months. In (13), $\mathbf{A}^{n}$ is a matrix of the model functions,

$$
\mathbf{A}^{n}=\left(\begin{array}{ccc}
1 & T_{1}^{n} & D s t_{1}^{n} \\
1 & T_{2}^{n} & D s t_{2}^{n} \\
\vdots & \vdots & \vdots \\
1 & T_{k}^{n} & D s t_{k}^{n}
\end{array}\right)
$$

and $\mathbf{M}$ is a vector of the fitting coefficients

$$
\mathbf{M}=\left(\begin{array}{l}
\alpha \\
\beta \\
\gamma
\end{array}\right) .
$$

Once the matrices $\mathbf{d}^{n}$ and $\mathbf{A}^{n}$ are determined using the data, (13) can be solved for $\mathbf{M}$ as follows:

$$
\mathbf{M}=\left\{\left(\mathbf{A}^{n}\right)^{\mathrm{T}} \mathbf{A}^{n}\right\}^{-1}\left\{\left(\mathbf{A}^{n}\right)^{\mathrm{T}} \mathbf{d}^{n}\right\},
$$




\section{September 2009}
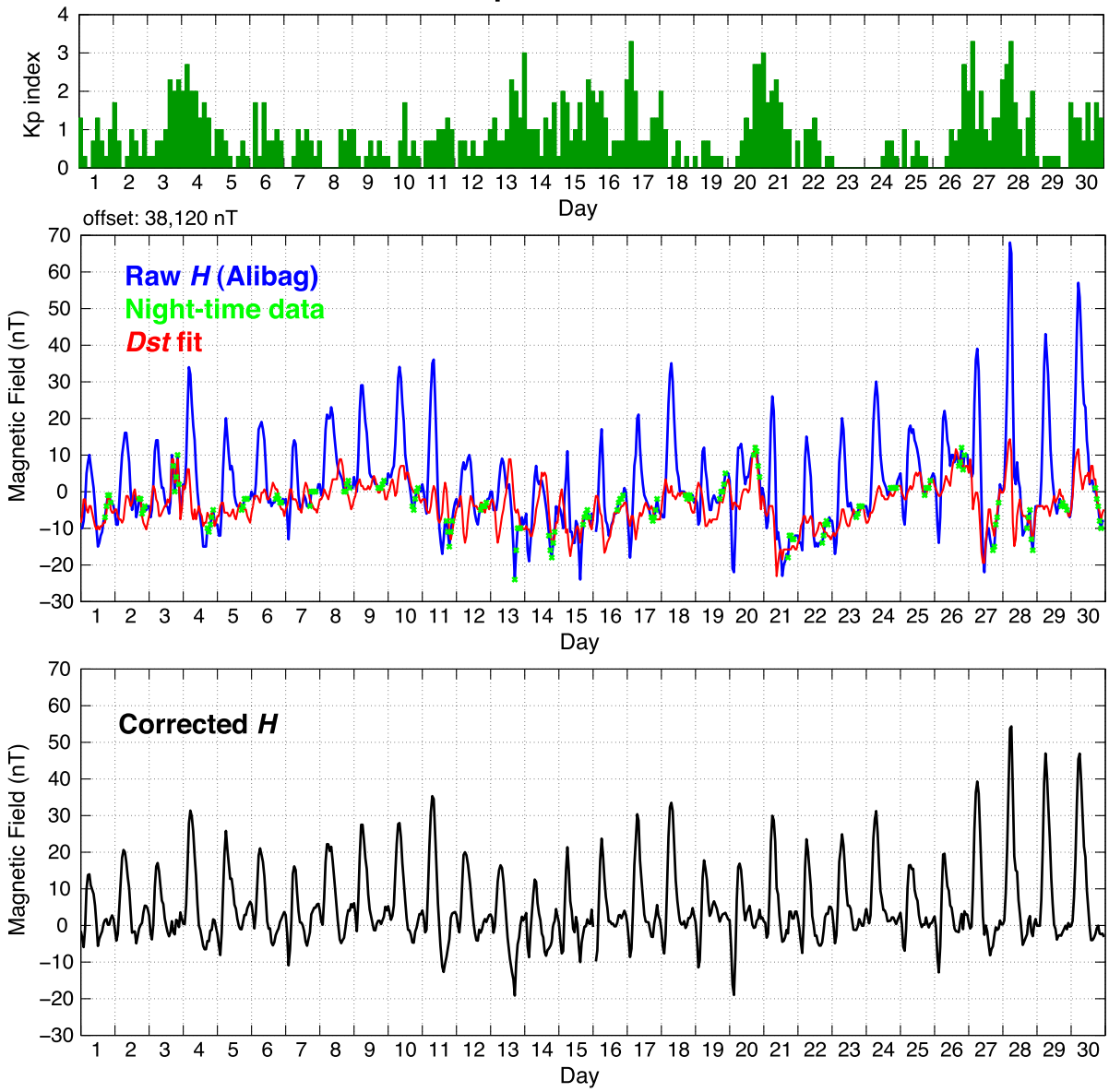

Fig. 18 An example of the Dst correction in $H$ at Alibag for September 2009 (solar minimum)

where the superscript $\mathrm{T}$ indicates the transpose of the matrix. Equation (17) represents the so-called least-squares solution, which gives the model coefficients that minimize the error between the model and observations. Once the coefficients $\alpha, \beta$, and $\gamma$ are determined, the daily variation in $H$ can be obtained from

$$
\Delta H=H-(\alpha+\beta T+\gamma D s t) .
$$

Also, the $D$ st-corrected field in $X$ and $Y$ can be derived as

$$
\begin{gathered}
X=(H-\gamma D s t) \cos D, \\
Y=(H-\gamma D s t) \sin D .
\end{gathered}
$$

Figure 18 presents an example of the Dst correction for $H$ at Alibag $\left(18.6^{\circ} \mathrm{N}, 72.9^{\circ} \mathrm{E}\right)$, India for September 2009. As the top panel shows, geomagnetic activity was very low throughout this month. In the middle panel, the raw $H$ data are plotted in blue, and the 

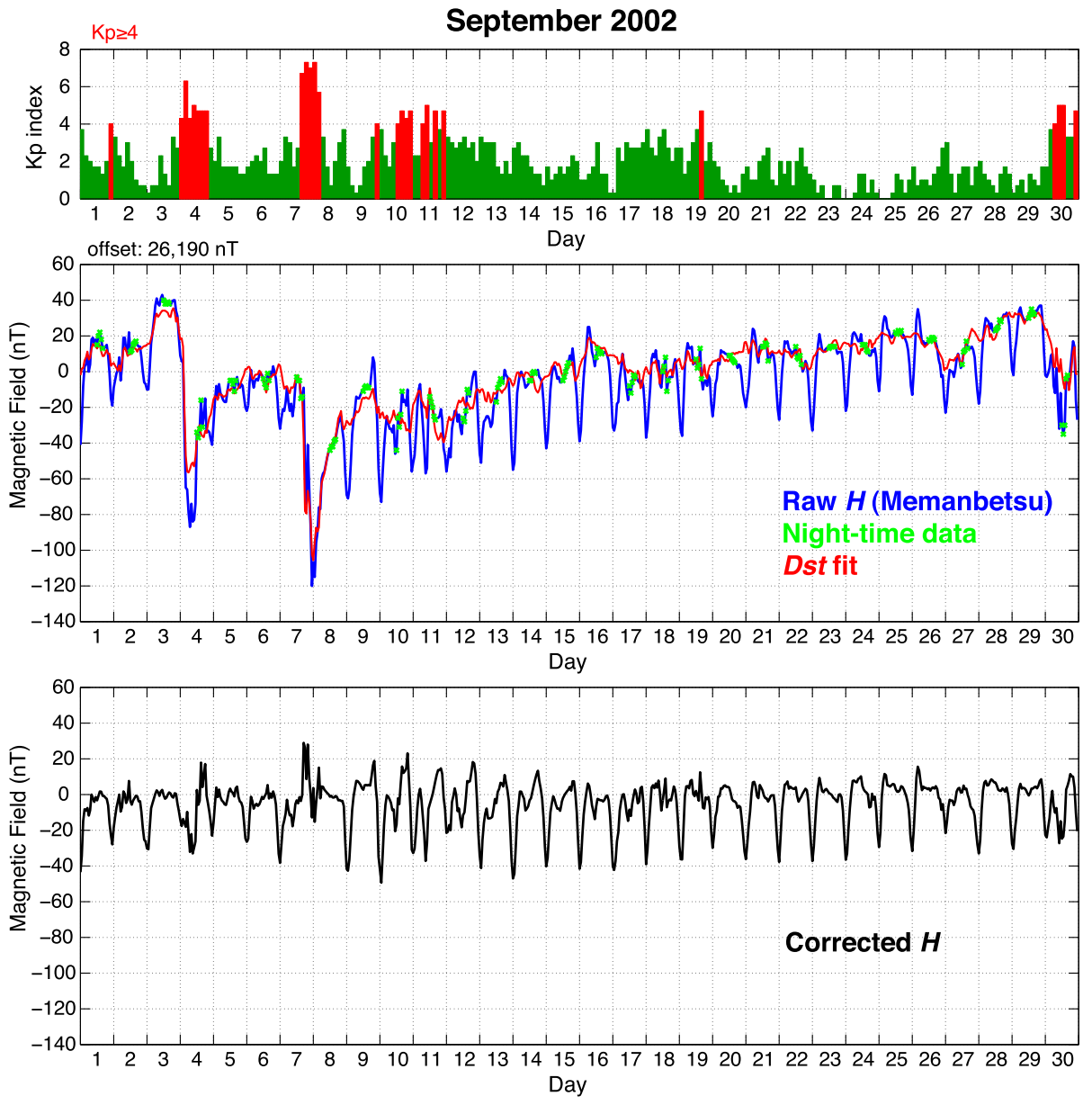

Fig. 19 An example of the Dst correction in $H$ at Memambetsu for September 2002 (solar maximum)

nighttime values, defined here as the five hourly values closest to the local midnight, are indicated by green. Despite the quiet condition of geomagnetic activity, the nighttime values of $H$ are not constant. Rather, they show the variation of up to $\pm 15 \mathrm{nT}$, which is comparable to the amplitude of Sq. The Dst fit (i.e., $\alpha+\beta T+\gamma D s t$ ), shown by red, closely follows the variation in the nighttime $H$. The bottom panel shows the corrected $H$. In Fig. 19, the same method was applied to $H$ at Memambetsu $\left(43.9^{\circ} \mathrm{N}, 144.2^{\circ} \mathrm{E}\right)$, Japan for September, 2002, representative of a geomagnetically active period. It is noted that the vertical scale of the plots is different from Fig. 18. There were two geomagnetic storms $(D s t<-100 \mathrm{nT})$ in September 2002. The Dst fit closely follows the nighttime $H$, even during the storm events, and the effect of these storms is much reduced in the corrected $H$. It is noted that the Dst correction does not remove all the disturbances in $H$. Rather, it removes only the disturbances that are zonally symmetric. In fact, the effect of stormtime magnetospheric currents exhibits a zonal asymmetry (e.g., Love and Gannon 2009; Newell and Gjerloev 2012), and the asymmetric disturbances will remain in the $H$ data after the $D s t$ correction. 


\subsection{Fourier Analysis}

Since the ionospheric dynamo currents are essentially solar driven, Sq variations are dominated by solar harmonic components. Fourier series expansion is, therefore, a suitable way to mathematically express $\mathrm{Sq}$ variations. In general, when geomagnetic disturbances are absent, the first four solar harmonics (i.e., 24-, 12-, 8-, and 6-hr components) are sufficient to capture most variability in a daily record of the geomagnetic field. That is,

$$
V_{t}=\sum_{m=0}^{4}\left\{a_{m} \cos \left(2 \pi \frac{m t}{24}\right)+b_{m} \sin \left(2 \pi \frac{m t}{24}\right)\right\}+V_{\text {base }}+Q t
$$

Here $V_{t}$ is the hourly value of the geomagnetic field in an arbitrary component at local time $t$; $V_{\text {base }}$ is the nighttime baseline; and $Q t$ is the non-cyclic variation. The Sq field at $t$ can be obtained as the perturbation from the baseline and non-cycle variation:

$$
\Delta V_{t}=V_{t}-V_{\text {base }}-Q t=a_{0}+\sum_{m=1}^{4}\left\{a_{m} \cos \left(2 \pi \frac{m t}{24}\right)+b_{m} \sin \left(2 \pi \frac{m t}{24}\right)\right\}
$$

The term $b_{0}$ is always zero and thus omitted. On the other hand, the term $a_{0}$ is necessary because the average of $\Delta V_{t}$ is generally not zero.

A least squares fitting technique is often used to find the harmonic coefficients $a_{m}$ and $b_{m}$ that minimize the error between $\Delta V_{t}$ and measurements. Using the matrix form, (22) can be written as

$$
\mathbf{d}_{\mathbf{F}}=\mathbf{A}_{\mathbf{F}} \mathbf{M}_{\mathbf{F}},
$$

where $\mathbf{d}_{\mathbf{F}}$ is a vector consisting of the hourly values of the Sq field,

$$
\mathbf{d}_{\mathbf{F}}=\left(\begin{array}{c}
\Delta V_{0} \\
\Delta V_{1} \\
\vdots \\
\Delta V_{23},
\end{array}\right)
$$

$\mathbf{A}_{\mathbf{F}}$ is a matrix of cosine and sine Fourier functions,

$$
\mathbf{A}_{\mathbf{F}}=\left(\begin{array}{cccccccc}
1 & \cos \left(2 \pi \frac{1 \cdot 0}{24}\right) & \cos \left(2 \pi \frac{2 \cdot 0}{24}\right) & \cos \left(2 \pi \frac{3 \cdot 0}{24}\right) & \cos \left(2 \pi \frac{4 \cdot 0}{24}\right) & \sin \left(2 \pi \frac{1 \cdot 0}{24}\right) & \ldots & \sin \left(2 \pi \frac{4 \cdot 0}{24}\right) \\
1 & \cos \left(2 \pi \frac{1 \cdot 1}{24}\right) & \cos \left(2 \pi \frac{2 \cdot 1}{24}\right) & \cos \left(2 \pi \frac{3 \cdot 1}{24}\right) & \cos \left(2 \pi \frac{4 \cdot 1}{24}\right) & \sin \left(2 \pi \frac{1 \cdot 1}{24}\right) & \ldots & \sin \left(2 \pi \frac{4 \cdot 1}{24}\right) \\
1 & \cos \left(2 \pi \frac{1 \cdot 2}{24}\right) & \cos \left(2 \pi \frac{2 \cdot 2}{24}\right) & \cos \left(2 \pi \frac{3 \cdot 2}{24}\right) & \cos \left(2 \pi \frac{4 \cdot 2}{24}\right) & \sin \left(2 \pi \frac{1 \cdot 2}{24}\right) & \ldots & \sin \left(2 \pi \frac{4 \cdot 2}{24}\right) \\
\vdots & \vdots & \vdots & \vdots & \vdots & \vdots & \ddots & \vdots \\
1 & \cos \left(2 \pi \frac{1 \cdot 23}{24}\right) & \cos \left(2 \pi \frac{2 \cdot 23}{24}\right) & \cos \left(2 \pi \frac{3 \cdot 23}{24}\right) & \cos \left(2 \pi \frac{4 \cdot 23}{24}\right) & \sin \left(2 \pi \frac{1 \cdot 23}{24}\right) & \ldots & \sin \left(2 \pi \frac{4 \cdot 23}{24}\right)
\end{array}\right),
$$


and $\mathbf{M}_{\mathbf{F}}$ is a vector of the Fourier coefficients:

$$
\mathbf{M}_{\mathbf{F}}=\left(\begin{array}{c}
a_{0} \\
a_{1} \\
a_{2} \\
a_{3} \\
a_{4} \\
b_{1} \\
\vdots \\
b_{4}
\end{array}\right) .
$$

Using measurements for $\mathbf{d}_{\mathbf{F}}$, (23) can be solved for $\mathbf{M}_{\mathbf{F}}$ as follows:

$$
\mathbf{M}_{\mathbf{F}}=\left\{\left(\mathbf{A}_{\mathbf{F}}\right)^{\mathrm{T}} \mathbf{A}_{\mathbf{F}}\right\}^{-1}\left\{\left(\mathbf{A}_{\mathbf{F}}\right)^{\mathrm{T}} \mathbf{d}_{\mathbf{F}}\right\} .
$$

It gives a mathematically compact expression of $\mathbf{S q}$, as $\mathbf{M}_{\mathbf{F}}$ consists of only nine values while the original data are composed of 24 hourly values. The method described above can be applied not only to the data for an individual day but also to the data for multiple days. In the latter case, the vector $\mathbf{d}_{\mathbf{F}}$ and matrix $\mathbf{A}_{\mathbf{F}}$ need to be extended in the row direction according to the number of data used. For example, if hourly data for five days are used, the length of the vector $\mathbf{d}_{\mathbf{F}}$ becomes $120(=24 \mathrm{~h} \times 5 \mathrm{~d})$, and the size of the matrix $\mathbf{A}_{\mathbf{F}}$ becomes $120 \times 9$.

The harmonic coefficients $a_{m}$ and $b_{m}$ vary temporally with season and solar activity, and spatially with latitude and longitude (e.g., Rastogi and Iyer 1976; Campbell 1982; Campbell et al. 1989, 1992; Yamazaki et al. 2011b). It is important to understand these variations because they can reveal the physical processes that govern the ionospheric dynamo. In Sect. 4 , we discuss in detail main characteristics of Sq and physical mechanisms behind them.

\subsection{Principal Component Analysis}

A principal component analysis also seeks for a mathematically compact representation of Sq. The principal component analysis resembles the Fourier analysis in that it expands Sq into series of orthogonal functions. However, unlike the Fourier analysis, the principal component analysis does not use a fixed set of basis functions. The advantage of the principal component analysis over the Fourier analysis is that it better reproduces the original data when the same number of basis functions are used, thus it is "more compact". The disadvantage of the principal component analysis is the difficulties in interpreting the physical meaning of each principal component.

The principal component analysis of $\mathrm{Sq}$ is generally conducted for a selected station during a certain period. Depending on the purpose of the analysis, the data length could be days, a month, a year, or longer. Assume that there are, in total, $N$ quiet days in the data set. For each of those quiet days, the Sq variation (in an arbitrary component) can be determined after the subtraction of the nighttime baseline and non-cyclic variation (see Sect. 3.2). It is possible to construct a $N \times 24$ matrix $\mathbf{Q}$ with its element $Q_{i j}$ representing Sq values at the $j$-th hour of the $i$-th day $(j=1,2, \ldots, 24$, and $i=1,2, \ldots, N)$. For the principal component analysis to work properly, we need to subtract the mean of each column from each element. The residual matrix $\mathbf{R}(N \times 24)$ is, thus,

$$
R_{i j}=Q_{i j}-\frac{1}{N} \sum_{k=1}^{N} Q_{k j} .
$$


The next step to derive the principal components is to calculate the covariance matrix of $\mathbf{R}$, which is,

$$
\mathbf{C}=\frac{1}{N} \mathbf{R}^{\mathrm{T}} \mathbf{R} .
$$

Here $\mathbf{R}^{\mathrm{T}}$ represents the transpose of the matrix $\mathbf{R}$. Since $\mathbf{C}$ is a square matrix $(24 \times 24)$, we can calculate the eigenvalues $\lambda_{m}$ and eigenvectors $\boldsymbol{\omega}_{m}(m=1,2, \ldots, 24)$ that satisfy

$$
\left(\mathbf{C}-\lambda_{m} \mathbf{I}\right) \boldsymbol{\omega}_{m}=0,
$$

where $\mathbf{I}$ is a $24 \times 24$ identity matrix. The eigenvectors are normalized so that the sum of the squares of the elements is one. Also, eigenvalues are sorted from the largest for $m=1$ to the smallest for $m=24$. In this way, $\omega_{m}$ becomes the $m$-th principal component of the data set. The multiplication of $\boldsymbol{\omega}_{m}$ on the left of the matrix $\mathbf{R}$ gives a vector $\mathbf{S}_{m}\left(=\mathbf{R} \boldsymbol{\omega}_{m}\right)$ of length $N$, whose $i$-th component represents the factor score of the $m$-th principal component on the day $i$. Using the factor score and principal components, the matrix $\mathbf{R}$ can be reconstructed as follows,

$$
\mathbf{R}=\sum_{m=1}^{24} \mathbf{S}_{m} \boldsymbol{\omega}_{m} .
$$

Usually, the first few principal components are sufficient to reproduce main features of the $\mathrm{Sq}$ variation. The original data set $\mathbf{Q}$ can be derived by adding $\mathbf{R}$ to the average $\mathrm{Sq}$ variation of the data set (see (28)).

Once the principal components are determined, one needs to interpret the physical significance of each component according to their behaviors. Obviously, the meaning of principal components could be different for different components of the geomagnetic field, for different stations, and for different selections of the data length. The following is a (partial) list of Sq studies in which the principal component analysis is used: Golovkov et al. (1978), Rajaram (1983), Alex et al. (1998), Gurubaran (2002), Yamada (2002, 2009), Xu and Kamide (2004), Stening et al. (2005a), Chen et al. (2007), Bhardwaj et al. (2015), Bhattacharyya and Okpala (2015). The first principal component (added to the average daily variation) is often interpreted as the contribution of normal Sq currents. Other principal components may represent currents with different sources that are superposed on the Sq currents.

\subsection{Equivalent Currents}

As mentioned in Sect. 1, it is not possible to know the three-dimensional ionospheric current system merely by analyzing Sq variations on the ground, but techniques allow us to determine the two-dimensional (horizontal) equivalent current system that produces exactly the same Sq variations as the actual ionospheric current system. The most simple way to evaluate equivalent currents is to assume a horizontal current sheet in the ionosphere. Figure 20 illustrates an infinite current sheet with the current intensity $I(\mathrm{~A} / \mathrm{m})$ flowing in the $\mathbf{y}$ direction (perpendicular to the plane of the paper, pointing outward). Let $P$ denote a point at a distance $h$ from the current sheet, assuming that $P$ is a ground magnetic observatory, and $I$ is the Sq current intensity flowing at an altitude $h$. The current sheet can be divided into a number of small portions flowing along strips with width $d x$. Each strip can be treated as an infinite line current with the strength of $I d x$. According to Biot-Savart law, the magnetic field at $P$ produced by the line current at $x$ is

$$
F=\frac{\mu_{0} I d x}{2 \pi \sqrt{h^{2}+x^{2}}} .
$$


Fig. 20 The magnetic field produced by an infinite current sheet

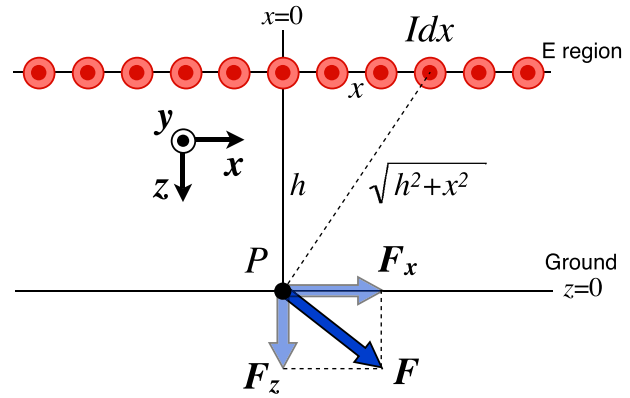

The components parallel to the $z$ and $x$ axes are

$$
\begin{aligned}
& F_{z}=\frac{\mu_{0} I d x}{2 \pi \sqrt{h^{2}+x^{2}}} \frac{x}{\sqrt{h^{2}+x^{2}}}, \\
& F_{x}=\frac{\mu_{0} I d x}{2 \pi \sqrt{h^{2}+x^{2}}} \frac{h}{\sqrt{h^{2}+x^{2}}} .
\end{aligned}
$$

At the same time, the line current at $-x$ produce the magnetic fields $-F_{z}$ and $F_{x}$ in the $z$ and $x$ directions, respectively. Thus, the total magnetic field produced by line currents at distances $\pm x$ is parallel to the $x$ axis and the magnitude is $2 F_{x}$. The integration of $2 F_{x}$ (see (34)) from $x=0$ to $x=\infty$ gives the net contribution by the current sheet:

$$
\Delta B=\int_{0}^{\infty} 2 F_{x} d x=\frac{\mu_{0} h I}{\pi} \int_{0}^{\infty} \frac{d x}{\sqrt{h^{2}+x^{2}}}=\frac{\mu_{0} I}{2},
$$

where $\Delta B$ in units of tesla. Equation (35) can be rewritten in more commonly used units of nano tesla:

$$
\Delta b=\frac{2 \pi}{10} J
$$

where $\Delta b$ is the magnetic field in $\mathrm{nT}$ and $J$ is the current density in $\mathrm{mA} / \mathrm{m}$. One can also derive (36) from Ampère's Law applied to an infinite current sheet. The results suggest that the magnitude of the magnetic field by a current sheet is proportional to the current density but independent of the distance from the current sheet. The direction of the magnetic field is the direction of the current rotated by $90^{\circ}$ counterclockwise when viewed from above.

The ground-level magnetic perturbation results not only from ionospheric currents but also from Earth currents induced by ionospheric currents. For the horizontal component of Sq variations, the contribution of Earth currents account for approximately one-third. Taking this effect into consideration, Sq variations in the northward $(X)$ and eastward $(Y)$ component can be approximated as follows:

$$
\begin{gathered}
J_{E}=\frac{2}{3} \frac{10}{2 \pi} \Delta X \sim \Delta X, \\
J_{N}=-\frac{2}{3} \frac{10}{2 \pi} \Delta Y \sim-\Delta Y,
\end{gathered}
$$

where $J_{E}$ and $J_{N}$ are eastward and northward current densities in $\mathrm{mA} / \mathrm{m}$, respectively. It is useful to remember that a horizontal magnetic perturbation of $1 \mathrm{nT}$ roughly corresponds to an ionospheric current of $1 \mathrm{~mA} / \mathrm{m}$. 


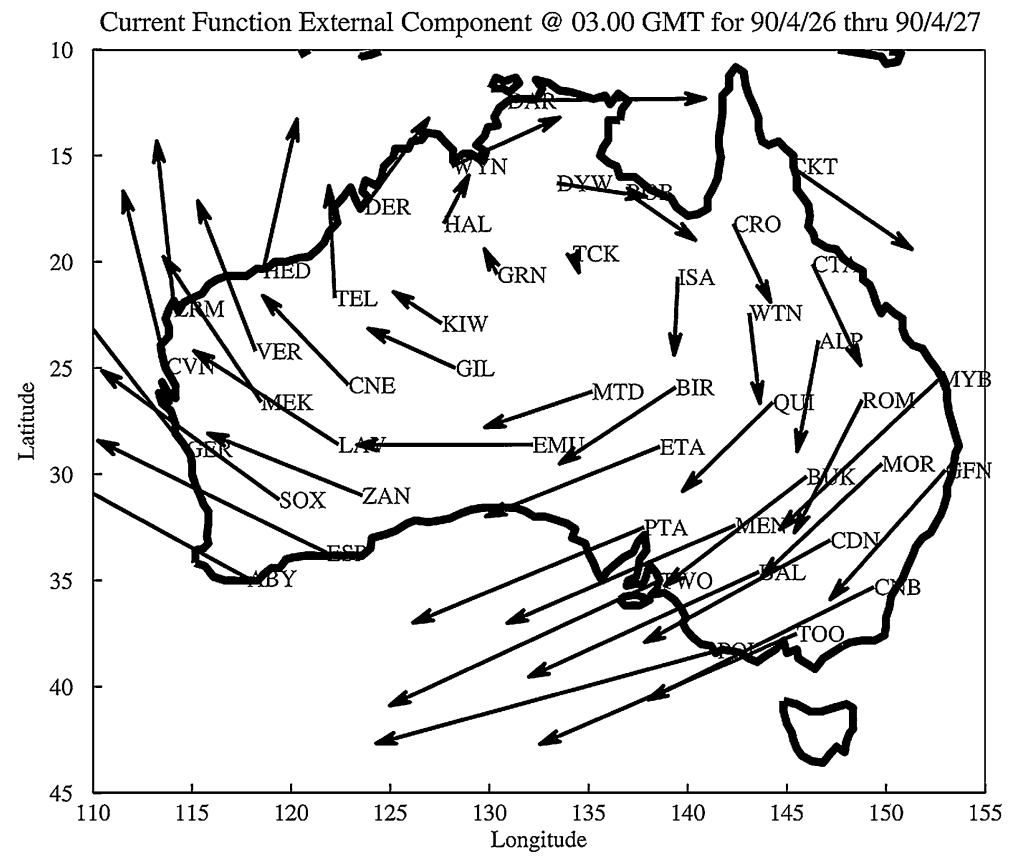

Fig. 21 A map of equivalent Sq current vectors at 0300 UT on 27 April 1990. An arrow having the length of $5^{\circ}$ longitude corresponds to an ionospheric current of approximately $31 \mathrm{~mA} / \mathrm{m}$. From Stening et al. (2005a)

Figure 21 is an example of the equivalent Sq current system derived using the method described above (after Stening et al. 2005a). The extensive measurements by AWAGS (Australia-Wide Array of Geomagnetic Stations; Chamalaun and Barton 1993) clearly reveal the clockwise vortex of the southern-hemisphere Sq current system over Australia. In the figure, an arrow length of $5^{\circ}$ longitude corresponds to a magnetic perturbation of $31 \mathrm{nT}$, and thus $\sim 31 \mathrm{~mA} / \mathrm{m}$.

As can be seen from the fact that $\mathrm{Sq}$ variations exist in the $\mathrm{Z}$ component as well as in the horizontal component, the current-sheet assumption of Sq currents is valid only to a limited extent. In the following section, we introduce a spherical harmonic analysis technique, which makes it possible to derive equivalent currents without the current-sheet assumption.

\subsection{Spherical Harmonic Analysis}

Spherical harmonic analysis is a powerful technique to describe the global distribution of the geomagnetic field including, but not limited to, the Sq field. The Sq field at a certain location can be measured using a magnetometer. However, ground-based measurements can cover only a limited area of the surface due to, for example, the presence of oceans. Thus, at the locations where there are no measurements, the Sq field needs to be estimated by an interpolation of existing data. Such an interpolation should be based on mathematical equations that are physically meaningful. Spherical harmonic analysis uses harmonic functions that satisfy Laplace's equation in spherical coordinates. Observational data are used to determine a set of harmonic functions, or a "model", in such a manner as to minimize the difference between the model and observations. The idea is very similar to the Fourier analysis described in Sect. 3.3, but the spherical harmonic analysis uses a 2-D model (a function 
of latitude and longitude) while the Fourier analysis uses a 1-D model (a function of time). The mathematical basis for the spherical harmonic analysis was developed by Legendre and Laplace for the description of gravitational potential. The spherical harmonic analysis was introduced to geomagnetism by Gauss (1841), and the first application of the technique to the Sq field was made by Schuster $(1889,1908)$. One of the benefits of the spherical harmonic analysis is that it enables the separation of external and internal fields, which led early scientists to discover that Sq variations have their origin outside the Earth's surface. The spherical harmonic analysis also enables to calculate equivalent currents for Sq, without the assumption of an infinite current sheet that was detailed in Sect. 3.6.

Several methods exist for the spherical harmonic analysis of the Sq field. We describe two methods in this paper, namely, the "instantaneous method" and the "slice method". The instantaneous method determines the Sq field and equivalent current system at a specific moment in time using simultaneous measurements from globally distributed magnetometers (e.g., Malin 1973; Parkinson 1977; Suzuki 1978, 1979; Takeda and Araki 1984a; Takeda 1999, 2002a, 2002b). This method is useful when a large number of magnetic data are available from all over the world. On the other hand, the slice method uses only a northsouth chain of observatories and assumes that the 24 hours of Sq field variation represents the $360^{\circ}$ longitudinal variation (Matsushita and Maeda 1965a; Suzuki 1973; Campbell and Matsushita 1982; Campbell and Schiffmacher 1985, 1988b; Kawasaki et al. 1989; Yamazaki et al. 2010, 2011a). As the Earth rotates, the chain of magnetometers travels under the Sq current system that is fixed in pattern on the dayside of the Earth. The magnetometer array, thus, completes a $360^{\circ}$ longitudinal scan of the global Sq field in 24 hours time. The slice method is useful when magnetic data are available only from a particular longitude. The time resolution of the results cannot be better than a day as it takes 24 hours to obtain the complete Sq field by a single magnetometer array. The comparison between the instantaneous method and slice method was made by Campbell (1990), revealing generally good agreement.

In both methods, or in spherical harmonic analysis in general, a mathematical model comes from steady-state Maxwell's equations for the magnetic field $\mathbf{B}$,

$$
\begin{gathered}
\nabla \cdot \mathbf{B}=0, \\
\nabla \times \mathbf{B}=\mu_{0} \mathbf{J},
\end{gathered}
$$

where $\mathbf{J}$ denotes the electric current density and $\mu_{0}$ is the magnetic permeability of free space. Under the assumption that the region under consideration is free of magnetic field sources,

$$
\nabla \times \mathbf{B}=0
$$

Thus, it is possible to express $\mathbf{B}$ using a magnetic potential $M$,

$$
\mathbf{B}=-\nabla M
$$

The source free assumption is, in general, valid between the Earth's surface and ionosphere as there is little current flowing in the atmosphere below the ionosphere. Thus, the assumption holds for the Sq field measured on the ground, but at satellite altitudes in the ionosphere (200-600 km), the effect of inter-hemispheric field-aligned currents may need to be taken into account (see Pedatella et al. 2011 for more discussion). From (39) and (42)

$$
\nabla^{2} M=0
$$


which is commonly known as Laplace's equation. In spherical coordinates with the origin at the center of the Earth, (43) becomes

$$
\frac{\partial}{\partial r}\left(r^{2} \frac{\partial M}{\partial r}\right)+\frac{1}{\sin \theta} \frac{\partial}{\partial \theta}\left(\sin \theta \frac{\partial M}{\partial \theta}\right)+\frac{1}{\sin ^{2} \theta} \frac{\partial^{2} M}{\partial \phi^{2}}=0
$$

where $r, \theta, \phi$ are the radial distance, colatitude, and longitude, respectively. The magnetic potential relates to the northward, eastward, and vertical components of the magnetic field as follows:

$$
\begin{gathered}
X=\frac{1}{r} \frac{\partial M}{\partial \theta}, \\
Y=-\frac{1}{r \sin \theta} \frac{\partial M}{\partial \phi}, \\
Z=\frac{\partial M}{\partial r} .
\end{gathered}
$$

The general solution for (44) can be written in the following form (e.g., Riley et al. 2002, pp. 664-667):

$$
\begin{gathered}
M(r, \theta, \phi)=C+M_{\mathrm{ex}}(r, \theta, \phi)+M_{\mathrm{in}}(r, \theta, \phi), \\
M_{\mathrm{ex}}=R_{E} \sum_{n=1}^{\infty}\left[\left(\frac{r}{R_{E}}\right)^{n} \sum_{m=0}^{n}\left\{g_{\mathrm{ex}_{n}}^{m} \cos (m \phi)+h_{\mathrm{ex}_{n}}^{m} \sin (m \phi)\right\} P_{n}^{m}(\cos \theta)\right], \\
M_{\mathrm{in}}=R_{E} \sum_{n=1}^{\infty}\left[\left(\frac{R_{E}}{r}\right)^{n+1} \sum_{m=0}^{n}\left\{g_{\mathrm{in}_{n}}^{m} \cos (m \phi)+h_{\mathrm{in}_{n}}^{m} \sin (m \phi)\right\} P_{n}^{m}(\cos \theta)\right],
\end{gathered}
$$

where $C$ is an arbitrary constant and $R_{E}$ is the Earth's radius. The Earth is assumed to be a perfect sphere. The consideration of the deviation of the Earth's surface from a sphere makes little difference in the results of the spherical harmonic analysis (Chapman and Bartels 1940, p. 640). The two components of the magnetic potential, $M_{\mathrm{ex}}$ and $M_{\mathrm{in}}$, are generally attributed to external and internal sources, respectively. This is because $M_{\mathrm{ex}}$ increases with increasing $r$ (i.e., $M_{\mathrm{ex}}$ increases with the height from the surface) while $M_{\text {in }}$ increases with decreasing $r$ (i.e., $M_{\text {in }}$ increases with the depth from the ground). In (47) and (48), $n$ and $m$ are the harmonic degree and order, respectively. $g_{n}^{m}$ and $h_{n}^{m}$ are the so-called Gauss coefficients, with subscripts "ex" and "in" representing external and internal components, respectively. It can be seen in (47) and (48) that the longitudinal dependence of $M$ is expressed by Fourier series. On the other hand, the latitudinal dependence of $M$ is represented by a series of Schmidt quasi-normalized associated Legendre functions $P_{n}^{m}(\cos \theta) . P_{n}^{m}(\cos \theta)$ can be calculated using the following recursion formula (Campbell 2003, p. 21):

$$
\begin{aligned}
& P_{n}^{m}(\cos \theta)= \begin{cases}\sqrt{\frac{2 m-1}{2 m}} \sin (\theta) P_{n-1}^{m-1}(\cos \theta) & (m>1, n=m), \\
\frac{(2 n-1) \cos (\theta) P_{n-1}^{m}(\cos \theta)-R_{n-1}^{m} P_{n-2}^{m}(\cos \theta)}{R_{n}^{m}} & (n>m),\end{cases} \\
& R_{n}^{m}=\sqrt{n^{2}-m^{2}} \\
& P_{0}^{0}(\cos \theta)=1, \quad P_{1}^{0}(\cos \theta)=\cos (\theta), \quad P_{1}^{1}(\cos \theta)=\sin (\theta),
\end{aligned}
$$


and for later use,

$$
\frac{d P_{n}^{m}(\cos \theta)}{d \theta}=\frac{n \cos (\theta) P_{n}^{m}(\cos \theta)-R_{n}^{m} P_{n-1}^{m}(\cos \theta)}{\sin \theta} \quad\left(0^{\circ}<\theta<180^{\circ}\right) .
$$

The functions $\left\{g_{n}^{m} \cos (m \phi)+h_{n}^{m} \sin (m \phi)\right\} P_{n}^{m}(\cos \theta)$ that appear in (47) and (48) represent wave modes on a spherical surface. Different combinations of $(n, m)$ provide different wave modes that are orthogonal to each other. The Gauss coefficients determine the amplitude and phase of each mode. Figure 22 displays the wave pattern for some selected modes. Each mode has $m$ waves at a fixed latitude and $(n-m+1) / 2$ waves between the North and South Poles at a fixed longitude.

We will now describe how to determine the external and internal magnetic potentials, $M_{\text {ex }}$ and $M_{\text {in }}$, using Sq data. The instantaneous method requires Sq variations in the $X, Y$ and $Z$ components at a number of locations world-wide for a certain day. At a given time of the day, let Sq at a particular observatory be denoted by $\Delta X_{i}, \Delta Y_{i}$, and $\Delta Z_{i}$, where $i$ $(=1,2,3, \ldots, k)$ corresponds to each observatory at different locations $\left(\theta_{i}, \phi_{i}\right) . k$ denotes the total number of the observatories involved. Using (45)-(48), we can evaluate magnetic perturbations at the surface of the Earth $\left(r=R_{E}\right)$ for $\Delta X_{i}, \Delta Y_{i}$, and $\Delta Z_{i}$. That is,

$$
\begin{aligned}
\Delta X_{i}= & \sum_{n=1}^{N} \sum_{m=0}^{n}\left\{\frac{d P_{n}^{m}\left(\cos \theta_{i}\right)}{d \theta} \cos \left(m \phi_{i}\right)\right\}\left(g_{\mathrm{ex}_{n}^{m}}^{m}+g_{\mathrm{in}_{n}}^{m}\right) \\
& +\sum_{n=1}^{N} \sum_{m=0}^{n}\left\{\frac{d P_{n}^{m}\left(\cos \theta_{i}\right)}{d \theta} \sin \left(m \phi_{i}\right)\right\}\left(h_{\mathrm{ex}_{n}^{m}}^{m}+h_{\mathrm{in}_{n}}^{m}\right), \\
\Delta Y_{i}= & \sum_{n=1}^{N} \sum_{m=0}^{n}\left\{m P_{n}^{m}\left(\cos \theta_{i}\right) \sin \left(m \phi_{i}\right)\right\}\left(g_{\mathrm{ex}_{n}}^{m}+g_{\mathrm{in}_{n}}^{m}\right) \\
& -\sum_{n=1}^{N} \sum_{m=0}^{n}\left\{m P_{n}^{m}\left(\cos \theta_{i}\right) \cos \left(m \phi_{i}\right)\right\}\left(h_{\mathrm{ex}_{n}}^{m}+h_{\mathrm{in}_{n}}^{m}\right), \\
\Delta Z_{i}= & \sum_{n=1}^{N} \sum_{m=0}^{n}\left\{P_{n}^{m}\left(\cos \theta_{i}\right) \cos \left(m \phi_{i}\right)\right\}\left(n g_{\operatorname{ex}_{n}}^{m}-(n+1) g_{\operatorname{in}_{n}}^{m}\right) \\
+ & \sum_{n=1}^{N} \sum_{m=0}^{n}\left\{P_{n}^{m}\left(\cos \theta_{i}\right) \sin \left(m \phi_{i}\right)\right\}\left(n h_{\operatorname{ex}_{n}^{m}}^{m}-(n+1) h_{\mathrm{in}_{n}}^{m}\right) .
\end{aligned}
$$

The harmonic series needs to be truncated after a finite number $n=N$. The truncation level is typically chosen to be $n=4-8$ for the instantaneous method, ignoring the equatorial and auroral electrojets, which require much larger $n$. For a given combination of $(n, m)$, all the terms in the curly brackets are known from (49)-(52). Therefore, in principle, $\left(g_{\mathrm{ex}_{n}}{ }_{n}+\right.$ $\left.g_{\text {in }}^{m}\right)$ and $\left(h_{\operatorname{ex}_{n}}^{m}+h_{\text {in }_{n}}^{m}\right)$ can be determined from $\Delta X$ and/or $\Delta Y$. Meanwhile, $\left(n g_{\operatorname{ex}_{n}}^{m}-\right.$ $\left.(n+1) g_{\text {in }_{n}^{m}}^{m}\right)$ and $\left(n h_{\mathrm{ex}_{n}^{m}}^{m}-(n+1) h_{\mathrm{in}_{n}}^{m}\right)$ can be determined from $\Delta Z$. Thus, combining the measurements in the horizontal and vertical components, it is possible to derive all the Gauss coefficients $g_{\operatorname{ex}_{n}^{m}}^{m}, g_{\mathrm{in}_{n}}^{m}, h_{\mathrm{ex}_{n}}^{m}$, and $h_{\mathrm{in}_{n}}^{m}$. In order to avoid lengthy formulae, we define $a_{n}^{m}, b_{n}^{m}$, $A_{n}^{m}$, and $B_{n}^{m}$ as follows:

$$
\begin{array}{cl}
a_{n}^{m}=g_{\operatorname{ex}_{n}^{m}}^{m}+g_{\operatorname{in}_{n}}^{m}, & b_{n}^{m}=h_{\operatorname{ex}_{n}^{m}}^{m}+h_{\operatorname{in}_{n}}^{m}, \\
A_{n}^{m}=n g_{\operatorname{ex}_{n}^{m}}^{m}-(n+1) g_{\operatorname{in}_{n}^{m}}^{m}, & B_{n}^{m}=n h_{\operatorname{ex}_{n}^{m}}^{m}-(n+1) h_{\mathrm{in}_{n}}^{m},
\end{array}
$$



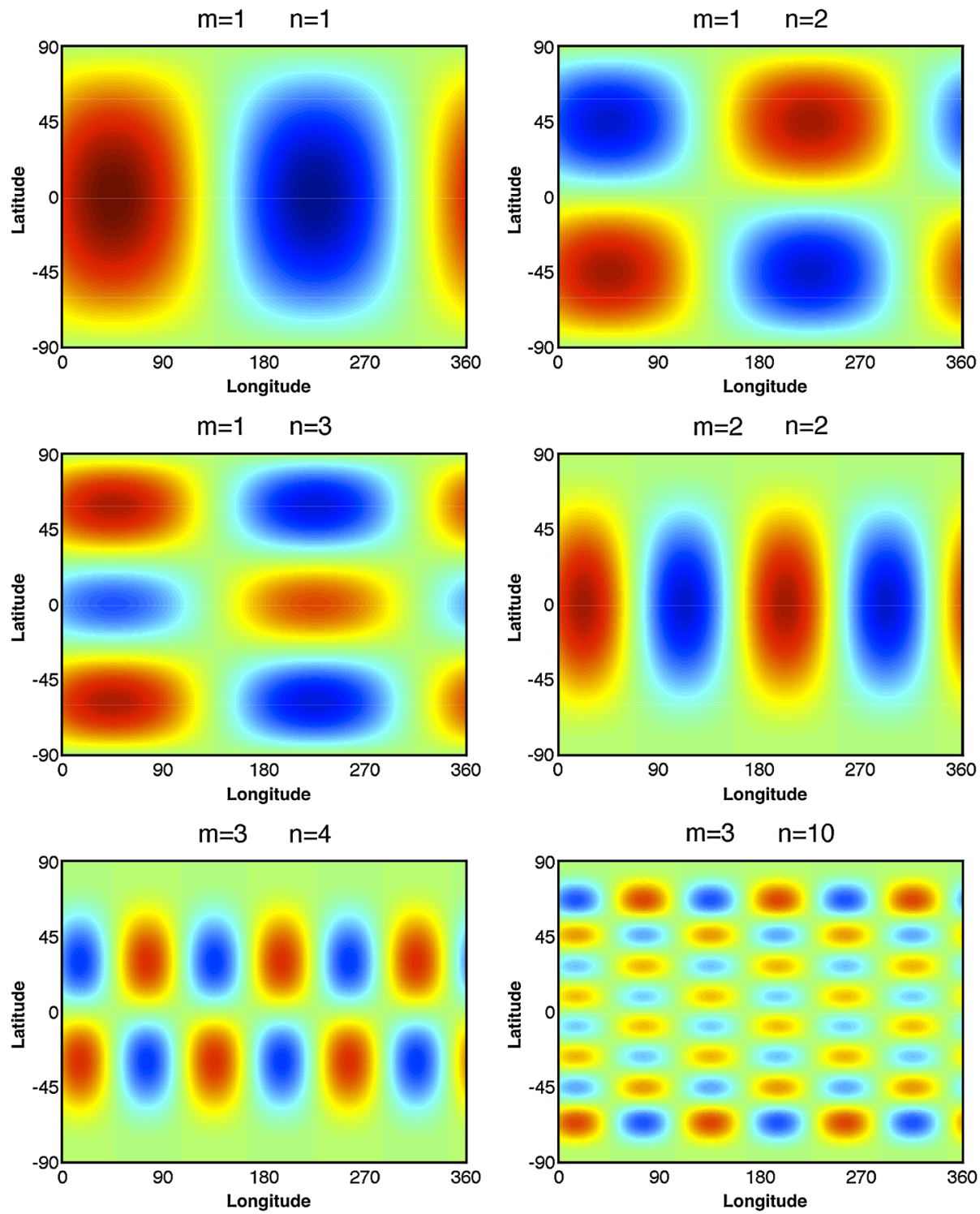

Fig. 22 Examples of spherical harmonic functions

and $\hat{X}_{n}^{m}, \check{X}_{n}^{m}, \hat{Y}_{n}^{m}, \check{Y}_{n}^{m}, \hat{Z}_{n}^{m}$, and $\check{Z}_{n}^{m}$ as follows:

$$
\begin{array}{cc}
\hat{X}(\theta, \phi)_{n}^{m}=\frac{d P_{n}^{m}(\cos \theta)}{d \theta} \cos (m \phi), & \check{X}(\theta, \phi)_{n}^{m}=\frac{d P_{n}^{m}(\cos \theta)}{d \theta} \sin (m \phi), \\
\hat{Y}(\theta, \phi)_{n}^{m}=m P_{n}^{m}(\cos \theta) \sin (m \phi), & \check{Y}(\theta, \phi)_{n}^{m}=-m P_{n}^{m}(\cos \theta) \cos (m \phi), \\
\hat{Z}(\theta, \phi)_{n}^{m}=P_{n}^{m}(\cos \theta) \cos (m \phi), & \check{Z}(\theta, \phi)_{n}^{m}=P_{n}^{m}(\cos \theta) \sin (m \phi) .
\end{array}
$$


Now (53)-(54) can be reduced to a matrix equation:

$$
\mathbf{d}_{1}=\mathbf{A}_{1} \mathbf{M}_{1},
$$

where $\mathbf{d}_{\mathbf{1}}$ is a vector for $\mathrm{Sq}$ in the $X$ and $Y$ components,

$$
\mathbf{d}_{\mathbf{1}}=\left(\begin{array}{c}
\Delta X_{1} \\
\Delta X_{2} \\
\vdots \\
\Delta X_{k} \\
\Delta Y_{1} \\
\Delta Y_{2} \\
\vdots \\
\Delta Y_{k}
\end{array}\right),
$$

$\mathbf{A}_{1}$ is a matrix of spherical harmonic functions,

$$
\mathbf{A}_{\mathbf{1}}=\left(\begin{array}{cccccccc}
\hat{X}\left(\theta_{1}, \phi_{1}\right)_{0}^{1} & \hat{X}\left(\theta_{1}, \phi_{1}\right)_{1}^{1} & \hat{X}\left(\theta_{1}, \phi_{1}\right)_{0}^{2} & \ldots & \hat{X}\left(\theta_{1}, \phi_{1}\right)_{N}^{N} & \check{X}\left(\theta_{1}, \phi_{1}\right)_{0}^{1} & \ldots & \check{X}\left(\theta_{1}, \phi_{1}\right)_{N}^{N} \\
\hat{X}\left(\theta_{2}, \phi_{2}\right)_{0}^{1} & \hat{X}\left(\theta_{2}, \phi_{2}\right)_{1}^{1} & \hat{X}\left(\theta_{2}, \phi_{2}\right)_{0}^{2} & \ldots & \hat{X}\left(\theta_{2}, \phi_{2}\right)_{N}^{N} & \check{X}\left(\theta_{2}, \phi_{2}\right)_{0}^{1} & \ldots & \check{X}\left(\theta_{2}, \phi_{2}\right)_{N}^{N} \\
\hat{X}\left(\theta_{3}, \phi_{3}\right)_{0}^{1} & \hat{X}\left(\theta_{3}, \phi_{3}\right)_{1}^{1} & \hat{X}\left(\theta_{3}, \phi_{3}\right)_{0}^{2} & \ldots & \hat{X}\left(\theta_{3}, \phi_{3}\right)_{N}^{N} & \check{X}\left(\theta_{3}, \phi_{3}\right)_{0}^{1} & \ldots & \check{X}\left(\theta_{3}, \phi_{3}\right)_{N}^{N} \\
\vdots & \vdots & \vdots & \vdots & \vdots & \vdots & \vdots & \vdots \\
\hat{X}\left(\theta_{k}, \phi_{k}\right)_{0}^{1} & \hat{X}\left(\theta_{k}, \phi_{k}\right)_{1}^{1} & \hat{X}\left(\theta_{k}, \phi_{k}\right)_{0}^{2} & \ldots & \hat{X}\left(\theta_{k}, \phi_{k}\right)_{N}^{N} & \check{X}\left(\theta_{k}, \phi_{k}\right)_{0}^{1} & \ldots & \check{X}\left(\theta_{k}, \phi_{k}\right)_{N}^{N} \\
\hat{Y}\left(\theta_{1}, \phi_{1}\right)_{0}^{1} & \hat{Y}\left(\theta_{1}, \phi_{1}\right)_{1}^{1} & \hat{Y}\left(\theta_{1}, \phi_{1}\right)_{0}^{2} & \ldots & \hat{Y}\left(\theta_{1}, \phi_{1}\right)_{N}^{N} & \check{Y}\left(\theta_{1}, \phi_{1}\right)_{0}^{1} & \ldots & \check{Y}\left(\theta_{1}, \phi_{1}\right)_{N}^{N} \\
\hat{Y}\left(\theta_{2}, \phi_{2}\right)_{0}^{1} & \hat{Y}\left(\theta_{2}, \phi_{2}\right)_{1}^{1} & \hat{Y}\left(\theta_{2}, \phi_{2}\right)_{0}^{2} & \ldots & \hat{Y}\left(\theta_{2}, \phi_{2}\right)_{N}^{N} & \check{Y}\left(\theta_{2}, \phi_{2}\right)_{0}^{1} & \ldots & \check{Y}\left(\theta_{2}, \phi_{2}\right)_{N}^{N} \\
\hat{Y}\left(\theta_{3}, \phi_{3}\right)_{0}^{1} & \hat{Y}\left(\theta_{3}, \phi_{3}\right)_{1}^{1} & \hat{Y}\left(\theta_{3}, \phi_{3}\right)_{0}^{2} & \ldots & \hat{Y}\left(\theta_{3}, \phi_{3}\right)_{N}^{N} & \check{Y}\left(\theta_{3}, \phi_{3}\right)_{0}^{1} & \ldots & \check{Y}\left(\theta_{3}, \phi_{3}\right)_{N}^{N} \\
\vdots & \vdots & \vdots & \vdots & \vdots & \vdots & \vdots & \vdots \\
\hat{Y}\left(\theta_{k}, \phi_{k}\right)_{0}^{1} & \hat{Y}\left(\theta_{k}, \phi_{k}\right)_{1}^{1} & \hat{Y}\left(\theta_{k}, \phi_{k}\right)_{0}^{2} & \ldots & \hat{Y}\left(\theta_{k}, \phi_{k}\right)_{N}^{N} & \check{Y}\left(\theta_{k}, \phi_{k}\right)_{0}^{1} & \ldots & \check{Y}\left(\theta_{k}, \phi_{k}\right)_{N}^{N}
\end{array}\right),
$$

and $\mathbf{M}_{\mathbf{1}}$ is a vector for the coefficients,

$$
\mathbf{M}_{1}=\left(\begin{array}{c}
a_{0}^{1} \\
a_{1}^{1} \\
a_{0}^{2} \\
\vdots \\
a_{N}^{N} \\
b_{0}^{1} \\
b_{1}^{1} \\
b_{0}^{2} \\
\vdots \\
b_{N}^{N}
\end{array}\right)
$$


Similar to the Fourier analysis described in Sect. 3.4, the least-squares solution for $\mathbf{M}_{\mathbf{1}}$ can be found as follows:

$$
\mathbf{M}_{\mathbf{1}}=\left\{\left(\mathbf{A}_{\mathbf{1}}\right)^{\mathrm{T}} \mathbf{A}_{\mathbf{1}}\right\}^{-1}\left\{\left(\mathbf{A}_{\mathbf{1}}\right)^{\mathrm{T}} \mathbf{d}_{\mathbf{1}}\right\}
$$

Meanwhile, equation (55) can also be written in a matrix form:

$$
\mathbf{d}_{2}=\mathbf{A}_{2} \mathbf{M}_{2}
$$

where $\mathbf{d}_{\mathbf{2}}$ is a vector for the vertical component of $\mathrm{Sq}$,

$$
\mathbf{d}_{2}=\left(\begin{array}{c}
\Delta Z_{1} \\
\Delta Z_{2} \\
\vdots \\
\Delta Z_{k}
\end{array}\right)
$$

$\mathbf{A}_{2}$ is a matrix of spherical harmonic functions,

$$
\mathbf{A}_{\mathbf{2}}=\left(\begin{array}{cccccccc}
\hat{Z}\left(\theta_{1}, \phi_{1}\right)_{0}^{1} & \hat{Z}\left(\theta_{1}, \phi_{1}\right)_{1}^{1} & \hat{Z}\left(\theta_{1}, \phi_{1}\right)_{0}^{2} & \ldots & \hat{Z}\left(\theta_{1}, \phi_{1}\right)_{N}^{N} & \check{Z}\left(\theta_{1}, \phi_{1}\right)_{0}^{1} & \ldots & \check{Z}\left(\theta_{1}, \phi_{1}\right)_{N}^{N} \\
\hat{Z}\left(\theta_{2}, \phi_{2}\right)_{0}^{1} & \hat{Z}\left(\theta_{2}, \phi_{2}\right)_{1}^{1} & \hat{Z}\left(\theta_{2}, \phi_{2}\right)_{0}^{2} & \ldots & \hat{Z}\left(\theta_{2}, \phi_{2}\right)_{N}^{N} & \check{Z}\left(\theta_{2}, \phi_{2}\right)_{0}^{1} & \ldots & \check{Z}\left(\theta_{2}, \phi_{2}\right)_{N}^{N} \\
\hat{Z}\left(\theta_{3}, \phi_{3}\right)_{0}^{1} & \hat{Z}\left(\theta_{3}, \phi_{3}\right)_{1}^{1} & \hat{Z}\left(\theta_{3}, \phi_{3}\right)_{0}^{2} & \ldots & \hat{Z}\left(\theta_{3}, \phi_{3}\right)_{N}^{N} & \check{Z}\left(\theta_{3}, \phi_{3}\right)_{0}^{1} & \ldots & \check{Z}\left(\theta_{3}, \phi_{3}\right)_{N}^{N} \\
\vdots & \vdots & \vdots & \vdots & \vdots & \vdots & \vdots & \vdots \\
\hat{Z}\left(\theta_{k}, \phi_{k}\right)_{0}^{1} & \hat{Z}\left(\theta_{k}, \phi_{k}\right)_{1}^{1} & \hat{Z}\left(\theta_{k}, \phi_{k}\right)_{0}^{2} & \ldots & \hat{Z}\left(\theta_{k}, \phi_{k}\right)_{N}^{N} & \check{Z}\left(\theta_{k}, \phi_{k}\right)_{0}^{1} & \ldots & \check{Z}\left(\theta_{k}, \phi_{k}\right)_{N}^{N}
\end{array}\right),
$$

and $\mathbf{M}_{\mathbf{2}}$ is a coefficient vector:

$$
\mathbf{M}_{2}=\left(\begin{array}{c}
A_{0}^{1} \\
A_{1}^{1} \\
A_{0}^{2} \\
\vdots \\
A_{N}^{N} \\
B_{0}^{1} \\
B_{1}^{1} \\
B_{0}^{2} \\
\vdots \\
B_{N}^{N}
\end{array}\right) .
$$

The least-squares solution for $\mathbf{M}_{\mathbf{2}}$ is

$$
\mathbf{M}_{\mathbf{2}}=\left\{\left(\mathbf{A}_{\mathbf{2}}\right)^{\mathrm{T}} \mathbf{A}_{\mathbf{2}}\right\}^{-1}\left\{\left(\mathbf{A}_{\mathbf{2}}\right)^{\mathrm{T}} \mathbf{d}_{\mathbf{2}}\right\} .
$$


Once both $\mathbf{M}_{1}$ and $\mathbf{M}_{2}$ are calculated, the Gauss coefficients can be derived using (56), which can also be written as

$$
\begin{array}{ll}
g_{\operatorname{ex}_{n}^{m}}^{m}=\frac{(n+1) a_{n}^{m}+A_{n}^{m}}{2 n+1}, & g_{\text {in }_{n}}^{m}=\frac{n a_{n}^{m}-A_{n}^{m}}{2 n+1}, \\
h_{\operatorname{ex}_{n}^{m}}^{m}=\frac{(n+1) b_{n}^{m}+B_{n}^{m}}{2 n+1}, & h_{\text {in }_{n}}^{m}=\frac{n b_{n}^{m}-B_{n}^{m}}{2 n+1} .
\end{array}
$$

The Gauss coefficients can be substituted into (47) and (48) to determine the external and internal magnetic potentials, $M_{\mathrm{ex}}$ and $M_{\mathrm{in}}$. Also, the external and internal parts of the equivalent current function can be derived using the following formula (Chapman and Bartels 1940, p. 631):

$$
\begin{aligned}
J_{\mathrm{ex}} & =\sum_{n=1}^{\infty}\left[-\frac{10}{4 \pi} \frac{2 n+1}{n+1}\left(\frac{r}{R_{E}}\right)^{n} \sum_{m=0}^{n}\left\{g_{\mathrm{ex} n}^{m} \cos (m \phi)+h_{\mathrm{ex} n}^{m} \sin (m \phi)\right\} P_{n}^{m}(\cos \theta)\right], \\
J_{\mathrm{in}} & =\sum_{n=1}^{\infty}\left[\frac{10}{4 \pi} \frac{2 n+1}{n}\left(\frac{R_{E}}{r}\right)^{n+1} \sum_{m=0}^{n}\left\{g_{\mathrm{in} n}^{m} \cos (m \phi)+h_{\mathrm{in}_{n}}^{m} \sin (m \phi)\right\} P_{n}^{m}(\cos \theta)\right] .
\end{aligned}
$$

These current functions are the scalar potentials, in units of amperes, associated with the equivalent currents. The external component, $J_{\mathrm{ex}}$, represents an equivalent ionospheric $\mathrm{Sq}$ current system, and is typically evaluated at an altitude of $110 \mathrm{~km}$. The internal component, $J_{\text {in }}$, is generally smaller than the external counterpart, and is considered to be a secondary current system induced within the conducting Earth at a depth of $\sim 400 \mathrm{~km}$, as well as in the ocean $\sim 0 \mathrm{~km}$. Takeda (1985) reported that the intensity of the internal Sq currents increases when the external current system is over the ocean. Figure 3 shows examples of the external current function derived by the instantaneous method (after Takeda 2002a). The latitudinal and longitudinal components of the equivalent current intensity $I_{\theta}$ and $I_{\phi}$ can be computed from

$$
\begin{gathered}
I_{\theta}=\frac{1}{r \sin \theta} \frac{\partial J}{\partial \phi}, \\
I_{\phi}=-\frac{1}{r} \frac{\partial J}{\partial \theta}
\end{gathered}
$$

for both $J_{\text {ex }}$ and $J_{\text {in }}$.

In the slice method, Sq data are obtained from a north-south chain of magnetometers such as that shown in Fig. 2 (left). As mentioned earlier, the slice method assumes that a 24hour $\mathrm{Sq}$ variation represents the $360^{\circ}$ longitudinal variation. The validity of this assumption was studied by Patil and Rajaram (2001) for the Indian sector. Their results suggested that the Sq current system is stationary and the time dependence can be used to represent the longitudinal variations. Mathematical formulae for the slice method can be readily obtained by replacing longitude $\phi$ by local-time hour angle $\phi_{L T}$ in all the equations used for the instantaneous method $\left(\phi_{L T}=0^{\circ}\right.$ at $0000 \mathrm{LT}, \phi_{L T}=180^{\circ}$ at $1200 \mathrm{LT}$, and so on). In the slice method, observed Sq variations may be first smoothed and extrapolated in the meridional direction so that the Sq field will be defined at any location on evenly distributed latitude and local-time grids. Early studies used hand-drawing curves for smoothing (e.g., Matsushita and Maeda 1965a). Campbell (1982) and Yamazaki (2014) used five-point smoothing. Figure 23 (top) shows examples of the smoothed Sq field for the East Asian longitude sector (Fig. 2, left). The relatively small structures associated with EEJ are clearly visible in 

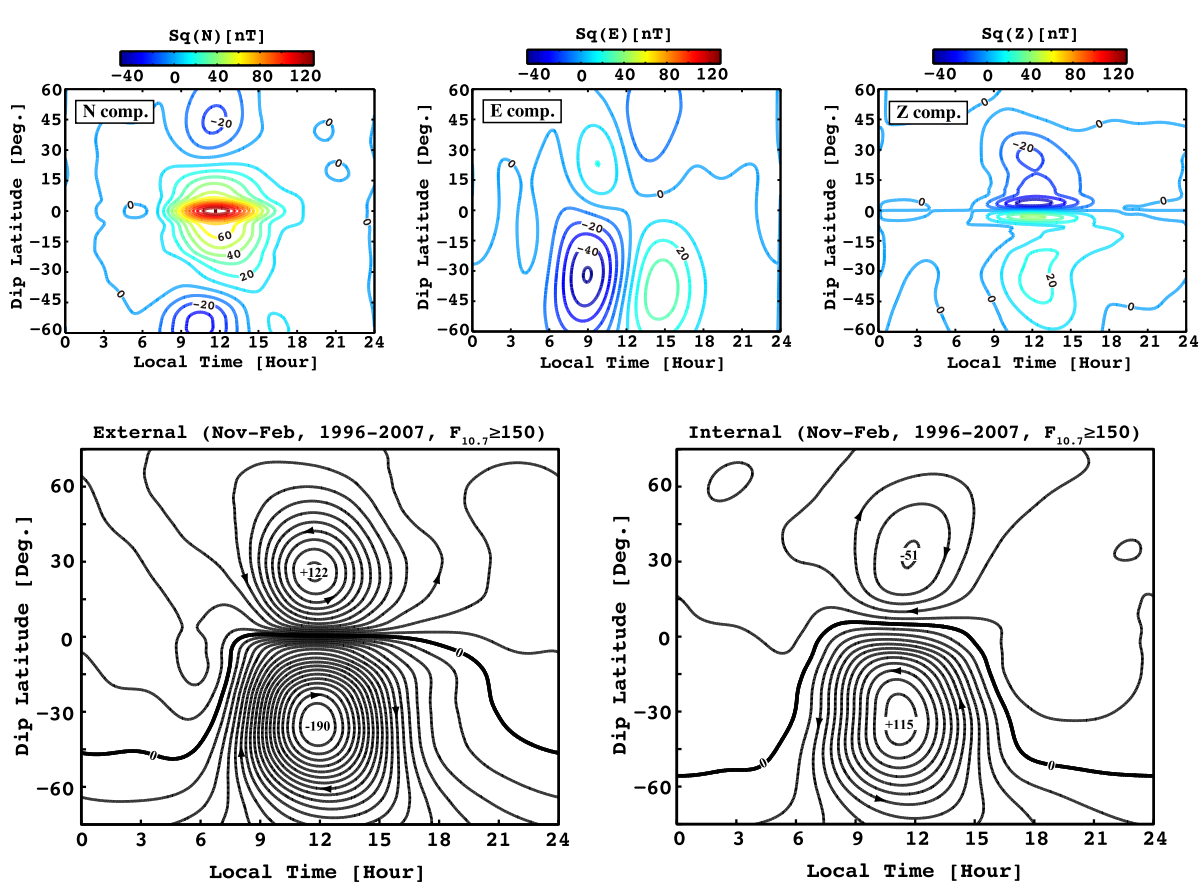

Fig. 23 (Top) Sq field in the magnetic-northward $(N)$, magnetic-eastward $(E)$, and vertical $(Z)$ component in the East Asian sector averaged over the northern winter months (November-February) of 1996-2007 under high solar activity conditions $\left(F_{10.7} \geq 150 \mathrm{sfu}\right.$ ). (Bottom) External and internal components of the equivalent Sq current system with an contour interval of $10 \mathrm{kA}$. From Yamazaki (2011)

the magnetic-northward $(N)$ and vertical $(Z)$ components. Since the Sq field is well constrained in the slice method, it is possible to include high-degree terms of the spherical functions. The truncation level is typically chosen to be around $n=10-20$, but needs to be extended to $n=40$, or so, in order to resolve the equatorial electrojet (e.g., Suzuki 1973; Yamazaki 2011). The magnetic potentials and current functions can be calculated using the same least-squares technique as the instantaneous method. Figure 23 (bottom) gives examples of external (left) and internal (right) current systems. It may be noticed that the internal current system has a very similar pattern as the external current system, but the direction of the current is the opposite and the strength of the current is approximately one half.

When Sq data are available only from a particular region of the world, the global analysis techniques described above do not work properly. Haines and Torta (1994) introduced a technique called spherical cap harmonic analysis, which enables the separation of the external and internal potentials based on a regional analysis of the Sq field. The spherical cap harmonic analysis is similar to the normal spherical harmonic analysis, but it does not analyze a global field. Instead, the analysis is made within a spherical cap that covers the target region only. Torta et al. (1997) used the technique to investigate the behavior of the Sq current system over Europe. The technique was also used by Stening (2008) for the Sq current system over Australia. An example is presented in Fig. 24 for the external current system over Australia derived using the spherical cap harmonic analysis (after Stening 2008).

There are also other techniques that enable the separation of the internal and external Sq fields. Price and Wilkins (1963) used the surface integral method, which was developed by 


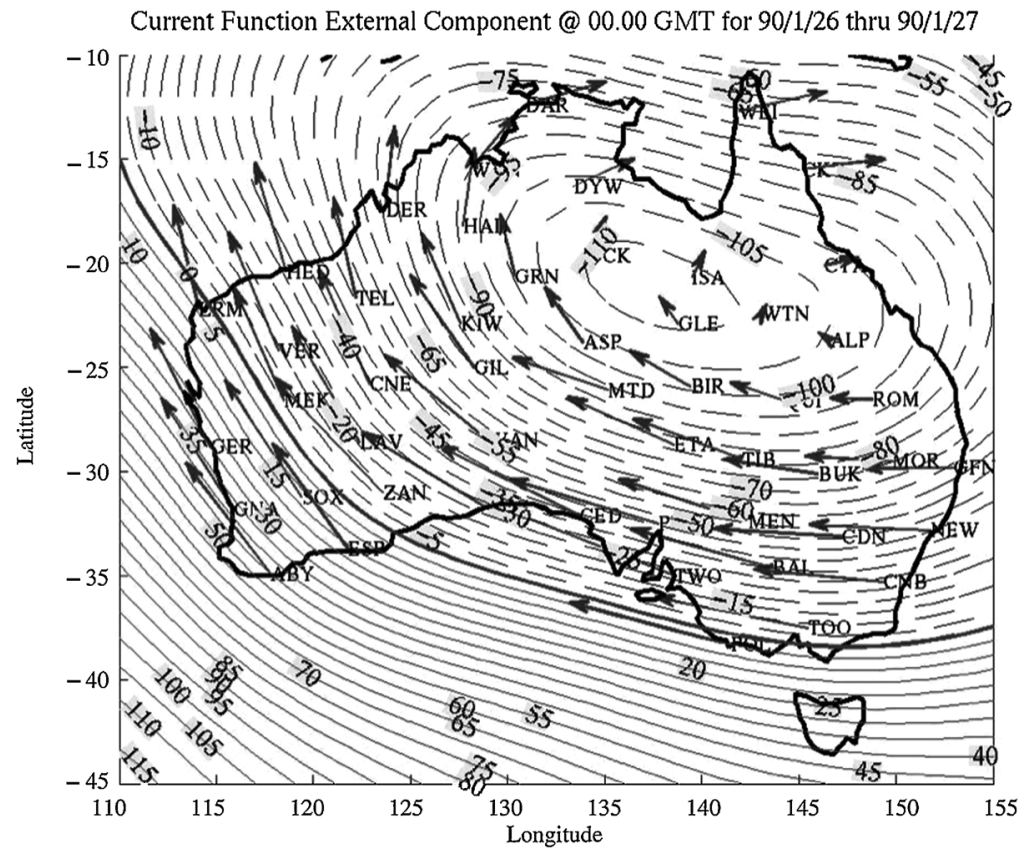

Fig. 24 An equivalent ionospheric Sq current system at 0800 UT on 27 January 1990. The arrows are derived using the method described in Sect. 3.6, while the contours are derived by spherical cap harmonic analysis. The contour intervals are $5 \mathrm{kA}$. From Stening (2008)

Vestine (1941). Koch and Kuvshinov (2013, 2015) have developed an inversion technique called S3D. The S3D uses a 3-D conductivity model of the Earth and ocean as an input. Chulliat et al. (2013) also introduced an inversion technique that takes into account the conductivity of the mantle and oceans. Their technique is suitable to include satellite data as well as ground measurements. Sabaka et al. (2002) used a "comprehensive approach", which simultaneously determines the magnetic potentials for magnetospheric, ionospheric, and underground sources by the use of satellite and ground data. The ionospheric source represents the Sq-EEJ current system. Figure 25 shows the ionospheric current system derived from the latest version of their model, CM5 (Sabaka et al. 2015). Note how the currents are constrained by the magnetic equator.

\subsection{Determination of Sq Current Intensity}

The current intensity is one of the quantities that characterize the Sq current system. One way to evaluate the $\mathrm{Sq}$ current intensity is to calculate the difference in the values of the current function at the northern and southern $\mathrm{Sq}$ foci; this quantity may be called total $S q$ current intensity $J_{\text {total }}$ (e.g., Yamazaki 2011) as it represents the total amount of equivalent $\mathrm{Sq}$ currents that flow in the region between the two Sq foci. However, as described in the previous section, the determination of the Sq current function by spherical harmonic analysis requires a global data set, which is not always available. An easy alternative is to analyze the $Y$-component (or magnetic-eastward $E$-component) $\mathrm{Sq}$ variation at a middle-latitude station which is located near the $\mathrm{Sq}$ focus. In the Northern Hemisphere, $\Delta Y$ is positive in the morning owing to southward currents and negative in the afternoon owing to northward 

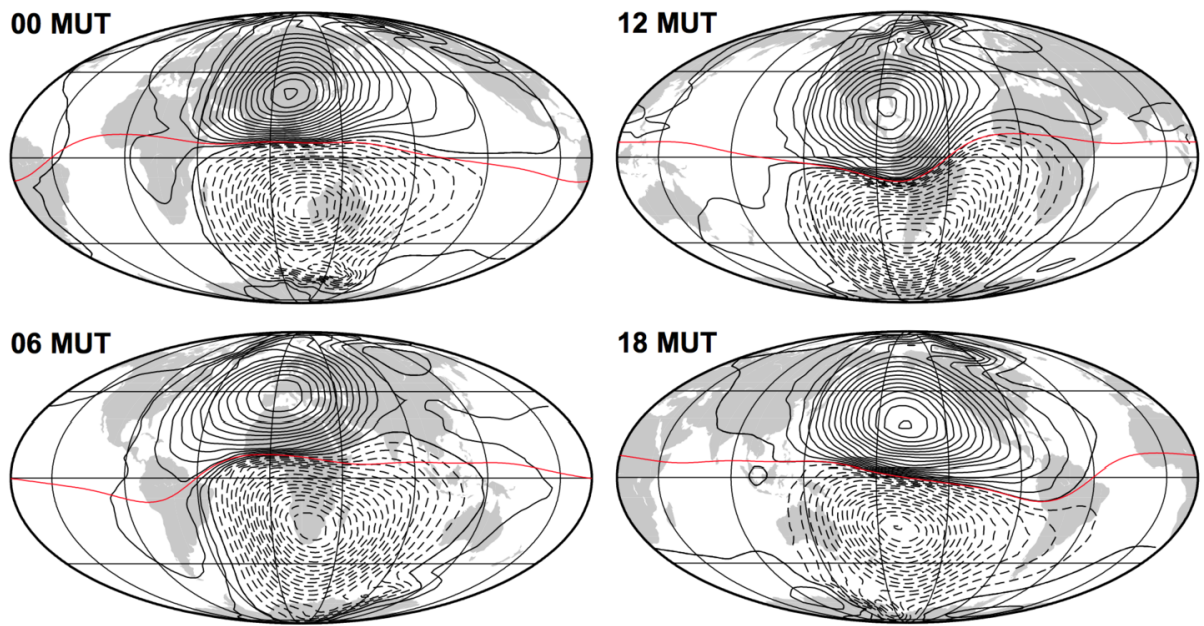

Fig. 25 Ionospheric current systems derived from CM5 for March equinox and moderate solar activity conditions at different times of day. The contour intervals are $10 \mathrm{kA}$. From Sabaka et al. (2015). MUT is the magnetic universal time $t_{m}=\left(180-\phi_{d, s}\right) / 15$, where $\phi_{d, s}$ is the dipole longitude of the sub-solar point

currents. (The pattern is the opposite in the Southern Hemisphere.) Using (38), the total current that flows around the northern Sq vortex may be estimated as follows:

$$
J_{N}=\frac{2 \pi\left(R_{E}+h\right) \cos (\lambda)}{24} \sum_{L T=6}^{18} \frac{2}{3} \frac{10}{2 \pi} \frac{|\Delta Y(L T)|}{2} 10^{-3},
$$

where $J_{N}$ is the Sq current intensity in the Northern Hemisphere in kA, $R_{E}$ is the Earth's radius in $\mathrm{km}, h$ is the height of the dynamo region in $\mathrm{km}, \lambda$ is the magnetic latitude of the station, and $L T$ is local time in hour. The summation of $\Delta Y$ is performed over the daytime period (0600-1800 LT). It is then divided by two, because the direction of the current changes from southward to northward around midday. The factor before the summation represents the horizontal length of the ionosphere corresponding to 1 hour. Takeda (2015) used a similar formula to calculate the Sq current intensity in the Northern Hemisphere. The Sq current intensity in the Southern Hemisphere $J_{S}$ can be similarly estimated, so that $J_{\text {total }}=J_{N}+J_{S}$.

When geomagnetic activity is high, ground-level magnetic perturbations are substantially influenced by magnetospheric currents, which makes it difficult to evaluate the intensity of Sq currents. Hibberd (1981) introduced a simple technique to deal with this problem. His technique involves a pair of stations that have the same longitude but different latitudes; one station is on the equatorial side of a Sq focus and the other station is on the polar side of the Sq focus. The difference in the horizontal magnetic field $H$ is calculated, and the daily range of the resulting variation in the difference is used as a proxy of the current intensity $J_{N}$ or $J_{S}$. During the daytime, $H$ at the station on the equatorial side of the Sq focus is subject to the effect of eastward Sq currents and the effect of magnetospheric currents. On the other hand, $H$ at the station on the polar side of the $\mathrm{Sq}$ focus undergoes the effect of westward Sq currents and the effect of magnetospheric currents. Since the effects of the magnetospheric currents are nearly identical at the two stations, the residual $H$ field is largely free from disturbances of magnetospheric origin (Xu 1992). Hibberd's technique was 
used by various authors to study the variability of the Sq current intensity (e.g., Briggs 1984; Takeda and Araki 1985; Hibberd 1985; Stening 1995b).

\subsection{Determination of Sq Focus Position}

The position of Sq current foci is another important quantity to characterize the Sq current system. If the external Sq magnetic potential $M_{e x}$ is determined from the spherical harmonic analysis (Sect. 3.7) or other techniques, the Sq current foci can be defined as the maximum and minimum points of $M_{e x}$. There are also other methods to estimate the position of Sq foci without using the magnetic potential (e.g., Gupta 1973; Tarpley 1973). Stening et al. (2005a) assessed the performance of various methods that had been proposed in earlier studies. The technique they found the most accurate takes the following two steps: (1) determine the time when $\Delta Y$ crosses the zero level and (2) plot $\Delta X$ at that time as a function of latitude and draw a regression line to find the latitude where $\Delta X$ crosses zero, which gives the latitude of the $\mathrm{Sq}$ focus. The technique requires a north-south chain of magnetometers (four stations or more) in order to work properly. Stening et al. (2005b, 2007) and Torta et al. (2010) used this technique to investigate the variability of the Sq focus position (see also Sect. 4 of the present paper).

\subsection{Determination of $L$}

In order to accurately describe the daily variation of the geomagnetic field, it is necessary to evaluate the lunar variation $\mathrm{L}$. The determination of $\mathrm{L}$ requires two quantities among $(t, \tau, \nu)$, where $t$ is solar time (or more commonly known as local time), $\tau$ is lunar time, and $v$ is lunar phase (also known as lunar age). Figure 26 (after Forbes et al. 2013) illustrates how $t, \tau$, and $v$ are defined at a point $P$ on the Earth. As can be seen in Fig. 26, the three parameters are related to each other by

$$
t=\tau+v .
$$

In units of hours, the lunar phase $v$ is equal to zero at new moon and is equal to 12 at full moon. One cycle of the lunar phase corresponds to 29.5306 solar days. A list of $\tau$ and $v$ values for the years 1850-2050 can be found in the paper by Sugiura and Fanselau (1966), along with the formula for calculating $\tau$ and $v$.

Fig. 26 Schematic illustrating the relations among solar (local) time $t$, lunar time $\tau$, and lunar age $v$ for a point $P$ on the Earth. From Forbes et al. (2013)

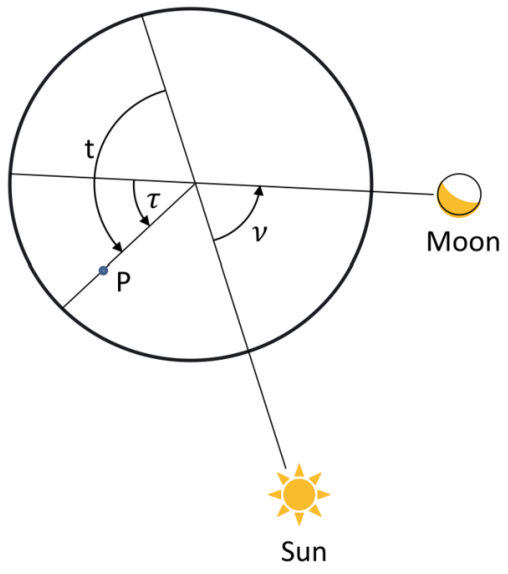



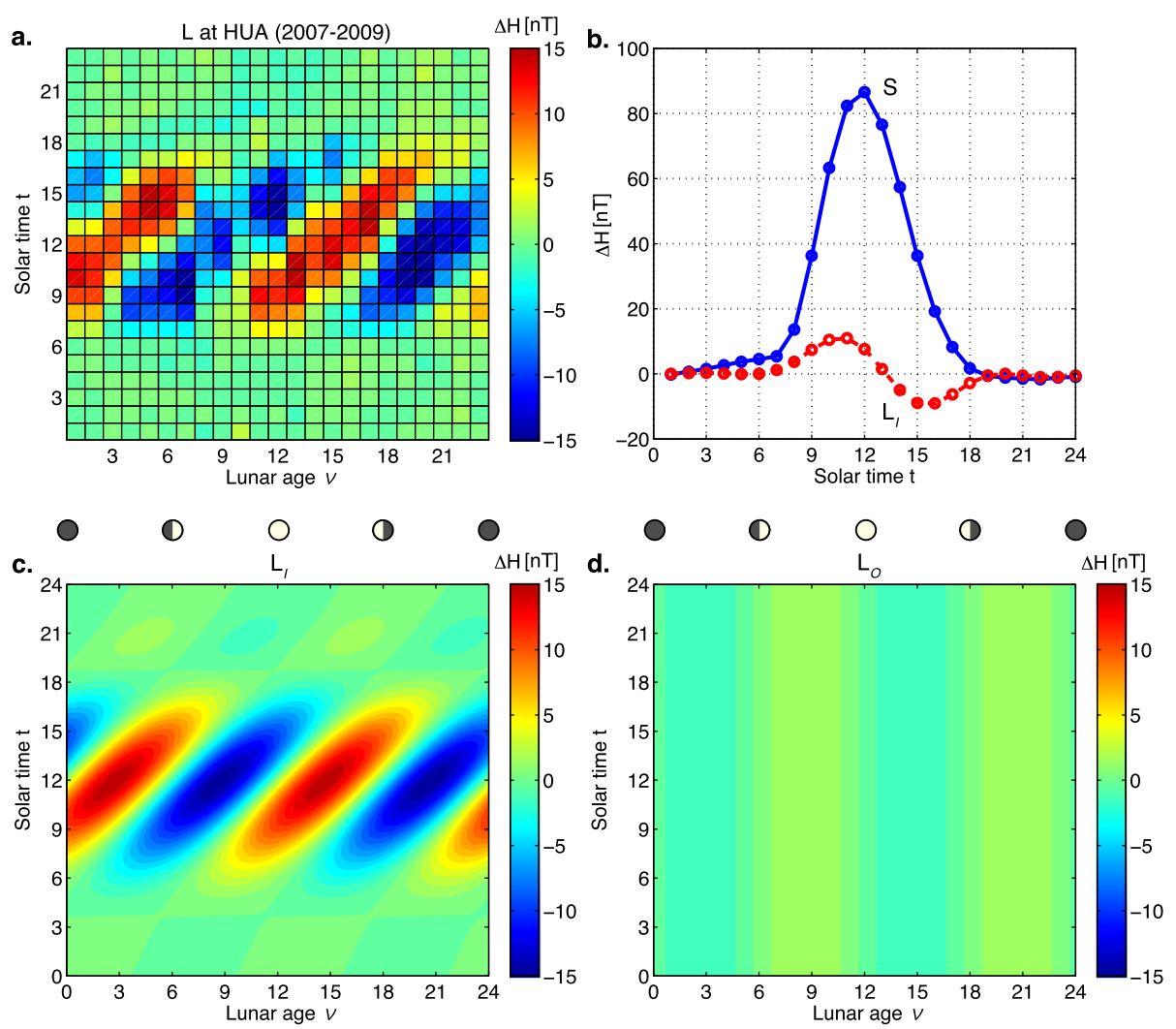

Fig. 27 Geomagnetic lunar variation at Huancayo $\left(12.0^{\circ} \mathrm{S}, 75.3^{\circ} \mathrm{W}\right)$ during 2007-2009. (a) The average lunar variation as a function of solar time and lunar age. (b) A comparison between the solar variation and luni-solar variation at new moon (exclude the oceanic component). (c) The luni-solar variation excluding the oceanic component. (d) The oceanic component of the luni-solar variation

In Fig. 27a, we present the average lunar variation in $H$ at Huancayo for the period 20072009. The results were obtained by the following procedures. First, the main field and Dst field were evaluated and removed from $H$ using the method described in Sect. 3.3. Next, the solar variation was evaluated at each local time for each day as the average value over \pm 14 days at the same local time, and removed form the data. Finally, the residuals are grouped into hourly $t$ and $v$ bins, and then averaged. The results in Fig. 27a reveal a prominent semimonthly variation. That is, at a fixed solar time $t$, there are two peaks and troughs within a cycle of the lunar phase. The amplitude and phase of the semi-monthly variation change with solar time. At a fixed lunar phase $v$, a semidiurnal change dominates the daily variation. As the lunar phase progresses, the phase of the semidiurnal lunar variation shifts to later local times. This is owing to the difference in the lengths of a solar day $(=24.00 \mathrm{~h})$ and a lunar day $(=24.84 \mathrm{~h})$. The phase of the daily variation of $\mathrm{L}$ tends to be fixed at a certain lunar time $\tau$, not solar time $t$ (e.g., Rastogi and Trivedi 1970; Yamazaki et al. 2011b). It is obvious from Fig. 27a that the lunar variation is strongly coupled to the solar daily variation imposed by the ionospheric conductivity change. For this reason, the geomagnetic lunar variation is also called luni-solar variation. 
Mathematical formula for the lunar variation have been developed in the past (e.g., Chapman and Bartels 1940, p. 245; Chapman and Miller 1940; Malin and Chapman 1970; Winch and Cunningham 1972). Most commonly, L variations are expressed by the following equation, which is known as Chapman's phase law (after Chapman 1919):

$$
L(t, \tau)=\sum_{n=1}^{4} l_{n} \sin \left\{(n-2) \frac{2 \pi}{24} t+2 \frac{2 \pi}{24} \tau+\lambda_{n}\right\}
$$

or using $t$ and $v$,

$$
L(t, v)=\sum_{n=1}^{4} l_{n} \sin \left(n \frac{2 \pi}{24} t-2 \frac{2 \pi}{24} v+\epsilon_{n}\right) .
$$

Thus at a fixed $t$, L changes semi-monthly with $v$. At a fixed $v, \mathrm{~L}$ changes with $t$. Equation (76) can also be written as

$$
L\left(t^{\prime}, v^{\prime}\right)=\sum_{n=1}^{4}\left\{a_{n} \cos \left(n t^{\prime}-2 v^{\prime}\right)+b_{n} \sin \left(n t^{\prime}-2 v^{\prime}\right)\right\}
$$

where $t^{\prime}$ and $v^{\prime}$ are hour angles (in radians) for solar time and lunar age, respectively. Using magnetic data expressed as a vector $\mathbf{d}_{\mathbf{L}}$,

$$
\mathbf{d}_{\mathbf{L}}=\left(\begin{array}{c}
d_{1} \\
d_{2} \\
d_{3} \\
\vdots \\
d_{k}
\end{array}\right)
$$

at solar time $t^{\prime}\left(=t_{1}^{\prime}, t_{2}^{\prime}, \ldots, t_{k}^{\prime}\right)$ with corresponding lunar age $v^{\prime}\left(=v_{1}^{\prime}, v_{2}^{\prime}, \ldots, v_{k}^{\prime}\right),(77)$ can be expressed by the matrix equation

$$
\mathbf{d}_{\mathbf{L}}=\mathbf{A}_{\mathbf{L}} \mathbf{M}_{\mathbf{L}}
$$

where $\mathbf{A}_{\mathbf{L}}$ is a matrix of Fourier functions

$$
\mathbf{A}_{\mathbf{L}}=\left(\begin{array}{ccccccc}
\cos \left(1 t_{1}^{\prime}-2 v_{1}^{\prime}\right) & \cos \left(2 t_{1}^{\prime}-2 v_{1}^{\prime}\right) & \ldots & \cos \left(4 t_{1}^{\prime}-2 v_{1}^{\prime}\right) & \sin \left(1 t_{1}^{\prime}-2 v_{1}^{\prime}\right) & \ldots & \sin \left(4 t_{1}^{\prime}-2 v_{1}^{\prime}\right) \\
\cos \left(1 t_{2}^{\prime}-2 v_{2}^{\prime}\right) & \cos \left(2 t_{2}^{\prime}-2 v_{2}^{\prime}\right) & \ldots & \cos \left(4 t_{2}^{\prime}-2 v_{2}^{\prime}\right) & \sin \left(1 t_{2}^{\prime}-2 v_{2}^{\prime}\right) & \ldots & \sin \left(4 t_{2}^{\prime}-2 v_{2}^{\prime}\right) \\
\cos \left(1 t_{3}^{\prime}-2 v_{3}^{\prime}\right) & \cos \left(2 t_{3}^{\prime}-2 v_{3}^{\prime}\right) & \ldots & \cos \left(4 t_{3}^{\prime}-2 v_{3}^{\prime}\right) & \sin \left(1 t_{3}^{\prime}-2 v_{3}^{\prime}\right) & \ldots & \sin \left(4 t_{3}^{\prime}-2 v_{3}^{\prime}\right) \\
\vdots & \vdots & \ddots & \vdots & \vdots & \ddots & \vdots \\
\cos \left(1 t_{k}^{\prime}-2 v_{k}^{\prime}\right) & \cos \left(2 t_{k}^{\prime}-2 v_{k}^{\prime}\right) & \ldots & \cos \left(4 t_{k}^{\prime}-2 v_{k}^{\prime}\right) & \sin \left(1 t_{k}^{\prime}-2 v_{k}^{\prime}\right) & \ldots & \sin \left(4 t_{k}^{\prime}-2 v_{k}^{\prime}\right)
\end{array}\right)
$$


and $\mathbf{M}_{\mathbf{L}}$ is a vector of the coefficients

$$
\mathbf{M}_{\mathbf{L}}=\left(\begin{array}{c}
a_{1} \\
a_{2} \\
\vdots \\
a_{4} \\
b_{1} \\
\vdots \\
b_{4}
\end{array}\right) .
$$

The least-squares solution for (79) can be found as

$$
\mathbf{M}_{\mathbf{L}}=\left\{\left(\mathbf{A}_{\mathbf{L}}\right)^{\mathrm{T}} \mathbf{A}_{\mathbf{L}}\right\}^{-1}\left\{\left(\mathbf{A}_{\mathbf{L}}\right)^{\mathrm{T}} \mathbf{d}_{\mathbf{L}}\right\}
$$

The mathematical expression of $\mathrm{L}$ can be derived by putting these coefficients into (77).

In addition to the ionospheric currents and the secondary currents induced in the Earth and sea, the effect of ocean dynamo also contributes to the $\mathrm{L}$ variations on the ground. The ocean dynamo arises due to tidal movements of the sea across the geomagnetic field. Malin (1970) postulated that the conductivity of the ocean is independent of solar time while the ionospheric conductivity changes significantly in the course of a day. Thus, the electric currents resulting from the ocean dynamo do not depend on solar time $t$ but depend on lunar time $\tau$. In (75), the component of $\mathrm{L}$ that is independent of $t$ can be readily found by substituting $t=0$. That is,

$$
L_{O}(t, \tau)=\sum_{n=1}^{4} l_{n} \sin \left(2 \frac{2 \pi}{24} \tau+\lambda_{n}\right) .
$$

The ionospheric contributions are thus,

$$
L_{I}=L-L_{O}
$$

Malin's technique has been used in many studies to evaluate the ocean dynamo effect (e.g., Sastri and Rao 1971; Shiraki 1978, 1981; Stening and Winch 1979; McKnight 1995; Çelik et al. 2012). Satellite magnetic measurements confirmed the presence of lunar tidal signals associated with the ocean dynamo during night (Tyler et al. 2003; Maus and Kuvshinov 2004; Sabaka et al. 2016).

Figures $27 \mathrm{c}$ and $27 \mathrm{~d}$ show $L_{I}$ and $L_{O}$, respectively, derived using the technique described above. The ionospheric effect $L_{I}$ is much larger than the oceanic effect $L_{O}$. The $L_{I}$ component well reproduces the average lunar variation shown in Fig. 27a. Figure 27b compares the average solar and lunar variations at new moon. The amplitude ratio of $L$ to $S$ is approximately 0.15 in this particular case. It may be inferred from the figure that the lunar variation can be a cause of the counter electrojet (Sect. 2.4), when the lunar variation is large or when the solar variation is weak, or both. It is noted, however, that the separation between the solar and lunar variations is not possible on the day-to-day basis. The determination of L requires a full coverage of solar and lunar times, which takes at least one lunar cycle $(=29.5306$ days). Thus, the contribution of $\mathrm{L}$ to a particular counter electrojet event cannot be determined. The analysis of $\mathrm{L}$ is usually made with the data covering several months or longer. When the global L field is determined, it is possible to apply the spherical harmonic analysis so as to separate the external and internal sources (e.g., Matsushita and Maeda 1965b; 
Malin 1973; Winch 1981; Matsushita and Xu 1984; Yamazaki 2014; Çelik 2014). Figure 5 shows the external L current systems presented by Çelik (2014). The ratio of the external to internal magnetic potentials is similar between Sq and $\mathrm{L}$.

\subsection{EEJ Intensity and Equatorial F-Region Parameters}

The electric fields caused by the E-region wind dynamo are transmitted to the F-region along equipotential magnetic field lines. At F-region heights $(150-500 \mathrm{~km})$, where the plasmaneutral collision frequency is very small, the velocity of the plasma flow can be approximated by $(\mathbf{E} \times \mathbf{B}) /|\mathbf{B}|^{2}$ or the so-called $\mathbf{E} \times \mathbf{B}$ drift. Here, $\mathbf{E}$ is the electric field and $\mathbf{B}$ is the geomagnetic field. In the dayside equatorial ionosphere, where $\mathbf{B}$ is horizontal and $\mathbf{E}$ is eastward, the $\mathbf{E} \times \mathbf{B}$ drift is upward and thus plasma particles are vertically transported upward across the geomagnetic field. The lifted plasma eventually diffuses down along magnetic field lines. These plasma transport processes are often referred to as the equatorial plasma fountain. A result of the equatorial plasma fountain is the formation of a "trough" of reduced F-region plasma density over the magnetic equator and two "crests" of enhanced plasma density at about $\pm 18^{\circ}$ away from the magnetic equator. This characteristic distribution of the F-region plasma is known as the equatorial ionization anomaly (EIA).

Radar and geomagnetic field measurements have made significant contributions to the understanding of equatorial electrodynamic coupling between the E-region and F-region ionosphere. Balsley and Woodman (1969), using simultaneous measurements of the Eregion zonal electric field and the F-region vertical plasma drift over the Jicamarca radar $\left(12.0^{\circ} \mathrm{S}, 76.9^{\circ} \mathrm{W}\right)$, found a remarkable correlation between the two quantities. Nair et al. (1970) and Chandra et al. (1971) showed a linear correlation between the midday values of the E-region zonal electric field over the Thumba $\operatorname{radar}\left(8.5^{\circ} \mathrm{N}, 76.9^{\circ} \mathrm{E}\right)$ and the daily range in $H$ near the magnetic equator. The correlation was shown to be better when the difference in $H$ at Trivandrum $\left(8.5^{\circ} \mathrm{N}, 77.0^{\circ} \mathrm{E}\right)$ and Alibag $\left(18.6^{\circ} \mathrm{N}, 72.9^{\circ} \mathrm{E}\right)$ was used than when $H$ only at Trivandrum was used. Rastogi and Patil (1986) showed that the difference in $H$ between Trivandrum minus Alibag correlates well with the E-region zonal electric field not only during quiet periods but also during geomagnetically disturbed times. As discussed in Sect. 2.5, taking the difference in $H$ between equatorial and off-equatorial stations removes a large portion of magnetospheric contributions from the data, and thus effectively isolates the ionospheric contributions. As described also in Sect. 2.5, there is a debate concerning whether Sq and EEJ are separate current systems. Some authors consider that taking the difference in $H$ between equatorial and off-equatorial stations separates the two contributions, and the residual represents a pure contribution of EEJ.

Building upon those early studies, Anderson et al. (2002) proposed empirical formulae to describe the relationship between the equatorial F-region vertical plasma drift velocity measured by the Jicamarca radar and the difference in $H$ measured by a pair of magnetometers; equatorial station Canete $\left(13.1^{\circ} \mathrm{S}, 76.4^{\circ} \mathrm{W}\right)$ and off-equatorial station Piura $\left(5.2^{\circ} \mathrm{S}\right.$, $80.6^{\circ} \mathrm{W}$ ). Anderson et al. (2004) made further efforts to improve these empirical formulae. One of the formulae presented by Anderson et al. (2004) is,

$$
\begin{aligned}
V_{z}= & -1989.51+1.002 \text { year }-0.00022 \mathrm{DoY}-0.0222 \mathrm{FD} \\
& -0.0282 \mathrm{FA}-0.0229 \mathrm{Ap}+0.0589 \mathrm{Kp} \\
& -0.3661 \mathrm{LT}+0.1865 \Delta \mathrm{H}+0.00028 \Delta \mathrm{H}^{2}-0.0000023 \Delta \mathrm{H}^{3},
\end{aligned}
$$

where $V_{z}$ is the upward $\mathbf{E} \times \mathbf{B}$ drift velocity in $\mathrm{m} / \mathrm{s}$; DoY is the day of year; FD is the daily solar activity index $F_{10.7}$; FA is the 81-day average value of FD; Ap and $\mathrm{Kp}$ are daily and 
3-hourly geomagnetic indices; LT is the local time; and $\Delta \mathrm{H}$ is the difference in $H$ at Jicamarca $\left(11.9^{\circ} \mathrm{S}, 76.9^{\circ} \mathrm{W}\right)$ and Piura, measured from the nigthtime baseline. Fang et al. (2008) numerically validated the linear relationship between the F-region vertical plasma drift velocity and the difference in the $H$-component magnetic perturbations at equatorial and off-equatorial stations for different levels of solar activity. Anderson et al. (2006) suggested that the empirical formulae derived from the Peruvian observations can be applied to the magnetic data from the Philippine longitude sector. Similarly, Anghel et al. (2007) applied the technique to the data from the Indian longitude sector, and Yizengaw et al. (2011, 2012) to the data from the African longitude sector. It is noted that the $\mathbf{E} \times \mathbf{B}$ drift velocity estimated at longitudes other than the Peruvian sector should be interpreted with caution because the validation of the technique has been made only for limited cases. For selecting the pair of stations for the estimation of the equatorial F-region vertical plasma drift velocity, Anderson et al. (2004) suggested that the equatorial station should be located within $\pm 2^{\circ}$ from the magnetic equator and the off-equatorial station should be located $6^{\circ}-9^{\circ}$ away from the magnetic equator.

Evidence for the electrodynamic coupling between the E-region and F-region ionosphere has also been found in plasma density data in the equatorial anomaly region. Studies have shown that the intensity and latitude of the equatorial anomaly crests vary with the intensity of the equatorial electrojet (Dunford 1967; MacDougall 1969; Rastogi and Rajaram 1971; Rush and Richmond 1973; Raghavarao et al. 1978; Balan and Iyer 1983; Huang et al. 1989; Rastogi and Klobuchar 1990; Rama Rao et al. 2006; Chen et al. 2008; Stolle et al. 2008). The most comprehensive study was made by Stolle et al. (2008), involving 5 years of CHAMP electron density observations along with radar and geomagnetic field measurements. The following are empirical formulae, given by Stolle et al. (2008), relating geomagnetic field perturbations to equatorial anomaly parameters:

$$
\begin{aligned}
& \mathrm{CTR}=1.39+9.7 \times 10^{-3} \Delta \mathrm{H}, \\
& \mathrm{L}_{\mathrm{c}}=1.092+0.5 \times 10^{-3} \Delta \mathrm{H},
\end{aligned}
$$

where CTR is the crest-to-trough ratio of the F-region electron density, i.e., a measure of the strength of the EIA; $\mathrm{L}_{\mathrm{c}}$ is the L-value of the flux tube containing the electron density crest, i.e., a measure of the latitudinal extent of the EIA; and $\Delta \mathrm{H}$ is the difference in $H$ at Huancayo $\left(12.0^{\circ} \mathrm{S}, 75.3^{\circ} \mathrm{W}\right)$ and Piura, with respect to the nighttime baseline, i.e., a measure of the equatorial electrojet intensity. These formulae were derived for CTR and $\mathrm{L}_{\mathrm{c}}$ measured $2 \mathrm{hrs}$ after $\Delta \mathrm{H}$ readings made between 0800 and 1500 hours local time. It is obvious from (86) and (87) that a stronger equatorial electrojet leads to a more prominent equatorial anomaly structure with its crests at higher latitudes. This is well illustrated in Fig. 28 (from Rama Rao et al. 2006), which shows maps of the total electron content for a strong, moderate, and weak electrojet days.

As mentioned in Sect. 2.1, the eastward daytime equatorial electric field sometimes shows a significant increase before it reverses to the nighttime westward direction. The strong eastward equatorial electric field during the pre-reversal enhancement is linked to another phenomena called equatorial spread-F or plasma bubble, which is characterized by a depletion of the equatorial ionospheric plasma density (e.g., Burke et al. 2004; Stolle et al. 2006). Attempts have been made by several authors (e.g., Sreeja et al. 2009; Uemoto et al. 2010; Hajra et al. 2012) to find a link between geomagnetic daily variations and the occurrence of the equatorial spread-F. For example, Uemoto et al. (2010) reported that the occurrence of the equatorial spread-F is reduced when the equatorial electrojet 1-2 hours prior to the sunset is westward. Their results suggest the possibility of the detection 

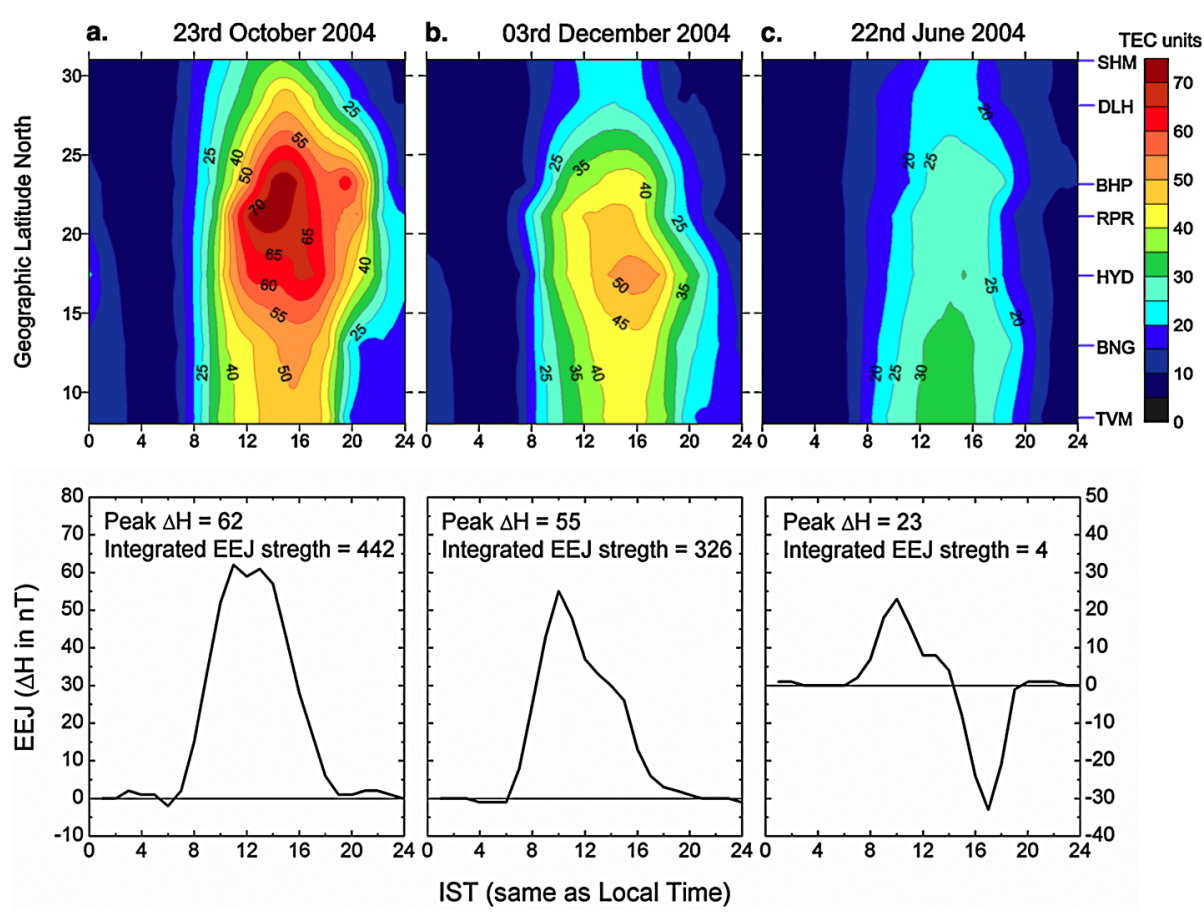

Fig. 28 Contour plots of the total electron content in the Northern Hemisphere at the Indian sector for (a) 23 October 2004, (b) 3 December 2004, and (c) 22 June 2004, along with the corresponding plots of the daily variation of the difference in $H$ between Tirunelveli and Alibag. From Rama Rao et al. (2006)

of the equatorial spread-F using ground magnetometer data. They analyzed the data in the Thailand sector during November 2007-October 2008. Similar investigations will be necessary for other regions and for other periods in order to establish the empirical relations between the equatorial electrojet and equatorial spread-F.

\section{Variability of Sq and EEJ}

The amplitude and phase of geomagnetic daily variations change over time, as the intensity and shape of the overhead current system change. In this section, we describe the variability of Sq and EEJ on various time scales, and address driving mechanisms. The variability of $\mathrm{L}$ is also addressed when it is important for the interpretation of Sq and EEJ.

\subsection{Solar Cycle Variation}

\subsubsection{Sq Current Intensity}

It is known for long time that the Sq amplitude varies with the sunspot number (e.g., Ellis 1898; Chapman and Bartels 1940, p. 220; Olsen 1993). The sunspot number is a proxy of solar extreme ultraviolet (EUV) radiation (wavelengths 10-120 nm), which causes the ionization of the Earth's upper atmosphere and produces the ionospheric plasma. Figure 29 (after Takeda 2013a) shows the daily range of the Sq variation in the $Y$ and $Z$ components 

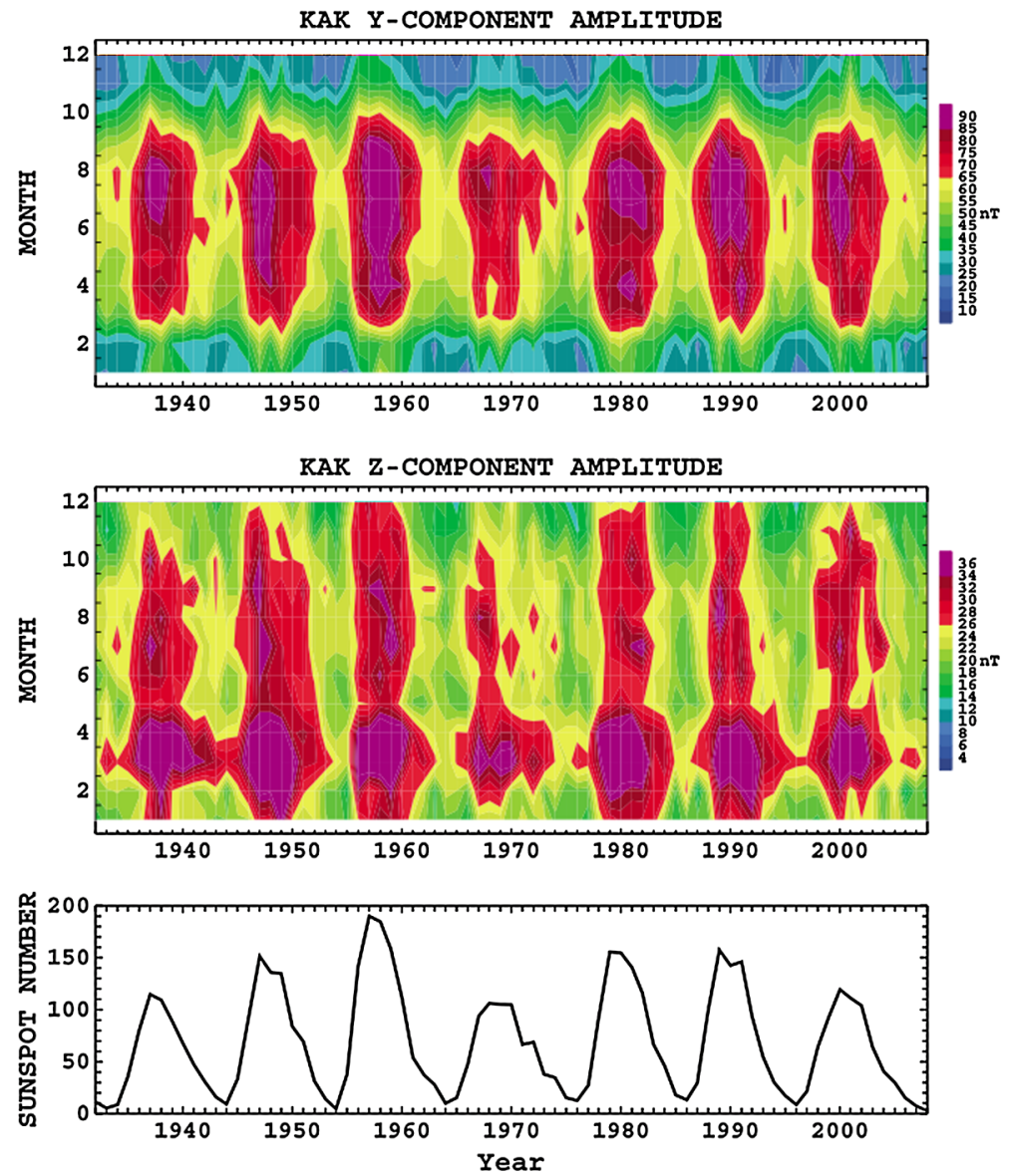

Fig. 29 The monthly average of the daily $\mathrm{Sq}$ range in the $Y$ and $Z$ components at Kakioka $\left(36.2^{\circ} \mathrm{N}\right.$, $140.2^{\circ} \mathrm{E}$ ), Japan from 1932 to 2008 , along with the annual sunspot number. From Takeda (2013a)

at Kakioka $\left(36.2^{\circ} \mathrm{N}, 140.2^{\circ} \mathrm{E}\right)$, Japan, along with the yearly average of the sunspot number. The solar control of $\mathrm{Sq}$ is evident in both components at all seasons. The total Sq current intensity $J_{\text {total }}$ (Sect. 3.8) during solar maximum is typically twice as large as that of solar minimum (Takeda 1999, 2002a). As discussed in Sect. 2.1, the solar cycle variation of Sq is primarily due to the change in the ionospheric conductivity.

Figure 30 reveals the dependence of the yearly-average Sq amplitude in $Y$ at Kakioka $\left(r Y_{\mathrm{KAK}}\right)$ on various solar indices during 1996-2015. For the calculation of the Sq amplitude, the $Y$-component $\mathrm{Sq}$ variation was first determined for each month. Then, the range (i.e., the difference between the maximum and minimum) of those Sq variations was used to compute the average value for each year. The top-left panel compares the amplitude of the Sq variation with the sunspot number. The Sq amplitude increases almost linearly with the sunspot number, leading to the high correlation $R=0.97$. Interestingly, a comparison of $r Y_{\mathrm{KAK}}$ with actual EUV measurements $(0.1-50 \mathrm{~nm})$ by the Solar EUV Monitor (SEM) spectrometer (Judge et al., 1998) on the Solar Heliospheric Observatory (SOHO) shows a poorer correlation $(R=0.91)$. Other solar proxies, namely, ground-based $F_{10.7}$ and satellite-based $M g I I$ indices also well correlate with $r Y_{\mathrm{KAK}} ; R=0.96$ and $R=0.97$, respectively. Table 4 

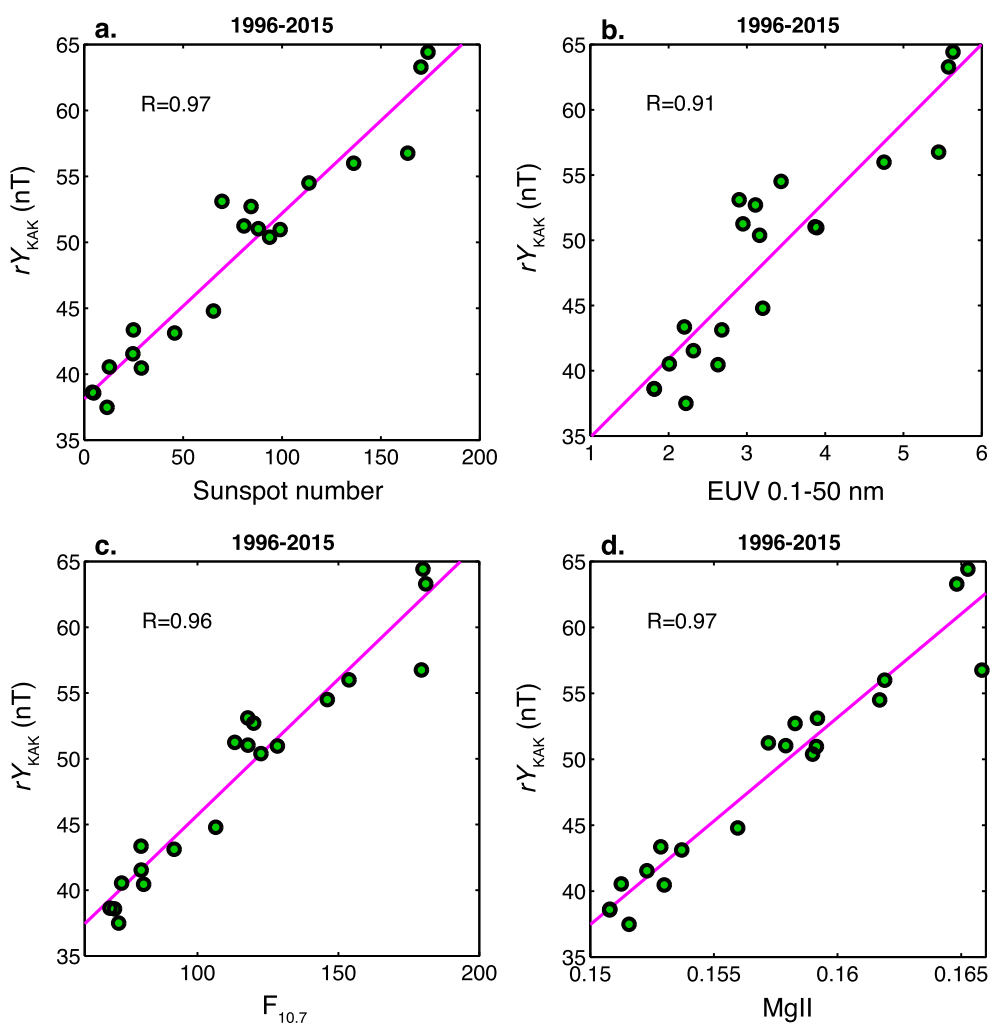

Fig. 30 The dependence of the range in the $Y$-component $\mathrm{Sq}$ variation at Kakioka $\left(36.2^{\circ} \mathrm{N}, 140.2^{\circ} \mathrm{E}\right)$, Japan on solar activity indices: (a) sunspot number, (b) EUV flux $(0.1-50 \mathrm{~nm}),(\mathbf{c}) F_{10.7}$ index, and (d) $M g I I$ index. Plotted are the yearly average values for 1996-2015

summarize the results for Kakioka, along with three other middle-latitude stations, namely, Honolulu (HON, 21.3 ${ }^{\circ} \mathrm{N}, 202.0^{\circ} \mathrm{E}$ ), Hawaii, San Juan (SJG, 18.1 ${ }^{\circ}, 293.9^{\circ} \mathrm{E}$ ), Puerto Rico, and Hermanus (HER, $34.4^{\circ} \mathrm{S}, 19.2^{\circ} \mathrm{E}$ ), South Africa. It can be seen in the table that the correlation is better when the Sq amplitude is averaged over longitude than when the Sq amplitude of individual stations is analyzed. This may be expected as the zonal average reduces the misfit caused by the limited longitudinal coverage of individual stations. Table 4 also shows that $\sqrt{\mathrm{EUV}}$ and $\sqrt{F_{10.7}}$ tend to give a better correlation to the Sq amplitude than EUV and $F_{10.7}$, respectively. Yamazaki and Kosch (2014) showed similar results using a different data set. They discussed how the ionospheric conductivity is proportional to the square root of the solar EUV flux, not to the EUV flux itself. Good agreement between the Sq amplitude and solar activity parameters presented in Table 4 suggests that those solar indices can be estimated or calibrated using Sq data (Svalgaard and Cliver 2007, Svalgaard 2016). However, such an application requires some caution; for example, as seen in Table 4, the average over longitude seems to be important for accurately estimating solar activity from $r Y$. Also, the sensitivity of the Sq amplitude to solar activity is different at different seasons, which makes it difficult to estimate solar activity from $r Y$ on a monthly basis (Mursula et al. 2009). Takeda (2002b) argued that the sensitivity of $r Y$ to solar activity is seasonal dependent because the relative contribution of inter-hemispheric field-aligned currents to $r Y$ varies with season. 
Table 4 Correlation coefficient $R$

\begin{tabular}{lllllll}
\hline & S.N. & EUV & $\sqrt{\mathrm{EUV}}$ & $F_{10.7}$ & $\sqrt{F_{10.7}}$ & $M g I I$ \\
\hline$r Y_{\mathrm{KAK}}$ & 0.97 & 0.91 & 0.92 & 0.96 & 0.97 & 0.97 \\
$r Y_{\mathrm{HON}}$ & 0.96 & 0.90 & 0.91 & 0.96 & 0.97 & 0.96 \\
$r Y_{\mathrm{SJG}}$ & 0.98 & 0.93 & 0.94 & 0.98 & 0.98 & 0.98 \\
$r Y_{\mathrm{HER}}$ & 0.97 & 0.93 & 0.93 & 0.97 & 0.97 & 0.97 \\
$r Y_{\text {mean }}$ & 0.99 & 0.94 & 0.95 & 0.99 & 0.99 & 0.99 \\
\hline
\end{tabular}

S.N. represents the annual sunspot number

$r Y_{\text {mean }}=\left(r Y_{\mathrm{KAK}}+r Y_{\mathrm{HON}}+r Y_{\mathrm{SJR}}+r Y_{\mathrm{HER}}\right) / 4$
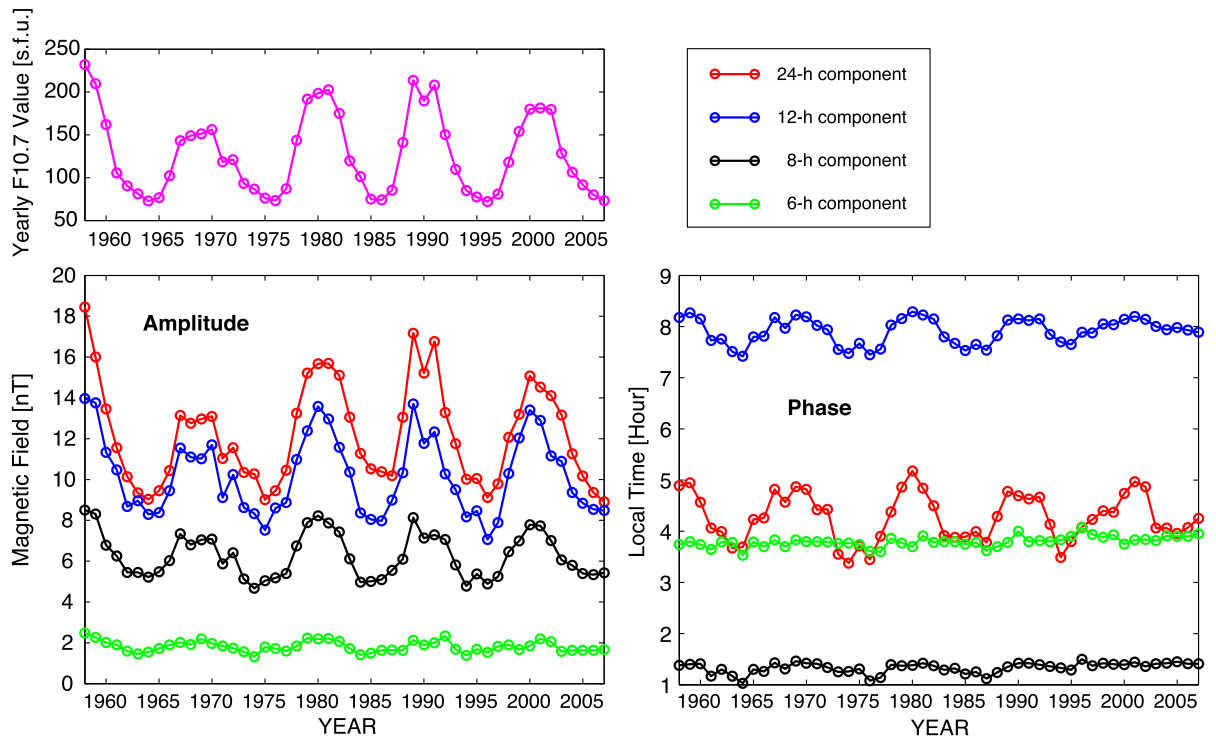

Fig. 31 Year-to-year variations in the amplitude and phase of the 24-, 12-, 8- and 6-hr Fourier components in $Y$ at Kakioka $\left(36.2^{\circ} \mathrm{N}, 140.2^{\circ} \mathrm{E}\right)$, Japan for $1958-2007$. The corresponding variation in the $F_{10.7}$ index is also indicated

\subsubsection{Sq Focus Positions}

Solar activity affects not only the intensity of Sq currents but also the position of Sq foci. Figure 31 displays the results of the Fourier analysis (Sect. 3.4) for the $Y$-component $\mathrm{Sq}$ variation at Kakioka. The amplitude of the 24-, 12-, and 8-hr Fourier components is strongly dependent on $F_{10.7}$. The solar cycle variation is also visible in the phase of the 24- and 12-hr components. That is, the phase of these Fourier components shifts to later local times as solar activity increases. Yamazaki and Kosch (2014) also found a similar solar cycle effect on the phase of Sq variations. Olsen (1993) showed that the Sq current foci move to later local times during solar maximum, which is consistent with our results in Fig. 31.

The solar activity effect on the latitude of Sq focus has been much less studied. Shiraki (1973) reported that the northern Sq current focus moves poleward during the solar minimum in the East Asian and North American regions. This feature was found in the winter 
Fig. 32 (a) The monthly average of the $F_{10.7}$ index during 1958-2007. (b) The northern Sq focus latitude in the East Asian sector; (blue) monthly values and (red) yearly running mean values (Color figure online)
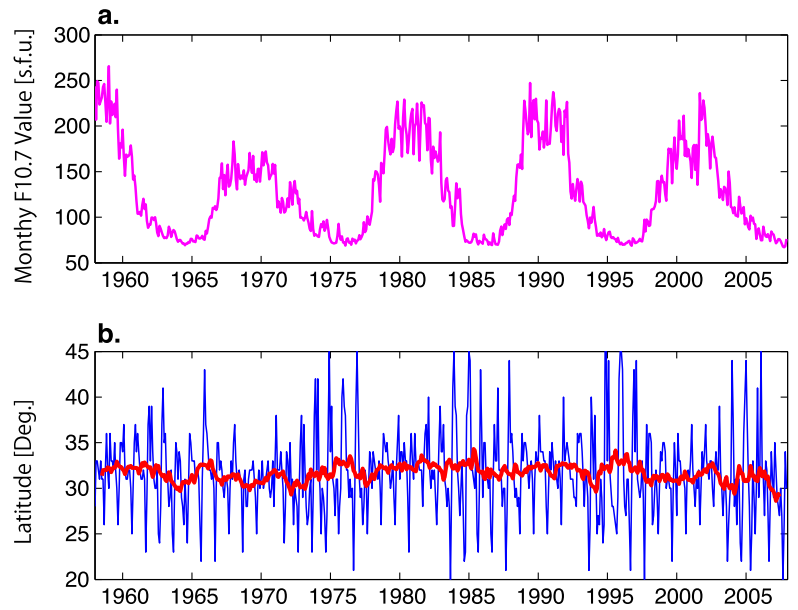

data (November-February), but not in the data for equinoxes (March, April, September, October) or summer (May-August). In other words, there is a solar activity dependence in the seasonal variation of the $\mathrm{Sq}$ focus latitude. We show in Fig. 32 the Sq focus latitude in the Northern Hemisphere during 1958-2007. The technique suggested by Stening et al. (2005a) (Sect. 3.9) was applied to the Sq variations observed in the Japanese longitude sector. The stations used are the following: Guam $\left(13.6^{\circ} \mathrm{N}, 144.9^{\circ} \mathrm{E}\right)$, Kanoya $\left(31.4^{\circ} \mathrm{N}, 130.9^{\circ} \mathrm{E}\right)$, Kakioka $\left(36.2^{\circ} \mathrm{N}, 140.2^{\circ} \mathrm{E}\right)$, and Memambetsu (43.9 $\left.{ }^{\circ} \mathrm{N}, 144.2^{\circ} \mathrm{E}\right)$. The Sq focus latitude was determined for each month, which is shown by the blue line in the bottom panel of Fig. 32. The red line in the same panel represents the 12-month average, which is fairly stable at $\sim 32^{\circ} \mathrm{N}$ during both solar maximum and solar minimum. It can be seen in Fig. 32 that the Sq focus latitude tends to show a larger seasonal variation during solar minimum than solar maximum. Fejer and Scherliess (2001) found that variability of the equatorial electric field is greater during solar minimum than solar maximum. Liu and Richmond (2013) discussed how the ionospheric variability due to atmospheric waves become more significant under solar minimum conditions than solar maximum conditions. Our results in Fig. 32 confirm Shiraki's finding that the seasonal variation of the Sq focus latitude depends on solar activity. Torta et al. (2010), also using the technique of Stening et al. (2005a), examined the Sq focus latitude in the South American longitude sector during 1964-1976 and 1997-2007. However, they did not find evidence for the solar cycle effect in the seasonal variation of the $\mathrm{Sq}$ focus latitude. Therefore, the solar activity dependence of the Sq focus latitude could be different in different regions.

\subsubsection{EEJ}

The intensity of the equatorial electrojet also shows a solar cycle variation (e.g., Rastogi et al. 1994; Alken and Maus 2007; Bhattacharyya and Okpala 2015). Yamazaki et al. (2010) demonstrated that the sensitivity of the equatorial electrojet to $F_{10.7}$ is approximately the same with the sensitivity of the global Sq current intensity to $F_{10.7}$. The time for the peak $\mathrm{EEJ}$ is around local noon during solar maximum and shifts to earlier local times during solar minimum (Rastogi and Iyer 1976). As mentioned in Sect. 4.1.2, the Sq current system also moves to earlier local times during solar minimum. Another important feature about the solar activity dependence of the equatorial electrojet is that the occurrence of the counter electrojet 
(Sect. 2.4) becomes more frequent during solar minimum in comparison to solar maximum (Rastogi 1974; Marriott et al. 1979; Chen et al. 1995). This is probably owing to the fact that the ionospheric dynamo is more subject to the atmospheric waves from the lower atmosphere during solar minimum (Liu and Richmond 2013). A counter electrojet event can be caused by solar and lunar tides of lower atmospheric origin. Fang et al. (2014) numerically studied a counter electrojet event on January 2009. They performed two simulations with the same tidal forcing but with different solar activity conditions; one for solar maximum and the other for solar minimum. It was shown that the reversal of the zonal electric field is more pronounced for the solar minimum case.

\subsubsection{L}

Studies have shown that the sensitivity of L currents to solar activity is different from that of Sq currents when the solar cycle influence is evaluated for the same spherical harmonic coefficients (Chapman et al. 1971; Malin et al. 1975; Olsen 1993; Çelik 2014). On that

Fig. 33 The 5-year mean amplitude of the (a) lunar semidiurnal variation and (b) solar semidiurnal variation for 1905-2010. The amplitude values are normalized to 100 . The green lines are results at individual stations, and the red lines are the average over the stations. The 5-year running average of the $F_{10.7}$ index is also presented in (c). From Yamazaki and Kosch (2014) (Color figure online) a.

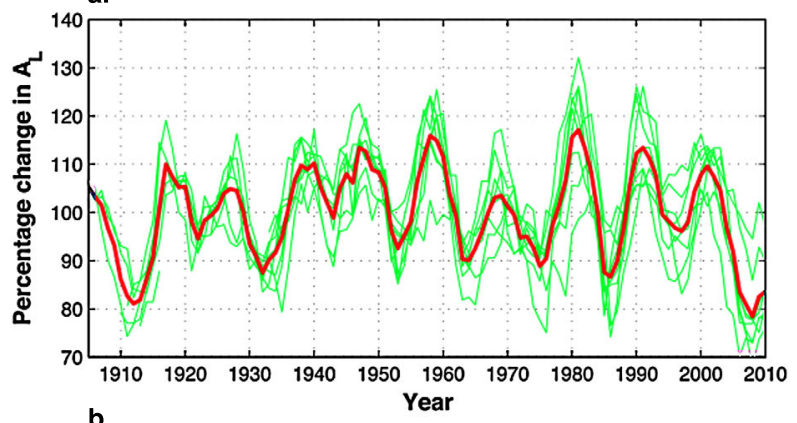

b.

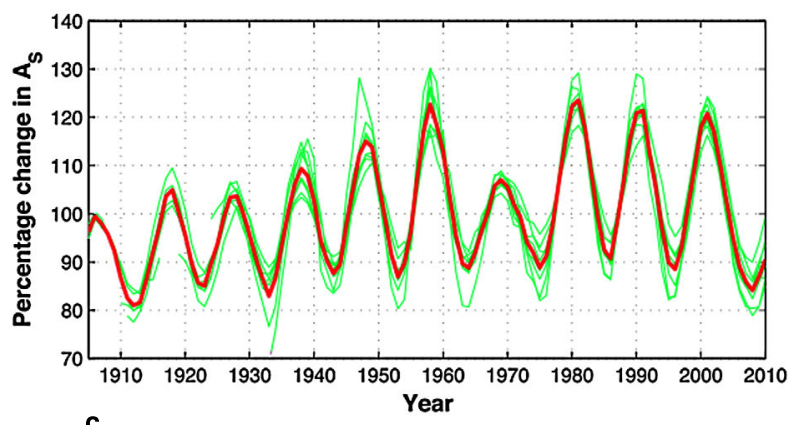

c.

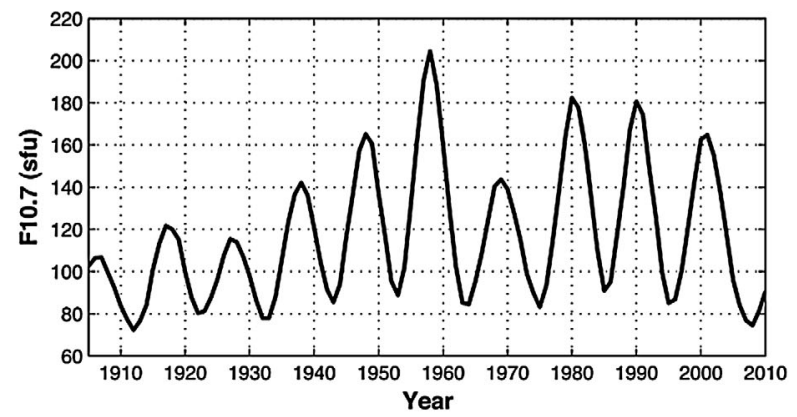


basis, Chapman et al. (1971) and Malin et al. (1975) suggested that L currents do not flow in the dynamo region of the ionosphere $(90-150 \mathrm{~km})$ as Sq currents but further aloft in the F-region ionosphere ( $300 \mathrm{~km})$. Butcher (1980) and Matsushita and Xu (1984) discussed that $\mathrm{L}$ currents flowing in the F region is improbable, and both $\mathrm{L}$ and $\mathrm{Sq}$ currents are likely to flow in the dynamo region. Yamazaki and Kosch (2014) evaluated the lunar semidiurnal variation using a slightly different method than previous authors. Instead of examining the luni-solar variation based on Chapman's phase law (Eq. (75)), they determined the lunar semidiurnal variation by simply ignoring the solar time. Figure 33 (after Yamazaki and Kosch 2014) shows their results. It can be seen that the solar cycle influence on the lunar and solar semidiurnal variations is similar.

\subsection{Seasonal Variation}

\subsubsection{Sq}

The pattern of the Sq variation slowly changes through the months of the year. The seasonal variation of $\mathrm{Sq}$ is dominated by annual (365.24-day) and semiannual (182.62-day) components. The annual component is often related to the Sq amplitude being greater during summer than winter at middle and high latitudes, while the semiannual component generally relates to the enhanced Sq amplitude during equinoctial months than other times at low and equatorial latitudes. Besides, the Sq variation at some locations shows a persistent asymmetry between the two equinoxes (Wulf 1963; Chulliat et al. 2005; Pham Thi Thu et al. 2011). Figure 34 (from Campbell 1982) illustrates the latitudinal distribution of the seasonal Sq change in the 24-, 12-, 8-, and 6-h Fourier components in the Northern Hemisphere. The semiannual variation is dominant at the magnetic equator in the magnetic-northward $N$ component, which is associated with the strong equatorial electrojet during equinoxes. The annual variation, which dominates the magneticeastward $E$ component, tends to be larger at higher latitudes, as the summer-winter difference in the ionization rate becomes more significant at higher latitudes. The annual variation in the Sq current intensity is also visible in Fig. 3, where the current intensity in the summer hemisphere is approximately three times stronger than that of the winter hemisphere. The Sq currents in the winter hemisphere are sometimes so weak that the "disappearance" of the current whorl occurs (e.g., Campbell et al. 1993; Rastogi 1993; Stening and Winch 2013).

Although the current intensity of the northern and southern Sq current systems changes annually, the total Sq current intensity $J_{\text {total }}$ changes semiannually with equinoctial peaks, as illustrated in Fig. 35 (from Yamazaki et al. 2010). Figure 36a shows month-to-month variations of $J_{\text {total }}$ derived from the empirical model of Yamazaki et al. (2011b). It can be seen that the semiannual variation in $J_{\text {total }}$ with equinoctial maxima is more pronounced under higher solar flux conditions. Figure 36b shows corresponding results from the NCAR TIE-GCM. The model well reproduces the semiannual variation of $J_{\text {total }}$ and its solar activity dependence. These results are obtained from the simulation that assumes constant geomagnetic activity $(K p=2)$. Therefore, the well-known semiannual variation of geomagnetic activity (e.g., Russell and McPherron 1973; Cliver et al. 2000) can be ruled out as the the main cause of the semiannual variation of the Sq currents. The TIE-GCM results suggest that the semiannual variation in $J_{\text {total }}$ is largely due to tidal waves from the lower atmosphere. In Fig. 36b, the red line indicates the results for the moderate solar activity condition $\left(F_{10.7}=150\right)$ calculated without the contribution of upward-propagating tides generated below $96 \mathrm{~km}$. The 
Sq ANNUAL AND SEMIANNUAL

AMPLITUDE VARIATIONS

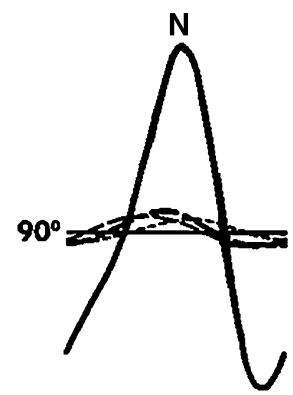

E

Z
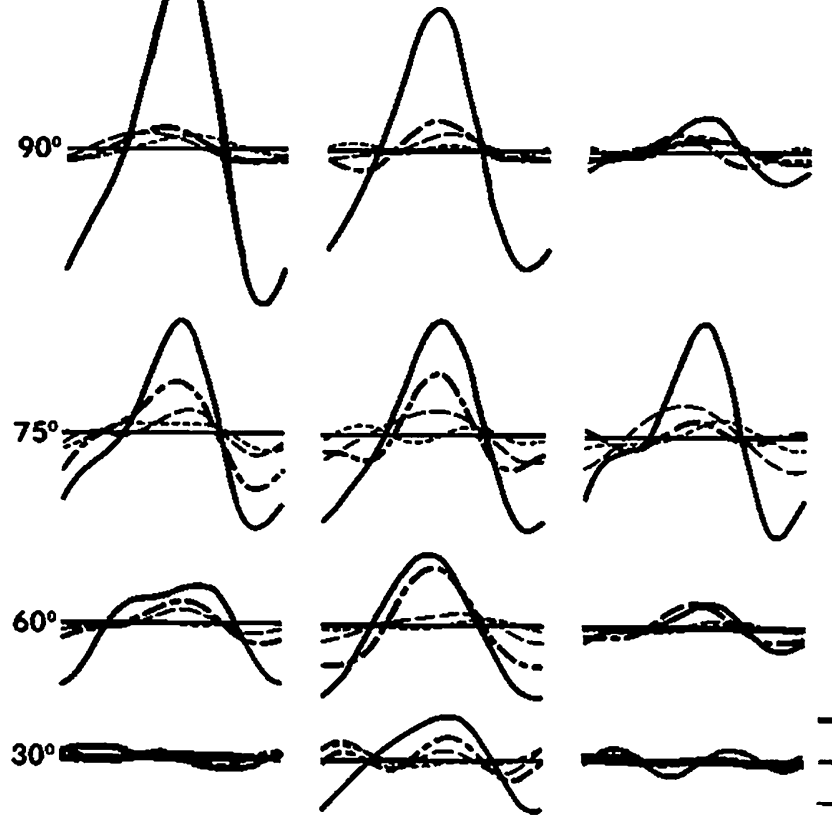

20
-16
-12
-8
-4
4
0
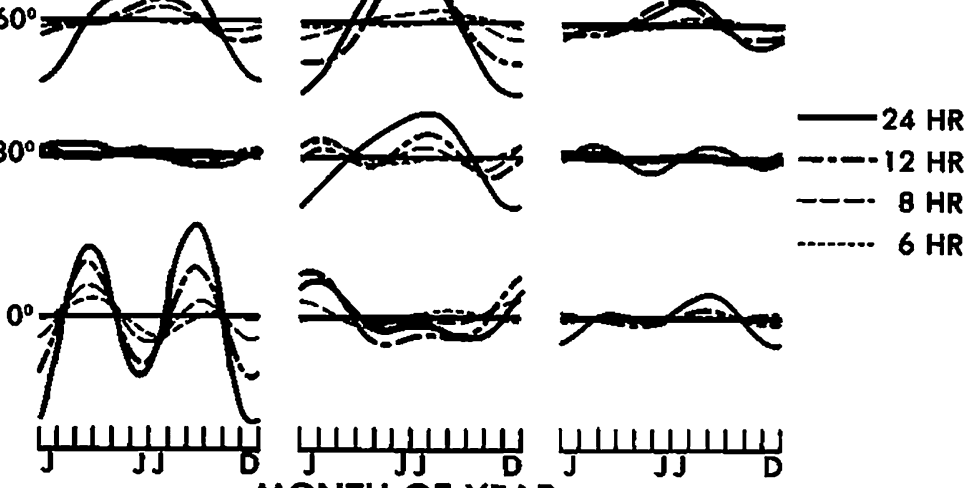

Fig. 34 Seasonal variations in the 24-, 12-, 8- and 6-hr Fourier components in the American sector for the year 1965. The results are shown for the magnetic-northward $(N)$, magnetic-eastward $(E)$, and vertical $(Z)$ components at $0^{\circ}, 30^{\circ}, 60^{\circ}, 75^{\circ}$ and $90^{\circ}$ north geomagnetic latitudes. From Campbell (1982)

semiannual variation of the Sq currents largely disappears when the effect of the upwardpropagating tides is removed.

The results of the empirical model in Fig. 36a also reveal an asymmetry in $J_{\text {total }}$ between the June and December solstices. The TIE-GCM does not capture this asymmetry (Fig. 36b). The discrepancy could be due to the inaccuracy of the upward-propagating tides in these simulations. Yamazaki et al. (2014b) discussed the importance of the upward-propagating tides in the simulation of geomagnetic daily variations.

The seasonal variation can also be found in the focus position of the Sq current system. As can be seen in Fig. 3, the northern Sq current focus is shifted to earlier local times during the summer (June) compared to the winter (December); likewise, the southern Sq current focus is shifted to earlier local times during the summer (December) compared to the winter (June). Figure 37 (after Yamazaki 2011) shows the latitudinal and local-time 
Fig. 35 Daily values of the total Sq current intensity $J_{\text {total }}$ in the East Asian sector from 2000 through 2002. The red line represents the 90-day running average. From Yamazaki et al. (2011a) (Color figure online)

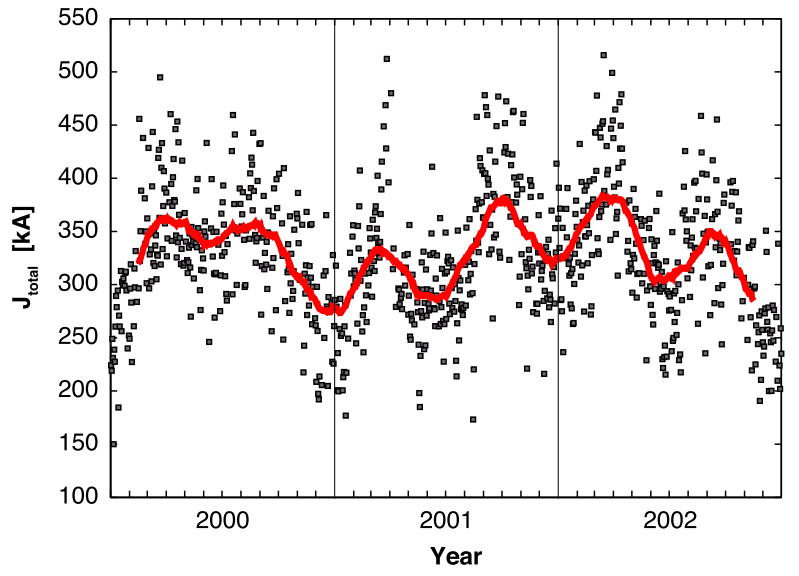

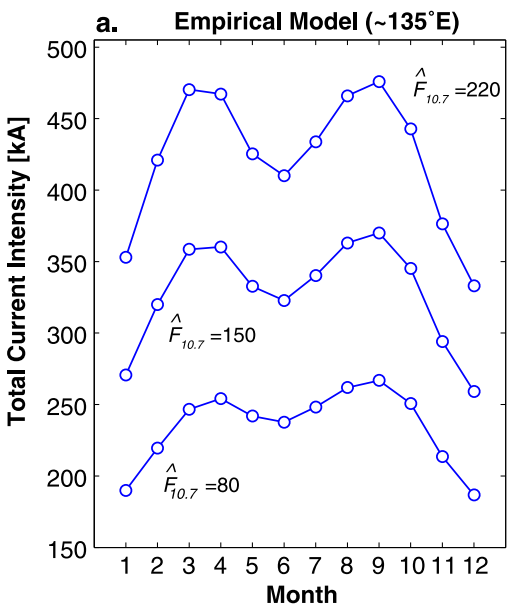

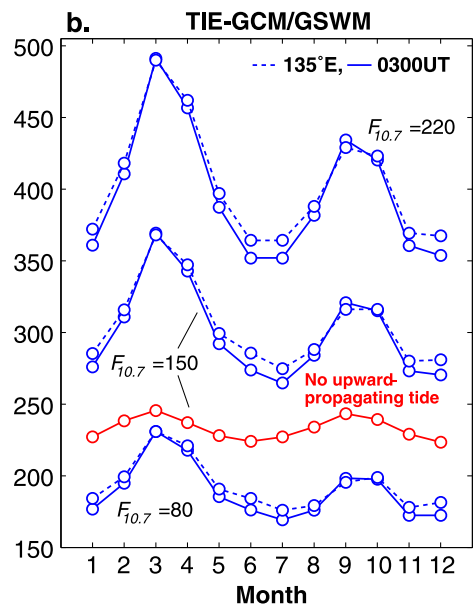

Fig. 36 (a) Month-to-month variations of the total Sq current intensity $J_{\text {total }}$ in the East Asian sector derived from the empirical model of Yamazaki et al. (2011b). The empirical model of Yamazaki et al. (2011b) is based on the slice method of the spherical harmonic analysis in the East Asian longitude zone $\left(\sim 135^{\circ} \mathrm{E}\right)$. The model is driven by the solar activity parameter $\hat{F}_{10.7}=\left\{F_{10.7}+\overline{F_{10.7}}\right\} / 2$, where $\overline{F_{10.7}}$ is the $F_{10.7}$ index averaged over the days from one to 81 days prior. (b) Month-to-month variations of $J_{\text {total }}$ simulated by the NCAR TIE-GCM. The dashed line indicates $J_{\text {total }}$ derived using the slice method of the spherical harmonic analysis at $135^{\circ} \mathrm{E}$ longitude, while the solid line indicates $J_{\text {total }}$ derived using the instantaneous method of the spherical harmonic analysis at $0300 \mathrm{UT}$. It is noted that there is no significant difference between the two results. The red line shows the results obtained from the simulation without upward-propagating tides from the lower atmosphere (Color figure online)

positions of the (red) northern and (blue) southern Sq current foci in the East Asian longitude sector during 2000-2002. The local time for the Sq current foci clearly shows an annual modulation. Similar results can be found in other longitude sectors, as shown in Fig. 38 (adopted from Campbell and Schiffmacher 1986, 1988b). Numerical studies have shown that inter-hemispheric field-aligned currents can cause such a local time shift in the equivalent Sq current system (e.g., Takeda 1990; Le Sager and Huang 2002a). In Figs. 37 and 38, the seasonal variation in the $\mathrm{Sq}$ focus latitude is much less clear compared to the seasonal 

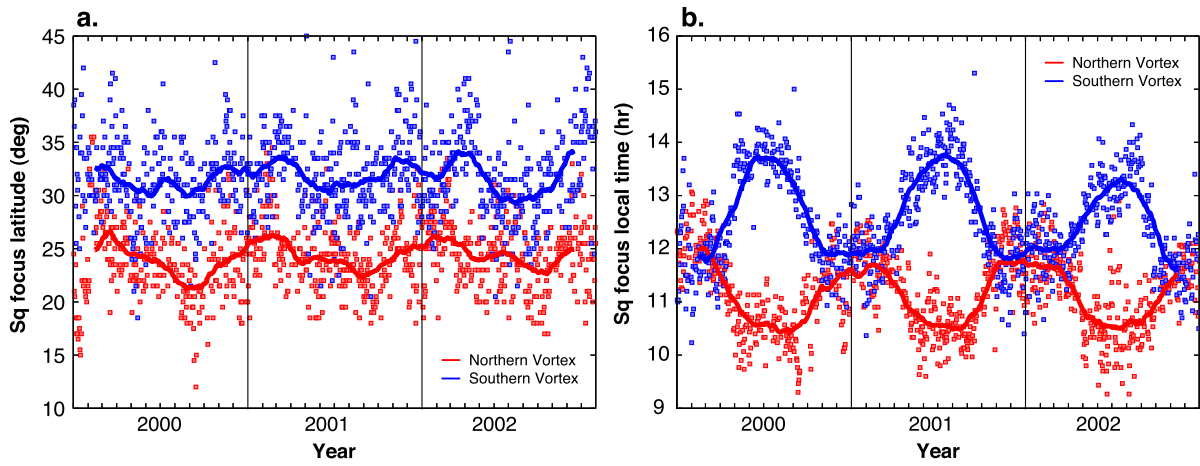

Fig. 37 Daily values of the Sq focus position in the East Asian sector from 2000 through 2002; (a) the magnetic dip latitude and (b) the local time of Sq foci. The solid lines represent the 90-day running average. From Yamazaki (2011)

a.

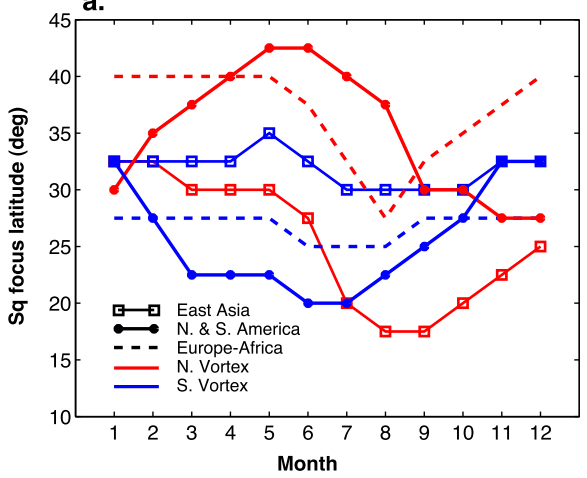

b.

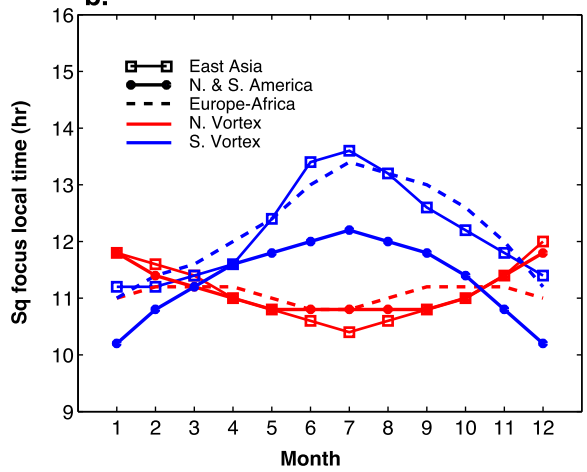

Fig. 38 Monthly values of the $\mathrm{Sq}$ focus position; (a) the geomagnetic latitude and (b) the local time of Sq foci. From Campbell and Schiffmacher (1986, 1988b)

variation in the Sq focus local time. Gupta (1973), examining the latitudinal movement of Sq foci during the solar maximum year of 1958, found that the northern and southern Sq foci move together from month to month. That is, when the northern focus moves northward, the southern focus also moves northward. The results in Fig. 37a confirm Gupta's fining. As shown in Fig. 32, the seasonal variation in the Sq focus latitude is more evident during solar minimum than solar maximum.

\subsubsection{EEJ}

It is known for a long time that the equatorial electrojet shows a prominent semiannual variation (e.g., Chapman and Raja Rao 1965; Yacob 1966; Bhargava et al. 1973). The amplitude of the semiannual variation of EEJ is much greater than that expected from the seasonal change in the ionospheric conductivity. Thus, neutral winds are thought to play a role (Campbell 1981; Stening 1991). The semiannual variation of EEJ is evident only during morning hours and it practically disappears in the afternoon, which is illustrated in Fig. 39 (after Rastogi et al. 1994). Recently, Yamazaki et al. (2014b) conducted TIE-GCM simulations, successfully reproducing the semiannual variation in the magnetic-northward $(N)$ 
Fig. 39 Seasonal variations in the $H$-component geomagnetic field at Trivandrum $\left(8.5^{\circ} \mathrm{N}\right.$, $\left.77.0^{\circ} \mathrm{E}\right)$ at different local times for (left) high solar activity periods of 1979-1981 and (right) low solar activity periods of 1984-1986. From Rastogi et al. (1994)

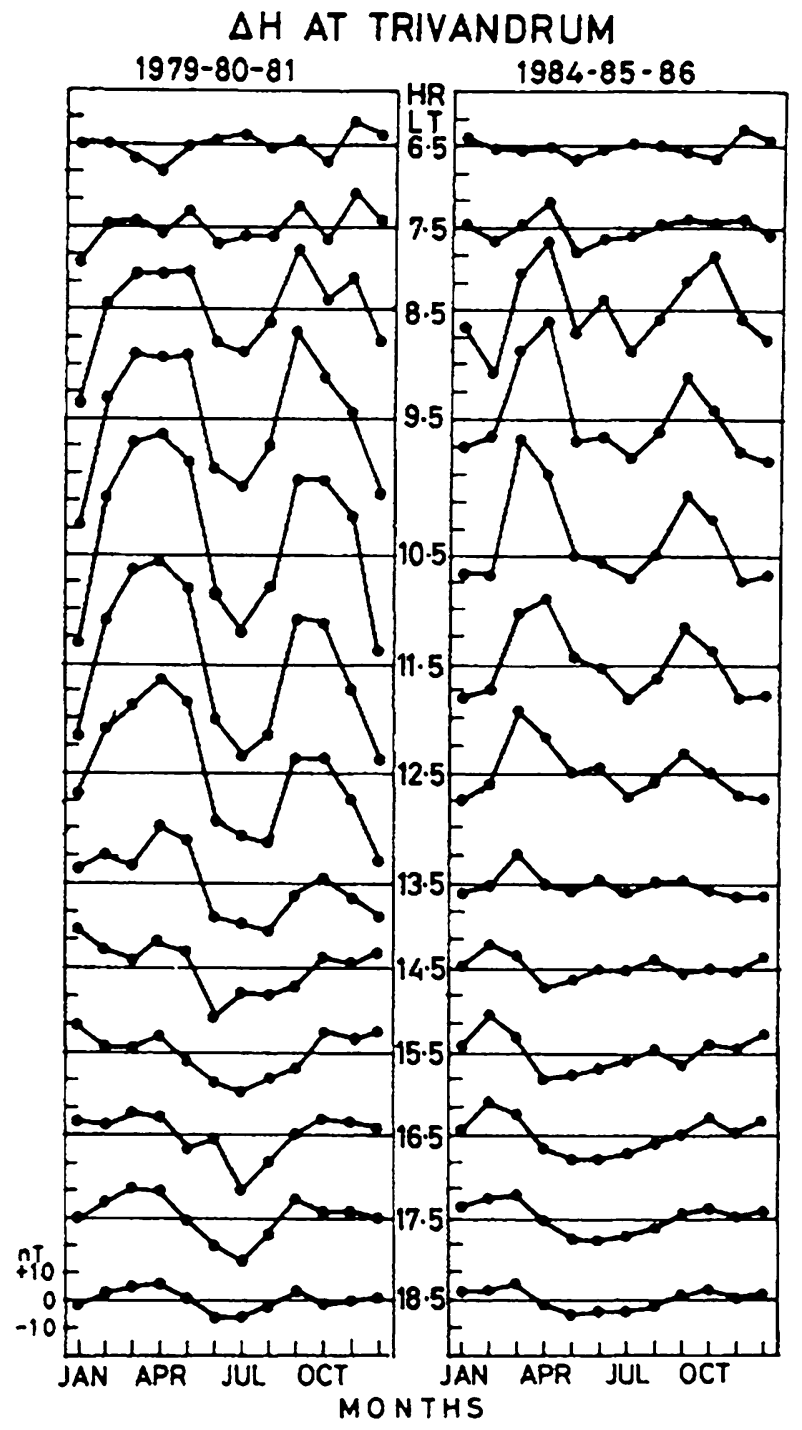

component of the geomagnetic field near the magnetic equator. In Fig. 40 (from Yamazaki et al. 2014b), the top panels show observed and simulated ground-level geomagnetic perturbations $\Delta N$ at Tirunelveli $\left(8.7^{\circ} \mathrm{N}, 77.8^{\circ} \mathrm{E}\right)$, India for the year 2008 . The model well captures the semiannual variation in $\Delta N$ that is most significant at 10-11 LT. In the bottom panels, the simulated $\Delta N$ is divided into two components: (left) $\Delta N$ due to upwardpropagating tides from the lower atmosphere and (right) $\Delta N$ due to the tides generated in situ by solar EUV heating. It can be seen that the semiannual variation in $\Delta N$ is mainly due to upward-propagating tides from the lower atmosphere. The locally generated tides also contribute to the semiannual variation in $\Delta N$, but to a less extent. The semiannual variation in $\Delta N$ produced by locally generated tides reinforces the semiannual variation due to the upward propagating tides in the morning, while they tend to cancel each other in 

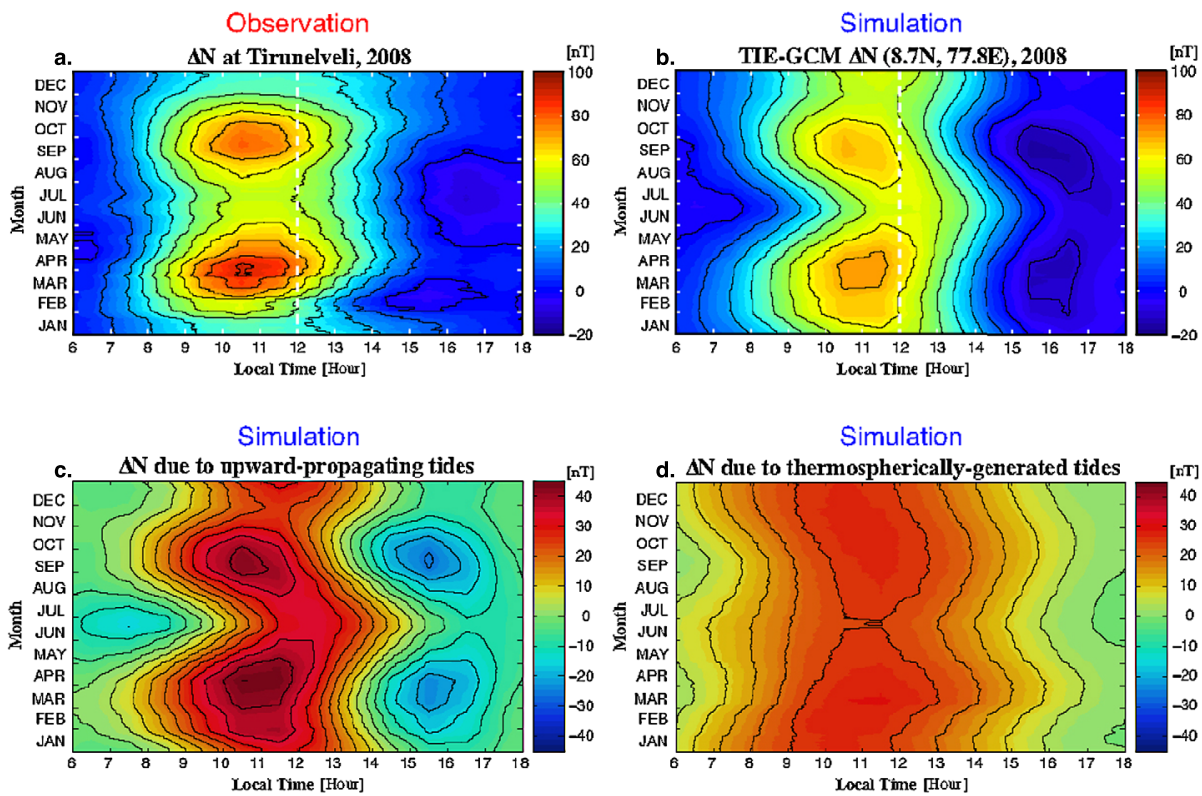

Fig. 40 Contour plots for seasonal and local-time variations in the magnetic-northward $(N)$ component of the geomagnetic daily variation at Tirunelveli $\left(8.7^{\circ} \mathrm{N}, 77.8^{\circ} \mathrm{E}\right)$ for 2008 . (a) Observations. (b) NCAR TIE-GCM simulation results. (c) NCAR TIE-GCM simulation results; the contribution of upward-propagating tides from the lower atmosphere. (d) NCAR TIE-GCM simulation results; the contribution of tides locally generated in the thermosphere by in-situ solar heating. From Yamazaki et al. (2014b)

the afternoon. This explains why the semiannual variation is present only during morning hours.

\subsubsection{L}

Figure 41 (from Matsushita and Xu 1984) shows the seasonal change in the L current system. Similar to the Sq current system, the current intensity tends to be greater in the summer hemisphere than in the winter hemisphere. The total current intensity is largest during the Northern Hemisphere winter. The lunar modulation of the equatorial electrojet is also greatest during the northern winter, except in the West Pacific region (Rastogi and Trivedi 1970; Stening 2011). The strong L currents during the northern winter is probably contributed by the big $\mathrm{L}$ days in which the amplitude of $\mathrm{L}$ is several times larger than other normal days ((Bartels and Johnston 1940); Onwumechili 1964). Recent studies have shown that the big $\mathrm{L}$ days are often associated with the occurrence of stratospheric sudden warmings (Fejer et al. 2010; Yamazaki 2014). A stratospheric sudden warming is a large-scale disturbance in the middle and lower atmosphere, which usually occurs during the northern winter. It has been shown that lunar atmospheric tides are subject to an amplification during stratospheric sudden warming events, which leads to enhanced lunar tidal effects in the ionosphere (Stening et al. 1997; Pedatella et al. 2012b; Forbes and Zhang 2012). The effect of stratospheric sudden warmings on the ionospheric dynamo will be discussed in more detail in Sect. 4.3.3. A study by Pedatella (2014) showed that atmospheric lunar tides are strongest during Northern Hemisphere winter months even without a stratospheric sudden warming; 

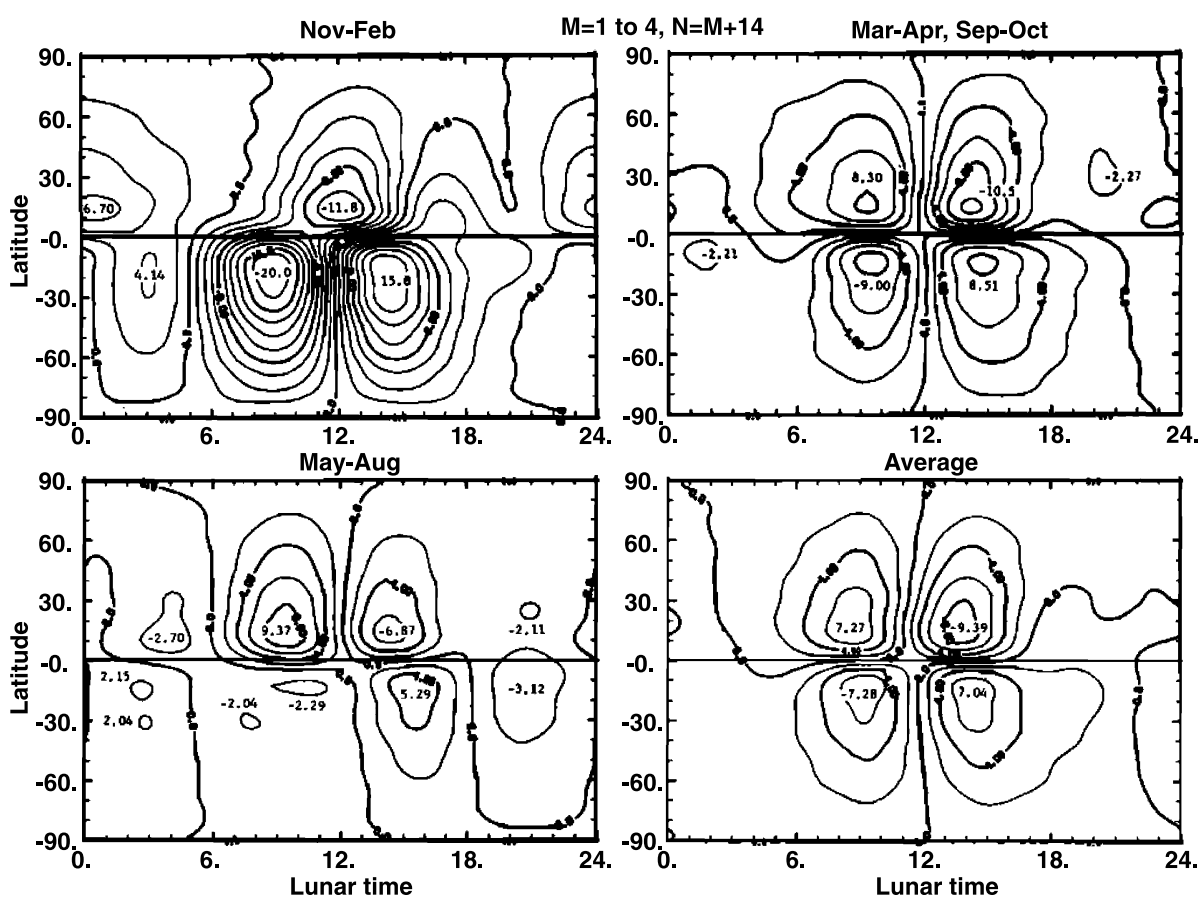

Fig. 41 External equivalent L current systems during July 1957-December 1959. The contour intervals are 2 kA. From Matsushita and Xu (1984)

thus, the strong lunar currents observed around the December solstice may not be solely due to stratospheric sudden warming events.

\subsection{Day-to-Day Variation}

\subsubsection{Irregular Changes}

The amplitude and phase of the Sq variation show significant day-to-day changes. The dayto-day variation of Sq is often "random-like", and the mechanism is not well understood. The correlation of Sq at a pair of stations decreases with increasing distance between the two stations, reaching 0.5 for separations of about $40^{\circ}$ in longitude and $15^{\circ}$ in latitude (Schlapp 1968; Greener and Schlapp 1979). In order to illustrate the nature of the day-to-day variability of Sq, we have analyzed the horizontal-component $(H)$ geomagnetic field obtained from the Indian longitude sector. The stations used are: Tirunelveli (TIR, 8. $7^{\circ} \mathrm{N}, 77.8^{\circ} \mathrm{E}$ ), Alibag $\left(\mathrm{ABG}, 18.6^{\circ} \mathrm{N}, 72.9^{\circ} \mathrm{E}\right)$, Jaipur (JAI, 26.9 $\left.{ }^{\circ} \mathrm{N}, 75.8^{\circ} \mathrm{E}\right)$, Kashi $\left(\mathrm{KSH}, 39.5^{\circ} \mathrm{N}, 76.0^{\circ} \mathrm{E}\right)$, Novosibirsk (NVS, 55.0 $\mathrm{N}, 82.9^{\circ} \mathrm{E}$ ); see Fig. 42 (left). The Sq variation $\Delta H$ was determined for each station using the Dst correction technique, described in Sect. 3.3. The middle panel of Fig. 42 shows the average daily variations for March and April, 2009. A large amplitude $\Delta H$ at TIR represents the effect of the equatorial electrojet. $\Delta H$ at ABG and JAI are dominated by the eastward Sq currents, while $\Delta H$ at NVS is dominated by the westward Sq currents. $\Delta H$ is relatively small at KSH because it is located near the Sq focus. The noontime values of $\Delta H$ are plotted in the right panel of Fig. 42 for March-April, 2009, showing highly irregular day-to-day changes at each station. Table 5 displays correlation coefficients 
a.

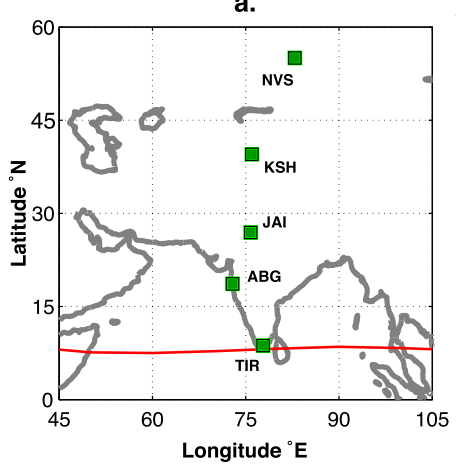

b.

$\Delta H($ av. Mar-Apr 2009)

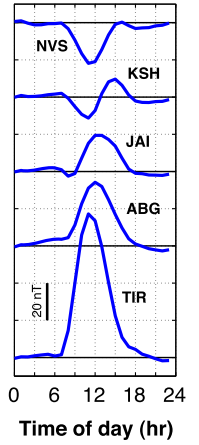

c.

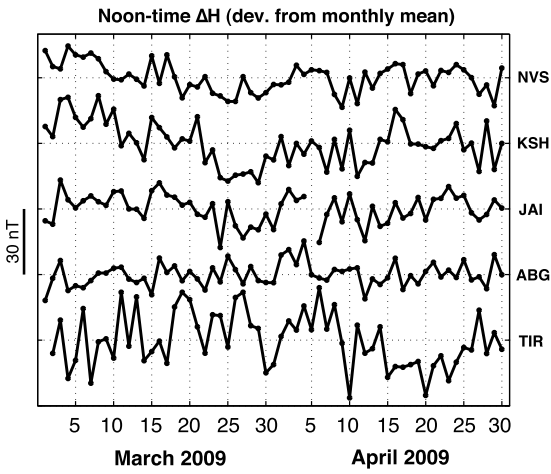

Fig. 42 (a) A map of stations in the Indian sector. (b) Average daily variations of the geomagnetic field in the $H$ component during March-April 2009. (c) Day-to-day variations in the noon-time $H$ field during March-April 2009

Table 5 Correlation coefficient $R$ for the noon-time $\Delta H$ during March-April, 2009

Significant $(>95 \%)$ results are indicated by bold font

\begin{tabular}{lrrrrrrr}
\hline & TIR & ABG & JAI & KSH & NVS & $F_{10.7}$ & \multicolumn{1}{c}{$A p$} \\
\hline TIR & & & & & & -0.24 & 0.02 \\
ABG & 0.17 & & & & & 0.18 & -0.11 \\
JAI & -0.15 & $\mathbf{0 . 4 7}$ & & & & -0.13 & -0.09 \\
KSH & $\mathbf{- 0 . 3 1}$ & -0.15 & $\mathbf{0 . 4 9}$ & & & $\mathbf{0 . 2 9}$ & -0.03 \\
NVS & $\mathbf{- 0 . 3 5}$ & $\mathbf{- 0 . 3 4}$ & 0.24 & $\mathbf{0 . 6 8}$ & & -0.19 & -0.23 \\
\hline
\end{tabular}

for the noon-time $\Delta H$ evaluated for different pairs of stations. As expected, the correlation tends to be higher for a closer pair of the stations. An exception for this is the results for TIR. $\Delta H$ at TIR does not correlate with $\Delta H$ at the nearest station ABG but it negatively correlates with $\Delta H$ at KSH and NVS, indicating the linkage of the equatorial electrojet with the global Sq current system. Table 5 also shows the correlation with solar activity index $F_{10.7}$ and geomagnetic activity index $A p$. The correlation is poor in either case.

The day-to-day variation in the equivalent Sq current system has been studied by many researchers (Hasegawa 1960; Suzuki 1978, 1979; Takeda 1984; Takeda and Araki 1984a; Stening 2008; Yamazaki 2011). As is clear from Figs. 35 and 37, both the intensity and shape of the Sq current system change from day to day. Similarly, the EEJ intensity shows significant day-to-day variations (e.g., Fambitakoye and Mayaud 1976a; Onwumechili 1998; Stening et al. 2005b). Since the day-to-day variability exists even when disturbances associated with solar and geomagnetic activity are absent, neutral winds are often thought to be responsible for the short-term variability of Sq and EEJ. Miyahara and Ooishi (1997) were the first to investigate the effect of variable neutral winds on the ionospheric dynamo by means of a numerical simulation. They used a thin-shell dynamo model, driven by winds from a general circulation model of the middle atmosphere. They found that the waves from below the ionosphere make a significant contribution to the day-to-day variability of the dynamo-region currents and ground-level Sq variations. Later studies, using improved dynamo models with more realistic neutral winds, have also underscored the importance of lower-atmospheric drivers in the short-term variability of the ionospheric dynamo (Kawano-Sasaki and Miyahara 2008; Jin et al. 2011; Liu et al. 2013; Fang et al. 2013; 

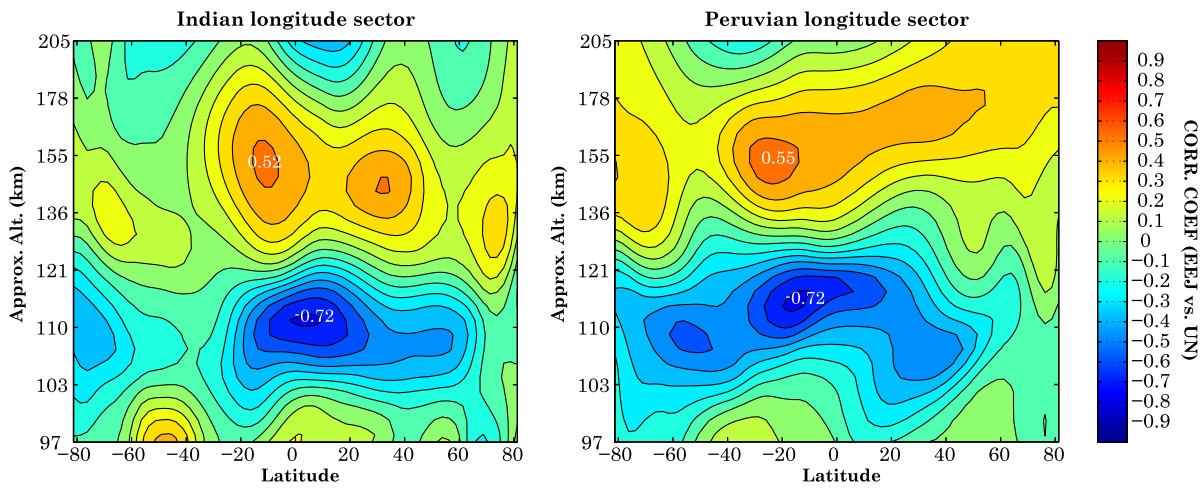

Fig. 43 The distribution of correlation coefficients between the noon-time eastward wind and peak equatorial electrojet density as a function of height and latitude for the (left) Indian sector and (right) Peruvian sector during May-June 2009. From Yamazaki et al. (2014a)

Yamazaki et al. 2014a). Among those studies, Yamazaki et al. (2014a) attempted to clarify where the day-to-day variability of the equatorial electrojet is produced. They compared the current density of EEJ with neutral wind velocities at various latitude, longitude, and height. The left panel of Fig. 43 (after Yamazaki et al. 2014a) shows the correlation between the noon-time EEJ and eastward wind velocity in the Indian sector. The most significant correlation $(R=-0.72)$ is found at $\sim 110 \mathrm{~km}$ near the magnetic equator. The negative correlation indicates that the eastward and westward winds in this region tend to decrease and increase the eastward flow of EEJ, respectively. The results for the Peruvian sector (Fig. 43, right) are largely consistent with the results from the Indian sector. The relationship between variable neutral winds and Sq current intensity is yet to be studied.

\subsubsection{Planetary Wave Effect}

Geomagnetic signatures of Sq and EEJ sometimes show periodic oscillations. The oscillations with periods close to 2 days, 5 days, 10 days, and 16 days are often considered to be due to planetary waves or Rossby waves that have been observed in the lower and middle atmosphere. Those planetary waves represent "normal mode" or "resonant" oscillations of the atmosphere (e.g., Salby 1984). Global climatology of 2-, 5-, 10- and 16-day waves has been established by satellite measurements, e.g., Gu et al. (2013), Moudden and Forbes (2014) for 2-day waves; Wu et al. (1994), Riggin et al. (2006) for 5-day waves; Forbes and Zhang (2015) for 10-day waves; and McDonald et al. (2011), Day et al. (2011) for 16-day waves. If the amplitude of the planetary waves is sufficiently large in the dynamo region, they will drive ionospheric currents and affect geomagnetic perturbations on the ground. Even if the planetary waves dissipate before they reach the dynamo region, they can still interact with tides and mean flow in the middle atmosphere, which will affect the upward propagation of tides to the dynamo region, and thus affect the ionospheric dynamo (e.g., Liu et al. 2010; Chang et al. 2011). Either case, the presence of the planetary waves would lead to oscillations of $\mathrm{Sq}$ and EEJ with periods similar to those of the waves. Previous studies have attempted to identify the effect of the planetary waves in geomagnetic data (see Table 6). A spectral analysis is generally used to find geomagnetic field oscillations having periods near 2, 5, 10 and 16 days. Caution is required for the interpretation of the spectral analysis results, because 2-, 5-, 10-, and 16-day periodicities may exist in solar and geomagnetic activity as well. Indices of solar and geomagnetic activity often 


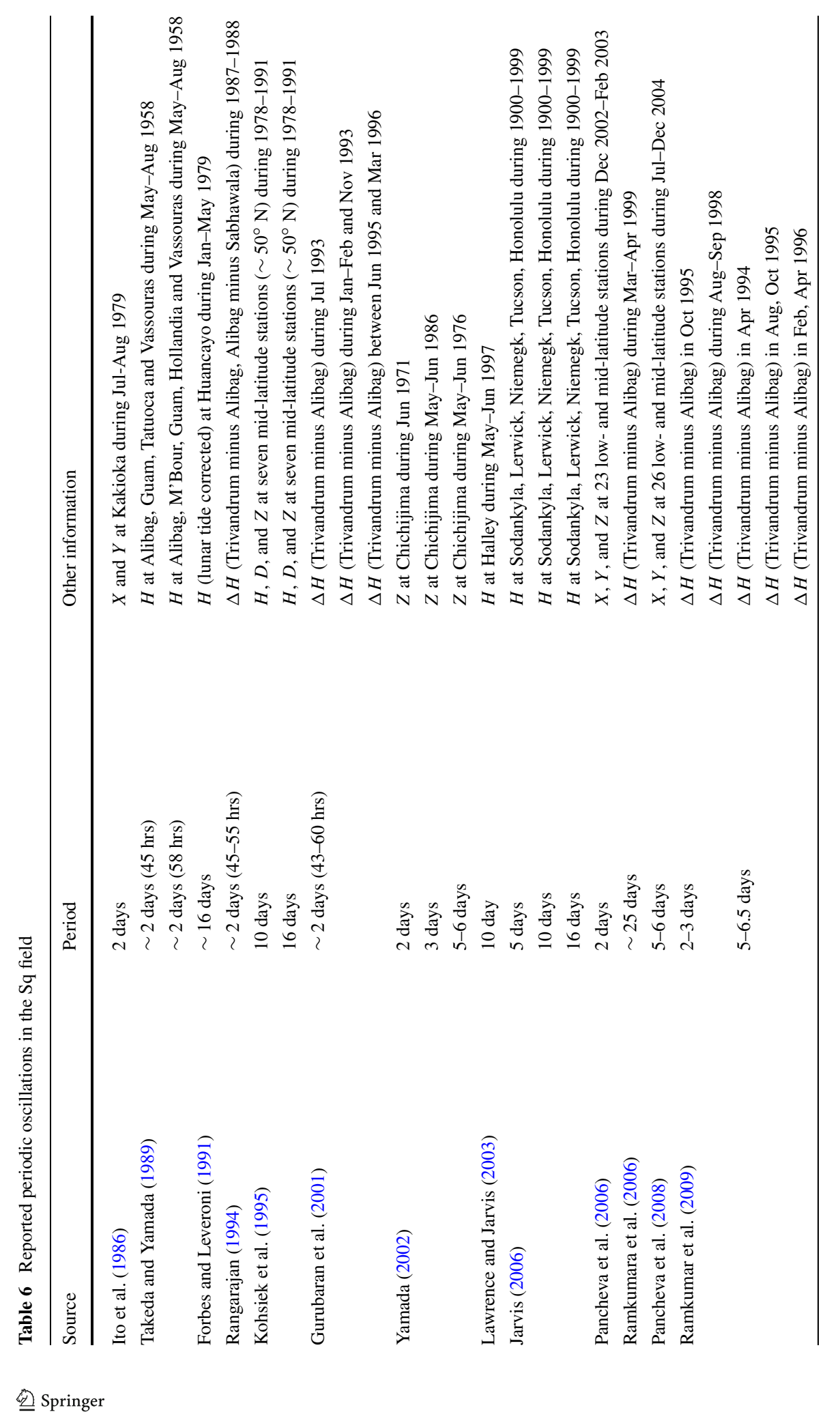




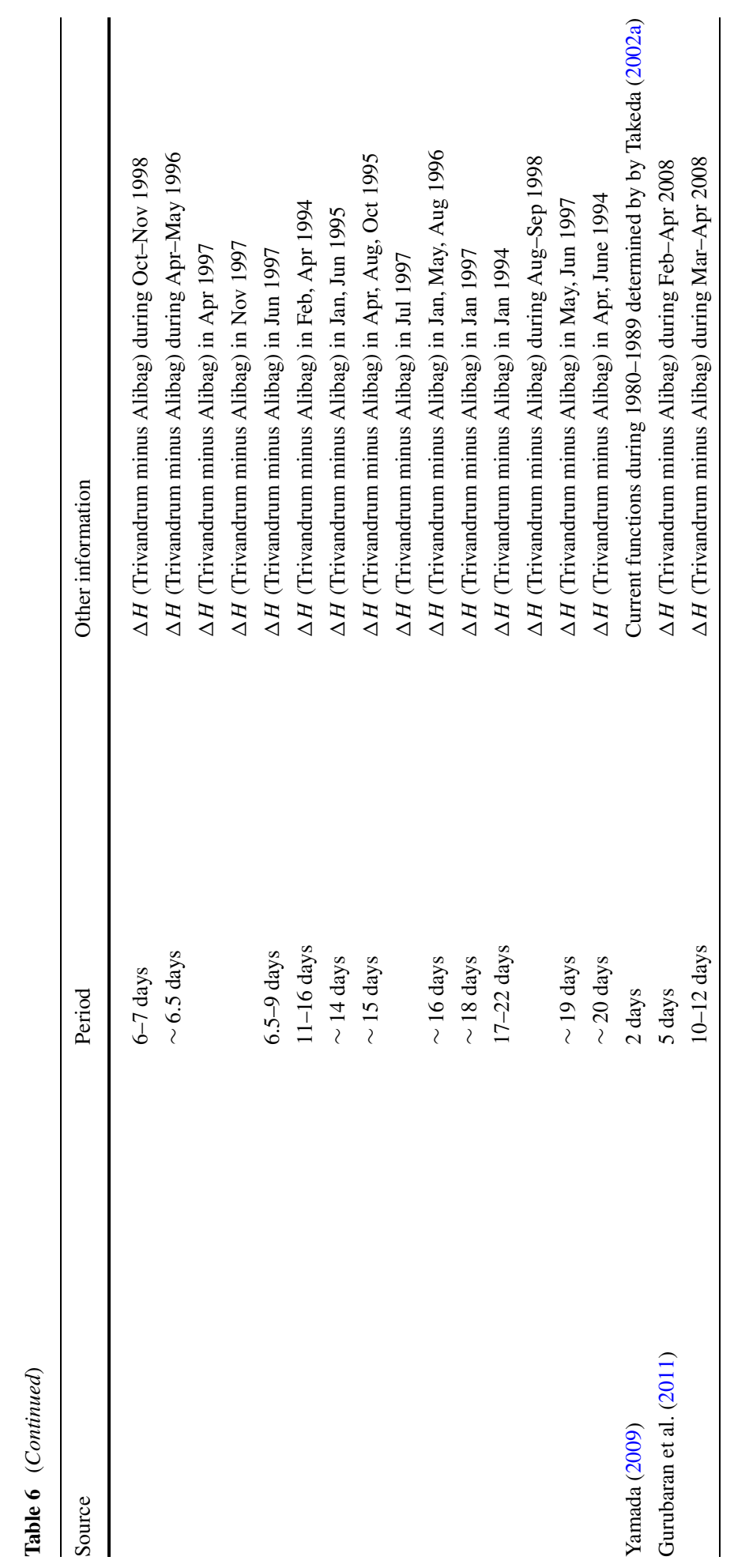


a.

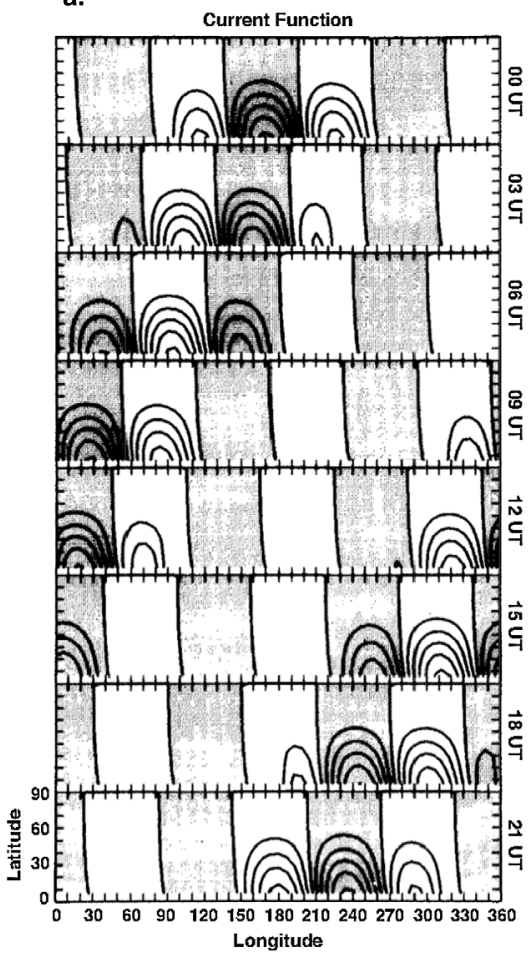

b.
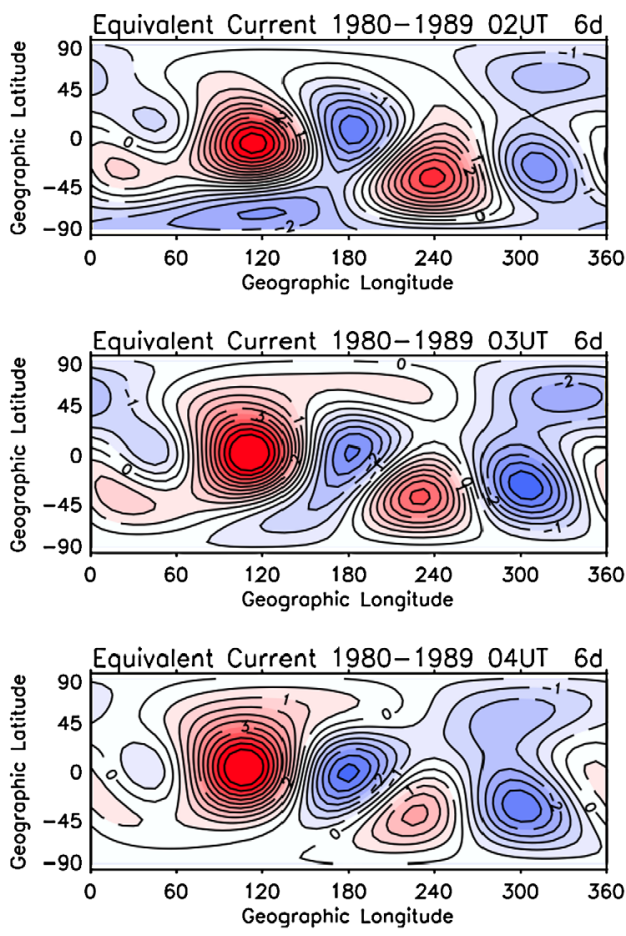

Fig. 44 (a) Simulated equivalent current functions for the 2-day geomagnetic variation with contour intervals of $10 \mathrm{kA}$, from Ito et al. (1986). (b) Observed equivalent current functions corresponding to the 2-day geomagnetic variation with contour intervals of $0.5 \mathrm{kA}$, from Yamada (2009)

show the 27-day solar rotation cycle and its subharmonics (e.g., $\sim 13.5$ days and $\sim 9$ days). These periodicities are sometimes found in Sq and EEJ data (e.g., Briggs 1984; Uozumi et al. 2008). Also, the effect of the semi-monthly lunar tide (=14.77 days) can easily alias into the 16-day spectrum, especially when the amplitude and phase of the lunar tide are changing with time, such as during stratospheric sudden warming events. Furthermore, Gasperini and Forbes (2014) showed that the combined effect of solar rotation ( $=27$ days) and lunar tide ( $=14.77$ days) can cause sideband frequencies corresponding to 9.55 - and 32.61-day periods. Love and Rigler (2014) also found various spectral components within the 2-16 day range that arise from solar and lunar tides and intermodulations among them.

Figure 44 illustrates global ionospheric current systems associated with the 2-day oscillation of the Sq field, from (a) numerical simulation by Ito et al. (1986) and (b) data analysis by Yamada (2009). The contour intervals are $10 \mathrm{kA}$ and $0.5 \mathrm{kA}$ in (a) and (b), respectively. Thus, the current intensity is much stronger in the calculation by Ito et al. (1986) compared to the climatological average derived by Yamada (2009). Takeda and Yamada (1989) also calculated ionospheric currents using the same wind model as Ito et al. (1986) but using a more realistic dynamo model. Their results showed a current pattern very similar to that by Ito et al. (1986) but with much smaller current intensity ( $\pm 4 \mathrm{kA}$ ), comparable to the results by Yamada (2009). In Fig. 44, the current pattern is consistent between the simulated and observed results. There are six current vortices in the longitudinal direction near the equator, which travel westward. The dayside vortices are much enhanced in intensity because of 
elevated ionospheric conductivities during the daytime. The longitudinal structure is owing to the wind pattern of the 2-day wave, which is dominated by a westward-propagating mode with zonal wave number 3. In contrast to Yamada's results, Pancheva et al. (2006) found westward propagating waves with zonal wavenumber 1 and 2 in geomagnetic data during the 2-day wave event of December 2002-February 2003. More work is necessary to establish the 2-day wave effect on the global ionospheric current system. The spatial structures of the ionospheric current system for the 5-, 10-, and 16-day waves have been much less studied, which also requires future work.

\subsubsection{Stratospheric Sudden Warming Effect}

A stratospheric sudden warming is a large-scale disturbance in the middle atmosphere, which is caused by the interaction between quasi-stationary planetary waves and the zonal mean flow (Andrews et al. 1987). An event usually takes place in the Northern Hemisphere at high latitudes during the winter time, where planetary-wave activity is high. A stratospheric sudden warming event is characterized by a rapid increase in the polar temperature and a weakening (or reversal) of the polar vortex. In practical terms, the detection of a stratospheric sudden warming event relies on the daily mean values of the polar stratospheric temperature at $10 \mathrm{hPa}(\sim 32 \mathrm{~km}$ height $)$ and zonal mean zonal wind at $60^{\circ} \mathrm{N}$ at $10 \mathrm{hPa}$. Especially, when the zonal mean zonal wind at this latitude and height. is reversed from the normal eastward flow to westward, the event is called a "major warming" and other events are called "minor warmings". The effect of stratospheric sudden warming is known to extend well into the thermosphere (e.g., Liu and Roble 2002; Yamazaki et al. 2015).

Early studies hypothesized effects of stratospheric sudden warmings on the ionospheric dynamo (e.g., Brown and Williams 1969; Stening 1977d; Matsushita and Xu 1984; Stening et al. 1996; Rastogi 1999). In particular, Stening (1977d) and Stening et al. (1996) found that winter-time counter electrojet events tend to occur during stratospheric sudden warmings. Vineeth et al. (2009) confirmed this correlation, and also pointed out that the counter electrojet during stratospheric sudden warmings is prone to a quasi 16-day modulation. Sridharan et al. (2009) demonstrated that there is an enhancement in the amplitude of the semidiurnal tide in the mesosphere during counter electrojet events associated with stratospheric sudden warmings. Fejer et al. (2010) recognized the following characteristics of the counter electrojet events during stratospheric sudden warmings: (1) the onset of the afternoon counter electrojet tends to occur near new moon or full moon; (2) the afternoon counter electrojet is often accompanied by an enhanced eastward flow in the morning; and (3) the time for the maximum eastward or westward currents shifts to later local times on succeeding days. All these features are consistent with the effect of lunar currents. Thus, Fejer et al. (2010) suggested that strongly enhanced atmospheric lunar tides drive electrodynamic perturbations during stratospheric sudden warmings. Figure 45 (from Yamazaki 2014) shows an example of the afternoon counter electrojet observed during the January2009 stratospheric sudden warming event, illustrating the characteristic behaviors of the afternoon counter electrojet described above.

Numerical studies have shown that an amplification occurs in both solar and lunar semidiurnal tides at dynamo-region heights in response to stratospheric sudden warmings (Pedatella et al. 2012b, 2014a; Pedatella and Liu 2013). Those tidal changes can result from changes in the mean flow (e.g., Stening et al. 1997), interaction with planetary waves (e.g., Liu et al. 2010; Maute et al. 2014), and changes in the tidal sources (e.g., Goncharenko et al. 2012). Besides, Forbes and Zhang (2012) suggested that a change in the resonance property of the atmosphere during stratospheric sudden warming events leads to a significant increase in the amplitude of semidiurnal lunar tides. 
Fig. 45 The response of the equatorial electrojet to the January 2009 stratospheric sudden warming event. (a) The temperature at the North Pole at $10 \mathrm{hPa}$ ( $32 \mathrm{~km}$ height). The blue line shows the daily values for December 2008-February 2009, while the magenta line represents the climatological average. (b) The difference in the $H$-component geomagnetic field at an equatorial station, Ponape $\left(7.0^{\circ} \mathrm{N}, 158.3^{\circ} \mathrm{E}\right)$, and off-equatorial station, Guam $\left(13.6^{\circ} \mathrm{N}, 144.9^{\circ} \mathrm{E}\right)$. From Yamazaki (2014) (Color figure online)

\section{a.}
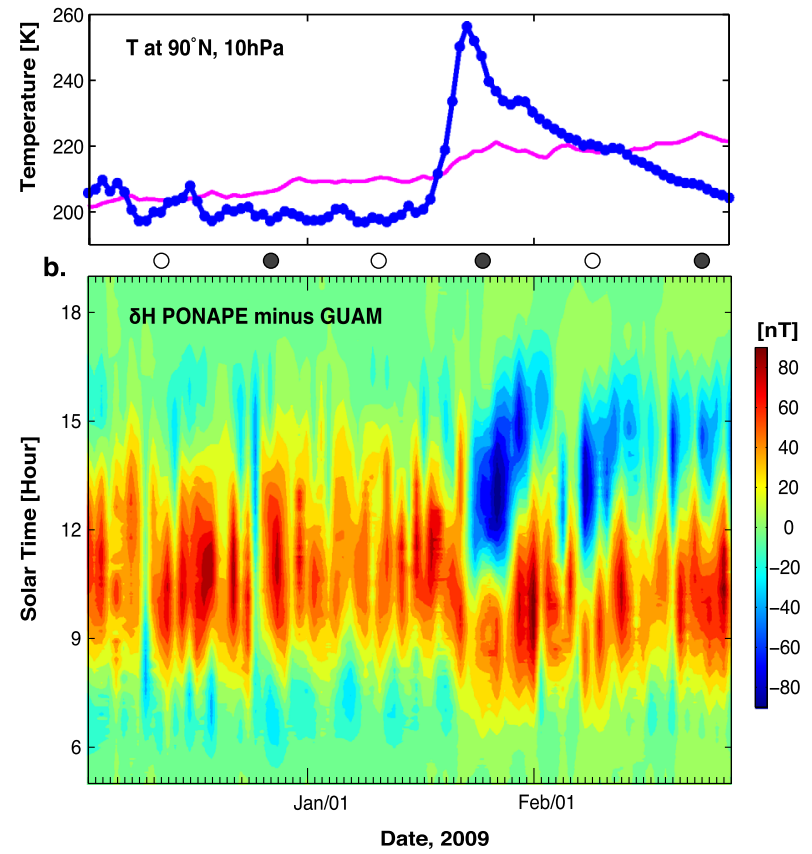

Park et al. (2012), analyzing magnetic data from the CHAMP satellite during 20012009 , found a one-to-one correspondence between the lunar modulation of the equatorial electrojet and the occurrence of stratospheric sudden warmings. Yamazaki et al. (2012b), using long-term ground measurements, showed that the correlation between the amplification of geomagnetic lunar variations and the occurrence of stratospheric sudden warmings can be traced back as early as 1958 . They noted that there are some years when a lunar tidal amplification occurs without a stratospheric sudden warming event and other years when there is no lunar tidal amplification during a stratospheric sudden warming event. Stening (2011), analyzing equatorial data during 1960-2009, also noted that not all stratospheric sudden warmings cause a counter electrojet event. A numerical study by Pedatella and Liu (2013) showed that the lunar tidal amplification depends on the phase of the moon relative to the timing of the stratospheric sudden warming. Thus, the magnitude of the lunar tidal amplification is not solely dependent on the magnitude of the stratospheric sudden warming. Siddiqui et al. (2015a) extended the analysis of geomagnetic data to the periods when there was no direct observations of stratospheric sudden warmings. From the occurrence frequency of large-amplitude L variations, they suggested that there were approximately six stratospheric sudden warming events per decade during 1926-1951.

The timing and magnitude of the lunar tidal enhancement is related to those of the polar vortex weakening defined at $1 \mathrm{hPa}(\sim 48 \mathrm{~km}$ ) (Zhang and Forbes 2014a, 2014b; Chau et al. 2015). In Fig. 46 (from Siddiqui et al. 2015b), the power of lunar tidal wave in the $H$ component geomagnetic field at Huancayo $\left(12.0^{\circ} \mathrm{S}, 75.3^{\circ} \mathrm{W}\right)$ is plotted for the years $1997-$ 2013. It can be seen that the lunar tidal amplification occurs near the polar vortex weakening indicated by the red lines.

Electrodynamic effects during stratospheric sudden warmings are not limited to the equatorial region but rather global. Figures 47a and 47b (from Yamazaki 2014) show equiva- 


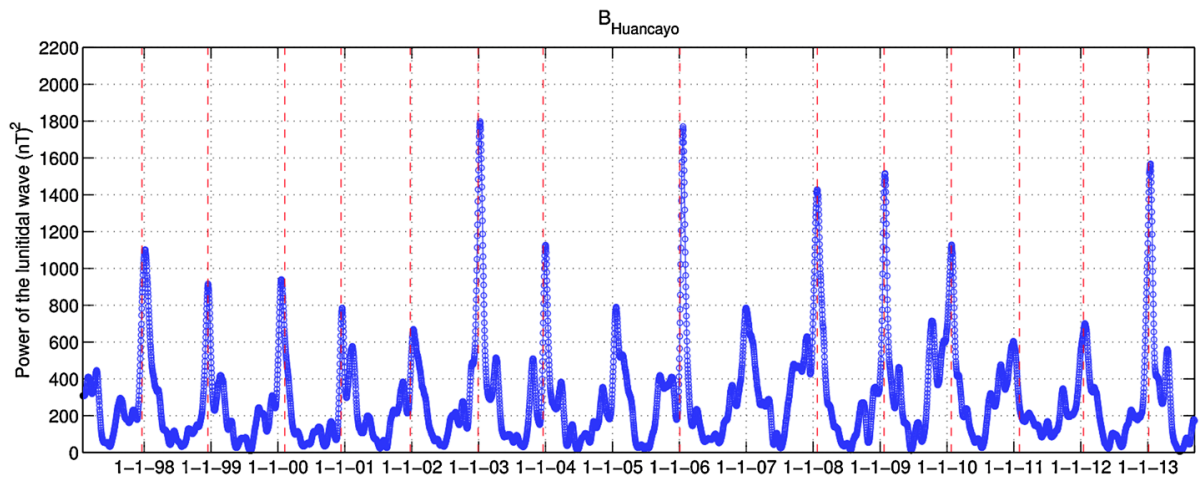

Fig. 46 The power of the lunar tidal wave in $H$ at Huancayo $\left(12.0^{\circ} \mathrm{S}, 75.3^{\circ} \mathrm{W}\right)$ during $1997-2013$. The vertical red lines denote the days for the peak polar vortex weakening. From Siddiqui et al. (2015b) (Color figure online)
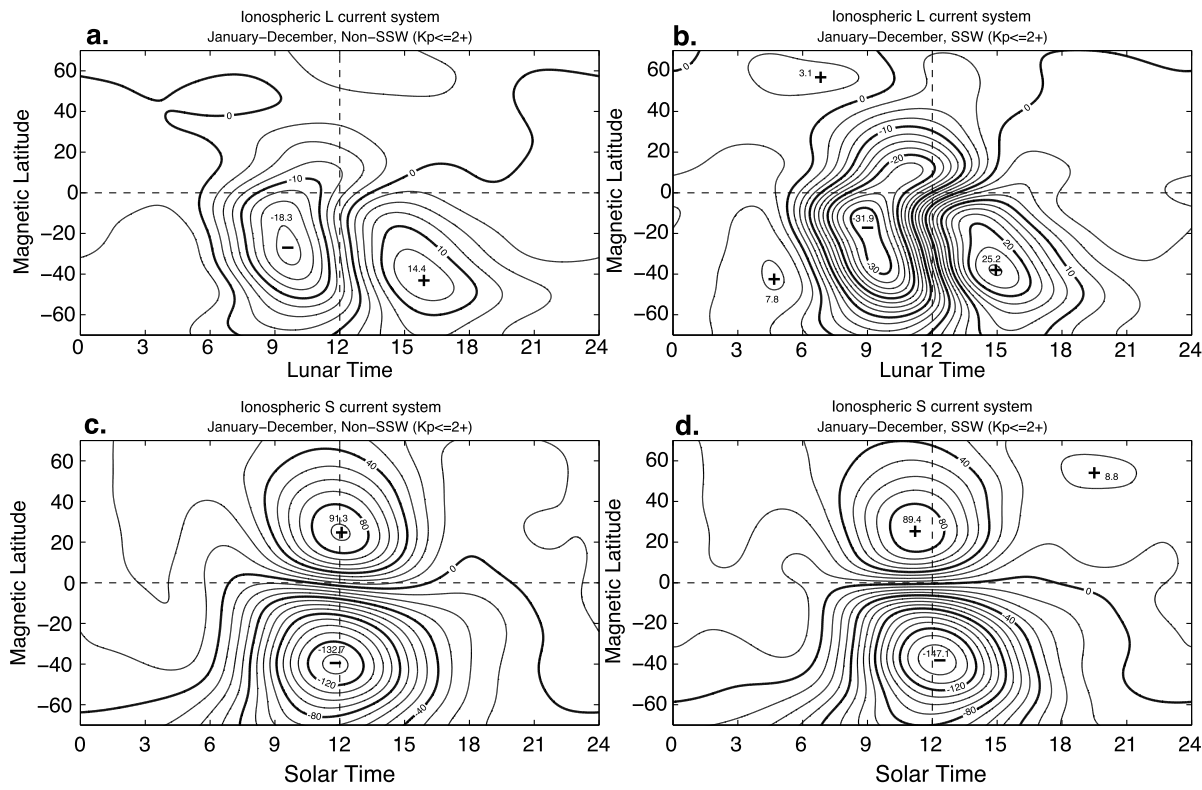

Fig. 47 (a) The equivalent lunar current system at new moon, averaged over the winters without a stratospheric sudden warming during 1958-2007. (b) The equivalent lunar current system at new moon, averaged over stratospheric sudden warming events during 1958-2007. (c) The equivalent solar current system averaged over the winters without a stratospheric sudden warming during 1958-2007. (d) The equivalent solar current system averaged over stratospheric sudden warming events during 1958-2007. The contour intervals are $2.5 \mathrm{kA}$ for the lunar currents and $10 \mathrm{kA}$ for the solar currents. From Yamazaki (2014)

lent lunar current systems averaged over the winters with and without a stratospheric sudden warming event. The current intensity is globally enhanced during stratospheric sudden warming winters. Figures $47 \mathrm{c}$ and $47 \mathrm{~d}$ show the corresponding results for Sq current systems. It is noted that the contour intervals are different between the results for $L(a, b)$ and $\mathrm{Sq}(\mathrm{c}, \mathrm{d})$. During a stratospheric sudden warming, the northern Sq current focus moves to earlier local times while the southern $\mathrm{Sq}$ focus moves to later local times. Yamazaki 


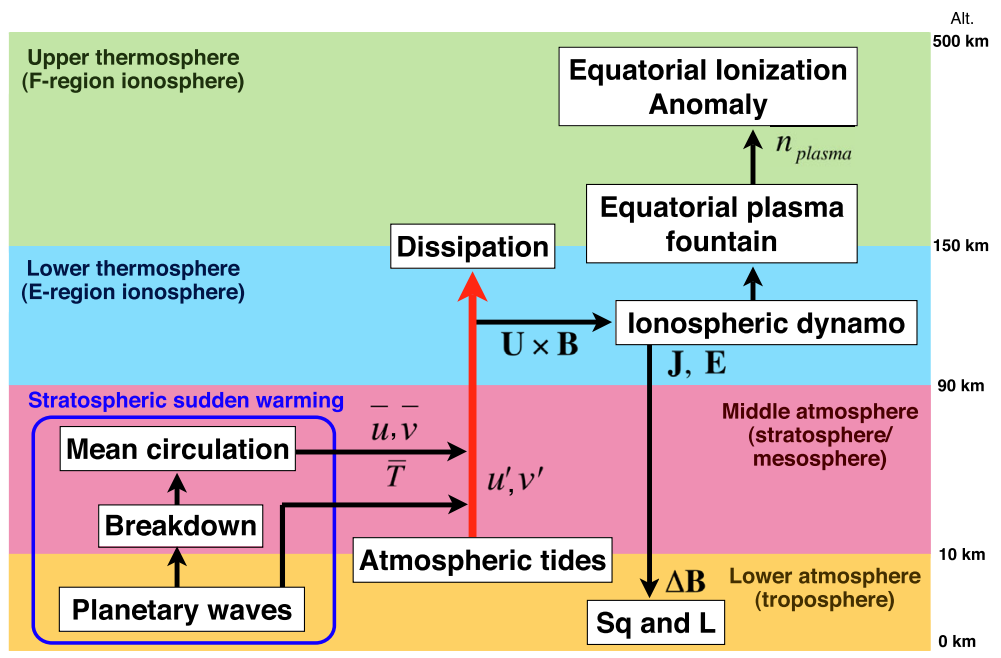

Fig. 48 A schematic diagram for vertical atmospheric coupling during stratospheric sudden warmings. Different colors indicate different regions of the atmosphere; namely, the lower atmosphere $(0-10 \mathrm{~km})$, middle atmosphere $(10-90 \mathrm{~km})$, lower thermosphere $(90-150 \mathrm{~km})$, and upper thermosphere $(150-500 \mathrm{~km})($ Color figure online)

et al. (2012c) reported similar changes in the Sq current pattern during January-2006 and January-2009 stratospheric sudden warming events. They suggested that an enhancement in the solar anti-symmetric " $(2,3)$ mode" semidiurnal tide can cause the observed changes in the Sq current pattern. Geomagnetic daily variations during stratospheric sudden warming events are affected by changes in both solar and lunar currents. These two contributions cannot be separated for individual events. The separation becomes possible if a number of stratospheric sudden warming events are analyzed all together. Based on such a statistical approach, Yamazaki (2014) showed that at the magnetic equator, semidiurnal geomagnetic variations due to solar and lunar currents are in phase near new moon and full moon, and out of phase during half moon, which gives rise to the recurrent lunar dependent onset in the afternoon counter electrojet during stratospheric sudden warmings (see Fig. 45).

Stratospheric sudden warming effects on the ionospheric dynamo have a broad impact on the ionosphere. As described in Sect. 3.11, changes in the equatorial electrojet strength is closely related to changes in the F-region electric field and plasma density. During stratospheric sudden warming events, dynamo-region electric fields are disturbed due to enhanced solar and lunar tidal forcing. The modulation of the equatorial plasma fountain has been observed in measurements of the F-region equatorial $\mathbf{E} \times \mathbf{B}$ drift (e.g., Chau et al. 2009; Fejer et al. 2011). Also, the impact on the equatorial anomaly has been revealed by ground-based and satellite measurements of the F-region plasma density (e.g., Goncharenko et al. 2010a, 2010b; Yue et al. 2010; Pancheva and Mukhtarov 2011; Lin et al. 2012). Figure 48 schematically illustrates the vertical coupling of the atmosphere during stratospheric sudden warmings. Understanding of those physical processes has been facilitated by numerical models of the "whole atmosphere" covering the height range from the surface to the exobase (e.g., Fuller-Rowell et al. 2010; Jin et al. 2012; Pedatella et al. 2014b). 


\subsubsection{Storm Effect}

During storm times, a large amount of electromagnetic energy is deposited into the highlatitude ionosphere. The resulting disturbances extend into lower latitudes, and have a significant influence on the ionospheric electrodynamics at middle and low latitudes. In this section, we discuss how these storm-time processes affect the ionospheric dynamo currents that are responsible for the $\mathrm{Sq}$ variations during quiet times.

Before going into the main subject, it is important to remember that there are two types of classification for Sq (Sect. 1.2). One is "descriptive" (or "statistical") classification, which focuses on how to derive Sq. The other is "physical" (or "causal") classification, which puts an emphasis on what Sq represents. In the first approach, $\mathrm{Sq}$ is often defined as the geomagnetic daily variation averaged over the five quietest days of a month. Thus, the geomagnetic daily variations during storm periods are usually not considered as Sq. On the other hand, in the physical classification, which we use in this review, Sq can be defined even during a storm as long as it represents the magnetic effect of ionospheric dynamo currents. However, since the geomagnetic daily variation during storm periods is dominated by the effect of magnetospheric currents, a careful analysis of the data is required to isolate the effect of ionospheric dynamo currents.

Evidence suggests that even under active conditions, ionospheric dynamo processes continue, and thus the Sq current system exists. For example, Hibberd's technique (Sect. 3.8) makes it possible to separate the geomagnetic daily variation associated with ionospheric currents even during a storm. The technique involves $H$ data from two stations located in the same longitude; one is on the equator side of the $\mathrm{Sq}$ focus and the other on the polar side. The drawback of this technique is that it removes a part of ionospheric contributions, and it is not possible to know how much ionospheric effects are removed.

Another convenient method to remove magnetospheric contributions from the data is to take the difference in $H$ between an equatorial station and off-equatorial station in the same longitude (Sect. 2.5). Although this technique also removes a part of ionospheric contributions, the influence is less significant compared to Hibberd's technique. This is because ionospheric currents are much enhanced at the magnetic equator owing to the Cowling effect (Sect. 2.3). Therefore, the difference $\Delta H$ is dominated by the contribution of ionospheric currents at the location of the equatorial station, i.e., the equatorial electrojet.

Rastogi (1977), analyzing the difference in $H$ at Huancayo $\left(12.0^{\circ} \mathrm{S}, 75.3^{\circ} \mathrm{W}\right)$ and Fuquene $\left(5.4^{\circ}, 73.7^{\circ}\right)$, recognized the occurrence of the counter electrojet during geomagnetic storms. Later, Kikuchi et al. $(2003,2008)$ demonstrated how the penetration of high-latitude electric fields to lower latitudes can cause an enhancement and reduction (or reversal) in the equatorial electrojet. High-latitude electric fields penetrate to lower latitudes, most evidently when the magnetospheric convection suddenly increases or decreases. Under steady-state conditions, the inner magnetosphere is effectively shielded from the magnetospheric convection field (Wolf 1995). In other words, the middle- and lowlatitude ionosphere is shielded from the effect of high-latitude electric fields. However, when the magnetospheric convection abruptly changes, the middle- and low-latitude ionosphere is temporarily exposed to the influence of high-latitude electric fields until the magnetospheric configuration readjusts and a new state of shielding is established. The time scale for the shielding processes is typically less than one hour (Kikuchi et al. 2000; Peymirat et al. 2000) but subject to magnetospheric conditions (Senior and Blanc 1984; Maruyama et al. 2007). During the main phase of a geomagnetic storm, the penetration electric field is sometimes observed to last for several hours without decay (Kelley et al. 2003; Huang et al. 2005a, 2005b). 
In addition to the penetration electric fields from high altitudes, the storm-time ionospheric currents are under the influence of the disturbance dynamo (Blanc and Richmond 1980; Fuller-Rowell et al. 2002). The disturbance dynamo refers to the ionospheric dynamo processes due to disturbance winds generated by enhanced energy and momentum deposition into the high latitude ionosphere during storm periods. Enhanced Joule heating $\mathbf{J} \cdot(\mathbf{E}+\mathbf{U} \times \mathbf{B})$ and Ampère force $\mathbf{J} \times \mathbf{B}$ effectively drive disturbance winds in the highlatitude thermosphere, which spread globally and produce electric fields and currents at lower latitudes. Blanc and Richmond (1980) were the first to describe the electrodynamic response to disturbance winds using a numerical model. At middle and low latitudes, westward winds are established owing to Coriolis force acting on the equatorward disturbance winds from higher latitudes. These westward winds cause a build up of positive and negative charges at the dusk and dawn terminators, respectively. The resulting polarization electric fields are westward on the dayside and eastward on the nightside, which oppose to the normal quiet-time fields. Therefore, the disturbance dynamo acts to produce an "anti-Sq" current system on the dayside ionosphere. The westward disturbance winds, and thus the electrodynamic disturbances, can persist for many hours after the high-latitude energy input ceases (Huang et al. 2005a, 2005b). Accordingly, the disturbance dynamo effects are often detected during the recovery phase of a storm (e.g., Le Huy and Amory-Mazaudier 2005; Zaka et al. 2009; Yamazaki and Kosch 2015).

Observations of equatorial electric fields and currents have shown that both the penetration of high-latitude electric fields and the disturbance dynamo play a significant role for storm-time disturbances (Fejer and Scherliess 1995; Yamazaki and Kosch 2015). Figure 49 shows the equivalent current systems associated with (a) Sq, (b) disturbance dynamo, and (c) penetration electric field, derived from the NCAR TIE-GCM. The results were obtained from three model runs. The first simulation was performed for solar minimum equinox conditions, without including any external high-latitude forcing, which we call "Q (quiet) run". The second simulation is the same as the $\mathrm{Q}$ run except that the high-latitude convection pattern and auroral particle precipitation were specified using empirical models by Heelis et al. (1982) and Roble and Ridley (1987), respectively, for $K p=5^{-}$conditions. We call it "D (disturbance) run". In the third run, the high-latitude electric field and auroral precipitation were turned off, but the disturbance winds from the D run were used to drive ionospheric dynamo. We call it "M (mixed) run". All those simulations were run until a diurnally reproducible state is achieved. The Sq current system can be immediately obtained from the Q run, which is shown in Fig. 49a. The simulated Sq current system shows the expected current pattern with a counterclockwise dayside vortex in the Northern Hemisphere, and a clockwise vortex in the Southern Hemisphere. The ionospheric current system associated with the disturbance dynamo (Fig. 49b) is obtained as the difference between the M run and Q run. The anti-Sq current pattern is visible on the dayside ionosphere. Observational evidence for the anti-Sq current system is limited to the study by Le Huy and Amory-Mazaudier (2008), where they observed a notably reduced Sq current system during the recovery phase of the storm on 25 November 2001. The effect of the penetration electric field can be derived as the difference between the D run and M run. The results (Fig. 49c) reveal strong DP2 currents at high latitudes (above $60^{\circ}$ magnetic latitudes). These currents leak into lower latitudes on both dayside and nightside. It would be difficult to reproduce the current system shown in Fig. 49c using observations because magnetometer data during disturbed periods contain the effect of both magnetospheric and ionospheric currents. 

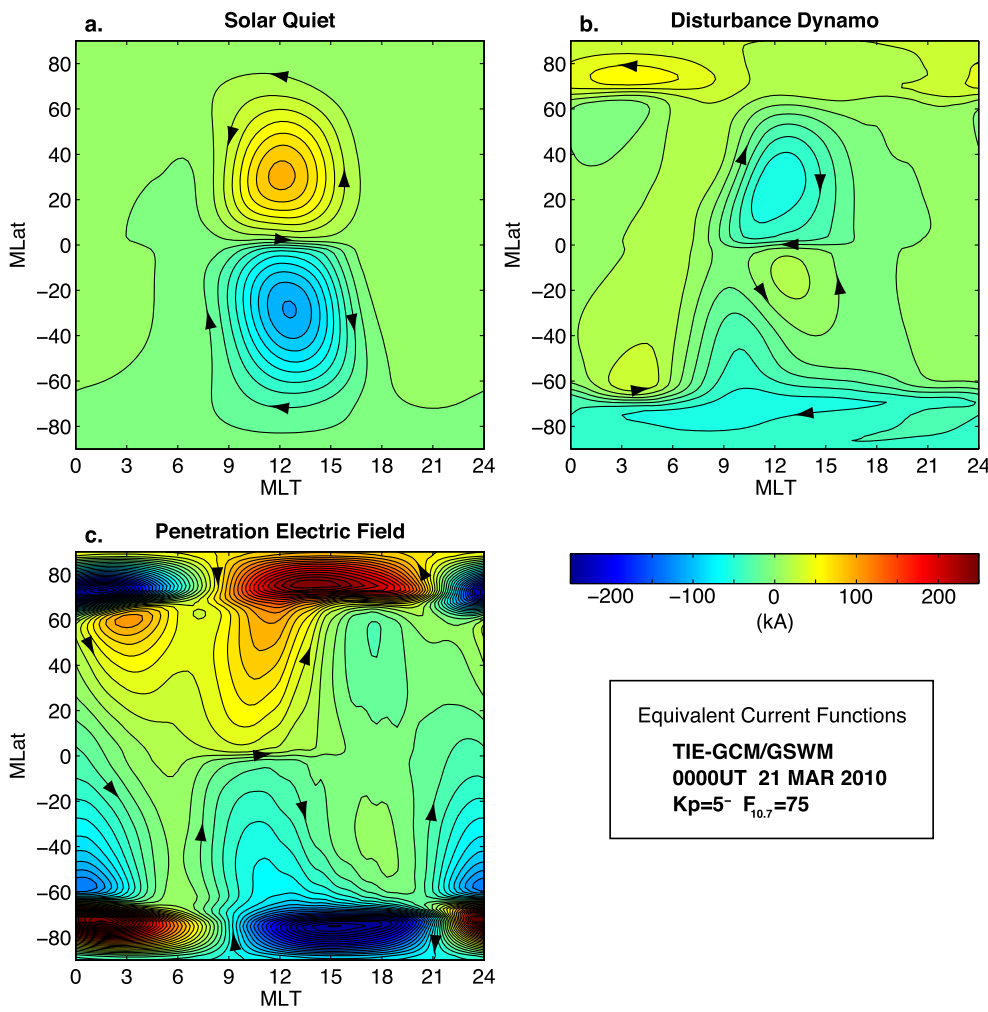

Fig. 49 Equivalent ionospheric current systems simulated by the NCAR TIE-GCM under active geomagnetic conditions $\left(K p=5^{-}\right)$. (a) The background Sq current system. (b) The current system due to the disturbance dynamo effect. (c) The current system due to the penetration electric field

\subsection{Solar Flare and Eclipse Effects}

Solar flare is a transient radiation burst that occasionally comes from the sun following an eruption in the solar chromosphere. Solar flares sometimes cause short-lived $(\sim 1 \mathrm{hr})$ geomagnetic disturbances, known as solar flare effects (SFE) or geomagnetic crochets. An interrelation between the optical solar flare and the geomagnetic crochet was first identified by Carrington and Hodgson during the extreme event on 1 September, 1859 (Cliver and Dietrich 2013). Examples of geomagnetic crochets are presented in Fig. 50 (after Cliver and Svalgaard 2004), along with soft X-ray flux (1-8 ^) by the GOES-12 satellite. McNish (1937a, 1937b) showed that equivalent current vectors for geomagnetic crochets are similar in direction with the equivalent current vectors for Sq. The implication is that geomagnetic crochets are due to an augmentation of the Sq current system. An increase in the solar EUV and X-ray fluxes during solar flares causes an enhancement of ionospheric conductivities and thus ionospheric Sq currents. Nagata (1966) reviewed the development of understanding of geomagnetic crochets.

Later studies recognized the difference in the position of the current foci between equivalent current systems of Sq and geomagnetic crochet (e.g., Volland and Taubenheim 1958; Veldkamp and Van Sabben 1960; Yasuhara and Maeda 1961; van Sabben 1961, 1968; Curto et al. 1994; Gaya-Piqué et al. 2008). The differences were generally attributed to the differ- 

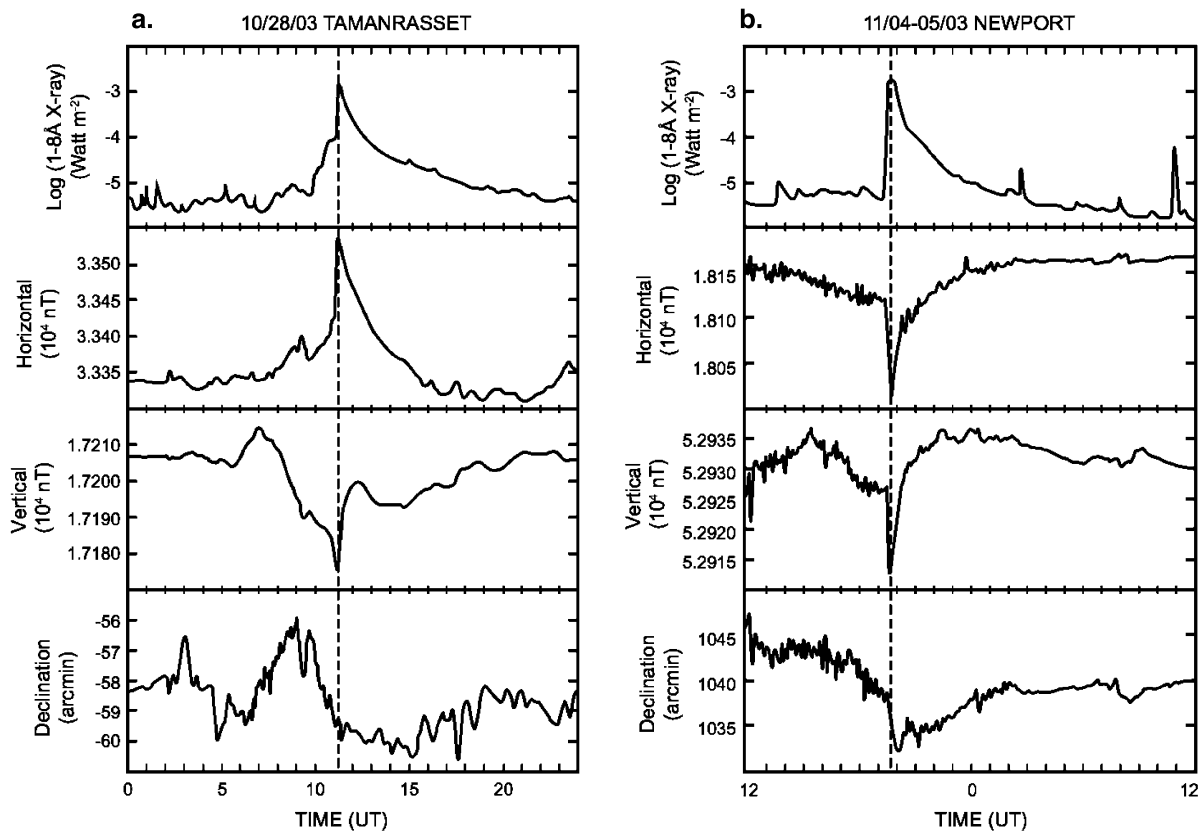

Fig. 50 Geomagnetic crochets (or solar flare effects) recorded (a) at Tamanrassert $\left(22.8^{\circ} \mathrm{N}, 5^{\circ} \mathrm{E}\right)$ on 28 October 2003, and (b) at Newport $\left(48.3^{\circ} \mathrm{N}, 117.1^{\circ} \mathrm{W}\right)$ on 4 November 2003. In each case, the top panel shows the soft X-ray flux (1-8 $\mathrm{A})$ measured by the GOES-12 satellite, and the bottom three panels show geomagnetic data. From Cliver and Svalgaard (2004)

ent heights where the two types of ionospheric currents flow. That is, Sq currents are mostly restricted to the E region height, while ionospheric currents during solar flares extend down to the $\mathrm{D}$ region due to enhanced conductivities.

A geomagnetic crochet often consists of two components (Richmond and Venkateswaran 1971); a "fast component" is presumably produced by a rapid increase in the EUV radiation and a "slow component", which peaks a few minutes after the fast component, corresponds to a slow increase in soft $\mathrm{X}$ rays. Although the conductivity enhancement during solar flares is limited to the dayside ionosphere, several authors found evidence of solar flare effects during the nighttime (Ohshio et al. 1963; Sastri 1975; Sastri and Murthy 1975).

Nagata (1952) reported that the amplitude of geomagnetic crochets in $H$ observed at Huancayo $\left(12.0^{\circ} \mathrm{S}, 75.3^{\circ}\right)$ is much greater than those at mid-latitude stations Kakioka $\left(36.2^{\circ} \mathrm{N}, 140.2^{\circ} \mathrm{E}\right)$ and Watheroo $\left(30.3^{\circ} \mathrm{S}, 115.9^{\circ} \mathrm{E}\right)$. Forbush and Casaverde (1961) showed that the amplitude of $H$-component geomagnetic crochets at Peruvian stations is enhanced near the magnetic equator in a similar manner to the Sq amplitude. Rastogi et al. (1999) demonstrated that geomagnetic crochets in $H$ near the magnetic equator exhibit a positive perturbation during the normal (eastward) equatorial electrojet and a negative perturbation during the counter electrojet. Rastogi (2003), in a survey of geomagnetic crochets in $H$ at Huancayo, found a peculiar event on 3 January 1960, where a negative perturbation was recorded while the mean $H$ field was well above the night time level. Yamazaki et al. (2009) examined the global morphology of geomagnetic crochets for such unusual events. Figure 51 (after Yamazaki et al. 2009) shows geomagnetic crochets in the magneticnorthward $N$ component observed at equatorial stations during the solar flare events on (a) 
a.

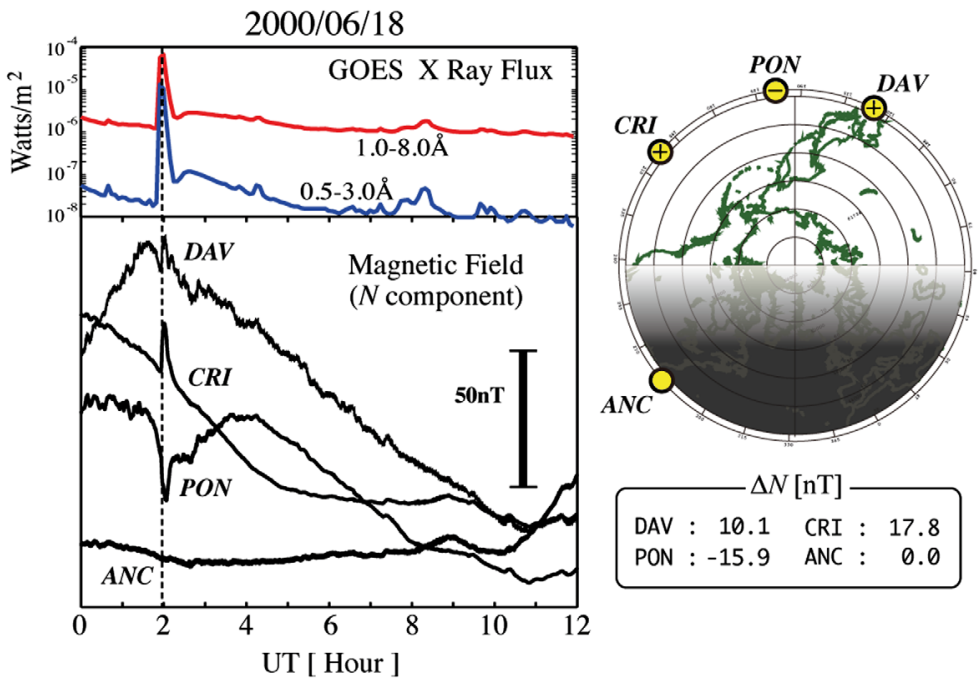

b.
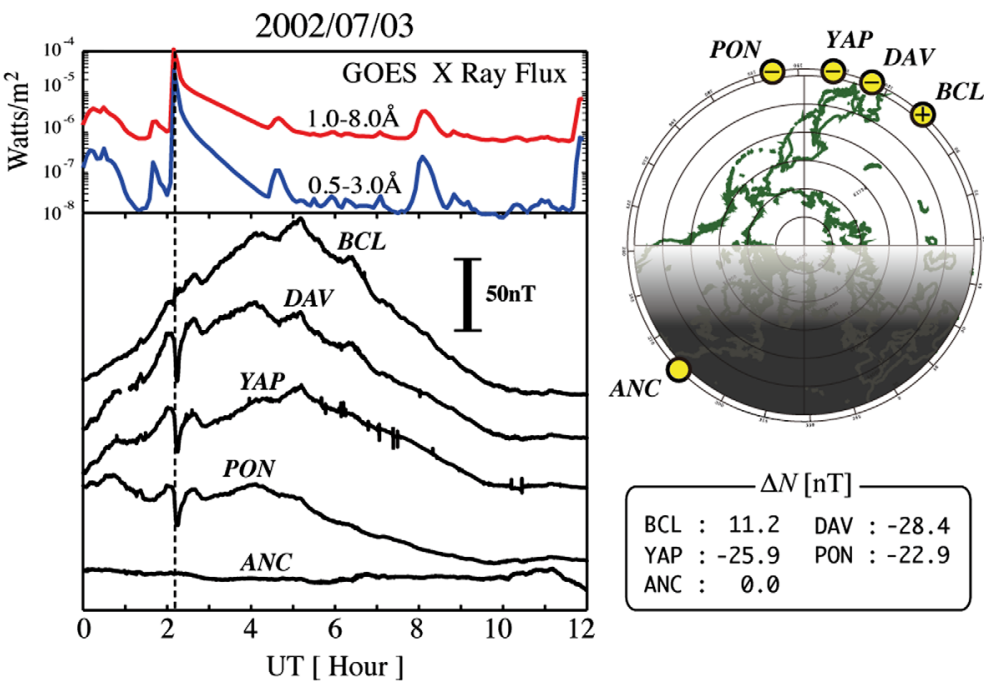

Fig. 51 Examples of solar flare events that are accompanied by a reduction in the $H$ field near the magnetic equator. From Yamazaki et al. (2009)

18 June, 2000 and (b) 3 July, 2002. The mechanism for those counter-Sq crochets at the magnetic equator is not understood.

In contrast to solar flares that give rise to an increase in the ionospheric conductivity, solar eclipses cause a temporary reduction in the ionospheric conductivity (by $\sim 50 \%$ ) along the path of the Moon's umbra. Early models showed how ionospheric currents would be modulated around the eclipse shadow, which in turn causes magnetic variations on the ground (Chapman 1933; Nagata et al. 1955). The first successful observation of the geomagnetic effect was made by Kato (1960) during the solar eclipse of 12 October, 1958. 


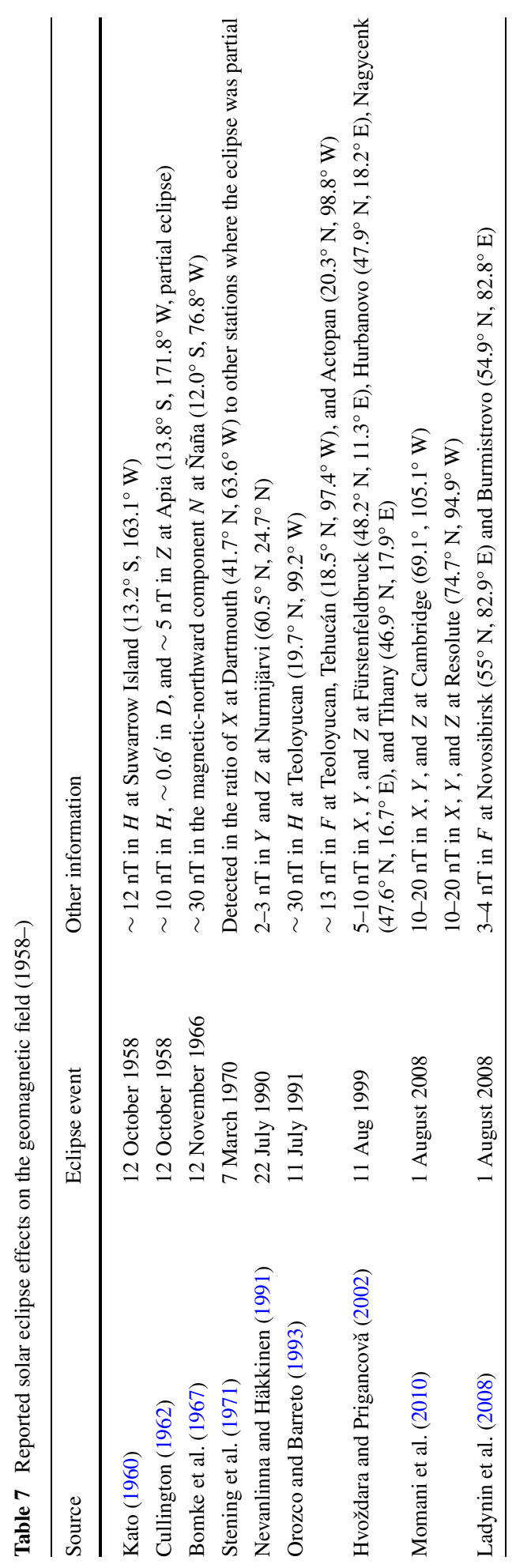


A reduction by $\sim 12 \mathrm{nT}$ was detected in the horizontal component geomagnetic field at a low-latitude station Suwarrow Island $\left(13.2^{\circ} \mathrm{S}, 163.1^{\circ} \mathrm{W}\right)$. The peak reduction was observed approximately $10 \mathrm{~min}$ after the maximum phase of the eclipse. Earlier attempts to observe geomagnetic solar eclipse effects can be found in review articles by Matsushita (1966) and Rastogi (1982). Following Kato (1960), other studies have also confirmed the reality of the solar eclipse effect on the geomagnetic field (see Table 7). The typical duration of the solar eclipse effect is $\sim 1 \mathrm{hr}$, similar to the solar flare effect. The solar eclipse effect tends to reduce the background Sq field, while the solar flare effect tends to enhance it. The magnitude of the solar eclipse effect decreases with the distance from the Moon's shadow, reducing to the level of noise at a distance of $\sim 500 \mathrm{~km}$. Takeda and Araki (1984b), using a dynamo model, suggested that the solar eclipse effect may be observed in the opposite hemisphere through electrodynamic coupling between the Northern Hemisphere and the Southern Hemisphere. There is so far no observational evidence to support this prediction. Aside from the short-lived effect, some studies noted a high occurrence rate of the counter electrojet on the day of eclipse (Tomás et al. 2008; Choudhary et al. 2011). This seems to be related to the fact that a solar eclipse, by definition, occurs at new moon, which favors the occurrence of the counter electrojet (e.g., Rastogi 1974). Further studies are required to identify the mechanism.

\subsection{Long-Term Variation}

Ionospheric currents undergo changes on time scales longer than a solar cycle ( $\sim 11$ years). The long-term variations arise from (1) long-term changes in solar activity, (2) secular variation of the geomagnetic field, and (3) long-term changes in the upper atmosphere. Longterm changes exist in solar activity, as evident from the fact that the maximum sunspot number varies from one solar maximum to the next. It can cause long-term changes in the ionospheric conductivity. The secular variation of the geomagnetic field $\mathbf{B}$ affects the ionospheric dynamo by altering the $\mathbf{U} \times \mathbf{B}$ field as well as ionospheric conductivities (see (4)(8)). Takeda (1996) examined this effect using a dynamo model. He found that a reduction in the magnetic dipole moment causes an increase in the Sq current intensity because the effect of enhanced ionospheric conductivities overcomes the effect due to the reduction in the U $\times$ B field. Cnossen and Richmond (2013), using the Coupled Magnetosphere-IonosphereThermosphere (CMIT) model (Wiltberger et al. 2004), compared ground-level Sq variations calculated with the geomagnetic field of 1908 and 2008. Figure 52 (after Cnossen and Richmond 2013) shows the difference in the background geomagnetic field between 2008 and $1908(\mathrm{a}-\mathrm{c})$ as well as the difference in the Sq amplitude caused by the secular change in the geomagnetic field $(\mathrm{d}-\mathrm{f})$. The largest changes in the Sq amplitude occur near the magnetic equator in the South American region (on the order of several $10^{-4} \mathrm{nT}$ per day) because of the displacement in the equatorial electrojet axis. Finally, long-term changes in the upper atmosphere is another possible cause for the long-term variations of the ionospheric dynamo. Increasing concentrations of $\mathrm{CO}_{2}$ in the lower atmosphere are expected to cool the middle and upper atmosphere, where $\mathrm{CO}_{2}$ acts as an effective radiative coolant. This leads to further changes in the densities and compositions in the thermosphere and ionosphere (Cnossen 2012; Laštovička et al. 2012), which can affect the ionospheric conductivities. Besides, tidal forcing from the lower atmosphere may change over the course of time. Decreasing trends have been generally observed in the diurnal and semidiurnal tidal amplitudes in the middle atmosphere (Ross and Walterscheid 1991; Bremer et al. 1997; Jacobi and Kürschner 2006).

Studies have shown that the solar effect is the dominant cause of the long-term change in the Sq variation on the time scale of decades. In Fig. 53 (after Le Mouël et al. 2005), time 
a.

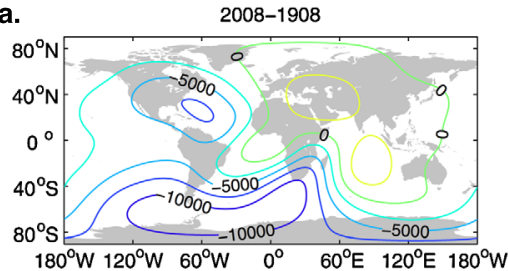

c.

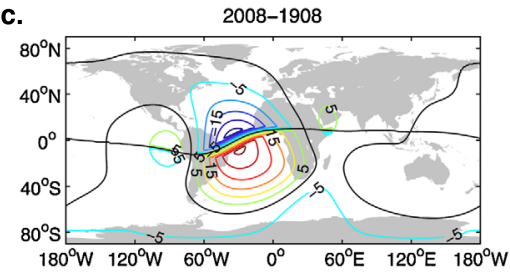

b. $-1908-2008$

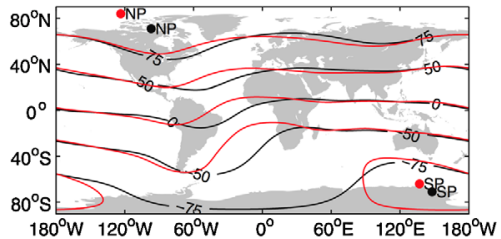

d. $\quad \triangle B$ amplitude North $\quad \mathrm{mf} 2008-\mathrm{mf} 1908$

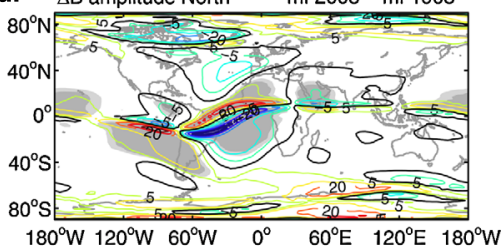

e.

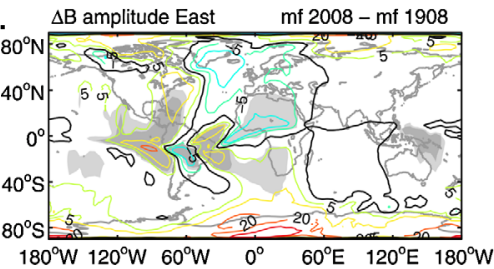

Fig. 52 The CMIT simulation results from Cnossen and Richmond (2013). (a) The difference in the geomagnetic field strength between 1908 and 2008. (b) The inclination of the magnetic field in 1908 and 2008. (c) The difference in the inclination between 1908 and 2008 in degrees. (d) The difference in the daily Sq ranges in the magnetic-northward $(N)$ component between 1908 and 2008. (e) The same as (d) but for the magnetic-eastward $(E)$ component. (f) the same as (d) but for the $Z$ component. Panels (a), (d-f) are in nanoteslas, while panels (b) and (c) are in degrees. Light and dark shading in panels (d-f) indicate $95 \%$ and $99 \%$ statistical significance, respectively

series are depicted for the daily ranges in the $X-, Y$-, and $Z$-component geomagnetic field at Eskdalemuir $\left(55.3^{\circ} \mathrm{N}, 3.2^{\circ} \mathrm{W}\right)$, as well as those for the geomagnetic activity index $a a$ and sunspot number. The daily ranges closely follow the variations in the $a a$ index and sunspot number, not only in the yearly average (dots and thin lines) but also in the 11-year running average (thick lines). Figure 53 includes all the data regardless of geomagnetic activity, but the pattern of the long-term variation is largely the same when the analysis is limited to geomagnetically quiet days only (Le Mouël et al. 2005; Macmillan and Droujinina 2007; Torta et al. 2009). It is, thus, possible that both solar radiation activity and solar-wind electric field play a role for the long-term variations of Sq. The long-term variations in solar activity (both $a a$ and sunspot number) show a monotonic increase from 1920 to 1960, a decrease to $\sim 1972$, again an increase until 1992, and a decrease to 2000. The cause of the long-term variation in solar activity is not understood.

Other researchers attempted to "remove" the effect of solar activity from Sq data in order to determine the long-term variations other than those caused by solar activity. Sellek (1980) and Schlapp et al. (1990) examined the residual Sq amplitude after subtracting the linear fit of the sunspot number to the Sq amplitude. They found significant trends (on the order of $10^{-4} \mathrm{nT}$ per day) in the residual data at some stations, but the trends were not always consistent among the stations. Elias et al. (2010), using a similar technique, showed significant trends in the residual Sq amplitude at Apia $\left(13.8^{\circ} \mathrm{N}, 171.8^{\circ} \mathrm{W}\right)$, Fredericksburg $\left(38.2^{\circ} \mathrm{N}\right.$, 
Fig. 53 Year-to-year variations of the daily range in $X, Y$, and $Z$ at Eskdalemuir $\left(55.3^{\circ} \mathrm{N}, 3.2^{\circ} \mathrm{W}\right)$ during 1911-1999, and year-to-year variations of the $a a$ index and sunspot number. The thick lines represent 11-yr running mean values. From Le Mouël et al. (2005)
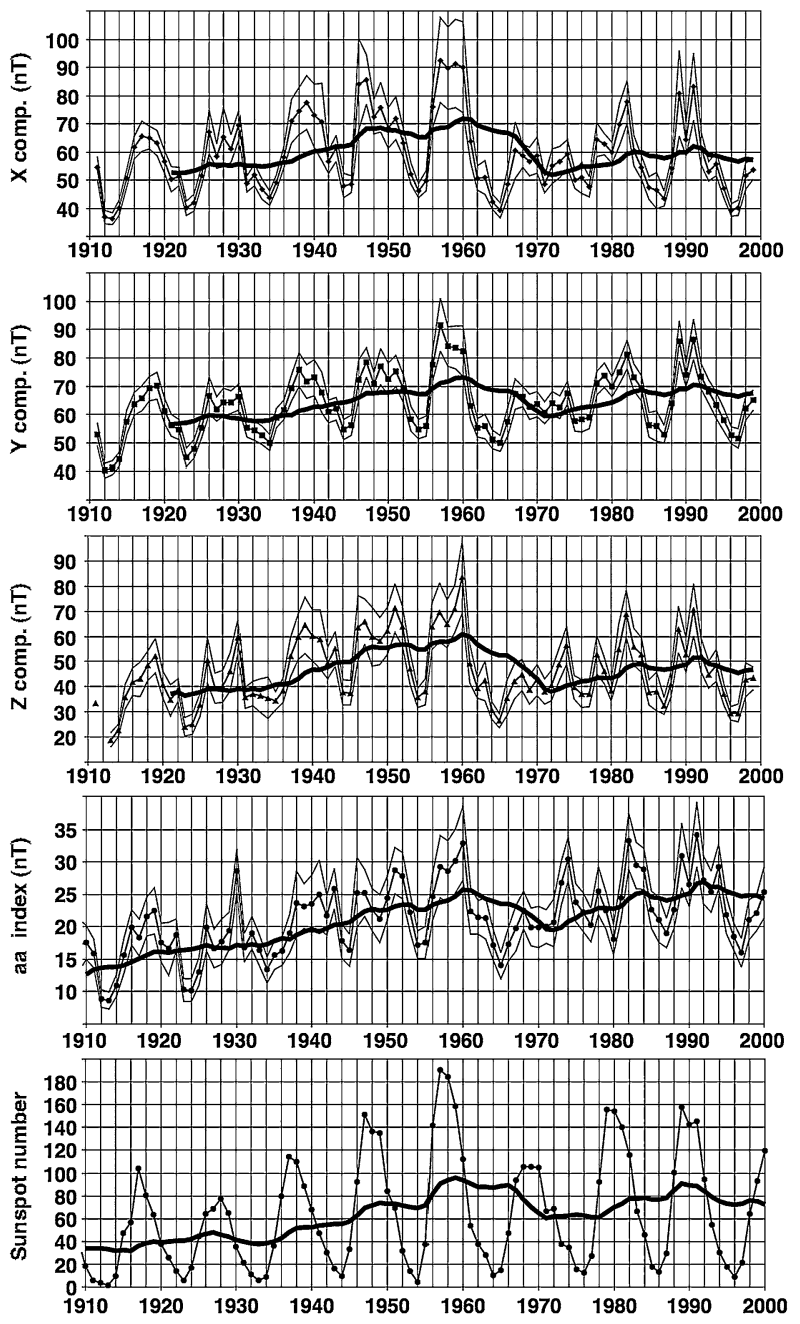

$\left.77.3^{\circ} \mathrm{W}\right)$, and Hermanus $\left(34.4^{\circ} \mathrm{S}, 19.2^{\circ} \mathrm{E}\right)$ during 1960-2001. The trends in the residual Sq amplitudes were found to be all positive: $2.2 \times 10^{-4} \mathrm{nT} /$ day, $1.3 \times 10^{-4} \mathrm{nT} /$ day, and $2.1 \times 10^{-4} \mathrm{nT} /$ day, respectively. Elias et al. (2010) showed that these positive trends can be explained in part as the effect of long-term changes in the ionospheric conductivities. However, the observed long-term trends in the residual $\mathrm{Sq}$ amplitudes were greater than those expected from the conductivity changes; thus, other contributions such as $\mathrm{CO}_{2}$ might also play a role. de Haro Barbas et al. (2013) compared these trends with the results obtained from the CMIT model, confirming the role of the secular variation of the geomagnetic field in the long-term changes in Sq. In contrast to these results, Jarvis (2005) found a longterm decrease in the diurnal and semidiurnal spectral power at Lerwick $\left(61.1^{\circ} \mathrm{N}, 358.8^{\circ} \mathrm{E}\right)$, Niemegk $\left(52.1^{\circ} \mathrm{N}, 12.7^{\circ} \mathrm{E}\right)$, and Tucson $\left(32.2^{\circ} \mathrm{N}, 249.3^{\circ} \mathrm{E}\right)$ after removal of solar activity and geomagnetic activity effects. Shinbori et al. (2014) also found negative trends in the residual Sq amplitude ( $\sim 10^{-4} \mathrm{nT}$ per day) at many stations worldwide. Both Jarvis (2005) and Shinbori et al. (2014) used the $H$-component geomagnetic field, the same as Elias et al. 
(2010) and de Haro Barbas et al. (2013). The discrepancies in the long-term trend reported by different authors are probably due to different approaches used in parameterizing solar activity effects.

\subsection{Longitudinal Dependence}

\subsubsection{Sq}

The strength and shape of the equivalent Sq current system vary with longitude (e.g., Matsushita 1967; Campbell 1989a). The longitudinal variation of the main field B modulates the $\mathbf{U} \times \mathbf{B}$ field and ionospheric conductivities (see (4)-(8)), causing the longitudinal variation in the dynamo-region currents as well as inter-hemispheric field-aligned currents (Stening 1971; Le Sager and Huang 2002b). The longitudinal variation of the Sq current intensity was recently presented by Pedatella et al. (2011) based on satellite magnetometer data and by Stening and Winch (2013) based on ground magnetometer data. Figure 54 compares the two results, revealing significant differences in the pattern of the longitudinal variation. Some discrepancies are expected from the different data sets and techniques used. Pedatella et al. (2011) used the CHAMP vector magnetometer observations during 2006-2008, while Stening and Winch (2013) used magnetic data from 130 ground stations during 1964-1965, originally analyzed by Winch (1981). For the baseline of Sq, Pedatella et al. (2011) used the POMME6.1 geomagnetic field model (Maus et al. 2010), while Stening and Winch (2013) used the daily mean value. Moreover, Fig. 54a (from Pedatella et al. 2011) is based on the Sq current function at 1200 LT, while Fig. 54b (from Stening and Winch 2013) is based on the positive and negative peaks of the Sq current function at different universal times (e.g., 0000 UT corresponds to $180^{\circ}$ longitude). The reconciliation of the two results seems to be difficult even if all these differences are taken into consideration. TIE-GCM simulation results are presented in Fig. 54c in a similar format as Fig. 54a. Interestingly, some features are in agreement with satellite measurements by Pedatella et al. (2011) and others features are in agreement with ground measurements by Stening and Winch (2013). For the Northern Hemisphere, the model predicts strong Sq currents over the American sector throughout the year, which is consistent with the ground measurements. (Note, however, that the peak in the model is shifted to the east by $20-30^{\circ}$ compared to the observation.) Meanwhile, for the Southern Hemisphere, the model shows a longitudinal variation similar to the satellite results. More work will be necessary to achieve a consensus among ground, satellite, and model results.

Since the ionospheric dynamo is strongly organized in magnetic coordinates, the Sq focus tends to appear at the same magnetic latitude at different longitudes. In other words, the geographic latitude of the Sq focus changes with longitude. Figure 55a (from Çelik 2013) traces the latitudinal position of the northern and southern Sq current foci. It can be seen that the Sq current foci move along the magnetic equator. (See, for example, Fig. 8 for the longitudinal pattern of the magnetic equator.) TIE-GCM results are presented in Fig. 55b. The results are consistent with the ground-based observations shown in Fig. 55a. Similar results can also be obtained from the comprehensive model by Sabaka et al. (2002, 2015; see also Matzka et al. 2009).

\subsubsection{EEJ}

Features of the equatorial electrojet have been studied at different longitudes: e.g., for the Pacific region $\sim 160^{\circ} \mathrm{W}$ by Mason (1963); for the South American region $\sim 75^{\circ} \mathrm{W}$ by 


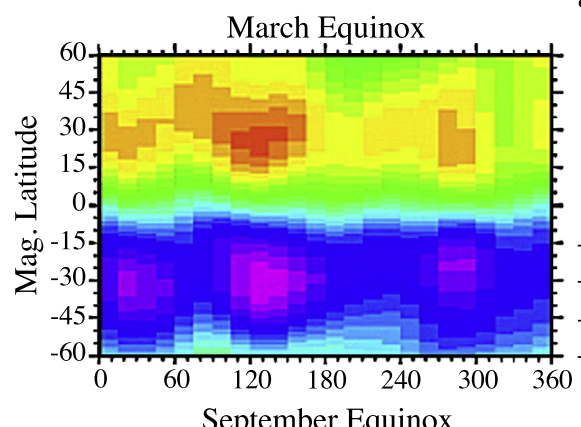

a.

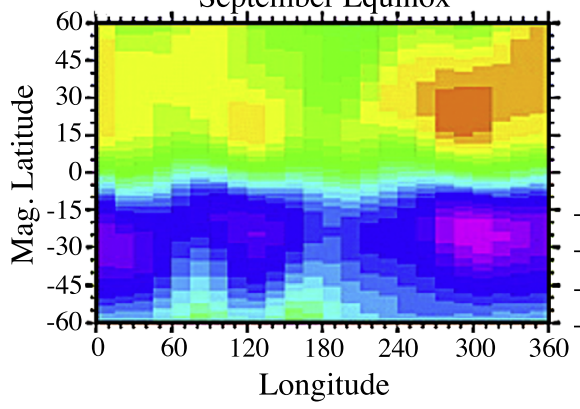

June Solstice

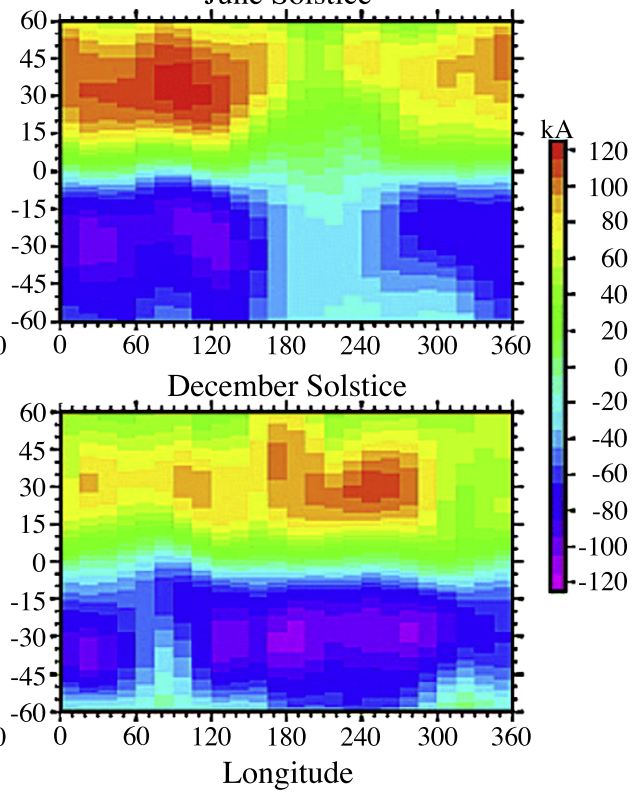

b.
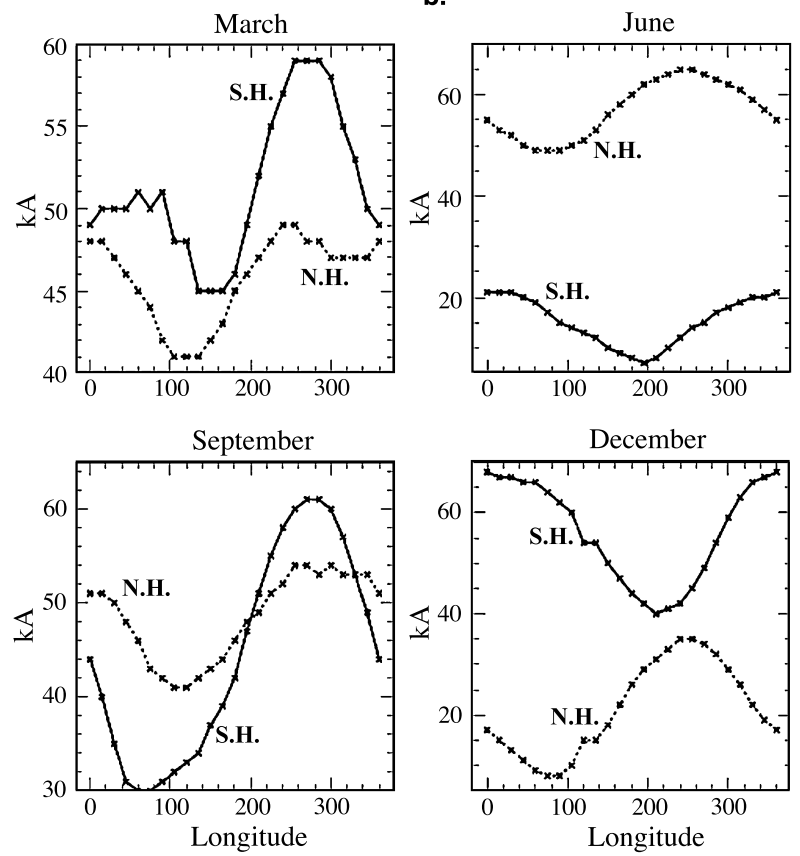

Fig. 54 The longitudinal dependence of the Sq current intensity, derived from (a) CHAMP satellite data during 2006-2008 by Pedatella et al. (2011); (b) ground-based magnetometer measurements during 1964-1965 by Stening and Winch (2013); and (c) TIE-GCM with $F_{10.7}=120 \mathrm{sfu}$. In panel (b), the solid and dashed lines correspond to the results for the Southern Hemisphere and the Northern Hemisphere, respectively 


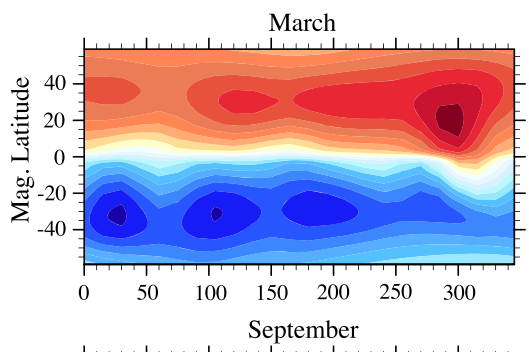

c.
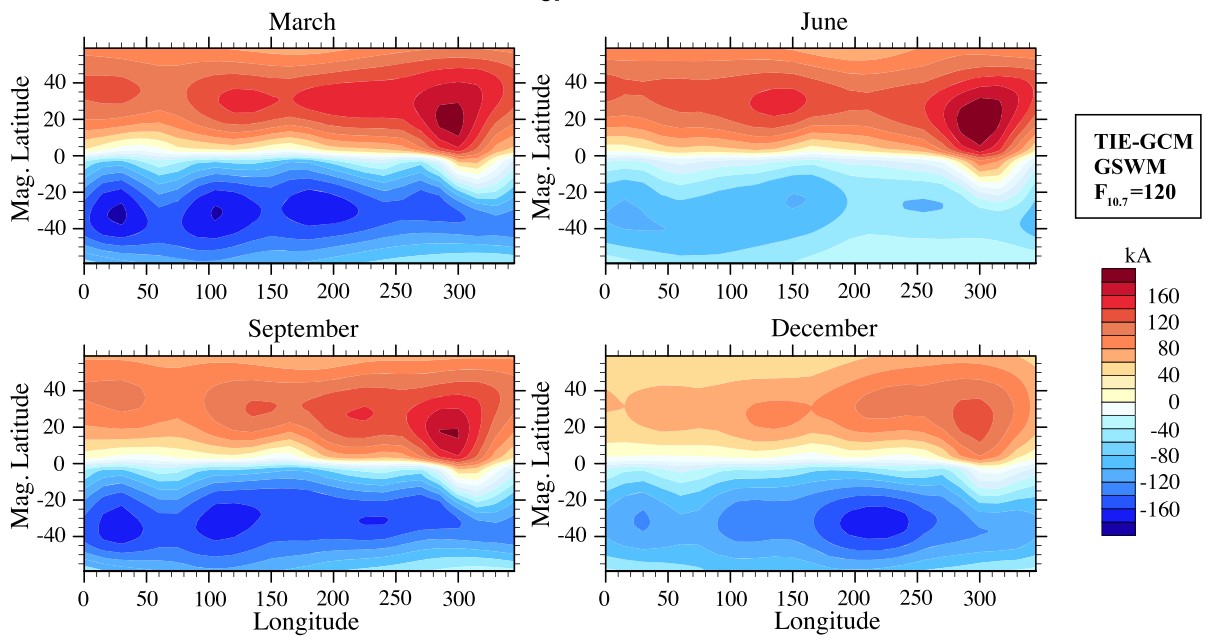

Fig. 54 (Continued)

Forbush and Casaverde (1961), Hutton (1967); 62 ${ }^{\circ} \mathrm{W}$ by Rastogi et al. (2008); $\sim 45^{\circ} \mathrm{W}$ by Rigoti et al. (1999), Rastogi et al. (2010); for the African region $\sim 5^{\circ} \mathrm{E}$ by Onwumechili (1959), Ogbuehi and Onwumechilli (1963); 11 ${ }^{\circ}$ E by Doumouya et al. (1998); $\sim 16^{\circ} \mathrm{E}$ by Fambitakoye and Mayaud (1976a, 1976b); $\sim 39^{\circ}$ E by Rastogi and Chandra (2015); for the Central Asian region $\sim 77^{\circ}$ E by Rabiu and Nagarajan (2007), Rabiu et al. (2013); $\sim 81^{\circ}$ E by Rastogi et al. (2004); for the East Asian region $\sim 125^{\circ}$ E by Yamazaki et al. (2010). Comparisons of the equatorial electrojet intensities at various longitude sectors have revealed a strong dependence on the main field B (Rastogi 1962; Doumouya et al. 2003; Abdul Hamid et al. 2015). Figure 56a (from Doumouya et al. 2003) illustrates the longitudinal dependence of the noon-time equatorial electrojet during the International Equatorial Electrojet Year (IEEY) (Abdu 1992), September 1991-March 1993. The equatorial electrojet is strongest over the South American sector where the main field is weakest. These observations have been interpreted as arising from the ionospheric conductivity dependence on the main field. Rastogi (2006), however, pointed out that the equatorial electrojet in the Vietnamese sector is stronger than in the Indian sector, which is against the expectation from the distribution of the main field. The results by Rastogi (2006) suggest that there should be other mechanisms that also play a role in the longitudinal variation of the equatorial electrojet.

Satellite measurements have also provided useful information about the longitudinal dependence of the equatorial electrojet intensity. Early satellite observations showed diverse results (Cain and Sweeney 1973; Onwumechili and Agu 1981; Langel et al. 1993; Kim and King 1999; Jadhav et al. 2002; Ivers et al. 2003; Lühr et al. 2004). More recent studies have established that the longitudinal variation of the daytime equatorial electrojet intensity is often dominated by the so-called "wave-4" pattern with four peaks and four troughs between $0^{\circ}$ and $360^{\circ}$ longitudes (Le Mouël et al. 2006; England et al. 2006; Alken and Maus 2007; Lühr et al. 2008). Figure 56b (from Lühr and Manoj 2013) displays the longitudinal dependence of the equatorial electrojet intensity. The wave- 4 longitudinal pattern is most prominent during June-October. A similar longitudinal pattern has been found in the equatorial vertical plasma drift velocity (Kil et al. 2007; 
a.

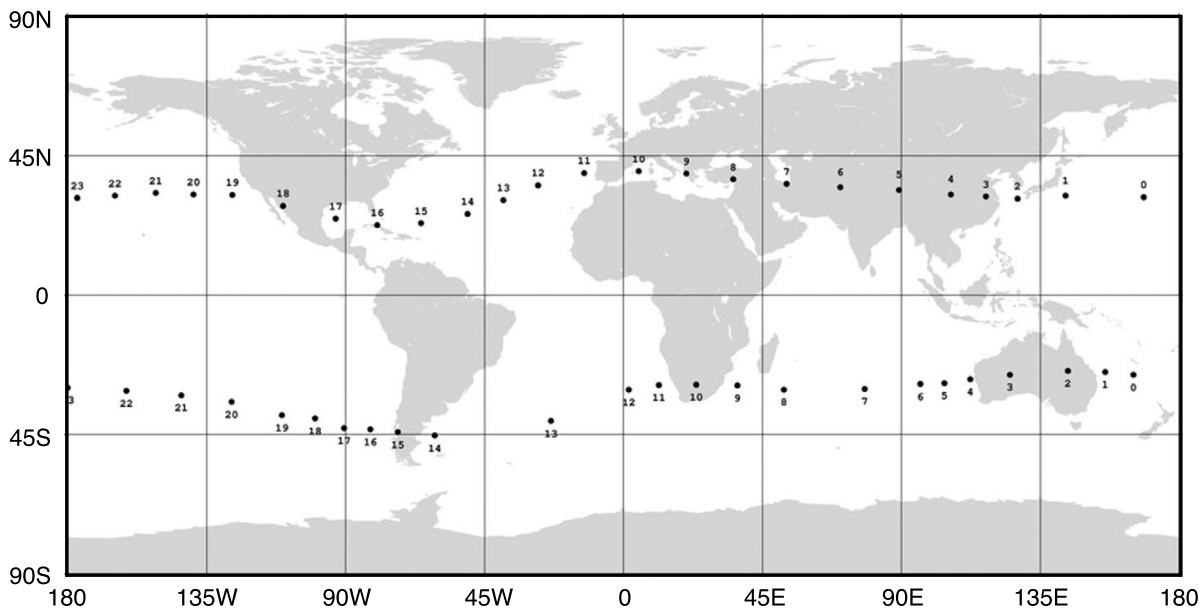

b.

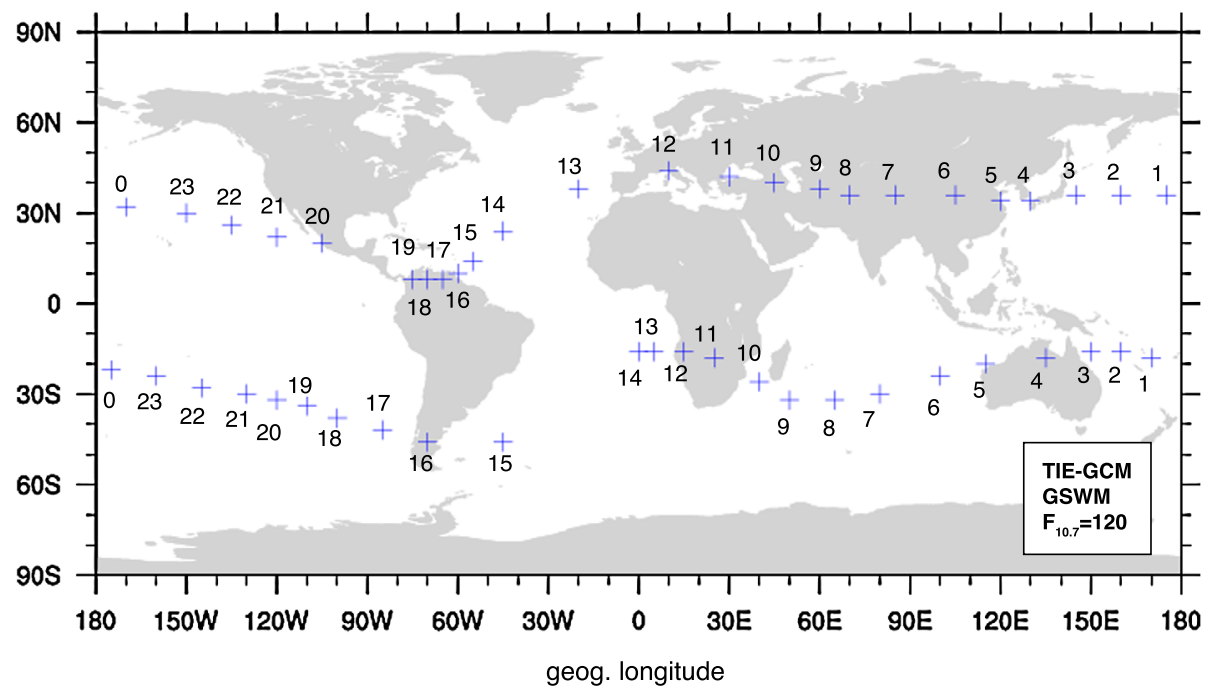

Fig. 55 The position of the northern and southern Sq current foci at different universal times, derived from (a) ground-based magnetometer measurements by Çelik (2013) and (b) TIE-GCM with $F_{10.7}=120$ sfu

Fejer et al. 2008). Numerical studies have shown that the eastward-propagating diurnal tide with wave number 3 , the so-called DE3, is the main cause of the wave-4 longitudinal pattern in the ionosphere (Hagan et al., 2007; Jin et al., 2008). The DE3 is primarily excited by latent heating due to deep tropical convection in the troposphere. At dynamo-region heights, the DE3 amplitude undergoes a similar seasonal variation as the wave-4 pattern in the equatorial electrojet intensity (Forbes et al. 2008). Figure 56c shows the results of the TIE-GCM simulation that takes into account the effect of the DE3. The TIE-GCM results clearly show the wave-4 longitudinal pattern, consistent with the satellite measurements. 
a.

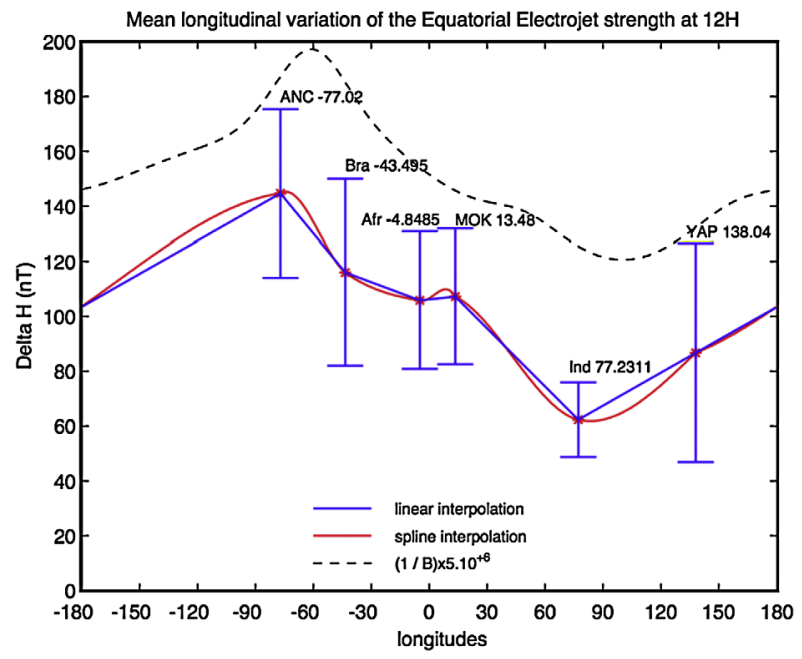

b.
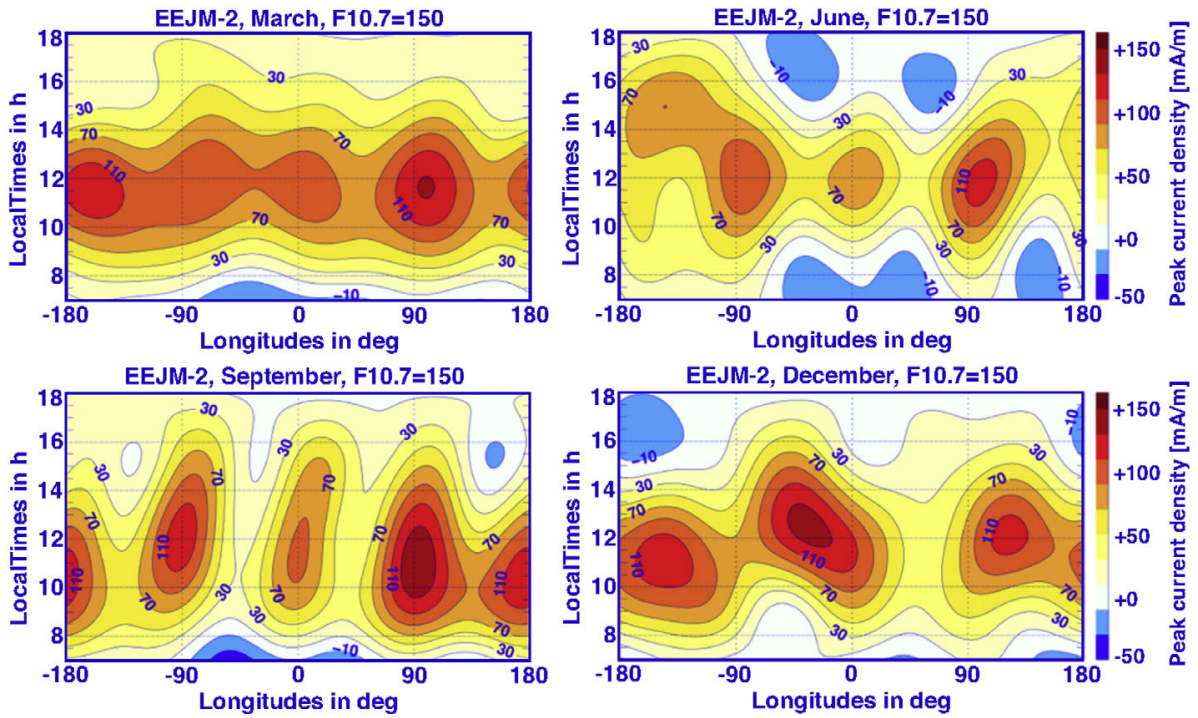

Fig. 56 The longitudinal dependence of the noon-time equatorial electrojet intensity, derived from (a) ground-based magnetometer data during the IEEY; (b) the satellite-based empirical model of Alken and Maus (2007); and (c) TIE-GCM with $F_{10.7}=120$ sfu. Panels (a) and (b) are from Doumouya et al. (2003) and Lühr and Manoj (2013), respectively

Obvious differences exist between the results obtained from ground and satellite magnetometers (i.e., Figs. 56a and 56b). This is likely to be due to the limited ability of ground magnetometers to assess the longitudinal dependence of the equatorial electrojet. It is difficult to resolve the wave-4 pattern using sparsely distributed ground magnetometers near the magnetic equator. Also, ground equatorial stations are not exactly at the magnetic equator, which adds to the uncertainty of the equatorial electrojet intensity evaluated at individual stations. 

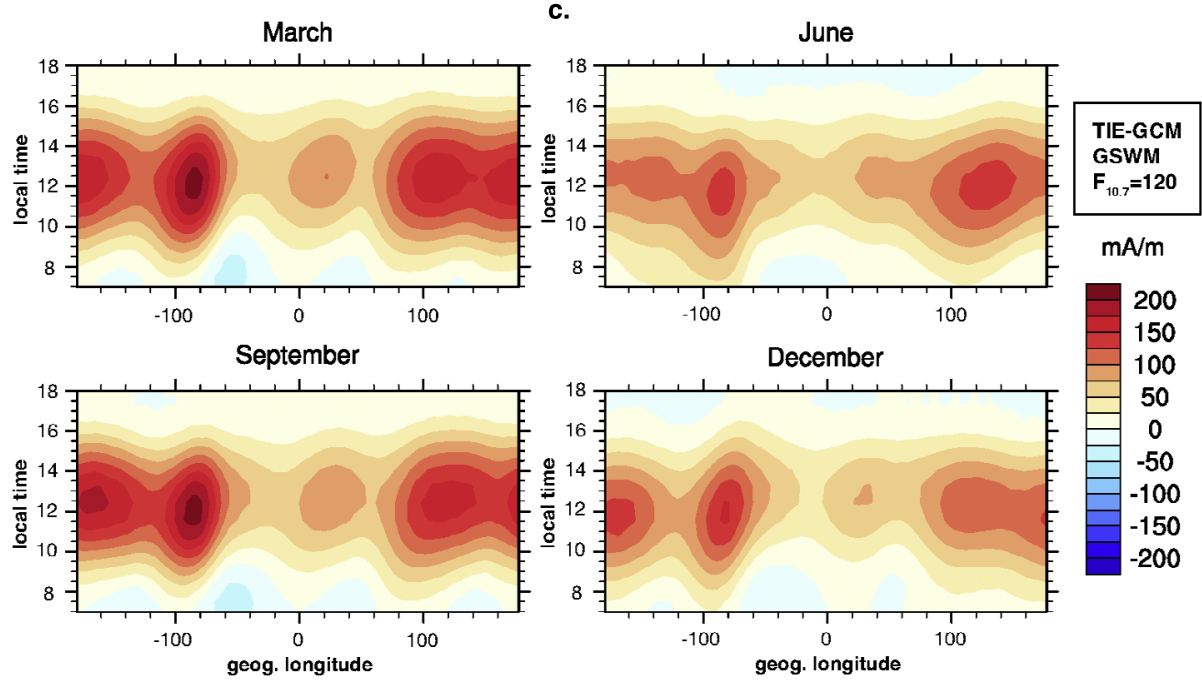

Fig. 56 (Continued)

\section{Remaining Questions}

Considerable progress has been made in understanding of Sq and EEJ since the time of Chapman and Bartels (1940). As we have seen above, there are many unsolved issues that require further work. Below, we itemize these remaining questions with reference to the section (and literature) that addresses some aspect of the issue.

- How much are $\mathrm{Sq}$ variations on the ground contaminated by the effect of interhemispheric field-aligned currents (Sect. 2.2)? Richmond and Roble (1987), using a numerical model, separated the equivalent $\mathrm{Sq}$ current system into two components; one associated with horizontal ionospheric currents and the other associated with interhemispheric field-aligned currents. Their prediction needs to be validated using experimental data.

- Large-amplitude counter electrojet events during the northern winter are often associated with a stratospheric sudden warming (Sects. 2.2 and 4.3.3). What is the driving mechanism for the counter electrojet events that occur during other periods of year? Can we associate them with processes in the lower and middle atmosphere?

- How does the strong eastward flow of the equatorial electrojet close (Sect. 2.5)? How much does the return flow of the equatorial electrojet account for the Sq variations at low and middle latitudes?

- Does the pre-reversal enhancement of the zonal equatorial electric field produce any magnetic signal on the ground (Sect. 3.11)?

- Why do the Sq current foci and peak equatorial electrojet shift to later local times during solar maximum (Sects. 4.1.2 and 4.1.3)?

- What causes the difference between the year-to-year variations in Sq and L (Sect. 4.1.1; Fig. 33)?

- Is it possible to estimate day-to-day changes in the neutral wind using magnetometer data (Sect. 4.3.1)? Numerical studies have shown that day-to-day variations in the Sq currents and equatorial electrojet arise from irregular changes in the neutral wind due to forcing 
from the lower atmosphere (Yamazaki et al. 2014a, 2016). Gurubaran et al. (2011) showed that the correlation sometimes exists between geomagnetic variations and mesospheric winds in the Indian sector. More comprehensive studies will be necessary to establish the empirical relations.

- What is the spatial pattern of the equivalent ionospheric current systems associated with 2-, 5-, 10-, and 16-day geomagnetic variations (Sect. 4.3.2)? This question has been only partially addressed for 2-day geomagnetic variations by Yamada (2009).

- The relationship between the occurrence of stratospheric sudden warmings and largeamplitude lunar variation in the equatorial electrojet has been established (Sect. 4.3.3). Why are there winters when the lunar variation in the equatorial electrojet is enhanced without a stratospheric sudden warming, and why are there winters when the lunar variation in the equatorial electrojet is not enhanced during a stratospheric sudden warming?

- Numerical studies have shown that the disturbance dynamo causes ionospheric currents that oppose to the normal Sq currents. Is the global anti-Sq current system observable? Le Huy and Amory-Mazaudier (2008) reported on a reduced Sq current system during the recovery phase of the storm on 25 November 2001. However, such a reduction can occur under quiet conditions due to irregular changes in the neutral wind. Statistical studies will be necessary, which are so far limited to the equatorial region only (Fejer and Scherliess 1995; Yamazaki and Kosch 2015).

- Do ionospheric currents flow on the nightside during disturbed periods? The NCAR TIEGCM predicts that the penetration electric field from high latitudes gives rise to nighttime currents at midlatitudes (Sect. 4.3.4; Fig. 49). Experimental evidence is desirable.

- How do some solar flares cause a reduction in the eastward flow of the equatorial electrojet (Sect. 4.4; Fig. 51)? What is the direction of the zonal electric field during such an event?

- Can geomagnetic solar eclipse effects be observed at conjugate points, as predicted by Takeda and Araki (1984b) (Sect. 4.4)?

- Longitudinal variations of the Sq current intensity observed by ground and satellite magnetometers are not consistent (Sect. 4.6.1; Fig. 54). What causes these discrepancies?

\section{List of Data Sources}

The following is a list of data sources used in the present paper:

(1) Hourly geomagnetic data (Figs. 1, 15, 18, 19, 27, 30, 31, 32, and 42)

World Data Center for Geomagnetism, Kyoto

[http://wdc.kugi.kyoto-u.ac.jp/hyplt/index.html]

World Data Center for Geomagnetism, Edinburgh

[http://www.wdc.bgs.ac.uk/catalog/master.html]

(2) Hourly solar wind data (Fig. 1)

OMNIWeb (NASA/GSFC's Space Physics Data Facility)

[http://omniweb.gsfc.nasa.gov/ow.html]

(3) $K p$ index and IQDs (Figs. 1, 15, 18, and 19)

GFZ German Research Centre for Geosciences

[http://www.gfz-potsdam.de/en/kp-index/]

(4) Ionopsheric conductivity model (Fig. 7)

World Data Center for Geomagnetism, Kyoto

[http://wdc.kugi.kyoto-u.ac.jp/ionocond/exp/icexp.html] 
(5) NCAR TIE-GCM (Figs. 8, 9, 49, 54, 55, and 56)

High Altitude Observatory, National Center for Atmospheric Research

[http://www.hao.ucar.edu/modeling/tgcm/]

(6) Hourly Dst index (Figs. 18 and 19)

World Data Center for Geomagnetism, Kyoto

[http://wdc.kugi.kyoto-u.ac.jp/dstae/index.html]

(7) Sunspot number (Fig. 30)

Sunspot Index and Long-term Solar Observations

[http://www.sidc.be/silso/datafiles]

(8) $F_{10.7}$ solar activity index (Figs. 30, 31, and 32)

OMNIWeb (NASA/GSFC's Space Physics Data Facility)

[http://omniweb.gsfc.nasa.gov/form/dx1.html]

(9) MgII solar activity index (Fig. 30)

University of Bremen

[http://www.iup.uni-bremen.de/gome/gomemgii.html]

(10) Solar EUV flux (Fig. 30)

Space Science Center, University of Southern California

[http://www.usc.edu/dept/space_science/sem_data/sem_data.html]

Acknowledgements The hourly geomagnetic data used in this paper were provided by the World Data Centers at Kyoto and Edinburgh. We thank them for maintaining archives of the data. We also thank the individual observatories (Alibag, Hermanus, Honolulu, Huancayo, Jaipur, Kakioka, Kashi, Memambetsu, Novosibirsk, San Juan, Tirunelveli, and Trivandrum) for the operation. We thank Dr. A.D. Richmond for his comments on a draft manuscript. We thank Dr. C. Çelik for providing us with the plots we requested. Y.Y. was supported by Natural Environment Research Council Grant NE/K01207X/1. A.M. was supported by NSF award AGS 1135446. The National Center for Atmospheric research is sponsored by the National Science Foundation.

Open Access This article is distributed under the terms of the Creative Commons Attribution 4.0 International License (http://creativecommons.org/licenses/by/4.0/), which permits unrestricted use, distribution, and reproduction in any medium, provided you give appropriate credit to the original author(s) and the source, provide a link to the Creative Commons license, and indicate if changes were made.

\section{References}

M.A. Abdu, The international equatorial electrojet year. Eos Trans. AGU 73(5), 49-54 (1992). doi:10.1029/ $91 \mathrm{EO} 00044$

N.S. Abdul Hamid, H. Liu, T. Uozumi, K. Yumoto, B. Veenadhari, A. Yoshikawa, J.A. Sanchez, Relationship between the equatorial electrojet and global Sq currents at the dip equator region. Earth Planets Space 66, 146 (2014). doi:10.1186/s40623-014-0146-2

N.S. Abdul Hamid, H. Liu, T. Uozumi, A. Yoshikawa, Empirical model of equatorial electrojet based on ground-based magnetometer data during solar minimum in fall. Earth Planets Space 67, 205 (2015). doi:10.1186/s40623-015-0373-1

S. Alex, B.D. Kadam, D.R.K. Rao, Ionospheric current systems on days of low equatorial $\Delta H$. J. Atmos. Sol.-Terr. Phys. 60, 371-379 (1998)

P. Alken, S. Maus, Spatio-temporal characterization of the equatorial electrojet from CHAMP, Ørsted, and SAC-C satellite magnetic measurements. J. Geophys. Res. 112, A09305 (2007). doi:10.1029/ 2007JA012524

P. Alken, S. Maus, Relationship between the ionospheric eastward electric field and the equatorial electrojet. Geophys. Res. Lett. 37, L04104 (2010). doi:10.1029/2009GL041989

P. Alken, A. Chulliat, S. Maus, Longitudinal and seasonal structure of the ionospheric equatorial electric field. J. Geophys. Res. Space Phys. 118, 1298-1305 (2013). doi:10.1029/2012JA018314

P. Alken, S. Maus, A. Chulliat, P. Vigneron, O. Sirol, G. Hulot, Swarm equatorial electric field chain: first results. Geophys. Res. Lett. 42, 673-680 (2015). doi:10.1002/2014GL062658 
D. Anderson, Daytime vertical $\mathrm{E} \times \mathrm{B}$ drift velocities inferred from ground-based equatorial magnetometer observations, in Aeronomy of the Earth's Atmosphere and Ionosphere (Springer, Netherlands, 2011), pp. 203-210

D. Anderson, A. Anghel, K. Yumoto, M. Ishitsuka, E. Kudeki, Estimating daytime vertical E $\times$ B drift velocities in the equatorial F-region using ground-based magnetometer observations. Geophys. Res. Lett. 29, 1596 (2002)

D. Anderson, A. Anghel, J.L. Chau, O. Veliz, Daytime vertical $\mathrm{E} \times \mathrm{B}$ drift velocities inferred from groundbased magnetometer observations at low latitudes. Space Weather 2(S11001) (2004). doi:10.1029/ 2004SW000095

D. Anderson, A. Anghel, J.L. Chau, K. Yumoto, Global, low-latitude, vertical E $\times$ B drift velocities inferred from daytime magnetometer observations. Space Weather 4, S08003 (2006). doi:10.1029/ 2005SW000193

D. Andrews, J.R. Holton, C.B. Leovy, Stratospheric sudden warmings, in Middle Atmosphere Dynamics (Elsevier, New York, 1987), pp. 259-294

A. Anghel, D. Anderson, N. Maruyama, J. Chau, K. Yumoto, A. Bhattacharyya, S. Alex, Interplanetary electric fields and their relationship to low-latitude electric fields under disturbed conditions. J. Atmos. Sol.-Terr. Phys. 69, 1147-1159 (2007)

E.V. Appleton, M.A.F. Barnett, Local reflection of wireless waves from the upper atmosphere. Nature 115, 333-334 (1925)

W.G. Baker, D.F. Martyn, Electric currents in the ionosphere, I: the conductivity. Philos. Trans. R. Soc. Lond. Ser. A 246, 281-294 (1953)

N. Balan, K.N. Iyer, Equatorial anomaly in ionospheric electron content and its relation to dynamo currents. J. Geophys. Res. 88, 10259-10262 (1983)

B.B. Balsley, R.F. Woodman, On the control of the F-region drift velocity by the E-region electric field: experimental evidence. J. Atmos. Terr. Phys. 31, 865-867 (1969)

J. Bartels, H.F. Johnston, Geomagnetic tides in horizontal intensity at Huancayo. Terr. Magn. Atmos. Electr. 45, 269-308 (1940)

S.K. Bhardwaj, P.B.V. Subba Rao, B. Veenadhari, Abnormal quiet day variations in Indian region along $75^{\circ}$ E meridian. Earth Planets Space 67, 115 (2015). doi:10.1186/s40623-015-0292-1

B.N. Bhargava, N.S. Sastri, A comparison of days with and without occurrence of counter electrojet afternoon events in the Indian region. Ann. Geophys. 33, 329-333 (1977)

B.N. Bhargava, D.R.K. Rao, B.R. Arora, Semi-annual modulation of earth's magnetic field in the equatorial electrojet region. Planet. Space Sci. 21, 1251-1255 (1973)

A. Bhattacharyya, K.C. Okpala, Principal components of quiet time temporal variability of equatorial and low-latitude geomagnetic fields. J. Geophys. Res. Space Phys. 120, 8799-8809 (2015). doi:10.1002/ 2015JA021673

M. Blanc, A.D. Richmond, The ionospheric disturbance dynamo. J. Geophys. Res. 85(A4), 1669-1686 (1980)

O.S. Bolaji, A.B. Rabiu, O.R. Bello, A. Yoshikawa, K. Yumoto, O.O. Odeyemi, O. Ogunmodimu, Spatial variability of solar quiet fields along $96^{\circ}$ magnetic meridian in Africa: results from MAGDAS. J. Geophys. Res. Space Phys. 120, 3883-3898 (2015). doi:10.1002/2014JA020728

H.A. Bomke, H.A. Blake, A.K. Harris, W.H. Hulse, D.J. Sheppard, A.A. Giesecke, A. Pantoja, Recombination coefficient and coronal contribution to E-layer ionization from magnetic observations of a solar eclipse. J. Geophys. Res. 72(23), 5913-5918 (1967)

G. Breit, M.A. Tuve, A radio method of estimating the height of the conducting layer. Nature 116, 357 (1925)

J. Bremer, R. Schminder, K.M. Greisiger, P. Hoffmann, D. Kurschner, W. Singer, Solar cycle dependence and long-term trends in the wind field of the mesosphere/lower thermosphere. J. Atmos. Sol.-Terr. Phys. 59, 497-509 (1997)

B.H. Briggs, The variability of ionospheric dynamo currents. J. Atmos. Terr. Phys. 46, 419-429 (1984)

G.M. Brown, W.R. Williams, Some properties of the day-to-day variability of $\mathrm{Sq}(\mathrm{H})$. Planet. Space Sci. 17, 455-470 (1969)

W.J. Burke, L.C. Gentile, C.Y. Huang, C.E. Valladares, S.Y. Su, Longitudinal variability of equatorial plasma bubbles observed by DMSP and ROCSAT-1. J. Geophys. Res. 109, A12301 (2004). doi:10.1029/ 2004JA010583

E.C. Butcher, On the location of the ionospheric current systems responsible for the lunar and solar magnetic variations. Geophys. J. R. Astron. Soc. 63, 175-782 (1980)

L.J. Cahill Jr., Investigation of the equatorial electrojet by rocket magnetometer. J. Geophys. Res. 64(5), 489-503 (1959). doi:10.1029/JZ064i005p00489

J. Cain, R. Sweeney, The POGO data. J. Atmos. Terr. Phys. 35, 1231-1247 (1973)

W.H. Campbell, Occurrence of AE and Dst geomagnetic index levels and the selection of the quietest days in a year. J. Geophys. Res. 84(A3), 875-881 (1979). doi:10.1029/JA084iA03p00875 
W.H. Campbell, Annual and semiannual variations of the lunar semidiurnal geomagnetic field components at North American locations. J. Geomagn. Geoelectr. 32, 105-128 (1980)

W.H. Campbell, Annual and semiannual variations of the geomagnetic field at equatorial locations. J. Atmos. Terr. Phys. 43, 607-616 (1981)

W.H. Campbell, Annual and semiannual changes of the quiet daily variations (Sq) in the geomagnetic field at North American locations. J. Geophys. Res. 87, 785-796 (1982)

W.H. Campbell, Some effects of quiet geomagnetic field changes upon values for main field modeling. Phys. Earth Planet. Inter. 48, 193-199 (1987)

W.H. Campbell, The regular geomagnetic-field variations during quiet solar conditions, in Geomagnetism, vol. 3 (1989a), pp. 385-460

W.H. Campbell, An introduction to quiet daily geomagnetic fields. Pure Appl. Geophys. 131, 315-331 (1989b)

W.H. Campbell, Differences in geomagnetic Sq field representations due to variations in spherical harmonic analysis techniques. J. Geophys. Res. 95(A12), 20923-20936 (1990). doi:10.1029/JA095iA12p20923

W.H. Campbell, Introduction to Geomagnetic Fields (Cambridge Univ. Press, Cambridge, 2003)

W.H. Campbell, Failure of Dst index fields to represent a ring current. Space Weather 2, S08002 (2004)

W.H. Campbell, S. Matsushita, Sq currents: a comparison of quiet and active year behavior. J. Geophys. Res. 87(A7), 5305-5308 (1982). doi:10.1029/JA087iA07p05305

W.H. Campbell, E.R. Schiffmacher, Quiet ionospheric currents of the northern hemisphere derived from geomagnetic field records. J. Geophys. Res. 90(A7), 6475-6486 (1985). doi:10.1029/JA090iA07p06475

W.H. Campbell, E.R. Schiffmacher, Correction to "Quiet ionospheric currents of the northern hemisphere derived from geomagnetic field records". J. Geophys. Res. 91(8A), 9023-9024 (1986). doi:10.1029/JA091iA08p09023

W.H. Campbell, E.R. Schiffmacher, Upper mantle electrical conductivity for seven subcontinental regions of the Earth. J. Geomagn. Geoelectr. 40, 1387-1406 (1988a)

W.H. Campbell, E.R. Schiffmacher, Quiet ionospheric currents of the southern hemisphere derived from geomagnetic records. J. Geophys. Res. 93(A2), 933-944 (1988b). doi:10.1029/JA093iA02p00933

W.H. Campbell, E.R. Schiffmacher, H.W. Kroehl, Global quiet day field variation model WDCA/SQ1. Eos Trans. AGU 70(5), 66-74 (1989). doi:10.1029/89EO00039

W.H. Campbell, E.R. Schiffmacher, B.R. Arora, Quiet geomagnetic field representation for all days and latitudes. J. Geomagn. Geoelectr. 44, 459-480 (1992)

W.H. Campbell, B.R. Arora, E.R. Schiffmacher, External Sq currents in the India-Siberia region. J. Geophys. Res. 98(A3), 3741-3752 (1993). doi:10.1029/92JA02552

W.H. Campbell, C.E. Barton, F.H. Chamalaun, W. Welsh, Quiet-day ionospheric currents and their application to upper mantle conductivity in Australia. Earth Planets Space, vol. 50 (1998) pp. 347-360

C. Çelik, The solar daily geomagnetic variation and its dependence on sunspot number. J. Atmos. Sol.-Terr. Phys. 104, 75-86 (2013)

C. Çelik, The lunar daily geomagnetic variation and its dependence on sunspot number. J. Atmos. Sol.-Terr. Phys. 119, 153-161 (2014)

C. Çelik, M.K. Tunçer, E. Tolak-Çiftçi, M. Zobu, N. Oshiman, S.B. Tank, Solar and lunar geomagnetic variations in the northwestern part of Turkey. Geophys. J. Int. 189, 391-399 (2012)

F.H. Chamalaun, C.E. Barton, The large-scale electrical conductivity structure of Australia. J. Geomagn. Geoelectr. 45, 1209-1212 (1993)

H. Chandra, R.K. Misra, R.G. Rastogi, Equatorial ionospheric drift and the electrojet. Planet. Space Sci. 19, 1497-1503 (1971)

L.C. Chang, S.E. Palo, H.-L. Liu, Short-term variability in the migrating diurnal tide caused by interactions with the quasi 2 day wave. J. Geophys. Res. 116, D12112 (2011). doi:10.1029/2010JD014996

S. Chapman, The solar and lunar diurnal variations of terrestrial magnetism. Philos. Trans. R. Soc. A 218, $1-118$ (1919)

S. Chapman, The effect of a solar eclipse on the Earth's magnetic field. Terr. Magn. 38, 176-183 (1933)

S. Chapman, The equatorial electrojet as detected from the abnormal electric current distribution above Huancayo, Peru, and elsewhere. Arch. Meteorol. Geophys. Bioklimatol. Ser. A. 4, 368-390 (1951)

S. Chapman, J. Bartels, Geomagnetism (Oxford Univ. Press, London, 1940)

S. Chapman, J.C.P. Miller, The statistical determination of lunar daily variations in geomagnetic and meteorological elements. Mon. Not. R. Astron. Soc. 4, 649-669 (1940)

S. Chapman, K.R. Raja Rao, The $\mathrm{H}$ and $\mathrm{Z}$ variations along and near the equatorial electrojet in India, Africa and the Pacific. J. Atmos. Terr. Phys. 27, 559-581 (1965)

S. Chapman, J.C. Gupta, S.R.C. Malin, The sunspot cycle influence on the solar and lunar daily geomagnetic variations. Proc. R. Soc. Lond. A 324 (1971). doi:10.1098/rspa.1971.0124

J.L. Chau, B.G. Fejer, L.P. Goncharenko, Quiet variability of equatorial E $\times$ B drifts during a sudden stratospheric warming event. Geophys. Res. Lett. 36, L05101 (2009). doi:10.1029/2008GL036785 
J.L. Chau, P. Hoffmann, N.M. Pedatella, V. Matthias, G. Stober, Upper mesospheric lunar tides over middle and high latitudes during sudden stratospheric warming events. J. Geophys. Res. Space Physics 120 (2015). doi:10.1002/2015JA020998

P.-R. Chen, Y. Luo, J. Ma, The QBO modulation fo the occurrence of the counter electrojet. Geophys. Res. Lett. 22, 2717-2720 (1995)

G.-X. Chen, W.-Y. Xu, A.-M. Du, Y.-Y. Wu, B. Chen, X.-C. Liu, Statistical characteristics of the dayto-day variability in the geomagnetic Sq field. J. Geophys. Res. 112, A06320 (2007). doi:10.1029/ 2006JA012059

C.H. Chen, J.Y. Liu, K. Yumoto, C.H. Lin, T.W. Fang, Equatorial ionization anomaly of the total electron content and equatorial electrojet of ground-based geomagnetic field strength. J. Atmos. Sol.-Terr. Phys. 70, 2172-2183 (2008)

R.K. Choudhary, J.-P. St.-Maurice, K.M. Ambili, S. Sunda, B.M. Pathan, The impact of the January 15, 2010, annular solar eclipse on the equatorial and low latitude ionospheric densities. J. Geophys. Res. 116, A09309 (2011). doi:10.1029/2011JA016504

A. Chulliat, E. Blanter, J.-L. Le Mouël, M. Shnirman, On the seasonal asymmetry of the diurnal and semidiurnal geomagnetic variations. J. Geophys. Res. 110, A05301 (2005). doi:10.1029/2004JA010551

A. Chulliat, P. Vigneron, E. Thébault, O. Sirol, G. Hulot, Swarm SCARF dedicated ionospheric field inversion chain. Earth Planets Space 65, 1271-1283 (2013). doi:10.5047/eps.2013.08.006

A. Chulliat, P. Vigneron, G. Hulot, First results from the swarm dedicated ionospheric field inversion chain. Earth Planets Space 68, 104 (2016). doi:10.1186/s40623-016-0481-6

E.W. Cliver, W.F. Dietrich, The 1859 space weather event revisited: limits of extreme activity. J. Space Weather Space Clim. 3, A31 (2013). doi:10.1051/swsc/2013053

E.W. Cliver, L. Svalgaard, The 1859 solar-terrestrial disturbance and the current limits of extreme space weather activity. Sol. Phys. 224, 407-422 (2004)

E.W. Cliver, Y. Kamide, A.G. Ling, Mountains versus valleys: semiannual variation of geomagnetic activity. J. Geophys. Res. 105(A2), 2413-2424 (2000). doi:10.1029/1999JA900439

I. Cnossen, Climate change in the upper atmosphere, in Greenhouse Gases: Emission, Measurement, and Management, ed. by G. Liu (InTech, Rijeka, 2012), pp. 315-336

I. Cnossen, A.D. Richmond, Changes in the Earth's magnetic field over the past century: effects on the ionosphere-thermosphere system and solar quiet (Sq) magnetic variation. J. Geophys. Res. Space Phys. 118, 849-858 (2013). doi:10.1029/2012JA018447

T.G. Cowling, The electrical conductivity of an ionized gas in the presence of a magnetic field. Mon. Not. R. Astron. Soc. 93, 90-98 (1933)

A.L. Cullington, Geomagnetic effects of the solar eclipse, 12 October 1958, at Apia, Western Samoa. N.Z. J. Geol. Geophys. 5(3), 499-507 (1962)

J.J. Curto, C. Amory-Mazaudier, J.M. Torta, M. Menvielle, Solar flare effects at Ebre: regular and reversed solar flare effects, statistical analysis (1953 to 1985), a global case study and a model of elliptical ionospheric currents. J. Geophys. Res. 99(A3), 3945-3954 (1994). doi:10.1029/93JA02270

T.N. Davis, K. Burrows, J.D. Stolarik, A latitude survey of the equatorial electrojet with rocket-borne magnetometers. J. Geophys. Res. 72, 1845-1861 (1967)

K.A. Day, R.E. Hibbins, N.J. Mitchell, Aura MLS observations of the westward-propagating $s=1$, 16-day planetary wave in the stratosphere, mesosphere and lower thermosphere. Atmos. Chem. Phys. 11, 41494161 (2011)

B.F. de Haro Barbas, A.G. Elias, I. Cnossen, M. Zossi de Artigas, Long-term changes in solar quiet (Sq) geomagnetic variations related to Earth's magnetic field secular variation. J. Geophys. Res. Space Phys. 118, 3712-3718 (2013). doi:10.1002/jgra.50352

V. Doumouya, J. Vassal, Y. Cohen, O. Fambitakoye, M. Menvielle, Equatorial electrojet at African longitudes: first results from magnetic measurements. Ann. Geophys. 16, 658-676 (1998)

V. Doumouya, Y. Cohen, B.R. Arora, K. Yumoto, Local time and longitude dependence of the equatorial electrojet magnetic effects. J. Atmos. Sol.-Terr. Phys. 65, 1265-1282 (2003)

J. Du, R.J. Stening, Simulating the ionospheric dynamo, II: equatorial electric fields. J. Atmos. Sol.-Terr. Phys. 61, 925-940 (1999)

E. Dunford, The relationship between the ionospheric equatorial anomaly and the E-region current system. J. Atmos. Terr. Phys. 29, 1489-1498 (1967)

R. El Hawary, K. Yumoto, Y. Yamazaki, A. Mahrous, E. Ghamry, A. Meloni, K. Badi, G. Kianji, C.B.S. Uiso, N. Mwiinga, L. Joao, T. Affluo, P.R. Sutcliffe, G. Mengistu, P. Baki, S. Abe, A. Ikeda, A. Fujimoto, T. Tokunaga, Annual and semi-annual Sq variations at $96^{\circ}$ MM MAGDAS I and II stations in Africa. Earth Planets Space 64, 425-432 (2012)

A.G. Elias, M. Zossi de Artigas, B.F. de Haro Barbas, Trends in the solar quiet geomagnetic field variation linked to the Earth's magnetic field secular variation and increasing concentrations of greenhouse gases. J. Geophys. Res. 115, A08316 (2010). doi:10.1029/2009JA015136 
W. Ellis, On the relation between magnetic disturbance and the period of solar spot frequency. Proc. R. Soc. Lond. 63, 64-78 (1898)

S.L. England, S. Maus, T.J. Immel, S.B. Mende, Longitudinal variation of the E-region electric fields caused by atmospheric tides. Geophys. Res. Lett. 33, L21105 (2006). doi:10.1029/2006GL027465

O. Fambitakoye, P.N. Mayaud, Equatorial electrojet and regular daily variation $S_{R}$, I: a determination of the equatorial electrojet parameters. J. Atmos. Terr. Phys. 38, 1-17 (1976a)

O. Fambitakoye, P.N. Mayaud, Equatorial electrojet and regular daily variation $S_{R}$, II: the centre of the equatorial electrojet. J. Atmos. Terr. Phys. 38, 19-26 (1976b)

T.-W. Fang, A.D. Richmond, J.Y. Liu, A. Maute, C.H. Lin, C.H. Chen, B. Harper, Model simulation of the equatorial electrojet in the Peruvian and Philippine sectors. J. Atmos. Sol.-Terr. Phys. 70, 2203-2211 (2008)

T.-W. Fang, R. Akmaev, T. Fuller-Rowell, F. Wu, N. Maruyama, G. Millward, Longitudinal and day-to-day variability in the ionosphere from lower atmosphere tidal forcing. Geophys. Res. Lett. 40, 2523-2528 (2013). doi:10.1002/grl.50550

T.-W. Fang, T. Fuller-Rowell, H. Wang, R. Akmaev, F. Wu, Ionospheric response to sudden stratospheric warming events at low and high solar activity. J. Geophys. Res. Space Phys. 119, 7858-7869 (2014). doi:10.1002/2014JA020142

D.T. Farley, E. Bonelli, B.G. Fejer, M.F. Larsen, The prereversal enhancement of the zonal electric field in the equatorial ionosphere. J. Geophys. Res. 91(A12), 13723-13728 (1986). doi:10.1029/JA091iA12p13723

B.G. Fejer, L. Scherliess, Time dependent response of equatorial ionospheric electric fields to magnetospheric disturbances. Geophys. Res. Lett. 22(7), 851-854 (1995). doi:10.1029/95GL00390

B.G. Fejer, L. Scherliess, On the variability of equatorial F-region vertical plasma drifts. J. Atmos. Sol.-Terr. Phys. 63, 893-897 (2001)

B.G. Fejer, D.T. Farley, R.F. Woodman, C. Calderon, Dependence of equatorial F region vertical drifts on season and solar cycle. J. Geophys. Res. 84(A10), 5792-5796 (1979). doi:10.1029/JA084iA10p05792

B.G. Fejer, J.W. Jensen, S.-Y. Su, Quiet time equatorial F region vertical plasma drift model derived from ROCSAT-1 observations. J. Geophys. Res. 113, A05304 (2008). doi:10.1029/2007JA012801

B.G. Fejer, M.E. Olson, J.L. Chau, C. Stolle, H. Lühr, L.P. Goncharenko, K. Yumoto, T. Nagatsuma, Lunardependent equatorial ionospheric electrodynamic effects during sudden stratospheric warmings. J. Geophys. Res. 115, A00G03 (2010). doi:10.1029/2010JA015273

B.G. Fejer, B.D. Tracy, M.E. Olson, J.L. Cha, Enhanced lunar semidiurnal equatorial vertical plasma drifts during sudden stratospheric warmings. Geophys. Res. Lett. 38, L21104 (2011). doi:10.1029/ 2011GL049788

C.C. Finlay, N. Olsen, L. Toeffner-Claussen, DTU candidate field models for IGRF-12 and the CHAOS-5 geomagnetic field model. Earth Planets Space 67, 114 (2015). doi:10.1186/s40623-015-0274-3

J.M. Forbes, The equatorial electrojet. Rev. Geophys. 19(3), 469-504 (1981)

J.M. Forbes, S. Leveroni, Quasi 16-day oscillation in the ionosphere. Geophys. Res. Lett. 19, 981-984 (1991)

J.M. Forbes, R.S. Lindzen, Atmospheric solar tides and their electrodynamic effects, I: the global Sq current system. J. Atmos. Terr. Phys. 38, 897-910 (1976a)

J.M. Forbes, R.S. Lindzen, Atmospheric solar tides and their electrodynamic effects, II the equatorial electrojet. J. Atmos. Terr. Phys. 38, 911-920 (1976b)

J.M. Forbes, X. Zhang, Lunar tide amplification during the January 2009 stratosphere warming event: observations and theory. J. Geophys. Res. 117, A12312 (2012). doi:10.1029/2012JA017963

J.M. Forbes, X. Zhang, Quasi-10-day wave in the atmosphere. J. Geophys. Res., Atmos. 120, 11079-11089 (2015). doi:10.1002/2015JD023327

J.M. Forbes, X. Zhang, S. Palo, J. Russell, C.J. Mertens, M. Mlynczak, Tidal variability in the ionospheric dynamo region. J. Geophys. Res. 113, A02310 (2008). doi:10.1029/2007JA012737

J.M. Forbes, X. Zhang, S. Bruinsma, J. Oberheide, Lunar semidiurnal tide in the thermosphere under solar minimum conditions. J. Geophys. Res. Space Phys. 118, 1788-1801 (2013). doi:10.1029/ 2012JA017962

S.E. Forbush, M. Casaverde, Equatorial Electrojet in Peru (CIW, Washington, 1961)

N. Fukushima, Electric potential difference between conjugate points in middle latitudes caused by asymmetric dynamo in the ionosphere. J. Geomagn. Geoelectr. 31, 401-409 (1979)

N. Fukushima, Some topics and historical episodes in geomagnetism and aeronomy. J. Geophys. Res. 99, 19113-19142 (1994)

T.J. Fuller-Rowell, G.H. Millward, A.D. Richmond, M.V. Codrescu, Storm-time changes in the upper atmosphere at low latitudes. J. Atmos. Sol.-Terr. Phys. 64, 1383-1391 (2002)

T. Fuller-Rowell, F. Wu, R. Akmaev, T.-W. Fang, E. Araujo-Pradere, A whole atmosphere model simulation of the impact of a sudden stratospheric warming on thermosphere dynamics and electrodynamics. J. Geophys. Res. 115, A00G08 (2010). doi:10.1029/2010JA015524 
F. Gasperini, J.M. Forbes, Lunar-solar interactions in the equatorial electrojet. Geophys. Res. Lett. 41, 30263031 (2014). doi:10.1002/2014GL059294

C.F. Gauss, Allgemeine Theorie des Erdmagnetismus, Resultate aus den Beobachtungen des Magnetischen Vereins im Jahre 1838, in Scientific Memoirs Selected from the Transactions of Foreign Academies and Learned Societies and from Foreign Journals, ed. by C.F. Gauss, W. Weber (Taylor, London, 1841), pp. 184-251. English translation by Sabine, E. and Taylor, R.

L.R. Gaya-Piqué, J.J. Curto, J.M. Torta, A. Chulliat, Equivalent ionospheric currents for the 5 December 2006 solar flare effect determined from spherical cap harmonic analysis. J. Geophys. Res. 113, A07304 (2008). doi:10.1029/2007JA012934

J.W. Gjerloev, A global ground-based magnetometer initiative. Eos Trans. AGU 90, 230-231 (2009). doi:10.1029/2009EO270002

J.W. Gjerloev, The SuperMAG data processing technique. J. Geophys. Res. 117, A09213 (2012). doi:10.1029/ 2012JA017683

V.P. Golovkov, N.E. Papitashvili, I.S. Tiupkin, E.P. Kharin, Separation of geomagnetic field variations into quiet and disturbed components by the method of natural orthogonal components. Geomagn. Aeron. 18, 342-344 (1978)

L.P. Goncharenko, J.L. Chau, H.-L. Liu, A.J. Coster, Unexpected connections between the stratosphere and ionosphere. Geophys. Res. Lett. 37, L10101 (2010b). doi:10.1029/2010GL043125

L.P. Goncharenko, A.J. Coster, J.L. Chau, C.E. Valladares, Impact of sudden stratospheric warmings on equatorial ionization anomaly. J. Geophys. Res. 115, A00G07 (2010a). doi:10.1029/2010JA015400

L.P. Goncharenko, A.J. Coster, R.A. Plumb, D.I.V. Domeisen, The potential role of stratospheric ozone in the stratosphere-ionosphere coupling during stratospheric warmings. Geophys. Res. Lett. 39, L08101 (2012). doi:10.1029/2012GL051261

G. Graham, An account of observations made of the variation of the horizontal needle at London, in the latter part of the year 1722, and beginning of 1723. Philos. Trans. 33, 96-107 (1724a)

G. Graham, Observations of the dipping needle, made at London, in the beginning of the year 1723. Philos. Trans. 33, 332-339 (1724b)

J.D. Greener, D.M. Schlapp, A study of day-to-day variability of Sq over Europe. J. Atmos. Terr. Phys. 41, 217-223 (1979)

S.-Y. Gu, T. Li, X. Dou, Q. Wu, M.G. Mlynczak, J.M. Russell, Observations of Quasi-Two-Day wave by TIMED/SABER and TIMED/TIDI. J. Geophys. Res., Atmos. 118, 1624-1639 (2013). doi:10.1002/ jgrd.50191

J.C. Gupta, Movement of the Sq foci in 1958. Pure Appl. Geophys. 110, 2076-2084 (1973)

S. Gurubaran, The equatorial counter electrojet: Part of a worldwide current system? Geophys. Res. Lett. 29, 1337 (2002). doi:10.1029/2001GL014519

S. Gurubaran, T.K. Ramkumar, S. Sridharan, R. Rajaram, Signatures of quasi-2-day planetary waves in the equatorial electrojet: results from simultaneous observations of mesospheric winds and geomagnetic field variations at low latitudes. J. Atmos. Sol.-Terr. Phys. 63, 813-821 (2001)

S. Gurubaran, R. Dhanya, S. Sathiskumar, P.T. Patil, A case study of tidal and planetary wave coupling in the equatorial atmosphere-ionosphere system over India: preliminary results, in Aeronomy of the Earth's Atmosphere and Ionosphere (Springer, Netherlands, 2011), pp. 177-187

G.V. Haines, J.M. Torta, Determination of equivalent current sources from spherical cap harmonic models of geomagnetic field variations. Geophys. J. Int. 118, 499-514 (1994)

R. Hajra, S.K. Chakraborty, S. Mazumdar, S. Alex, Evolution of equatorial irregularities under varying electrodynamical conditions: a multitechnique case study from Indian longitude zone. J. Geophys. Res. 117, A08331 (2012). doi:10.1029/2012JA017808

C. Hanuise, C. Mazaudier, P. Vila, M. Blanc, M. Crochet, Global dynamo simulation of ionospheric currents and their connection with the equatorial electrojet and counter electrojet: a case study. J. Geophys. Res. 88, 253-270 (1983)

M. Hasegawa, On the position of the focus of the geomagnetic Sq current system. J. Geophys. Res. 65, 1437-1447 (1960)

R.A. Heelis, J.K. Lowell, R.W. Spiro, A model of the high-latitude ionospheric convection pattern. J. Geophys. Res. 87, 6339-6345 (1982)

F.H. Hibberd, Day-to-day variability of the Sq geomagnetic field variation. Aust. J. Phys. 34, 81-90 (1981)

F.H. Hibberd, The geomagnetic Sq variation-annual, semi-annual and solar cycle variations and ring current effects. J. Atmos. Terr. Phys. 47, 341-352 (1985)

M. Hirono, On the influence of the Hall current to the electrical conductivity of the ionosphere, I. J. Geomagn. Geoelectr. 2, 1-8 (1950)

A. Hollingsworth, The effect of ocean and Earth tides on the semi-diurnal lunar air tide. J. Atmos. Sci. 28, 1021-1044 (1971) 
Y.-N. Huang, K. Cheng, S.-W. Chen, On the equatorial anomaly of the ionospheric total electron content near the northern anomaly crest region. J. Geophys. Res. 94, 13515-13525 (1989)

C.-S. Huang, J.C. Foster, M.C. Kelley, Long-duration penetration of the interplanetary electric field to the lowlatitude ionosphere during the main phase of magnetic storms. J. Geophys. Res. 110, A11309 (2005b). doi:10.1029/2005JA011202

C.-M. Huang, A.D. Richmond, M.-Q. Chen, Theoretical effects of geomagnetic activity on low-latitude ionospheric electric fields. J. Geophys. Res. 110, A05312 (2005a). doi:10.1029/2004JA010994

R. Hutton, Sq currents in the American equatorial zone during the IGY, I: seasonal effects. J. Atmos. Terr. Phys. 29, 1411-1427 (1967)

M. Hvoždara, A. Prigancovă, Geomagnetic effects due to an eclipse-induced low-conductivity ionospheric spot. J. Geophys. Res. 107(A12), 1467 (2002). doi:10.1029/2002JA009260

R. Ito, S. Kato, T. Tsuda, Consideration of an ionospheric wind dynamo driven by a planetary wave with a two-day period. J. Atmos. Terr. Phys. 48, 1-13 (1986)

D. Ivers, R. Stening, J. Turner, D. Winch, Equatorial electrojet from Ørsted scalar magnetic field observations. J. Geophys. Res. 108(A2), 1061 (2003). doi:10.1029/2002JA009310

Ch. Jacobi, D. Kürschner, Long-term trends of MLT region winds over Central Europe. Phys. Chem. Earth 31, 16-21 (2006)

G. Jadhav, M. Rajaram, R. Rajaram, A detailed study of equatorial electrojet phenomenon using Ørsted satellite observations. J. Geophys. Res. 107(A8), 1175 (2002). doi:10.1029/2001JA000183

J. Jankowski, C. Sucksdorff, Guide for Magnetic Measurements and Observatory Practice (Int. Assoc. Geomagn. Aeron., Warsaw, 1996)

A.S. Janzhura, O.A. Troshichev, Determination of the running quiet daily geomagnetic variation. J. Atmos. Sol.-Terr. Phys. 70, 962-972 (2008)

M.J. Jarvis, Observed tidal variation in the lower thermosphere through the 20th century and the possible implication of ozone depletion. J. Geophys. Res. 110, A04303 (2005). doi:10.1029/2004JA010921

M.J. Jarvis, Planetary wave trends in the lower thermosphere: evidence for 22-year solar modulation of the quasi 5-day wave. J. Atmos. Sol.-Terr. Phys. 68, 1902-1912 (2006)

H. Jin, Y. Miyoshi, H. Fujiwara, H. Shinagawa, K. Terada, N. Terada, M. Ishii, Y. Otsuka, A. Saito, Vertical connection from the tropospheric activities to the ionospheric longitudinal structure simulated by a new Earth's whole atmosphere-ionosphere coupled model. J. Geophys. Res. 116, A01316 (2011). doi:10.1029/2010JA015925

H. Jin, Y. Miyoshi, D. Pancheva, P. Mukhtarov, H. Fujiwara, H. Shinagawa, Response of migrating tides to the stratospheric sudden warming in 2009 and their effects on the ionosphere studied by a whole atmosphere-ionosphere model GAIA with COSMIC and TIMED/SABER observations. J. Geophys. Res. 117, A10323 (2012). doi:10.1029/2012JA017650

Y. Kamide, Y.P. Maltsev, Geomagnetic storms, in Handbook of the Solar-Terrestrial Environment, ed. by Y. Kamide, A. Chian (Springer, Berlin, 2007), pp. 355-374

R.P. Kane, Relationship between H ranges at equatorial and middle latitudes. J. Atmos. Terr. Phys. 33, 319327 (1971)

R.P. Kane, Relation between the strength of the Sq current system and its focus position. Proc. Indian Acad. Sci. A 80, 17-25 (1974)

S. Kato, Horizontal wind systems in the ionospheric E region deduced from the dynamo theory of the geomagnetic Sq variation, II, Rotating earth. J. Geomagn. Geoelectr. 8, 24-37 (1956)

S. Kato, Horizontal wind systems in the ionospheric E region deduced from the dynamo theory of the geomagnetic Sq variation, IV. J. Geomagn. Geoelectr. 9, 107-115 (1957)

Y. Kato, The effect on the geomagnetic field of the solar eclipse of October 12, 1958. Science Reports of the Tohoku University 5th Ser. Geophys., 12.1 (1960)

S. Kato, Diurnal atmospheric oscillation, 1: eigenvalues and Hough functions. J. Geophys. Res. 71(13), 32013209 (1966). doi:10.1029/JZ071i013p03201

K. Kawano-Sasaki, S. Miyahara, A study on three-dimensional structures of the ionospheric dynamo currents induced by the neutral winds simulated by the Kyushu-GCM. J. Atmos. Sol.-Terr. Phys. 70, 1549-1562 (2008)

K. Kawasaki, S. Matsushita, J.C. Cain, Least squares and integral methods for the spherical harmonic analysis of the Sq field. Pure Appl. Geophys. 131, 357-370 (1989)

M.C. Kelley, J.J. Makela, J.L. Chau, M.J. Nicolls, Penetration of the solar wind electric field into the magnetosphere/ionosphere system. Geophys. Res. Lett. 30(4), 1158 (2003). doi:10.1029/2002GL016321

T. Kikuchi, H. Lühr, T. Kitamura, O. Saka, K. Schlegel, Direct penetration of the polar electric field to the equator during a DP 2 event as detected by the auroral and equatorial magnetometer chains and the EISCAT radar. J. Geophys. Res. 101(A8), 17161-17173 (1996). doi:10.1029/96JA01299

T. Kikuchi, H. Lühr, K. Schlegel, H. Tachihara, M. Shinohara, T.-I. Kitamura, Penetration of auroral electric fields to the equator during a substorm. J. Geophys. Res. 105(A10), 23251-23261 (2000). doi:10.1029/ 2000JA900016 
T. Kikuchi, K.K. Hashimoto, T.-I. Kitamura, H. Tachihara, B. Fejer, Equatorial counterelectrojets during substorms. J. Geophys. Res. 108(A11), 1406 (2003). doi:10.1029/2003JA009915

T. Kikuchi, K.K. Hashimoto, K. Nozaki, Penetration of magnetospheric electric fields to the equator during a geomagnetic storm. J. Geophys. Res. 113, A06214 (2008). doi:10.1029/2007JA012628

H. Kil, S.-J. Oh, M.C. Kelley, L.J. Paxton, S.L. England, E. Talaat, K.-W. Min, S.-Y. Su, Longitudinal structure of the vertical $\mathrm{E} \times \mathrm{B}$ drift and ion density seen from ROCSAT-1. Geophys. Res. Lett. 34, L14110 (2007). doi:10.1029/2007GL030018

H.R. Kim, S.D. King, A study of local time and longitudinal variability of the amplitude of the equatorial electrojet observed in POGO satellite data. Earth Planets Space 51, 373-381 (1999)

S. Koch, A. Kuvshinov, Global 3-D EM inversion of Sq variations based on simultaneous source and conductivity determination: concept validation and resolution studies. Geophys. J. Int. 195, 98-116 (2013)

S. Koch, A. Kuvshinov, 3-D EM inversion of ground based geomagnetic Sq data. Results from the analysis of Australian array (AWAGS) data. Geophys. J. Int. 200, 1284-1296 (2015)

A. Kohsiek, K.H. Glassmeier, T. Hirooka, Periods of planetary waves in geomagnetic variations. Ann. Geophys. 13, 168-176 (1995)

A.V. Kuvshinov, H. Utada, Anomaly of the geomagnetic Sq variation in Japan: effect from 3-D subterranean structure or the ocean effect? Geophys. J. Int. 183, 1239-1247 (2010). doi:10.1111/ j.1365-246X.2010.04809.x

A.V. Kuvshinov, D.B. Avdeev, O.V. Pankratov, Global induction by Sq and Dst sources in the presence of oceans: bimodal solutions for non-uniform spherical surface shells above radially symmetric earth models in comparison to observations. Geophys. J. Int. 137, 630-650 (1999)

A.V. Kuvshinov, C. Manoj, N. Olsen, T. Sabaka, On induction effects of geomagnetic daily variations from equatorial electrojet and solar quiet sources at low and middle latitudes. J. Geophys. Res. 112, B10102 (2007). doi:10.1029/2007JB004955

A.V. Ladynin, N.N. Semakov, S.Y. Khomutov, Changes in the daily geomagnetic variation during the total solar eclipse of 1. Russ. Geol. Geophys. 52, 343-352 (2008). 2011

R.A. Langel, M. Purucker, M. Rajaram, The equatorial electrojet and associated currents as seen in Magsat data. J. Atmos. Terr. Phys. 55, 1233-1269 (1993)

J. Laštovička, S.C. Solomon, L. Qian, Trends in the neutral ionized upper atmosphere. Space Sci. Rev. 168, 113-145 (2012)

A.R. Lawrence, M.J. Jarvis, Simultaneous observations of planetary waves from 30 to $220 \mathrm{~km}$. J. Atmos. Sol.-Terr. Phys. 65, 765-777 (2003)

M. Le Huy, C. Amory-Mazaudier, Magnetic signature of the ionospheric disturbance dynamo at equatorial latitudes: D dyn. J. Geophys. Res. 110, A10301 (2005). doi:10.1029/2004JA010578

M. Le Huy, C. Amory-Mazaudier, Planetary magnetic signature of the storm wind disturbance dynamo currents: $D_{\text {dyn }}$. J. Geophys. Res. 113, A02312 (2008). doi:10.1029/2007JA012686

J.-L. Le Mouël, V. Kossobokov, V. Courtillot, On long-term variations of simple geomagnetic indices and slow changes in magnetospheric currents: the emergence of anthropogenic global warming after 1990 ? Earth Planet. Sci. Lett. 232, 273-286 (2005)

J.-L. Le Mouël, P. Shebalin, A. Chulliat, The field of the equatorial electrojet from CHAMP data. Ann. Geophys. 24, 515-527 (2006)

P. Le Sager, T.S. Huang, Ionospheric currents and field-aligned currents generated by dynamo action in an asymmetric Earth magnetic field. J. Geophys. Res. 107, 1025 (2002a). doi:10.1029/2001JA000211

P. Le Sager, T.S. Huang, Longitudinal dependence of the daily geomagnetic variation during quiet time. J. Geophys. Res. 107, 1397 (2002b). doi:10.1029/2002JA009287

J.T. Lin, C.H. Lin, L.C. Chang, H.H. Huang, J.Y. Liu, A.B. Chen, C.H. Chen, C.H. Liu, Observational evidence of ionospheric migrating tide modification during the 2009 stratospheric sudden warming. Geophys. Res. Lett. 39, L02101 (2012). doi:10.1029/2011GL050248

R.S. Lindzen, On the theory of the diurnal tide. Mon. Weather Rev. 94, 295-301 (1966)

R.S. Lindzen, S. Chapman, Atmospheric tides. Space Sci. Rev. 10, 3-188 (1969)

H.-L. Liu, A.D. Richmond, Attribution of ionospheric vertical plasma drift perturbations to large-scale waves and the dependence on solar activity. J. Geophys. Res. Space Phys. 118, 2452-2465 (2013). doi:10.1002/jgra.50265

H.-L. Liu, R.G. Roble, A study of a self-generated stratospheric sudden warming and its mesospheric-lower thermospheric impacts using the coupled TIME-GCM/CCM3. J. Geophys. Res. 107(D23), 4695 (2002). doi:10.1029/2001JD001533

H.-L. Liu, W. Wang, A.D. Richmond, R.G. Roble, Ionospheric variability due to planetary waves and tides for solar minimum conditions. J. Geophys. Res. 115, A00G01 (2010). doi:10.1029/2009JA015188

H.-L. Liu, V.A. Yudin, R.G. Roble, Day-to-day ionospheric variability due to lower atmosphere perturbations. Geophys. Res. Lett. 40, 665-670 (2013). doi:10.1002/grl.50125 
J.J. Love, J.L. Gannon, Revised Dst and the epicycles of magnetic disturbance: 1958-2007. Ann. Geophys. 27, 3101-3131 (2009)

J.J. Love, E.J. Rigler, The magnetic tides of Honolulu. Geophys. J. Int. 197, 1335-1353 (2014). doi:10.1093/ gji/ggu090

H. Lühr, C. Manoj, The complete spectrum of the equatorial electrojet related to solar tides: CHAMP observations. Ann. Geophys. 31, 1315-1331 (2013)

H. Lühr, S. Maus, M. Rother, Noon-time equatorial electrojet: Its spatial features as determined by the CHAMP satellite. J. Geophys. Res. 109, A01306 (2004). doi:10.1029/2002JA009656

H. Lühr, M. Rother, K. Häusler, P. Alken, S. Maus, The influence of nonmigrating tides on the longitudinal variation of the equatorial electrojet. J. Geophys. Res. 113, A08313 (2008). doi:10.1029/2008JA013064

H. Lühr, G. Kervalishvili, I. Michaelis, J. Rauberg, P. Ritter, J. Park, J.M.G. Merayo, P. Brauer, The interhemispheric and F region dynamo currents revisited with the swarm constellation. Geophys. Res. Lett. 42, 3069-3075 (2015). doi:10.1002/2015GL063662

H. Lühr, C. Xiong, N. Olsen, G. Le, Near-earth magnetic field effects of large-scale magnetospheric currents. Space Sci. Rev. (2016). doi:10.1007/s11214-016-0267-y

J.W. MacDougall, The equatorial ionospheric anomaly and the equatorial electrojet. Radio Sci. 4, 805-810 (1969)

J.W. MacDougall, Equatorial electrojet and Sq current system, part I. J. Geomagn. Geoelectr. 31, 341-357 (1979a)

J.W. MacDougall, Equatorial electrojet and Sq current system, part II. J. Geomagn. Geoelectr. 31, 359-372 (1979b)

S. Macmillan, A. Droujinina, Long-term trends in geomagnetic daily variation. Earth Planets Space 59, 391395 (2007)

H. Maeda, Horizontal wind systems in the ionospheric E region deduced from the dynamo theory of the geomagnetic Sq variation, I: non-rotating earth. J. Geomagn. Geoelectr. 7, 121-132 (1955)

H. Maeda, Horizontal wind systems in the ionospheric E region deduced from the dynamo theory of the geomagnetic Sq variation, III. J. Geomagn. Geoelectr. 9, 86-93 (1957)

H. Maeda, Field-aligned current induced by asymmetric dynamo action in the ionosphere. J. Atmos. Terr. Phys. 36, 1395-1401 (1974)

K.-I. Maeda, Conductivity and drifts in the ionosphere. J. Atmos. Terr. Phys. 39, 1041-1053 (1977)

H. Maeda, M. Fujiwara, Lunar ionospheric winds deduced from the dynamo theory of geomagnetic variations. J. Atmos. Terr. Phys. 29, 917-936 (1967)

S.R.C. Malin, Separation of lunar daily geomagnetic variations into parts of ionospheric and oceanic origin. Geophys. J. R. Astron. Soc. 21, 447-455 (1970)

S.R.C. Malin, Worldwide distribution of geomagnetic tides. Philos. Trans. R. Soc. Lond. A 274, 551-594 (1973)

S.R.C. Malin, S. Chapman, The determination of lunar daily geophysical variations by the Chapman-Miller Method. Geophys. J. R. Astron. Soc. 19, 15-35 (1970)

S.R.C. Malin, J.C. Gupta, The Sg current system during the international geophysical year. Geophys. J. R. Astron. Soc. 49, 515-529 (1977)

S.R.C. Malin, D.E. Winch, Annual variation of the geomagnetic field. Geophys. J. Int. 124, 170-174 (1996)

S.R.C. Malin, A. Cecere, A. Palumbo, The sunspot cycle influence on lunar and solar daily geomagnetic variations. Geophys. J. R. Astron. Soc. 41, 115-126 (1975)

R.J. Mann, D.M. Schlapp, The equatorial electrojet and the day-to-day variability of Sq. J. Atmos. Terr. Phys. 30, 1761-1776 (1988)

C. Manoj, H. Lühr, S. Maus, N. Nagarajan, Evidence for short spatial correlation lengths of the noontime equatorial electrojet inferred from a comparison of satellite and ground magnetic data. J. Geophys. Res. 111, A11312 (2006). doi:10.1029/2006JA011855

R.T. Marriott, A.D. Richmond, S.V. Venkateswaran, The quiet-time equatorial electrojet and counterelectrojet. J. Atmos. Sol.-Terr. Phys. 31, 311-340 (1979)

N. Maruyama, S. Sazykin, R.W. Spiro, D. Anderson, A. Anghel, R.A. Wold, F.R. Toffoletto, T.J. FullerRowell, M.V. Codrescu, A.D. Richmond, G.H. Millward, Modeling storm-time electrodynamics of the low-latitude ionosphere-thermosphere system: can long lasting disturbance electric fields be accounted for? J. Atmos. Sol.-Terr. Phys. 69, 1182-1199 (2007)

R.G. Mason, The equatorial electrojet in the central Pacific. Mar. Phys. Lab. Scr., Inst. Ocean. Rep. 63, 13 (1963)

S. Matsushita, Effects of solar eclipse on the equatorial geomagnetic field. Ann. Geophys. 22, 471-477 (1966)

S. Matsushita, Solar quiet and lunar daily variation fields, in Physics of Geomagnetic Phenomena, ed. by S. Matsushita, W.H. Campbell (Academic Press, New York, 1967), pp. 302-424

S. Matsushita, Sq and L current systems in the ionosphere. Geophys. J. R. Astron. Soc. 15, 109 (1968) 
S. Matsushita, H. Maeda, On the geomagnetic solar quiet daily variation field during the IGY. J. Geophys. Res. 70, 2535-2558 (1965a)

S. Matsushita, H. Maeda, On the geomagnetic lunar daily variation field. J. Geophys. Res. 70(11), 2559-2578 (1965b). doi:10.1029/JZ070i011p02559

S. Matsushita, W.-Y. Xu, Equivalent ionospheric current systems representing solar daily variations of the polar geomagnetic field. J. Geophys. Res. 87, 8241-8254 (1982)

S. Matsushita, W.-Y. Xu, Seasonal variations of L equivalent current systems. J. Geophys. Res. 89, 285-294 (1984)

J. Matzka, N. Olsen, C. Fox Maule, L. Pedersen, A.M. Berarducci, S. Macmillan, Geomagnetic observations on Tristan da Cuhna, South Atlantic Ocean. Ann. Geophys. 52, 97-105 (2009)

S. Maus, A. Kuvshinov, Ocean tidal signals in observatory and satellite magnetic measurements. Geophys. Res. Lett. 31, L15313 (2004). doi:10.1029/2004GL020090

S. Maus, C. Manoj, J. Rauberg, I. Miehaelis, H. Lühr, NOAA/NGDC candidate models for the 11th generation international geomagnetic reference field and the concurrent release of the 6th generation Pomme magnetic model. Earth Planets Space 62, 729-735 (2010)

A. Maute, A.D. Richmond, F-region dynamo simulations at low and mid-latitude. Space Sci. Rev. (2016). doi:10.1007/s11214-016-0262-3

A. Maute, M.E. Hagan, A.D. Richmond, R.G. Roble, TIME-GCM study of the ionospheric equatorial vertical drift changes during the 2006 stratospheric sudden warming. J. Geophys. Res. Space Phys. 119, $1287-$ 1305 (2014). doi:10.1002/2013JA019490

P.N. Mayaud, Analyse morphologique de la variabilité jour à jour de la variation "régulière" $\mathrm{Sr}$ du champ magnétique terrestre, 1, le système de courants $\mathrm{Cm}$ (régions non polaires). Ann. Geophys. 21, 515 (1965)

P.N. Mayaud, The equatorial counter-electrojet—a review of its geomagnetic aspects. J. Atmos. Terr. Phys. 39, 1055-1070 (1977)

P.N. Mayaud, Derivation, Meaning, and Use of Geomagnetic Indices. Washington DC American Geophysical Union Geophysical Monograph Series, vol. 22 (1980)

N.C. Maynard, Measurements of ionospheric currents off the coast of Peru. J. Geophys. Res. 72(7), 18631875 (1967). doi:10.1029/JZ072i007p01863

A.J. McDonald, R.E. Hibbins, M.J. Jarvis, Properties of the quasi 16 day wave derived from EOS MLS observations. J. Geophys. Res. 116, D06112 (2011). doi:10.1029/2010JD014719

J.D. McKnight, Lunar daily geomagnetic variations in New Zealand. Geophys. J. Int. 122, 889-898 (1995)

A.G. McNish, Magnetic effects associated with bright solar eruptions and radio fade-outs. Nature 139, 244 (1937a)

A.G. McNish, Terrestrial magnetic and ionospheric effects associated with bright chromospheric eruptions. Terr. Magn. Atmos. Electr. 42, 109-122 (1937b)

G.D. Mead, Deformation of the geomagnetic field by the solar wind. J. Geophys. Res. 69(7), 1181-1195 (1964). doi:10.1029/JZ069i007p01181

S. Miyahara, M. Ooishi, Variation of Sq induced by atmospheric tides simulated by a middle atmosphere general circulation model. J. Geomagn. Geoelectr. 49, 77-87 (1997)

M.A. Momani, B. Yatim, M.A. Mohd Ali, Ionospheric and geomagnetic response to the total solar eclipse on 1 August 2008 over Northern Hemisphere. J. Geophys. Res. 115, A08321 (2010). doi:10.1029/2009JA014999

Y. Moudden, J.M. Forbes, Quasi-two-day wave structure, interannual variability, and tidal interactions during the 2002-2011 decade. J. Geophys. Res., Atmos. 119, 2241-2260 (2014). doi:10.1002/2013JD020563

P. Muralikrishna, V.H. Kulkarni, Modeling the meteoric dust effect on the equatorial electrojet. Adv. Space Res. 42, 164-170 (2008). doi:10.1016/asr.2007.11.019

K. Mursula, I. Usoskin, O. Yakovchouk, Does sunspot number calibration by the "magnetic needle" make sense? J. Atmos. Sol.-Terr. Phys. 71, 1717-1726 (2009)

T. Nagata, Characteristics of the solar flare effect (Sqa) on geomagnetic field at Huancayo (Peru) and at Kakioka (Japan). Terr. Magn. Atmos. Electr. 57, 1-14 (1952)

T. Nagata, Solar flare effect on the geomagnetic field. J. Geomagn. Geoelectr. 18, 197-219 (1966)

T. Nagata, S. Kokubun, A particular geomagnetic daily variation $\left(\mathrm{Sq}^{p}\right)$ in the polar regions on geomagnetically quiet days. Nature 195, 555-557 (1962)

T. Nagata, Y. Nakata, T. Rikitake, I. Yokoyama, Effect of the solar eclipse on the lower parts of the ionosphere and the geomagnetic field. Rep. Ionos. Res. Jpn. 9, 121-135 (1955)

K.N. Nair, R.G. Rastogi, V. Sarabhai, Daily variation of the geomagnetic field at the dip equator. Nature 226, 740-741 (1970)

H. Nevanlinna, L. Häkkinen, Geomagnetic effec of the total solar eclipse on July 22, 1990. J. Geomagn. Geoelectr. 43, 319-321 (1991) 
P.T. Newell, J.W. Gjerloev, SuperMAG-based partial ring current indices. J. Geophys. Res. 117, A05215 (2012). doi:10.1029/2012JA017586

A. Nishida, Coherence of geomagnetic DP 2 fluctuations with interplanetary magnetic variations. J. Geophys. Res. 73(17), 5549-5559 (1968). doi:10.1029/JA073i017p05549

J. Oberheide, J.M. Forbes, K. Häusler, Q. Wu, S.L. Bruinsma, Tropospheric tides from 80 to $400 \mathrm{~km}$ : propagation, interannual variability, and solar cycle effects. J. Geophys. Res. 114, D00I05 (2009). doi:10.1029/2009JD012388

P.O. Ogbuehi, A. Onwumechilli, Recent measurements of the magnetic field of the equatorial electrojet in Nigeria. J. Geophys. Res. 68(9), 2421-2424 (1963)

P.O. Ogbuehi, A. Onwumechilli, S.O. Ifedili, The equatorial electrojet and the world-wide Sq currents. J. Atmos. Terr. Phys. 29, 149-160 (1967)

M. Ohshio, N. Fuskushima, T. Nagata, Solar flare effect on geomagneticvariation. Rep. Ionos. Space Res. Jpn. 17, 77-114 (1963)

F.N. Okeke, D.N. Obiora, Application of solar quiet day (Sq) current in determining mantle electrical-depth conductivity structure - a review. J. Afr. Earth Sci. 114, 54-62 (2016)

N. Olsen, The solar cycle variability of lunar and solar daily geomagnetic variations. Ann. Geophys. 11, 254-262 (1993)

N. Olsen, Magnetospheric contributions to geomagnetic daily variations. Ann. Geophys. 14, 538-544 (1996)

N. Olsen, Geomagnetic tides and related phenomena, in Tidal Phenomena (Springer, Berlin, 1997a), pp. 261-274

N. Olsen, Ionospheric F region currents at middle and low latitudes estimated from Magsat data. J. Geophys. Res. 102(A3), 4563-4576 (1997b)

N. Olsen, C. Stolle, Satellite geomagnetism. Annu. Rev. Earth Planet. Sci. 40, 441-465 (2012)

W.P. Olson, Contribution of nonionospheric currents to the quiet daily magnetic variations at the earth's surface. J. Geophys. Res. 75, 7244-7249 (1970)

C.A. Onwumechili, A study of the equatorial electrojet, I: an experimental study. J. Atmos. Terr. Phys. 13, 222-234 (1959)

A. Onwumechili, On the existence of days with extraordinary geomagnetic lunar tide. J. Atmos. Terr. Phys. 26, 729-748 (1964). doi:10.1016/0021-9169(64)90161-8

C.A. Onwumechili, Study of the return current of the equatorial electrojet. J. Geomagn. Geoelectr. 44, 1-42 (1992)

C.A. Onwumechili, The Equatorial Electrojet (Gordon and Breach, Newark, 1998)

C.A. Onwumechili, C.E. Agu, Longitudinal variation of equatorial electrojet parameters derived from POGO satellite observations. Planet. Space Sci. 29, 627-634 (1981)

A.L. Orozco, L.M. Barreto, Magnetic effects during the solar eclipse of July 11, 1991. Geofís. Int. 32, 3-13 (1993)

D.G. Osborne, Daily variability in strength of equatorial electrojet. J. Geophys. Res. 68, 2435-2439 (1963)

D.G. Osborne, Correlations between quiet-day magnetic ranges. J. Atmos. Terr. Phys. 28, 45-51 (1966)

D.G. Osborne, Some feature correlations in magnetic variation. J. Atmos. Terr. Phys. 30, 1479-1486 (1968)

D. Pancheva, P. Mukhtarov, Stratospheric warmings: the atmosphere-ionosphere coupling paradigm. J. Atmos. Sol.-Terr. Phys. 73, 1697-1702 (2011)

D.V. Pancheva, P.J. Mukhtarov, M.G. Shepherd, N.J. Mitchell, D.C. Fritts, D.M. Riggin, S.J. Franke, P.P. Batista, M.A. Abdu, I.S. Batista, B.R. Clemesha, T. Kikuchi, Two-day wave coupling of the low-latitude atmosphere-ionosphere system. J. Geophys. Res. 111, A07313 (2006). doi:10.1029/2005JA011562

D.V. Pancheva, P.J. Mukhtarov, N.J. Mitchell, D.C. Fritts, D.M. Riggin, H. Takahashi, P.P. Batista, B.R. Clemesha, S. Gurubaran, G. Ramkumar, Planetary wave coupling (5-6-day waves) in the low-latitude atmosphere-ionosphere system. J. Atmos. Sol.-Terr. Phys. 70, 101-122 (2008)

J. Park, H. Lühr, K.W. Min, Climatology of the inter-hemispheric field-aligned current system in the equatorial ionosphere as observed by CHAMP. Ann. Geophys. 29, 573-582 (2011). doi:10.5194/ angeo-29-573-2011

J. Park, H. Lühr, M. Kunze, B.G. Fejer, K.W. Min, Effect of sudden stratospheric warming on lunar tidal modulation of the equatorial electrojet. J. Geophys. Res. 117, A03306 (2012). doi:10.1029/2011JA017351

W.D. Parkinson, An analysis of the geomagnetic diurnal variations during the international geophysical year. Bur. Miner. Resour., Geol. Geophys., Bull. (Aust.) 173 (1977)

A.R. Patil, R. Rajaram, A test for the stationariness of the Sq current system. J. Geophys. Res. 106(A9), 18589-18596 (2001). doi:10.1029/2000JA000399

N.M. Pedatella, Observations and simulations of the ionospheric lunar tide: seasonal variability. J. Geophys. Res. Space Phys. 119, 5800-5806 (2014). doi:10.1002/2014JA020189

N.M. Pedatella, H.-L. Liu, The influence of atmospheric tide and planetary wave variability during sudden stratosphere warmings on the low latitude ionosphere. J. Geophys. Res. Space Phys. 118, 5333-5347 (2013). doi:10.1002/jgra.50492 
N.M. Pedatella, J.M. Forbes, A.D. Richmond, Seasonal and longitudinal variations of the solar quiet (Sq) current system during solar minimum determined by CHAMP satellite magnetic field observations. J. Geophys. Res. 116, A04317 (2011). doi:10.1029/2010JA016289

N.M. Pedatella, H.-L. Liu, A.D. Richmond, Atmospheric semidiurnal lunar tide climatology simulated by the whole atmosphere community climate model. J. Geophys. Res. 117, A06327 (2012a). doi:10.1029/ 2012JA017792

N.M. Pedatella, H.-L. Liu, A.D. Richmond, A. Maute, T.-W. Fang, Simulations of solar and lunar tidal variability in the mesosphere and lower thermosphere during sudden stratosphere warmings and their influence on the low-latitude ionosphere. J. Geophys. Res. 117, A08326 (2012b). doi:10.1029/ 2012JA017858

N.M. Pedatella, T. Fuller-Rowell, H. Wang, H. Jin, Y. Miyoshi, H. Fujiwara, H. Shinagawa, H.-L. Liu, F. Sassi, H. Schmidt, V. Matthias, L. Goncharenko, The neutral dynamics during the 2009 sudden stratosphere warming simulated by different whole atmosphere models. J. Geophys. Res. Space Phys. 119, 1306-1324 (2014b). doi:10.1002/2013JA019421

N.M. Pedatella, H.-L. Liu, F. Sassi, J. Lei, J.L. Chau, X. Zhang, Ionosphere variability during the 2009 SSW: influence of the lunar semidiurnal tide and mechanisms producing electron density variability. J. Geophys. Res. Space Phys. 119, 3828-3843 (2014a). doi:10.1002/2014JA019849

C.L. Pekeris, Atmospheric oscillations. Proc. R. Soc. A 158, 650-671 (1937)

C. Peymirat, A.D. Richmond, A.T. Kobea, Electrodynamic coupling of high and low latitudes: simulations of shielding/overshielding effects. J. Geophys. Res. 105(A10), 22991-23003 (2000). doi:10.1029/ 2000JA000057

R.F. Pfaff Jr., M.H. Acuna, P.A. Marionni, N.B. Trivedi, DC polarization electric field, current density, and plasma density measurements in the daytime equatorial electrojet. Geophys. Res. Lett. 24, 1667-1670 (1997)

H. Pham Thi Thu, C. Amory-Mazaudier, M. Le Huy, Sq field characteristics at Phu Thuy, Vietnam, during solar cycle 23: comparisons with Sq field in other longitude sectors. Ann. Geophys. 29, 1-17 (2011)

A.T. Price, The noncyclic variation during quiet days. J. Geophys. Res. 68, 6383-6389 (1963)

A.T. Price, G.A. Wilkins, New methods for the analysis of geomagnetic fields and their application to the Sq field of 1932-3. Philos. Trans. R. Soc. 256, 31-98 (1963)

L. Qian, A.G. Burns, B.A. Emery, B. Foster, G. Lu, A. Maute, A.D. Richmond, R.G. Roble, S.C. Solomon, W. Wang, The NCAR TIE-GCM, in Modeling the Ionosphere-Thermosphere System, ed. by J. Huba, R. Schunk, G. Khazanov (Wiley, Chichester, 2014). doi:10.1002/9781118704417.ch7

A.B. Rabiu, N. Nagarajan, Inter-relationships between the thickness, width and intensity of the equatorial electrojet in Indian sector. Bull. Astron. Soc. India 35, 645-654 (2007)

A.B. Rabiu, N. Nagarajan, F.N. Okeke, E.A. Ariyibi, G.M. Olayanju, E.O. Joshua, V.U. Chukwuma, A study of day-to-day variability in geomagnetic field variations at the electrojet zone of Addis Ababa, East Africa. Afr. J. Sci. Technol. 8, 54-63 (2007)

A.B. Rabiu, C.A. Onwumechili, N. Nagarajan, K. Yumoto, Characteristics of equatorial electrojet over India determined from a thick current shell model. J. Atmos. Sol.-Terr. Phys. 92, 105-115 (2013)

R. Raghavarao, B.G. Anandarao, Vertical winds as a plausible cause for equatorial counter electrojet. Geophys. Res. Lett. 7, 357-360 (1980)

R. Raghavarao, P. Sharma, M.R. Sivaraman, Correlation of ionization anomaly with the intensity of the electrojet. Space Res. XVIII, 277-280 (1978)

M. Rajaram, Determination of the latitude of Sq focus and its relation to the electrojet variations. J. Atmos. Terr. Phys. 45, 573-578 (1983)

P.V.S. Rama Rao, S. Gopi Krishna, K. Niranjan, D.S.V.V.D. Prasad, Temporal and spatial variations in TEC using simultaneous measurements from the Indian GPS network of receivers during the low solar activity period of 2004-2005. Ann. Geophys. 24, 3279-3292 (2006)

T.K. Ramkumar, S. Gurubaran, R. Rajaram, Mesospheric planetary wave signatures in the equatorial electrojet. J. Geophys. Res. 114, A03309 (2009). doi:10.1029/2007JA012935

T.K. Ramkumara, Y. Bhavanikumar, D. Narayana Rao, S. Gurubaran, A. Narendra Babu, A.K. Ghosh, R. Rajaram, Observational evidences on the influences of tropical lower atmospheric $\sim 20$ day oscillation on the ionospheric equatorial electrojet. J. Atmos. Sol.-Terr. Phys. 68, 523-538 (2006)

G.K. Rangarajan, Quasi-bidaily variation of the geomagnetic field in the Indian equatorial zone. J. Geomagn. Geoelectr. 46, 373-380 (1994)

R.G. Rastogi, Longitudinal variation in the equatorial electrojet. J. Atmos. Terr. Phys. 24, 1031-1040 (1962)

R.G. Rastogi, Lunar effects in the counter electrojet near the magnetic equator. J. Atmos. Terr. Phys. 36, 167-170 (1974)

R.G. Rastogi, Geomagnetic storms and electric fields in the equatorial ionosphere. Nature 268, 422-424 (1977) 
R.G. Rastogi, Solar eclipse effects on geomagnetism. Proc. Indian Natl. Sci. Acad., A, Phys. Sci. 48, 464-472 (1982)

R.G. Rastogi, The equatorial electrojet: magnetic and ionsopheric effects, in Geomagnetism, vol. 3 (1989), pp. 461-525

R.G. Rastogi, Disintegration of the ionospheric Sq loop system during winter solstices along $75^{\circ} \mathrm{E}$ longitude. Ann. Geophys. 11, 40-46 (1993)

R.G. Rastogi, Ionospheric current system associated with the equatorial counterelectrojet. J. Geophys. Res. 99, 13209-13217 (1994)

R.G. Rastogi, Midday reversal of equatorial ionospheric electric field. Ann. Geophys. 15, 1309-1315 (1997)

R.G. Rastogi, Morphological aspects of a new type of counter electrojet event. Ann. Geophys. 17, 210-219 (1999)

R.G. Rastogi, Effect of solar disturbances on the geomagnetic H, Y and Z fields in American equatorial electrojet stations I solar flare effects. J. Indian Geophys. Union 7, 43-51 (2003)

R.G. Rastogi, Equatorial electrojet at a close pair of stations. Curr. Sci. (India) 91, 272-273 (2006)

R.G. Rastogi, H. Chandra, Equatorial electrojet in the African sector. Indian J. Radio Space Phys. 44, 187-198 (2015)

R.G. Rastogi, K.N. Iyer, Quiet day variation of geomagnetic H-field at low latitudes. J. Geomagn. Geoelectr. 28, 461-479 (1976)

R.G. Rastogi, J.A. Klobuchar, Ionospheric electron content within the equatorial F2 layer anomaly belt. J. Geophys. Res. 95, 19045-19052 (1990)

R.G. Rastogi, V.L. Patel, Effect of interplanetary magnetic field on ionosphere over the magnetic equator. Proc. Indian Acad. Sci. A 82, 121-141 (1975)

R.G. Rastogi, A. Patil, Complex structure of equatorial electrojet current. Curr. Sci. 55, 433-436 (1986)

R.G. Rastogi, G. Rajaram, Electrojet effects on the equatorial F region during magnetically quiet and disturbed days. Indian J. Pure Appl. Phys. 9, 531-536 (1971)

R.G. Rastogi, N.B. Trivedi, Luni-solar tides in $\mathrm{H}$ at stations within the equatorial electrojet. Planet. Space Sci. 18, 367-377 (1970)

R.G. Rastogi, S. Alex, A. Patil, Seasonal variations of geomagnetic D, H and Z fields at low latitudes. J. Geomagn. Geoelectr. 46, 115-126 (1994)

R.G. Rastogi, B.M. Pathan, D.R.K. Rao, T.S. Sastry, J.H. Sastri, Solar flare effects on the geomagnetic elements during normal and counter electrojet periods. Earth Planets Space 51, 947-957 (1999)

R.G. Rastogi, T. Kitamura, K. Kitamura, Geomagnetic field variations at the equatorial electrojet station in Sri Lanka, Peredinia. Ann. Geophys. 22, 2729-2739 (2004)

R.G. Rastogi, H. Chandra, M.E. James, K. Kitamura, K. Yumoto, Characteristics of the equatorial electrojet current in the central region of South America. Earth Planets Space 60, 623-632 (2008)

R.G. Rastogi, H. Chandra, K. Yumoto, Equatorial electrojet in east Brazil longitudes. J. Earth Syst. Sci. 119(4), 497-505 (2010)

C.A. Reddy, The equatorial electrojet. Pure Appl. Geophys. 131, 485-508 (1989)

A.D. Richmond, Equatorial electrojet, I: development of a model including winds and instabilities. J. Atmos. Terr. Phys. 35, 1083-1103 (1973)

A.D. Richmond, Ionospheric wind dynamo theory: a review. J. Geomagn. Geoelectr. 31 (1979)

A.D. Richmond, Modeling the ionosphere wind dynamo: a review. quiet daily geomagnetic fields. Pure Appl. Geophys. 131, 413-435 (1989)

A.D. Richmond, Ionospheric electrodynamics, in Handbook of atmospheric electrodynamics, vol. 2, ed. by H. Volland (CRC Press, Boca Raton, 1995a), pp. 249-290

A.D. Richmond, The ionospheric wind dynamo: effects of its coupling with different atmospheric regions, in The Upper Mesosphere and Lower Thermosphere: A Review of Experiment and Theory, ed. by R.M. Johnson, T.L. Killeen (Am. Geophys. Union, Washington, 1995b), pp. 49-65

A.D. Richmond, A. Maute, Ionospheric electrodynamics modeling, in Modeling the IonosphereThermosphere System, ed. by J. Huba, R. Schunk, G. Khazanov (Wiley, Chichester, 2014). doi:10.1002/ 9781118704417.ch6

A.D. Richmond, R.G. Roble, Electrodynamic effects of thermospheric winds from the NCAR thermospheric general circulation model. J. Geophys. Res. 92, 12365-12376 (1987)

A.D. Richmond, S.V. Venkateswaran, Geomagnetic crochets and associated ionospheric current systems. Radio Sci. 6(2), 139-164 (1971). doi:10.1029/RS006i002p00139

A.D. Richmond, S. Matsushita, J.D. Tarpley, On the production mechanism of electric currents and fields in the ionosphere. J. Geophys. Res. 81, 547-555 (1976)

A.D. Richmond, E.C. Ridley, R.G. Roble, A thermosphere/ionosphere general circulation model with coupled electrodynamics. Geophys. Res. Lett. 19, 601-604 (1992)

D.M. Riggin, H.-L. Liu, R.S. Lieberman, R.G. Roble, J.M. Russell III, C.J. Mertens, M.G. Mlynczak, D. Pancheva, S.J. Franke, Y. Murayama, A.H. Manson, C.E. Meek, R.A. Vincent, Observations of the 5-day wave in the mesosphere and lower thermosphere. J. Atmos. Sol.-Terr. Phys. 68, 323-339 (2006) 
A. Rigoti, F.H. Chamalaun, N.B. Trivedi, A.L. Padilha, Characteristics of the equatorial electrojet determined from an array of magnetometers in N-NE Brazil. Earth Planets Space 51, 115-128 (1999)

K.F. Riley, M.P. Hobson, S.J. Bence, Mathematical Methods for Physics and Engineering: A Comprehensive Guide, 2nd edn. (Cambridge Univ. Press, Cambridge, 2002)

R.G. Roble, E.C. Ridley, An auroral model for the NCAR thermospheric general circulation model (TGCM). Ann. Geophys. 5A(6), 369-382 (1987)

M.N. Ross, R.L. Walterscheid, Changes in the solar forced tides caused by stratospheric ozone depletion. Geophys. Res. Lett. 18, 420-423 (1991)

C.M. Rush, A.D. Richmond, The relationship between the structure of the equatorial anomaly and the strength of the equatorial electrojet. J. Atmos. Terr. Phys. 35, 1171-1180 (1973)

C.T. Russell, R.L. McPherron, Semiannual variation of geomagnetic activity. J. Geophys. Res. 78(1), 92-108 (1973). 1973. doi:10.1029/JA078i001p00092

T.J. Sabaka, N. Olsen, R.A. Langel, A comprehensive model of the quiet-time, near-Earth magnetic field: phase 3. Geophys. J. Int. 151, 32-68 (2002). doi:10.1046/j.1365-246X.2002.01774.x

T.J. Sabaka, N. Olsen, R.H. Tyler, A. Kuvshinov, CM5, a pre-Swarm comprehensive geomagnetic field model derived from over $12 \mathrm{yr}$ of CHAMP, Ørsted, SAC-C and observatory data. Geophys. J. Int. 200, 15961626 (2015). doi:10.1093/gji/ggu493

T.J. Sabaka, R.H. Tyler, N. Olsen, Extracting ocean-generated tidal magnetic signals from Swarm data through satellite gradiometry. Geophys. Res. Lett. 43, 3237-3245 (2016)

M.L. Salby, Survey of planetary-scale traveling waves: The state of theory and observations. Rev. Geophys. 22, 209-236 (1984)

J.H. Sastri, Night time geomagnetic effects of solar flares. Ann. Geophys. 31, 389-393 (1975)

N.S. Sastri, B.R. Arora, Lunar modulation of the occurrence frequency of the afternoon counter-electrojet events at Trivandrum. Planet. Space Sci. 10, 1091-1094 (1981)

J.H. Sastri, B.S. Murthy, Geomagnetic effects in the dark hemisphere associated with solar flares. J. Geomagn. Geoelectr. 27, 67-73 (1975)

N.S. Sastri, D.R.K. Rao, Separation of lunar daily geomagnetic variations into parts of oceanic and ionospheric origin in the Indian region. Geophys. J. R. Astron. Soc. 23, 269-272 (1971)

J.P. Schieldge, S.V. Venkateswaran, A.D. Richmond, The ionospheric dynamo and equatorial magnetic variations. J. Atmos. Terr. Phys. 35, 1045-1061 (1973)

D.M. Schlapp, World-wide morphology of day-to-day variability of Sq. J. Atmos. Terr. Phys. 30, 1761-1776 (1968)

D.M. Schlapp, R. Sellek, E.C. Butcher, Studies of worldwide secular trends in the solar daily geomagnetic variation. Geophys. J. Int. 100, 469-475 (1990)

U. Schmucker, An introduction to induction anomalies. J. Geomagn. Geoelectr. 22, 9-33 (1970)

A. Schuster, The diurnal variation of terrestrial magnetism. Philos. Trans. R. Soc. Lond. Ser. A 180, 467-518 (1889)

A. Schuster, The diurnal variation of terrestrial magnetism. Philos. Trans. R. Soc. Lond. Ser. A 208, 163-204 (1908)

R. Sellek, Secular trends in daily geomagnetic variations. J. Atmos. Sol.-Terr. Phys. 42, 689-695 (1980)

C. Senior, M. Blanc, On the control of magnetospheric convection by the spatial distribution of ionospheric conductivities. J. Geophys. Res. 89(A1), 261-284 (1984). 1984. doi:10.1029/JA089iA01p00261

A. Shinbori, Y. Koyama, M. Nose, T. Hori, Y. Otsuka, A. Yatagai, Long-term variation in the upper atmosphere as seen in the geomagnetic solar quiet daily variation. Earth Planets Space 66, 155 (2014). doi:10.1186/s40623-014-0155-1

M. Shiraki, Variation of focus latitude and intensity of overhead current system of Sq with the solar activity. Mem. Kakioka Magn. Obs. 15, 107-126 (1973)

M. Shiraki, Effect of the Oceanic Dynamo on the Lunar Daily Geomagnetic Variation at Kakioka, Memambetsu and Kanoya, Japan, 1958-1973. Mem. Kakioka Magn. Obs. 17, 51-58 (1978)

M. Shiraki, Seasonal dependence of lunar daily geomagnetic variations in different regions of the world. J. Geomagn. Geoelectr. 33, 467-501 (1981)

T.A. Siddiqui, H. Lühr, C. Stolle, J. Park, Relation between stratospheric sudden warming and the lunar effect on the equatorial electrojet based on Huancayo recordings. Ann. Geophys. 33, 235-243 (2015a). doi:10.5194/angeo-33-235-2015

T.A. Siddiqui, C. Stolle, H. Lühr, J. Matzka, On the relationship between weakening of the northern polar vortex and the lunar tidal amplification in the equatorial electrojet. J. Geophys. Res., Atmos. 120, 1000610019 (2015b). doi:10.1002/2015JA021683

S.F. Singer, E. Maple, W.A. Bowen Jr., Evidence for ionosphere currents from rocket experiments near the geomagnetic equator. J. Geophys. Res. 56, 265-281 (1951)

V. Sreeja, C.V. Devasia, S. Ravindran, T.K. Pant, Observational evidence for the plausible linkage of equatorial electrojet (EEJ) electric field variations with the post sunset F-region electrodynamics. Ann. Geophys. 27, 4229-4238 (2009) 
S. Sridharan, S. Sathishkumar, S. Gurubaran, Variabilities of mesospheric tides and equatorial electrojet strength during major stratospheric warming events. Ann. Geophys. 27, 4125-4130 (2009)

P. Stauning, Determination of the quiet daily geomagnetic variations for polar regions. J. Atmos. Sol.-Terr. Phys. 73, 2314-2330 (2011)

R.J. Stening, An assessment of the contributions of various tidal winds to the Sq current system. Planet. Space Sci. 17, 889-908 (1969)

R.J. Stening, Longitude and seasonal variations of the Sq current system. Radio Sci. 6, 133-137 (1971)

R.J. Stening, Field-aligned currents driven by the ionospheric dynamo. J. Atmos. Terr. Phys. 39, 933-937 (1977a)

R.J. Stening, Magnetic variations at other latitudes during reverse equatorial electrojet. J. Atmos. Terr. Phys. 39, 1071-1077 (1977b)

R.J. Stening, Analysis of contributions to ionospheric dynamo currents from e.m.f.'s at different latitudes. Planet. Space Sci. 25, 587-594 (1977c)

R.J. Stening, Electron density profile changes associated with the equatorial electrojet. J. Atmos. Terr. Phys. 39, 157-164 (1977d)

R.J. Stening, Variability of the equatorial electrojet: its relations to the Sq current system and semidiurnal tides. Geophys. Res. Lett. 18, 1979-1982 (1991)

R.J. Stening, What drives the equatorial electrojet? J. Atmos. Terr. Phys. 57, 1117-1128 (1995a)

R.J. Stening, Variations in the strength of the Sq current system. Ann. Geophys. 13, 627-632 (1995b)

R.J. Stening, The shape of the Sq current system. Ann. Geophys. 26, 1767-1775 (2008)

R.J. Stening, Lunar tide in the equatorial electrojet in relation to stratospheric warmings. J. Geophys. Res. 116, A12315 (2011). doi:10.1029/2011JA017047

R.J. Stening, D.E. Winch, Seasonal changes in the global lunar geomagnetic variation. J. Atmos. Terr. Phys. 41, 311-323 (1979)

R.J. Stening, D.E. Winch, The ionospheric Sq current system obtained by spherical harmonic analysis. J. Geophys. Res. Space Phys. 118, 1288-1297 (2013). doi:10.1002/jgra.50194

R.J. Stening, J.C. Gupta, G. Jansen Van Beek, Magnetic observations in Canada during the solar eclipse of March 7, 1970. Nature (Phys. Sci.) 230, 22-23 (1971)

R.J. Stening, C.E. Meek, A.H. Manson, Upper atmosphere wind systems during reverse equatorial electrojet events. Geophys. Res. Lett. 23, 3243-3246 (1996)

R.J. Stening, J.M. Forbes, M.E. Hagan, A.D. Richmond, Experiments with a lunar atmospheric model. J. Geophys. Res. 102, 13465-13471 (1997). doi:10.1029/97JD00778

R. Stening, T. Reztsova, D. Ivers, J. Turner, D. Winch, A critique of methods of determining the position of the focus of the Sq current system. J. Geophys. Res. 110, A04305 (2005a). doi:10.1029/2004JA010784

R. Stening, T. Reztsova, L.H. Minh, Day-to-day changes in the latitudes of the foci of the Sq current system and their relation to equatorial electrojet strength. J. Geophys. Res. 110, A10308 (2005b). doi:10.1029/2005JA011219

R. Stening, T. Reztsova, M. Le Huy, Variation of Sq focus latitudes in the Australian/Pacific region during a quiet sun year. J. Atmos. Sol.-Terr. Phys. 69, 734-740 (2007)

B. Stewart, Hypothetical views regarding the connection between the state of the sun and terrestrial magnetism, in Encyclopedia Brittanica, vol. 16, 9th edn. (1882), pp. 181-184

C. Stolle, H. Lühr, M. Rother, G. Balasis, Magnetic signatures of equatorial spread F as observed by the CHAMP satellite. J. Geophys. Res. 111, A02304 (2006). doi:10.1029/2005JA011184

C. Stolle, C. Manoj, H. Lühr, S. Maus, P. Alken, Estimating the daytime equatorial ionization anomaly strength from electric field proxies. J. Geophys. Res. 113, A09310 (2008). doi:10.1029/2007JA012781

C. Stolle, I. Michaelis, J. Rauberg, The role of high-resolution geomagnetic field models for investigating ionospheric currents at low Earth orbit satellites. Earth Planets Space 68, 110 (2016). doi:10.1186/ s40623-016-0494-1

M. Sugiura, G. Fanselau, Lunar phase numbers $v$ and $v^{\prime}$ for years 1850 to 2050. Goddard Space Flight Center report X-612-66-401 (1966)

M. Sugiura, D.J. Poros, An improved model of equatorial electrojet with a meridional current system. J. Geophys. Res. 74, 4025-4034 (1969)

A. Suzuki, A new analysis of the geomagnetic Sq field. J. Geomagn. Geoelectr. 25, 259-280 (1973)

A. Suzuki, Geomagnetic Sq field at successive universal times. J. Atmos. Terr. Phys. 40, 449-463 (1978)

A. Suzuki, UT and day-to-day variations in equivalent current systems for world geomagnetic variations. J. Geomagn. Geoelectr. 31, 21-46 (1979)

L. Svalgaard, Reconstruction of solar extreme ultraviolet flux 1740-2015. Sol. Phys. (2016, in press)

L. Svalgaard, E.W. Cliver, Calibrating the sunspot number using "the magnetic needle". CAWSES Newsl. 4(1), 6-8 (2007). http://www.leif.org/research/CAWSES\%20-\%20Sunspots.pdf

M. Takeda, Three dimensional ionospheric currents and field aligned currents generated by asymmetrical dynamo action in the ionosphere. J. Atmos. Terr. Phys. 44, 187-193 (1982) 
M. Takeda, Day-to-day variation of equivalent Sq current system during March 11-26, 1970. J. Geomagn. Geoelectr. 36, 215-338 (1984)

M. Takeda, UT variation of internal Sq currents and the oceanic effect during 1980 March 1-18. Geophys. J. R. Astron. Soc. 80, 649-659 (1985)

M. Takeda, Geomagnetic field variation and the equivalent current system generated by an ionospheric dynamo at the solstice. J. Atmos. Terr. Phys. 52, 59-67 (1990)

M. Takeda, Role of Hall conductivity in the ionospheric dynamo. J. Geophys. Res. 96, 9755-9759 (1991)

M. Takeda, Effects of the strength of the geomagnetic main field strength on the dynamo action in the ionosphere. J. Geophys. Res. 101, 7875-7880 (1996)

M. Takeda, Time variation of global geomagnetic Sq field in 1964 and 1980. J. Atmos. Sol.-Terr. Phys. 61, 765-774 (1999)

M. Takeda, Features of global geomagnetic Sq field from 1980 to 1990 . J. Geophys. Res. 107(A9), 1252 (2002a). doi:10.1029/2001JA009210

M. Takeda, The correlation between the variation in ionospheric conductivity and that of the geomagnetic Sq field. J. Atmos. Sol.-Terr. Phys. 64, 1617-1621 (2002b)

M. Takeda, Difference in seasonal and long-term variations in geomagnetic Sq fields between geomagnetic Y and Z components. J. Geophys. Res. Space Phys. 118, 2522-2526 (2013a). doi:10.1002/jgra.50128

M. Takeda, Contribution of wind, conductivity, and geomagnetic main field to the variation in the geomagnetic Sq field. J. Geophys. Res. Space Phys. 118, 4516-4522 (2013b). doi:10.1002/jgra.50386

M. Takeda, Ampère force exerted by geomagnetic Sq currents and thermospheric pressure difference. J. Geophys. Res. Space Phys. 120, 3847-3853 (2015). doi:10.1002/2014JA020952

M. Takeda, T. Araki, Time variation of instantaneous equivalent Sq current system. J. Atmos. Terr. Phys. 46, 911-915 (1984a)

M. Takeda, T. Araki, Ionospheric currents and fields during during the solar eclipse. Planet. Space Sci. 32, 1013-1019 (1984b)

M. Takeda, T. Araki, Electric conductivity of the ionosphere and nocturnal currents. J. Atmos. Terr. Phys. 47, 601-609 (1985)

M. Takeda, H. Maeda, Three-dimensional structure of ionospheric currents, 1: currents caused by diurnal tidal winds. J. Geophys. Res. 85, 6895-6899 (1980a)

M. Takeda, H. Maeda, Equivalent Sq current system at occasions of the equatorial counter electrojet. J. Geomagn. Geoelectr. 32, 297-301 (1980b)

M. Takeda, H. Maeda, Three-dimensional structure of ionospheric currents, 2: currents caused by semidiurnal tidal winds. J. Geophys. Res. 86, 5861-5867 (1981)

M. Takeda, Y. Yamada, Quasi two-day period variation of the geomagnetic field. J. Geomagn. Geoelectr. 41, 469-478 (1989)

M. Takeda, T. Iyemori, A. Saito, Relationship between electric field and currents in the ionosphere and the geomagnetic Sq field. J. Geophys. Res. 108(A5), 1183 (2003). doi:10.1029/2002JA009659

J.D. Tarpley, The ionospheric wind dynamo, II. Planet. Space Sci. 18, 1091-1103 (1970)

J.D. Tarpley, Seasonal movement of the Sq current loci and related effects in the equatorial electrojet. J. Atmos. Terr. Phys. 35, 1063-1071 (1973)

A.T. Tomás, H. Lühr, M. Rother, C. Manoj, N. Olsen, S. Watari, J. Atmos. Sol.-Terr. Phys. 70, 1497-1511 (2008)

J.M. Torta, J.J. Curto, P. Bencze, Behavior of the quiet day ionospheric current system in the European region. J. Geophys. Res. 102(A2), 2483-2494 (1997). doi:10.1029/96JA03463

J.M. Torta, L.R. Gaya-Piqué, J.J. Curto, D. Altadill, An inspection of the long-term behaviour of the range of the daily geomagnetic field variation from comprehensive modelling, vol. 71 (2009) pp. 1497-1510

J.M. Torta, S. Marsal, J.J. Curto, L.R. Gaya-Piqué, Behaviour of the quiet-day geomagnetic variation at Livingston Island and variability of the Sq focus position in the South American-Antarctic Peninsula region. Earth Planets Space 62, 297-307 (2010)

R.H. Tyler, S. Maus, H. Lühr, Satellite observations of magnetic fields due to ocean tidal flow. Science 299, 239-241 (2003)

J. Uemoto, T. Maruyama, S. Saito, M. Ishii, R. Yoshimura, Relationships between pre-sunset electrojet strength, pre-reversal enhancement and equatorial spread-F onset. Ann. Geophys. 28, 449-454 (2010)

J. Untiedt, A model of the equatorial electrojet involving meridional currents. J. Geophys. Res. 72, 5799-5810 (1967)

T. Uozumi, K. Yumoto, K. Kitamura, S. Abe, Y. Kakinami, M. Shinohara, A. Yoshikawa, H. Kawano, T. Ueno, T. Tokunaga, D. McNamara, J.K. Ishituka, S.L.G. Dutra, B. Damtie, V. Doumbia, O. Obrou, A.B. Rabiu, I.A. Adimula, M. Othman, M. Fairos, R.E.S. Otadoy (MAGDAS Group), A new index to monitor temporal and long-term variations of the equatorial electrojet by MAGDAS/CPMN real-time data: EE-Index. Earth Planets Space 60, 785-790 (2008) 
D. van Sabben, Ionospheric current systems of the IGY-solar flare effects. J. Atmos. Terr. Phys. 22, 32-42 (1961)

D. van Sabben, Magnetospheric currents, associated with the N-S asymmetry of Sq. J. Atmos. Terr. Phys. 28, 965-981 (1966)

D. van Sabben, Solar flare effects and simultaneous magnetic daily variation, 1959-1961. J. Atmos. Terr. Phys. 30, 1641-1648 (1968)

D. van Sabben, The computation of magnetospheric currents, caused by dynamo action in the ionosphere. J. Atmos. Terr. Phys. 31, 469-474 (1969)

D. van Sabben, Solstitial Sq-currents through the magnetosphere. J. Atmos. Terr. Phys. 32, 1331-1336 (1970)

V.M. Vasyliūnas, The physical basis of ionospheric electrodynamics. Ann. Geophys. 30, 357-369 (2012)

J. Veldkamp, D. Van Sabben, On the current system of solar flare effects. J. Atmos. Terr. Phys. 18, 192-202 (1960)

E.H. Vestine, On the analysis of surface magnetic fields by integrals, part I. Terr. Magn. Atmos. Electr. 46(1), 27-41 (1941). doi:10.1029/TE046i001p00027

F. Vial, J.M. Forbes, Monthly simulations of the lunar semi-diurnal tide. J. Atmos. Terr. Phys. 56, 1591-1607 (1994)

G. Vichare, A. Bhaskar, D.S. Ramesh, Are the equatorial electrojet and the Sq coupled systems? Transfer entropy approach. Adv. Space Res. (2016). in press. doi:10.1016/j.asr.2016.01.020

C. Vineeth, T. Kumar Pant, R. Sridharan, Equatorial counter electrojets and polar stratospheric sudden warmings - a classical example of high latitude-low latitude coupling? Ann. Geophys. 27, 3147-3153 (2009)

C. Vineeth, N. Mridula, P. Muralikrishna, K.K. Kumar, T.K. Pant, First observational evidence for the connection between the meteoric activity and occurrence of equatorial counter electrojet. J. Atmos. Sol.-Terr. Phys. 147, 71-75 (2016)

H. Volland, J. Taubenheim, On the ionospheric current system of the geomagnetic solar flare effect (SFE). J. Atmos. Terr. Phys. 12, 258-265 (1958)

C.-U. Wagner, D. Möhlmann, K. Schäfer, V.M. Mishin, M.I. Matveev, Large-scale electric fields and currents and related geomagnetic variations in the quiet plasmasphere. Space Sci. Rev. 26(4), 391-446 (1980)

K. Weekes, M. Wilkes, Atmospheric oscillations and the resonance theory. Proc. R. Soc. A 192, 80-99 (1947)

D.R. Weimer, C.R. Clauer, M.J. Engebretson, T.L. Hansen, H. Gleisner, I. Mann, K. Yumoto, Statistical maps of geomagnetic perturbations as a function of the interplanetary magnetic field. J. Geophys. Res. 115, A10320 (2010). doi:10.1029/2010JA015540

M. Wiltberger, W. Wang, A.G. Burns, S.C. Solomon, J.G. Lyon, C.C. Goodrich, Initial results from the coupled magnetosphere ionosphere thermosphere model: magnetospheric and ionospheric responses. J. Atmos. Sol.-Terr. Phys. 66, 1411-1423 (2004)

D.E. Winch, Spherical harmonic analysis of geomagnetic tides, 1964-1965. Philos. Trans. R. Soc. A 303, $1-104$ (1981)

D.E. Winch, R.A. Cunningham, Lunar magnetic tides at Watherooo: seasonal, elliptic, evectional, variational, and nodal components. J. Geomagn. Geoelectr. 24, 381-414 (1972)

R.A. Wolf, Magnetospheric configuration, in Introduction to Space Physics, ed. by M.G. Kivelson, C.T. Russell (Cambridge Univ. Press, New York, 1995), pp. 288-329

R.F. Woodman, Vertical drift velocities and east-west electric fields at the magnetic equator. J. Geophys. Res. 75, 6249-6259 (1970)

D.L. Wu, P.B. Hays, W.R. Skinner, Observations of the 5-day wave in the mesosphere and lower thermosphere. Geophys. Res. Lett. 21, 2733-2736 (1994)

O.R. Wulf, A possible effect of atmospheric circulation in the daily variation of the Earth's magnetic field. Mon. Weather Rev. 91, 520-526 (1963)

W.-Y. Xu, Polar region Sq. Pure Appl. Geophys. 131, 371-393 (1989)

W.-Y. Xu, Effects of the magnetospheric currents on the Sq-field and a new magnetic index characterizing Sq dynamo current intensity. J. Geomagn. Geoelectr. 44, 449-458 (1992)

W.-Y. Xu, Y. Kamide, Decomposition of daily geomagnetic variations by using method of natural orthogonal component. J. Geophys. Res. 109, A05218 (2004). doi:10.1029/2003JA010216

T. Yabuzaki, T. Ogawa, Rocket measurement of Sq ionospheric currents over Kagoshima. Jpn. J. Geophys. Res. 79, 1999-2001 (1974)

A. Yacob, Seasonal parameters of the equatorial electro at different longitudinal zones. J. Atmos. Terr. Phys. 28, 581-597 (1966)

Y. Yamada, 2-day, 3-day, and 5-6-day oscillations of the geomagnetic field detected by principal component analysis. Earth Planets Space 54, 379-392 (2002)

Y. Yamada, Horizontal structure of the geomagnetic 2 day variation. J. Geophys. Res. 114, A12312 (2009). doi:10.1029/2009JA014307 
S. Yamashita, T. Iyemori, Seasonal and local time dependences of the interhemispheric field-aligned currents deduced from the Ørsted satellite and the ground geomagnetic observations. J. Geophys. Res. 107(A11), 1372 (2002). doi:10.1029/2002JA009414

Y. Yamazaki, Study on energy transfer of solar radiation and solar wind into equatorial ionosphere. $\mathrm{PhD}$ dissertation, Kyushu University, Fukuoka, Japan (2011)

Y. Yamazaki, Solar and lunar ionospheric electrodynamic effects during stratospheric sudden warmings. J. Atmos. Sol.-Terr. Phys. 119, 138-146 (2014)

Y. Yamazaki, M.J. Kosch, Geomagnetic lunar and solar daily variations during the last 100 years. J. Geophys. Res. Space Phys. 119, 6732-6744 (2014). doi:10.1002/2014JA020203

Y. Yamazaki, M.J. Kosch, The equatorial electrojet during geomagnetic storms and substorms. J. Geophys. Res. Space Phys. 120, 2276-2287 (2015). doi:10.1002/2014JA020773

Y. Yamazaki, A.D. Richmond, A theory of ionospheric response to upward-propagating tides: electrodynamic effects and tidal mixing effects. J. Geophys. Res. Space Phys. 118, 5891-5905 (2013). doi:10.1002/jgra.50487

Y. Yamazaki, K. Yumoto, A. Yoshikawa, S. Watari, H. Utada, Characteristics of counter-Sq SFE (SFE*) at the dip equator CPMN stations. J. Geophys. Res. 114, A05306 (2009). doi:10.1029/2009JA014124

Y. Yamazaki, K. Yumoto, T. Uozumi, S. Abe, M.G. Cardinal, D. McNamara, R. Marshall, B.M. Shevtsov, S.I. Solovyev, Reexamination of the Sq-EEJ relationship based on extended magnetometer networks in the east Asian region. J. Geophys. Res. 115, A09319 (2010). doi:10.1029/2010JA015339

Y. Yamazaki, K. Yumoto, M.G. Cardinal, B.J. Fraser, P. Hattori, Y. Kakinami, J.Y. Liu, K.J.W. Lynn, R. Marshall, D. McNamara, T. Nagatsuma, V.M. Nikiforov, R.E. Otadoy, M. Ruhimat, B.M. Shevtsov, K. Shiokawa, S. Abe, T. Uozumi, A. Yoshikawa, An empirical model of the quiet daily geomagnetic field variation. J. Geophys. Res. 116, A10312 (2011b). doi:10.1029/2011JA016487

Y. Yamazaki, K. Yumoto, T. Uozumi, M.G. Cardinal, Intensity variations of the equivalent Sq current system along the $210^{\circ}$ magnetic meridian. J. Geophys. Res. 116, A10308 (2011a). doi:10.1029/2011JA016632

Y. Yamazaki, A.D. Richmond, H. Liu, K. Yumoto, Y. Tanaka, Sq current system during stratospheric sudden warming events in 2006 and 2009. J. Geophys. Res. 117, A12313 (2012c). doi:10.1029/2012JA018116

Y. Yamazaki, A.D. Richmond, K. Yumoto, Stratospheric warmings and the geomagnetic lunar tide: 19582007. J. Geophys. Res. 117, A04301 (2012b). doi:10.1029/2012JA017514

Y. Yamazaki, K. Yumoto, D. McNamara, T. Hirooka, T. Uozumi, K. Kitamura, S. Abe, A. Ikeda, Ionospheric current system during sudden stratospheric warming events. J. Geophys. Res. 117, A03334 (2012a). doi:10.1029/2011JA017453

Y. Yamazaki, A.D. Richmond, A. Maute, H.-L. Liu, N. Pedatella, F. Sassi, On the day-to-day variation of the equatorial electrojet during quiet periods. J. Geophys. Res. Space Phys. 119, 6966-6980 (2014a). doi:10.1002/2014JA020243

Y. Yamazaki, A.D. Richmond, A. Maute, Q. Wu, D.A. Ortland, A. Yoshikawa, I.A. Adimula, B. Rabiu, M. Kunitake, T. Tsugawa, Ground magnetic effects of the equatorial electrojet simulated by the TIE-GCM driven by TIMED satellite data. J. Geophys. Res. Space Phys. 119, 3150-3161 (2014b). doi:10.1002/2013JA019487

Y. Yamazaki, M.J. Kosch, J.T. Emmert, Evidence for stratospheric sudden warming effects on the upper thermosphere derived from satellite orbital decay data during 1967-2013. Geophys. Res. Lett. 42, 61806188 (2015). doi:10.1002/2015GL065395

Y. Yamazaki, K. Häusler, J.A. Wild, Day-to-day variability of midlatitude ionospheric currents due to magnetospheric and lower atmospheric forcing. J. Geophys. Res. Space Phys. 121, 7067-7086 (2016). doi:10.1002/2016JA022817

M. Yasuhara, H. Maeda, Geomagnetic crochet of 15 November 1960. J. Atmos. Terr. Phys. 21, 289-293 (1961)

E. Yizengaw, M.B. Moldwin, A. Mebrahtu, B. Damtie, E. Zesta, C.E. Valladares, P. Doherty, Comparison of storm time equatorial ionospheric electrodynamics in the African and American sectors. J. Atmos. Sol.-Terr. Phys. 73, 156-163 (2011)

E. Yizengaw, E. Zesta, M.B. Moldwin, B. Damtie, A. Mebrahtu, C.E. Valladares, R.F. Pfaff, Longitudinal differences of ionospheric vertical density distribution and equatorial electrodynamics. J. Geophys. Res. 117, A07312 (2012). doi:10.1029/2011JA017454

E. Yizengaw, M.B. Moldwin, E. Zesta, C.M. Biouele, B. Damtie, A. Mebrahtu, B. Rabiu, C.F. Valladares, R. Stoneback, The longitudinal variability of equatorial electrojet and vertical drift velocity in the African and American sectors. Ann. Geophys. 32, 231-238 (2014)

X. Yue, W.S. Schreiner, J. Lei, C. Rocken, D.C. Hunt, Y.-H. Kuo, W. Wan, Global ionospheric response observed by COSMIC satellites during the January 2009 stratospheric sudden warming event. J. Geophys. Res. 115, A00G09 (2010). doi:10.1029/2010JA015466

K.Z. Zaka, A.T. Kobea, P. Assamoi, K.O. Obrou, V. Doumbia, K. Boka, B.J.-P. Adohi, N.M. Mene, Latitudinal profile of the ionospheric disturbance dynamo magnetic signature: comparison with the DP2 magnetic disturbance. Ann. Geophys. 27, 3523-3536 (2009) 
J.T. Zhang, J.M. Forbes, Lunar tidal winds between 80 and $110 \mathrm{~km}$ from UARS/HRDI wind measurements. J. Geophys. Res. Space Phys. 118, 5296-5304 (2013). doi:10.1002/jgra.50420

X. Zhang, J.M. Forbes, Lunar tide in the thermosphere and weakening of the northern polar vortex. Geophys. Res. Lett. 41, 8201-8207 (2014a). doi:10.1002/2014GL062103

J.T. Zhang, J.M. Forbes, Lunar tidal winds between 80 and $110 \mathrm{~km}$ from UARS/HRDI wind measurements. J. Geophys. Res. Space Phys. 118, 5296-5304 (2014b). doi:10.1002/jgra.50420

X. Zhang, J.M. Forbes, M.E. Hagan, Longitudinal variation of tides in the MLT region, 1: tides driven by tropospheric net radiative heating. J. Geophys. Res. 115, A06316 (2010a). doi:10.1029/2009JA014897

X. Zhang, J.M. Forbes, M.E. Hagan, Longitudinal variation of tides in the MLT region, 2: relative effects of solar radiative and latent heating. J. Geophys. Res. 115, A06317 (2010b). doi:10.1029/2009JA014898 